\title{
Quaderni Veneti
}

Nuova serie digitale

Vol. 6 - Num. 2 Dicembre 2017

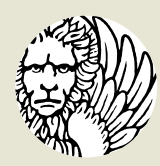

Edizioni Ca'Foscari 


\section{Quaderni Veneti}

[online] ISSN 1724-188X

Direttore

Tiziano Zanato

Edizioni Ca' Foscari - Digital Publishing

Università Ca' Foscari Venezia

Dorsoduro 3246, 30123 Venezia

http://edizionicafoscari.unive.it/it/edizioni/riviste/quaderni-veneti/ 


\title{
Quaderni Veneti \\ Rivista semestrale
}

\begin{abstract}
Direzione scientifica Tiziano Zanato (Università Ca’ Foscari Venezia, Italia)
Comitato scientifico Rossend Arqués Corominas (Universitat Autònoma de Barcelona, España) Ginetta Auzzas (Università degli Studi di Padova, Italia) Anna Maria Babbi (Università degli Studi diVerona, Italia) Eugenio Burgio (Università Ca' Foscari Venezia, Italia) Francesco Bruni (Università Ca' Foscari Venezia, Italia) Andrea Fabiano (Université ParisSorbonne, France) Ronnie Ferguson (University of St Andrews, UK) Franco Fido (Harvard University, Cambridge, MA, USA) John H. Hajek (The University of Melbourne, Australia) Giulio C. Lepschy (University College London, UK) Carla Marcato (Università degli Studi di Udine, Italia) Ivano Paccagnella (Università degli Studi di Padova, Italia) Manlio Pastore Stocchi (Università degli Studi di Padova, Italia) Gilberto Pizzamiglio (Università Ca' Foscari Venezia, Italia) Brian Richardson (University of Leeds, UK) Ricciarda Ricorda (Università Ca' Foscari Venezia, Italia) Guido Santato (Università degli Studi di Padova, Italia) Silvana Tamiozzo Goldmann (Università Ca' Foscari Venezia, Italia) Lorenzo Tomasin (Università di Losanna, Svizzera) Edward F. Tuttle (University of California, Los Angeles, CA, USA) Pier Mario Vescovo (Università Ca'Foscari Venezia, Italia) Alfredo Viggiano (Università degli Studi di Padova, Italia)
\end{abstract}

Comitato di lettura Tiziana Agostini (Venezia, Italia) Rossend Arqués Corominas (Universitat Autònoma de Barcelona, España) Ginetta Auzzas (Università degli Studi di Padova, Italia) Anna Maria Babbi (Università degli Studi di Verona, Italia) Cristina Benussi (Università degli Studi di Trieste, Italia) Michele Bordin (Università degli Studi di Ferrara, Italia) Francesco Bruni (Università Ca' Foscari Venezia, Italia) Eugenio Burgio (Università Ca' Foscari Venezia, Italia) Patrizia Cordin (Università degli Studi di Trento, Italia) Ilaria Crotti (Università Ca' Foscari Venezia, Italia) Andrea Fabiano (Université Paris-Sorbonne, France) Ronnie Ferguson (University of St Andrews, UK) Franco Fido (Harvard University, Cambridge, MA, USA) Serena Fornasiero (Università Ca' Foscari Venezia, Italia) Monica Giachino (Università Ca' Foscari Venezia, Italia) Giulio C. Lepschy (University College London, UK) John H. Hajek (The University of Melbourne, Australia) Emilio Lippi (Biblioteca Comunale, Treviso, Italia) Carla Marcato (Università degli Studi di Udine, Italia) Ivano Paccagnella (Università degli Studi di Padova, Italia) Manlio Pastore Stocchi (Università degli Studi di Padova, Italia) Paolo Pecorari (Università degli Studi di Udine, Italia) Gilberto Pizzamiglio (Università Ca' Foscari Venezia, Italia) Ricciarda Ricorda (Università Ca' Foscari Venezia, Italia) Brian Richardson (University of Leeds, UK) Michela Rusi (Università Ca' Foscari Venezia, Italia) Guido Santato (Università degli Studi di Padova, Italia) Silvana Tamiozzo Goldmann (Università Ca' Foscari Venezia, Italia) Lorenzo Tomasin (Università di Losanna, Svizzera) Edward F. Tuttle (University of California, Los Angeles, CA, USA) Pier Mario Vescovo (Università Ca' Foscari Venezia, Italia) Alfredo Viggiano (Università degli Studi di Padova, Italia) Tiziano Zanato (Università Ca' Foscari Venezia, Italia)

Direttore responsabile Lorenzo Tomasin (Università di Losanna, Svizzera)

\section{Direzione e redazione}

Università Ca' Foscari Venezia, Dipartimento di Studi Umanistici

Dorsoduro 3246, 30123 Venezia, Italia

Editore Edizioni Ca' Foscari - Digital Publishing | Dorsoduro 3246, 30123 Venezia, Italia | ecf@unive.it

Stampa Logo srl, via Marco Polo 8, 35010 Bogoricco (PD)

C 2017 Università Ca' Foscari Venezia

(C) 2017 Edizioni Ca' Foscari - Digital Publishing per la presente edizione

(c) Quest'opera è distribuita con Licenza Creative Commons Attribuzione 4.0 Internazionale

This work is licensed under a Creative Commons Attribution 4.0 International License

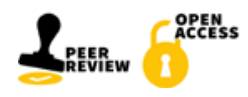


Vol. 6 - Num. 2 - Dicembre 2017

\section{Sommario}

GIOVAN BATTISTA RAMUSIO. NUOVE INDAGINI FILOLOGICHE

E LINGUISTICHE

\section{Premessa}

Francesco Crifò, Anna Rinaldin

Tradizioni attive e ipertesti

Ramusio 'editore' del Milione

Samuela Simion

"... io essendo giovanetto n'ho udito molte fiate dire...»"

Alcune riflessioni sulle fonti dei Viaggi di messer Marco Polo di Giovanni Battista Ramusio

Alvise Andreose

\section{Sulla fonte $\mathbf{Z}$ del Milione di Ramusio}

L'enigma di Quinsai

Giuseppe Mascherpa

Filologia dei testi volgari e ambienti digitali

Qualche annotazione a margine del Ramusio digitale

Eugenio Burgio

La variazione lessicale nel Milione

Interferenza linguistica e costanti interpretative

Irene Reginato

L'ambiguità del teonimo poliano Natigay-Načigay

Elisabetta Ragagnin 


\section{«Reverendo signore mio» \\ Note sulla lingua di Ramusio dalla corrispondenza con Pietro Bembo}

Fabio Romanini

«Per convenienti rispetti»

Osservazioni sulla presa di parola di G.B. Ramusio

Toni Veneri

Bibliografia generale

Certificazione scientifica delle Opere pubblicate da Edizioni Ca' Foscari - Digital Publishing: tutti i saggi pubblicati hanno ottenuto il parere favorevole da parte di valutatori esperti della materia, attraverso un processo di revisione anonima sotto la responsabilità del Comitato scientifico della collana. La valutazione è stata condotta in aderenza ai criteri scientifici ed editoriali di Edizioni Ca' Foscari.

Scientific certification of the works published by Edizioni Ca' Foscari - Digital Publishing: all essays published in this volume have received a favourable opinion by subject-matter experts, through an anonymous peer review process under the responsibility of the Scientific Committee of the series. The evaluations were conducted in adherence to the scientific and editorial criteria established by Edizioni Ca' Foscari.

Qualunque parte di questa pubblicazione può essere riprodotta, memorizzata in un sistema di recupero dati o trasmessa in qualsiasi forma o con qualsiasi mezzo, elettronico o meccanico, senza autorizzazione, a condizione che se ne citi la fonte.

Any part of this publication may be reproduced, stored in a retrieval system, or transmitted in any form or by any means without permission provided that the source is fully credited. 


\title{
Giovan Battista Ramusio. Nuove indagini filologiche
} e linguistiche

\author{
a cura \\ di Francesco Crifò e Anna Rinaldin
}



Vol. 6 - Num. 2 - Dicembre 2017

\title{
Premessa
}

\author{
Francesco Crifò
}

(Universität des Saarlandes, Saarbrücken, Deutschland)

Anna Rinaldin

(Università di Fiume, Croazia)

Il 23 giugno del 2017 l'Università del Saarland ha ospitato un incontro di otto fra le maggiori autorità sulla figura e l'opera di Giovan Battista Ramusio (1485-1557), noto soprattutto come editore dell'epocale raccolta in tre volumi Delle navigationi et viaggi. L'eccentrica ubicazione dell'incontro si giustifica con il grande interesse che l'opera riveste per gli studi sulla storia del lessico italiano. Fuori d'Italia, questi hanno trovato in Saarbrücken una sede di prima importanza da quando Max Pfister vi stabilì nel 1974 il suo laboratorio, codiretto dal 2001 da Wolfgang Schweickard. L'analisi delle caratteristiche filologiche delle fonti ha sempre rivestito un ruolo centrale nella loro scuola.

È questo il trait d'union con l'innovativa edizione critica digitale del testo ramusiano Dei viaggi di Messer Marco Polo gentiluomo veneziano, primo tassello dell'ambizioso progetto che vedrà la messa in rete di tutte le redazioni del testo poliano.

I primi quattro contributi qui raccolti si devono ai responsabili e ad alcuni dei principali collaboratori del progetto. Samuela Simion, Alvise Andreose e Giuseppe Mascherpa presentano nei rispettivi contributi altrettante dimostrazioni di come i lavori di edizione digitale abbiano portato con sé nuovi dati e considerazioni innovative sulla complessa tradizione del Marco Polo ramusiano. Eugenio Burgio prende invece spunto dall'edizione per contribuire al dibattito internazionale sulle nuove frontiere della filologia editoriale. Irene Reginato ed Elisabetta Ragagnin esplorano a titolo esemplare alcune delle molte stimolanti questioni etimologiche che il Polo di Ramusio pone alla comunità scientifica. Infine Fabio Romanini e Toni Veneri rivolgono l'attenzione alla figura storica, all'opera e allo stile di Ramusio, rispettivamente in termini (prevalentemente) storico-linguistici e letterari-epistemologici.

Rivolgiamo con piacere un ringraziamento particolare a Wolfgang Schweickard, che è stato prodigo di importanti stimoli per la discussione non meno che per la pianificazione del convegno, a Candida Andreas-Cuva, efficientissima responsabile dell'organizzazione, e a tutti i partecipanti 
nonché autori della presente raccolta. Pur non avendo avuto modo di partecipare alla giornata di studi, Max Pfister l'ha incoraggiata mostrando ancora una volta l'interesse partecipe che ha sempre riservato anche alle iniziative degli studiosi più giovani: non è questa l'ultima delle molte ragioni per cui la raccolta che qui presentiamo è dedicata alla sua memoria. 


\title{
Tradizioni attive e ipertesti Ramusio 'editore' del Milione
}

\author{
Samuela Simion \\ (Università Ca’ Foscari Venezia, Italia)
}

\begin{abstract}
The contribution focuses on a scholarly digital project presented in 2015 by Eugenio Burgio, Marina Buzzoni and Antonella Ghersetti, Dei Viaggi di Messer Marco Polo, the Italian version of the Devisement dou monde/Milione by Giovanni Battista Ramusio (1559), freely available at Edizioni Ca' Foscari - Digital Publishing website. The paper describes the intellectual reasons that have bolstered the project, in the new context provided by the so-called digital philology, and shows a demo that illustrates its operational mechanisms. Finally, it presents some outcomes of the project: (a) a new hypothesis of stemma codicum, which tries to explain the ways of transmission and the relationships between the two traditional branches $\alpha$ and $\beta$; (b) a new scholarly digital project: the critical edition of Marco Polo's book, started out as the logical development of the Ramusio digitale.
\end{abstract}

Sommario 1 Introduzione. - 2 Il Ramusio digitale. - 3 La tradizione del Devisement dou monde/ Milione. - 4 Perché un'edizione critica digitale. - 5 Limiti della mise en page tradizionale. - 6 Una nuova ipotesi stemmatica per il Devisement dou monde/Milione. - 7 Verso un «Marco Polo integrale».

Keywords Marco Polo. Giovanni Battista Ramusio. Digital scholarly projects. Textual studies. Romance philology.

\section{Introduzione}

Negli ultimi anni i progetti che sfruttano il supporto digitale si sono moltiplicati, concretizzandosi in prodotti eterogenei per obiettivi e per metodo; ${ }^{1}$ è significativo che, accanto alle edizioni vere e proprie, ${ }^{2}$ circolino ormai numerosi manuali, di natura operativa o teorico-metodologica, segno del

1 Per un quadro aggiornato dei progetti in corso, indicativo del fermento che anima il settore, cf. Driscoll, Pierazzo 2016a. Oltre alla manualistica, alle monografie, a una massa considerevole di articoli, le digital humanities (e la digital philology) si stanno diffondendo anche grazie a riviste e piattaforme nate appositamente: si possono citare «Digital Philology», «Digital Humanities Quarterly», «Scholarly Editing», e il sito tedesco RIDE (collettore di recensioni di edizioni digitali); su iniziative come il Network for Digital Methods in the Arts and Humanities (NeDiMAH), cf., di nuovo, Driscoll, Pierazzo 2016a, 1.

2 Il censimento online curato da Patrick Sahle (2017) ne conta oltre 400. La classificazione tipologica proposta dallo studioso (per titolo; area tematica; materiale; lingua; epoca) rende immediatamente evidenti alcune linee di tendenza: le edizioni digitali di opere moderne sono 
successo della filologia digitale e conferma che essa sta entrando in una fase di codificazione e standardizzazione. Standardizzazione che appare però un obiettivo di non immediato conseguimento, visto che la lussureggiante varietà di oggetti digitali si lascia difficilmente fissare in norme univoche: paradossalmente anche concetti fondamentali - come la definizione stessa di edizione digitale - presentano margini di oscillazione e di ambiguità non risolti, malgrado i numerosi sforzi per approdare a una nomenclatura condivisa. ${ }^{3}$

In generale, i primi bilanci di vent'anni di digital philology sembrano concordi, in Italia e all'estero, e indipendentemente dall'approccio metodologico di ciascun editore, nell'individuare due filoni di attrazione principali: (a) nel primo caso l'efficacia del digitale nel rappresentare la varianza testuale ha sancito la fortuna delle edizioni genetiche, grazie anche alla mobilità che accomuna medium digitale e il processo di correzioni oggetto della critique génétique (cf. Italia 2016b, 247-8); (b) un secondo fuoco d'interesse privilegia invece il 'documento' singolo, tangibile, rispetto al testo ricostruito: ${ }^{4}$ molti progetti prevedono la digitalizzazione dei manoscritti, corredata o meno dalla loro trascrizione, e la possibilità di affiancarli in sinossi. A volte l'edizione documentaria è una necessità inderogabile, quando si pubblicano documenti d'archivio o quando si lavora in una

le più numerose; in genere si pubblicano di più singole opere o manoscritti, meno documenti come i diari o le iscrizioni; forte e non inattesa è la preponderanza dell'inglese.

3 A questo proposito cf. almeno Price 2009, Sahle 2016 e Robinson 2016. Come sostiene Andrews $(2013,62)$, «consensus is indeed lacking on what exactly a digital critical edition should be. As long as there is no agreement on the end result of digital philology, there can be none on its methods; as long as there is no consensus on method, there will not be widely applicable computational tools available to help produce digital critical texts». Più in generale, a rimanere in parte sfuggenti sono la direzione e lo statuto stesso della filologia digitale: «What seems even more compelling, however, is to understand what digital scholarly editing actually is: is it a new discipline or a new methodology? Are we simply putting 'old wine in new bottles', or are we doing something which has never been done [...] before?» (Driscoll, Pierazzo 2016b, 3).

4 Come osserva Leonardi $(2007,67)$, «nella maggior parte delle edizioni digitali, anche quelle che non si limitano a dar conto di un solo testimone, l'attenzione è focalizzata molto più sulla riproduzione, diciamo pure sull'edizione, di ciascun singolo manoscritto, e sulla corrispondenza di questa alla pagina visualizzata del codice, o al massimo sulla possibilità di affiancare i diversi individui nelle finestre dello schermo, che non sulle potenzialità di un confronto approfondito sul piano testuale tra le diverse unità testimoniali». Cf. anche Robinson (2016, 191): «Many of these editions are what one might call digital facsimile transcripts, focusing on a single manuscript and recording its text in precise form, page by page, line by line and character by character. These editions - and they most certainly are editions, in the basic sense that an editor is scrutinizing every mark on the page - characteristically focus on two elements: the exact disposition of the text on each page and on the writing process. The first of these corresponds to 'diplomatic' or 'facsimile print' editions, the second to 'genetic editions'». 
prospettiva paleografica o codicologica; ${ }^{5}$ spesso però la visione teorica di fondo risente della fascinazione esercitata dalla maggiore 'oggettività' del documento singolo, e si colloca nell'onda lunga della New Philology, proiettando anche sulla 'nuova' filologia digitale l'ombra della mai tramontata opposizione «fra conservazione e ricostruzione, tra la sincronia del singolo manoscritto e la diacronia genealogica dello stemma, tra la verità del copista e la verità dell'autore, insomma tra gli eponimi Bédier e Lachmann». ${ }^{6}$ Uno degli aspetti più vistosi della 'rivoluzione digitale' è la creazione tumultuosa di archivi e biblioteche virtuali: l'offerta di materiali non è però accompagnata da adeguati filtri interpretativi, con l'effetto paradossale di un generale sottoutilizzo delle potenzialità del mezzo (che ha tra i suoi risvolti anche l'esiguità complessiva di edizioni critiche). ${ }^{7}$

\section{Il Ramusio digitale}

In questo quadro di necessità molto semplificato, ${ }^{8}$ il Ramusio digitale si colloca in una sorta di 'terza via', e offre, nelle nostre intenzioni, una possibilità concreta di superamento delle vecchie antinomie, e uno strumento informativo aperto a più discipline. ${ }^{9}$

Nato inizialmente con la volontà di divulgare i risultati di un seminario su Ramusio svolto a Venezia nel 2010 (per gli atti cf. Burgio 2011), il progetto si è progressivamente ampliato, restando incardinato su una prospettiva pragmatica attenta piuttosto a mettere a frutto le peculiarità

5 «Un'edizione basata su un testimone unico, infatti, può essere diplomatica, semidiplomatica, o critica, a seconda del tipo di testo (un atto notarile, o una lettera, che è documento, verranno pubblicati in edizione diplomatica e non critica), della condizione in cui è giunto a noi, e del tipo di intervento che necessita» (Italia 2016b, 248).

6 Cf. Leonardi 2007, 65. Uno dei versanti in cui la digital philology sta elaborando strumenti molto sofisticati è, non a caso, la riproduzione diplomatica dei documenti; si tratta, per Robinson $(2016,184-7)$, di una delle frontiere in cui il supporto digitale potrà dare i frutti più rivoluzionari.

7 Così Robinson $(2016,182)$ : «We now have thousands of manuscripts and millions of books online. And what are people doing with all this? The language used to describe these collections is revealing: in almost no case, are these abundances of materials described as 'editions'. They are 'archives', 'thematic research collections', perhaps 'arsenals', but not editions».

8 Per una messa a fuoco più precisa rinvio all'intervento di Eugenio Burgio in questo volume.

9 Come sostengono Buzzoni, Burgio (2014, 178), il Ramusio digitale si colloca infatti in un'ideale «third way», in quanto offre un «hyper-textual environment and a hyper-textual way of using the digital edition [...] based not on a static but on an interactive model. The main principles followed are text mobility, on the one hand, and flexibility of text representations, on the other»; in questo modo è possibile «to convey the notion of diasystem as defined by Segre, as well as to make the reader appreciate how the text develops over time in the Continian fashion». 
del vettore (in termini di quantità di dati, duttilità, interoperabilità, interattività, multimedialità, visualizzazione simultanea dei dati e facilità di interrogazione),$^{10}$ che a inserirsi nel dibattito teorico (a rigore, per un filologo digitale 'ortodosso' la nostra edizione non è un'edizione critica digitale, perché si affida largamente al lavoro manuale anziché a metodi automatizzati, ad esempio in fase di trascrizione e collazione).

La natura stessa dell'oggetto del nostro lavoro ci ha posto di fronte a una declinazione particolare del metodo ricostruttivo: avevamo già in partenza un testo ricostruito, il Milione ramusiano, che risulta dal montaggio di più versioni poliane. Si trattava di scomporre questo testo per comprendere quali e quanti fossero i testimoni-fonte impiegati da Ramusio e quale fosse il loro apporto, in modo da poter poi gerarchizzare le informazioni in una rappresentazione adeguata.

Perché Ramusio? In prima battuta perché il Marco Polo di Ramusio (a cui mi riferirò anche come R) costituisce, come intuì negli anni '20 Luigi Foscolo Benedetto, la via maestra per razionalizzare la difficile tradizione del Milione: i codici usati da Ramusio sono spesso più ricchi di informazioni rispetto al testimone siglato $\mathrm{F}$ (il codice fr. 1116 della Bibliothèque nationale de France), di norma assunto come testo base in virtù della sua facies linguistica franco-italiana, ritenuta originale (e conservata, oltre che in $\mathrm{F}$, in un frammento affine, $f) .{ }^{11}$ Tuttavia, l'interesse degli studiosi nei confronti delle novità contenute nel Milione ramusiano venne a lungo temperato dall'idea che si trattasse di interpolazioni, equivoco alimentato anche dal fatto che Ramusio indicò solo genericamente i manoscritti a sua disposizione.

Il quadro tracciato da Benedetto, che resta ancora un riferimento negli studi poliani, può essere così sintetizzato: Benedetto individuò le fonti delle aggiunte ramusiane nelle redazioni siglate $\mathrm{Z}, \mathrm{P}, \mathrm{L}$ (latine), VB, V (veneziane), e comprese che il problema andava spostato da Ramusio ai codici del Milione che le contenevano. In questo modo riuscì a raggruppare la tradizione in due famiglie, A e B, indicando nella seconda la testimone di una 'fase anteriore a $\mathrm{F}^{\prime}$ ', cioè una fase più vicina all'originale perduto, più ricca nei contenuti, di sicura paternità poliana. La sua ipotesi non prese

10 Cf. Buzzoni (2016, 59-60), che insiste poi (62) su un aspetto per noi centrale: «A major advantage of a digital edition is its potential to provide a model capable of embodying the edition-in-time ('edizione-nel-tempo') as a result of text-in-time ('testo-nel-tempo') postulated by Gianfranco Contini».

11 La cura particolare dedicata al Milione si riflette tra l'altro nell'allestimento del testo, nella sua presentazione introdotta da tre paratesti, nella levigatura linguistica, coerentemente con il ruolo culturale e politico che Marco Polo e il suo viaggio assumono nell'economia della silloge ramusiana, cerniera tra vecchia e nuova geografia e strumento di rivendicazione della centralità di Venezia nel quadro di riassestamento degli equilibri geo-politici in corso nel Cinquecento; cf. Simion (in corso di stampa (a)), che ripercorre l'Introduzione di Simion, Burgio 2015. 
la forma di uno stemma codicum, perché l'immagine dell'albero sembrava inadatta a Benedetto a rappresentare una storia genealogica meglio definibile in termini di «broussaille, sous-bois enchevêtré». ${ }^{12}$

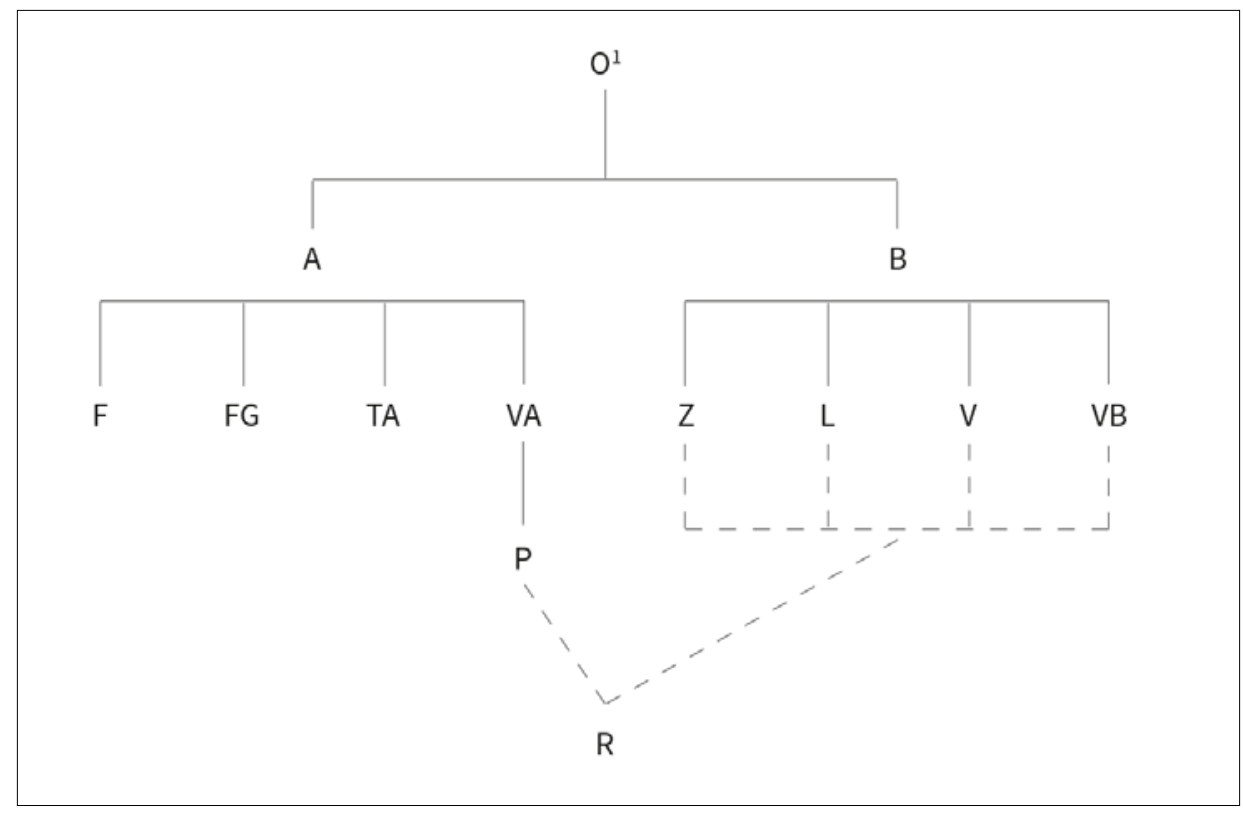

Figura 1. La tradizione manoscritta del Milione delineata da Luigi Foscolo Benedetto (1928)

Benedetto osservò inoltre che $\mathrm{R}$ e $\mathrm{Z}$ condividono molti passi privi di attestazione nella 'vulgata', ma che a sua volta $\mathrm{R}$ ne contiene altri assenti in $\mathrm{Z}$ : dal che è possibile inferire che lo $\mathrm{Z}$ usato da Ramusio, il perduto 'codice Ghisi' (dal nome del possessore citato nella lettera dedicatoria), doveva essere più completo dell'unico manoscritto superstite, il toledano Zelada 49.20, che appare molto abbreviato nella prima parte, e manca in totale di circa 60 capitoli rispetto a F.

\section{La tradizione del Devisement dou monde/Milione}

Questi brevi cenni esemplificano già alcune delle difficoltà poste dalla tradizione del Milione. Ne richiamerò per sommi capi i punti più importanti

12 «Ce n'est certes pas par l'image d'un arbre qu'on peut résumer l'histoire généalogique d'un texte tel que celui de Marco. C'est plutôt de broussaille qu'il faut parler, de sous-bois enchevêtré» (Simion 2016, 171). 


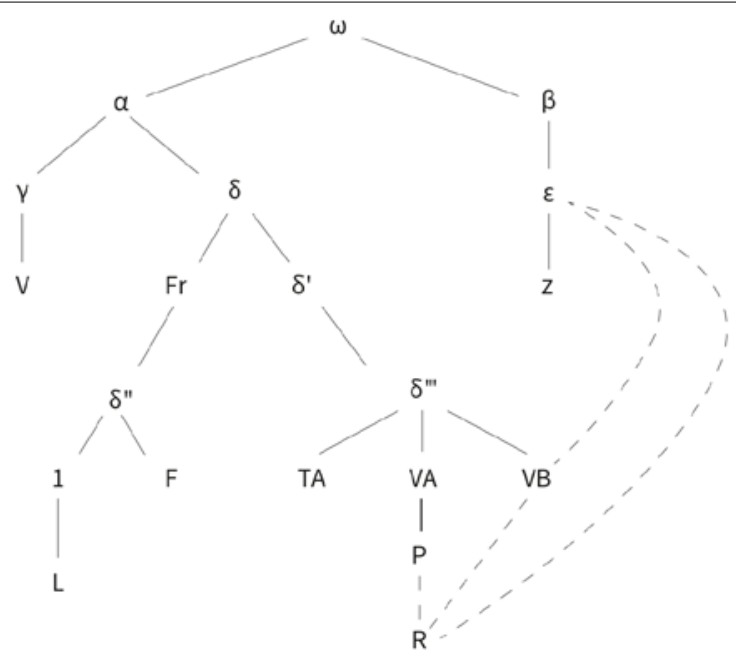

Figura 2. Stemma codicum del Devisement dou monde secondo la proposta di Burgio, Eusebi 2008

per il nostro ragionamento, utilizzando la proposta stemmatica di Burgio, Eusebi (2008), ma anticipando che ne stiamo modificando i piani alti.

L'archetipo $\omega$ si divide in un albero bipartito, in cui al ramo $\beta$ (definibile grazie a $\mathrm{Z}$ e a R), si oppone il ramo $\alpha$, formato dai sottogruppi $\delta$ (F, Fr, L, TA, VB, VA, P) e $\gamma$ (coincidente con V). ${ }^{13}$ Come si vede, lo stemma Burgio, Eusebi (2008) presenta alcune importanti differenze rispetto allo schema di Benedetto: di fatto la «famiglia B» si dissolve, perché V, L, VB vengono

13 Le sigle delle redazioni: $\mathrm{F}$ = redazione franco-italiana del XIV sec., tràdita dal ms. fr. 1116 della Bibliothèque nationale de France (Eusebi [2010] 2018) e dal frammento scoperto da Chiara Concina, siglato f, smembrato in due parti, ora in collezioni private (Concina 2007; Ménard 2012; un'analisi di $\mathrm{f}$ in rapporto a $\mathrm{F}$ in Andreose, Concina 2016); $\mathrm{Fr}=$ redazione francese tràdita da 18 mss., i più antichi dei quali di inizio Trecento (Ménard 2001-9); K = redazione «catalana», tràdita da tre testimoni, XIV sec. (Reginato 2015-16); L = compendio latino trecentesco tràdito da 6 testimoni (Burgio, in corso di stampa); $\mathrm{P}=$ traduzione in latino condotta su un esemplare VA da Francesco Pipino O.P., entro il primo quarto del XIV sec.; tràdito da una sessantina di testimoni (trascrizione interpretativa di Simion 2015a; Prášek 1902); TA = redazione toscana primo-trecentesca, tràdita da 5 manoscritti (Bertolucci Pizzorusso 1975); TB = redazione toscana tardo-trecentesca, tràdita da 7 codici (Amatucci 1982-3); $\mathrm{V}=$ versione veneziana tràdita dal ms. Staatsbibliothek, Hamilton 424, tardo quattrocentesco (Simion 2008-09); VA = redazione veneto-emiliana, tràdita da cinque testimoni, il più antico dei quali di inizio Trecento (Barbieri, Andreose 1999); VB = rimaneggiamento veneziano, tràdito da due codici e un frammento, tutti del XV secolo (Gennari 2008-09); Z = versione latina tràdita dal ms. Zelada 49.20 dell'Archivo y Biblioteca Capitulares di Toledo, della metà del XV sec. (Barbieri 1998). 
spostati in $\alpha$, L viene disgiunto da $\mathrm{V}$, e $\beta$ è così rappresentato dal solo $\mathrm{Z}$. Per quanto riguarda $\mathrm{R}$, di cui è accentuato l'aspetto di collettore di varianti, l'analisi propedeutica all'allestimento del database ha dimostrato che gli apporti davvero sicuri provengono da $\mathrm{P}, \mathrm{VB}$ e $\mathrm{Z}$, mentre più sfocati e sporadici appaiono gli accordi con L e V (cf. Burgio 2011).

Siamo di fronte a una tradizione attiva: gli oltre 140 codici superstiti fanno capo a famiglie che rappresentano altrettante redazioni dell'opera, con un polimorfismo di contenuti (rimaneggiamenti, compendi, riscritture) e di lingua (di fatto, con l'eccezione di F, disponiamo unicamente di traduzioni, nelle principali lingue volgari e in latino). Il caso forse estremo, ancora poco studiato, è rappresentato dalla versione tràdita dal cosiddetto 'codice Vaglienti' (pubblicato da Formisano 2006, 57-102): volgarizzamento toscano di un testo latino (LA), tradotto da un testo toscano (TB), esemplato a partire da un modello lombardo-emiliano (VA) che risale a un subarchetipo franco-italiano. Oltre che con le normali varianti, si fanno insomma i conti con adattamenti plurimi e con catene complesse di interferenze linguistiche. A monte di ciascuna redazione ci sarebbe, secondo l'ipotesi di Benedetto da noi accolta, una serie di copie perdute in franco-italiano, affini a $F$, la cui esistenza è dimostrabile attraverso le tracce, soprattutto lessicali, vere e proprie buttes témoins ${ }^{14}$ che il franco-italiano di partenza ha lasciato nelle traduzioni d'arrivo: l'intervento di Irene Reginato in questo volume offre un campione di questi livelli di interferenza, e dimostra una volta di più come l'analisi del lessico si collochi in un rapporto vitale con la ricostruzione genealogica, permettendo di rintracciare la presenza di costanti e la serialità di fenomeni, e di delineare così una sorta di 'tipologia dell'innovazione' (cf. Leonardi 2007, 70-1).

In aggiunta al tasso di varianza complessivo va inoltre tenuto conto di un fattore che si tende a dare per scontato, ma che rischia di travisare la comprensione del processo di trasmissione testuale: ai rami alti dello stemma, o nelle sue articolazioni fondamentali, la nostra riflessione si basa su redazioni monotestimoniali ( $\mathrm{Z}$ per i contenuti, $\mathrm{F}$ per la lingua, $\mathrm{V}$ per l'individuazione di una fase intermedia tra $\alpha$ e $\beta$ ), il che porta inevitabilmente, ma pericolosamente, ad assolutizzarne la testimonianza, e a sopravvalutarne l'individualità. Il rischio era già chiaro a Terracini (1933, 393 nota 1 ), che a proposito dello $\mathrm{Z}$ toledano ammoniva:

In linea generale occorre osservare che la stessa bontà di Z fornisce [...] un'arma un poco pericolosa: da una parte l'ottima opinione che noi abbiamo del ms ci rende più schizzinosi, d'altro lato siamo involontaria-

14 Riprendo la metafora geologica da Ménard $(2005,422)$, che la usa a proposito degli italianismi diffusi nella redazione francese Fr («Des vestiges subsistent, à la façon des buttes témoins dans le paysage. Ces éléments sont révélateurs de la strate ancienne, recouverte par la mise en bon français»). 
mente indotti, e sovente non senza ragione, a ritenere sicuro un passo, appunto per il fatto che ce lo dà $Z ;^{15}$

mentre per F il discorso vale soprattutto a livello linguistico:

Stabilire cosa si debba ascrivere al copista - o, meglio, ai copisti - equivale a determinare che cosa fosse la lingua della redazione originaria; è cioè un problema insolubile, perché non si può escludere che questa fosse già profondamente ibrida e disuguale. (Benedetto 1929, 40) ${ }^{16}$

Nel caso di V, infine, il problema principale è rappresentato dall'eccentricità del testo tràdito dal berlinese Hamilton 424, la cui fisionomia è il risultato di un accumulo di incidenti di copia, cui si somma la tendenza dell'ultimo copista ad attuare interventi sensum de sensu, vòlti ad 'aggiustare' il senso delle pericopi poco chiare; in questo modo vengono però spesso occultate le linee di derivazione ed offuscati i passi difficiliores, in cui $\alpha$ e $\beta$ si contrappongono.

Malgrado questi ostacoli è possibile riconoscere anche alcune grandezze costanti (errori comuni, almeno in $\delta$, e contenuti che si ripetono con la stessa disposizione nelle varie redazioni), ed è su questi 'tratti pertinenti' che si giustifica la nostra proposta di classificazione.

\section{Perché un'edizione critica digitale}

Preso atto dell'interesse del Milione di Ramusio sia come 'oggetto' individuo che come accessus al Milione, si trattava quindi di tradurre in un'edizione i materiali risultanti dalla collazione delle varie redazioni con $\mathrm{R}$, tenendo insieme la rappresentazione delle procedure editoriali di Ramusio, la trascrizione e collazione delle fonti e di F, e un commento aggiornato, con l'interrogazione dei dati. La forma editoriale cartacea risultava poco efficace rispetto alla dimensione comparatistica che ci interessava, come appariva evidente dalle edizioni «integrali» del Milione in circolazione

15 Burgio e Mascherpa $(2007,123)$ estendono la cautela anche al piano linguistico: «il manoscritto Z, ascrivibile alla seconda metà del sec. XV, è copia di una copia, ragion per cui non è facile stabilire sicuramente in quale misura i fenomeni riconducibili alla grammatica del volgare siano da attribuire al 'sistema' dell'ultimo copista e non, piuttosto, ai precedenti intermediari, se non addirittura all'autore della versione, redatta, come si evince dalla sua tradizione indiretta, entro il primo terzo del sec. XIV». Ben più radicale il giudizio di Ménard (2001-09, 1: 16): «le manuscrit $Z$ ne mérite pas la confiance aveugle qui lui a été portée».

16 Cf. anche Bertolucci Pizzorusso $(1984,799)$. I recenti sondaggi sulla lingua di F (e f) e sul suo ambiente di produzione (l'atelier pisano-genovese) condotti da Andreose (2015b, 2015c, 2015d) e Andreose, Concina (2016) permettono inoltre di illuminare meglio la complessità della stratigrafia linguistica e di tentarne un'interpretazione. 
(Benedetto 1928; Moule, Pelliot 1938). L'inadeguatezza della mise en page tradizionale può essere esemplificata prendendo in esame qualsiasi luogo testuale che presenti delle difficoltà; un buon punto di partenza può essere, ad esempio, il passo dedicato al traino delle navi lungo il fiume Quian, l'odierno Fiume Azzurro, in cinese Jiang (R II 63). Poiché il testo di riferimento è la versione franco-italiana $\mathrm{F}$, partiremo proprio dalla lezione di F CXLVI.

Il capitolo è dedicato alla città di Singui: si tratta, secondo Pelliot (195973, 832-4 nota 338), «dell'attuale Yizheng 仪征, nel Jiangsu, a circa $30 \mathrm{~km}$ a Sud-Ovest di Yangzhou, chiamata in epoca mongola Zhenzhou»; Pelliot ritiene inoltre che la digressione sulle navi sia «motivata dall'importanza della tassa sul sale nella città» (Simion, Burgio 2015, s.v. «Singiu»).

Con un'attenzione alle imbarcazioni documentate anche altrove nel testo (cf. Ménard 2008, 2010), Marco Polo loda il numero di navi che sosta sul fiume, indicativo della vivacità commerciale della città; si tratta di imbarcazioni con una coperta, un albero e una vela, di grande portata. Il capitolo si conclude con la descrizione del traino delle barche da terraferma per mezzo di spilorce di bambù; viene fornito infine qualche cenno alla fabbricazione di queste particolari funi, la cui resistenza è elogiata rispetto alla più tradizionale canapa:

Or sachiés qe tutes les nes ne ont sarce de caneve, for que il en ont bien forni les arbres e les voiles. Mes je vos di qu'ele ont le pelorce de canne, con le quele

FCXLVI 9 se tirent les nes sor por cest flum. Et entendés qe cest sunt de les cannes groses et longes, qe je voç ai dit en ereres, qe bien sunt longes .XV. pas: il le fendent e ligent le une con l'autre et le font long bien .iiic. pas, et est plus fort qe ne seroit de chanave.

Pur senza significative escursioni sul piano del senso, il passo non si presenta in maniera compatta nella tradizione: ${ }^{17}$

Et vous di que le corde dont il se font tirer, qui est bien longue .CCC. pas, n'est d'autre chose que de cane si faites: il ont canes bien longues .XV. pas,

$\operatorname{Fr} 14643-50$ si comme je vous ay autre fois dit en ce livre, et premierement il prennent ces canes et les fendent du lonc par mi et lient l'une a l'autre et en font tant longues leurs cordes comme il veullent, et les font moult fors.

17 Cito il testo di K secondo la lezione del suo rappresentante catalano (Kc); il ramo K (che Benedetto 1928, CCI-IX collocava tra gli «Echi e frammenti vari» del Milione) è però formato anche da due versioni di un archetipo catalano: l'aragonese Ka e la francese Kf. 
E no han negunes cordes; e con tiren lurs naus contra la corrent, han de molt

Kc 638 grosses cayes que han en lurs terres, e fenen-les e liguen-la una contra l'autra, e fan-ne tant longua corda que ha bé CCC paces de lonch; e ab aquestes cayes aytals han de lonch bé XX paces e ha bé un palm de gros (la sua gruxa qui va tro al buyt).

Et sunt omnes, hee naves, cohoperte, ferentes arborem un`am` solum. Trahunt autem has naves per flumen non cum funibus; sed cannas magnas (de quibus supradictum est) que sunt longe passus .XV. findunt, caputque capiti ligant sicque eas .CCC. passus elongant, cum quibus naves trahunt; sunt enim funibus fortiores.

Non usano corde di canevo se non per l'arbore della nave, per la vela, ma hanno canne lunghe da quindeci passa, come habbiamo detto di sopra, le quali sfendono da un capo all'altro in molti pezzi sottili, et poi le piegano

R II $637-8$ insieme et fanno di quelle tortizze lunghe trecento passa, non meno forti che le tortizze di canevo, tanto sono con gran diligenza fatte. Con queste in luogo di alzana si tirano su per il fiume le navi, et ciascuna ha dieci o dodici cavalli per far questo effetto di tirarle all'incontro dell'acqua, et ancho a seconda.

Le nave son coverte e ànno un àlbore, ma sono di grande portare, che ben portano da. iiij $^{m}$. cantari insino in . $\mathrm{xij}^{\mathrm{m}}$. cantari. Tutte le navi ànno sarta di

TA 1438 canave, cioè legami per legare le navi e spers tiralle su per questo fiume. Le piccole sono di canne grosse e grandi, com'io v'ò detto di sopra; elli legano l'una all'altra, e fannole lunghe bene .iijc. passi e fendole; e sono più forti che di canave.

Sapié che queste nave non àno sartie de chanevo, ma sollamente i albori

V $727-8$ fornidi de velle; et àno resti de erba chon li qualli i tirano le velle, et sono molti grossi e longi. Or queste nave sono longe diexe passa, et zaschaduna de queste nave àno dodexe chavali i qualli tirano quelle per lo fiume.

Le nave grande de questa provinzia àno una choverta e uno alboro e non àno sartie de chanevo, se non solamente per i arbori e per le velle, e i àno pilore 0 ,

VA CXIII 7-10 per dir chiaro, alzane de chane, chon le qual se tira le nave su per el fiume. $\mathrm{E}$ sono fate delle chane grosse che ò dito de sopra, le qual son longe ben sedexe passa. I lle sfendeno per mezo e liga l'una in chavo del'altra, e fa-le longe ben trexento a passa. E sono più forte che non serave sartie de chanevo per tirar.

TB 7218 Le navi non ànno sartie di canape se no a la vela e a l'albero, ma ànno pilotte di canne, colle quali si tirano le navi su per questo fiume.

Canapinis funibus non utuntur nisi pro malo navis et velo eius, sed pilorcas faciunt de arundinibus magnis, de quibus supra facta est mentio,

PII 594 que habent .XV. passus longitudinis; cum his pilorcis quandoque naves per flumina trahuntur, scinduntur enim arundines et scissuras ligant in simul et huiusmodi funes longos faciunt valde; nam alique pilorce habent longitudinem passum trecentorum et fortiores sunt funibus canapinis.

Non àno sartie de chanevo ma le sartie soe sono fate delle chane dele qual per avanti ò parlado; e de quello ne fano sartie fendendolle e lligalle insieme;

VB 1097 e fano de quelle tortice longe .II'c. passa, le qual sono più forte che non sono le tortiçe de canevo et sono ben fate e per simelle tute alltre sartie besogni per le nave. 
Item naves non habent omnia paramenta de sartiis canapi, nisi solum pro arboribus et velis. Restam quidem habent de canis cum qua trahuntur per flumen. Et iste cane sunt arundines longitudinis decem pasuum, quas homines scindunt per medium et, ligantes capud unum cum alio, faciunt eas longas bene de .CCC. pasibus; et multo fortiores sunt quam canapum. Et quelibet istarum navium habet.VIII. vel .X. vel .XII. equos qui trahunt ipsas per flumen.

\section{Tra gli elementi notevoli del passo, segnalo i seguenti:}

1. il termine pelorce, che designa una «fune lunga e sottile adoperata per tirare a terra una rete da pesca» (Battaglia 1961-2002, s.v. «spilorcia»), ${ }^{18}$ ha messo alla prova i copisti, che hanno reagito di fronte alla difficoltà lessicale in modi diversi: (a) riproducendo la parola che compariva nel modello (talora con qualche incertezza di trascrizione), e corredandola all'evenienza di una glossa esplicativa, come avviene in VA ( «i àno pilore 0 , per dir chiaro, alzane de chane»); la glossa non compare né in P II 59 né in TB 72, che derivano da VA e leggono rispettivamente pilorcas e, con banalizzazione, pilotte; ${ }^{19}(\mathrm{~b})$ riconducendo, su base analogica, il lemma pelorce a uno equivalente, ma più consueto, come avviene in $\mathrm{Z}$ e in $\mathrm{V}$, che leggono restam/resti («cavo di giunco o di canapa usato in marina», cf. Battaglia 1961-2002, s.v. «resto $\left.{ }^{3} »\right) ;{ }^{20}$ (c) aggirando l'ostacolo, e facendo sparire il 'corpo

18 L'etimologia del lemma, di diffusione prevalentemente toscana, e il suo legame con l'aggettivo spilorcio sono indagati da Nocentini (1993), secondo il quale il ritardo con cui i termini dialettali e marinareschi vengono registrati nei dizionari spiega perché il significato traslato ('avaro, tirchio') preceda nella documentazione quello concreto. Il pelorce poliano (la forma in s- «è derivata con l'aggiunta di una s- intensiva» secondo Nocentini 1993, 2) ha buone possibilità di rappresentare la prima occorrenza nota di questi «cordami più economici, realizzati con fibre vegetali povere, come la paglia e lo sparto, e con le striscioline pelose di pelli di animali» (2-3). Cortelazzo e Zolli (1999) registrano la forma «spilorcio» come problematica: «va poi detto che nemmeno di pilorcio (attest. dal sec. XV: TB) è conosciuta l'etimologia (non essendo accettabile la proposta del DEI, che rinvia a pelo e palorcio, fune lunga e sottile per tirare a terra una rete)».

19 Non è chiaro cosa designi il termine nel contesto: se non è una trascrizione inerziale, si tratta forse di una forma da ricondurre a «pilota» (per indicare un'imbarcazione usata per le operazioni di traino?). Il lemma «pilòtta ${ }^{1}$ » (Battaglia 1961-2002, s.v.) è attestato in area senese per indicare un «manicotto di velluto usato per spianare il pelo dei cappelli di feltro durante la stiratura», ma tale accezione non è, evidentemente, pertinente nel caso in esame.

20 Battaglia (1961-2002), s.v. «rèsto ${ }^{3} »$ : «lungo cavo di canapa per trascinare le reti nella pesca d'altura (sec. XIV, Esposizioni dei Salmi, il m. resto in un doc. ven. del 1311: Stussi). Lat. rĕste $(m)$, rĕsta $(m)[. .$.$] . Il senso di corda, cavo, pare il più ant. ed è testimoniato in tutti i secoli$ della nostra storia linguistica, dal sec. XIV (ma anche prima, se nel Conto navale Pisano, scritto tra l'XI e il XII sec., appare già il der. restaiolo) al XX». Più articolata la voce nel Dizionario di marina (Falqui, Prati 1937): «Grosso cavo di canapa lungo 800-1000 metri, legato per un'estremità ad una parete di una rete a strascico e per l'altra al palo che è a prua della barca e che 
del delitto', soluzione adottata da L (e maggioritaria nella tradizione extra-ramusiana: cf. Fr 146, Kc 63, TA 143); (d) eliminando in un primo tempo il dettaglio, ma recuperando poi un termine più o meno equivalente: così avviene in Ramusio, debitore a VB sia per la struttura del periodo che per il materiale informativo, da cui è tratta l'innovazione «tortizze» (una grossa fune ricavata dall'intreccio di più corde sottili); ${ }^{21}$

2. un ulteriore elemento differenziale è costituito da un'informazione relativa all'impiego di cavalli per trainare le navi dalla terraferma, attestata solamente in R II 63 8; Z 80 23; e, in forma abbreviata, in V 728 (la pericope è indicata con il sottolineato). Al di là dell'aspetto testuale, l'aggiunta, che isola $\mathrm{R}, \mathrm{Z}$ e V, presenta qualche problema di pertinenza storica, se è vero, come sostenuto da Yule e Cordier (1903, 2: 174), che questa è l'unica attestazione dell'uso di cavalli da traino lungo il Fiume Azzurro. ${ }^{22}$ L'esempio consente di mettere in rilievo un'altra difficoltà, che il Milione condivide con il genere odeporico: alla necessità ovvia di combinare informazioni di tipo linguistico, storico e critico-testuale si somma l'importanza di connettere documentazione orientale e occidentale, il che non è sempre facile; molti studi cinesi recenti non sono tradotti in inglese, mentre i lavori di ambito linguistico-filologico, soprattutto in italiano, rimangono sconosciuti al di fuori dell'Europa. ${ }^{23}$

serve per manovrare la rete quando viene messa in bando o salpata, oppure per assicurarla alla barca durante il traino. [...] - Resto (venez.): cavo fatto di sparto o di giunco. Resti zoe (cioè) sartia de herba si legge pure nel Capitolar della Tana. [...] Restus in tal senso anche nel basso latino (1246), e Rest. nel catalano»; infine cf. Boerio 1867, s.v. «resta»: «detto in T. de' Pesc. Sferzina. Quella corda che, attaccata alla rete delle bilancelle, serve come per allungarla e tirarla in terra, e viene a far lo stesso servizio che la spilorcia per la rezzola».

21 Come documentato nella voce curata per il glossario in corso di caricamento nel database (Rinaldin, in corso di stampa), quella di VB è «una delle prime occorrenze attestate del significato di uso marinaresco (Sella 1944 [...], 587, riporta un contesto latino di produzione veneziana del 1364: «<marinarii> sorserunt duo ferra, unum de tortiza, alterum de resta»), seguito da Pietro Querini, a. 1430 (GDLI)».

22 «The tow-line in river-boats is usually made (as here described) of strips of bamboo twisted. Hawsers are also made of bamboo. Ramusio, in this passage, says the boats are tracked by horses, ten or twelve to each vessel. I do not find this mentioned anywhere else, nor has any traveller in China that I have consulted heard of such a thing». Più conciliante Marsden (1818, 497-8 nota 981): «At the present day it would seem that vessels of every description are tracked by men only, and not by horses, which, as well as other cattles, are to a certain degree, but there is reason to believe that under the Mungal princes, great numbers were brought from Tartary, and much encouragement given to breeding them». Ė invece ben documentata l'usanza di trainare le barche a braccia; cf. Ménard (2001-09, 5: 146 note 40-50) con relativa bibliografia.

23 È questo uno dei problemi discussi nel recente convegno su Marco Polo organizzato da Ulrich Vogel e dai suoi collaboratori a Tubinga (10-11 ottobre 2017), che ha cercato di far dialogare filologi e orientalisti occidentali con specialisti orientali, con l'intenzione di avviare in futuro progetti condivisi, che mettano a profitto le diverse competenze. 
Tornando al nostro passo, riassumo come possiamo accedere ai dati fin qui presentati attraverso il Ramusio digitale.

Cliccando su 'Libro II' si apre un menu, contenente l'elenco dei capitoli. Il capitolo su Singui è R II 63: abbiamo la possibilità di muoverci nei capitoli precedenti e in quelli successivi. Il testo si presenta su due colonne: sulla sinistra abbiamo il testo ramusiano, sulla destra la fonte o le fonti che il responsabile del capitolo (in questo caso Alvise Andreose) ha giudicato preponderante; quando, come nell'esempio che ci interessa, la colonna di destra è vuota, significa che il lavoro di intarsio svolto da Ramusio è stato talmente fitto da rendere difficile il riconoscimento univoco di una fonte.

Dalla nota titolo, R II 63, si accede a una scheda informativa articolata su due livelli: (a) le concordanze con le altre redazioni; da qui è possibile spostarsi sul passo corrispondente in $\mathrm{F}, \mathrm{V}, \mathrm{Z}, \mathrm{VA}, \mathrm{P}, \mathrm{VB}, \mathrm{L}$; in questo modo il lettore può stabilire velocemente una sinossi tra i testimoni, oppure leggere il capitolo nella redazione che preferisce, o ancora, cliccare sul titolo del capitolo scelto e verificare come si è svolta la costruzione del capitolo da parte di Ramusio.

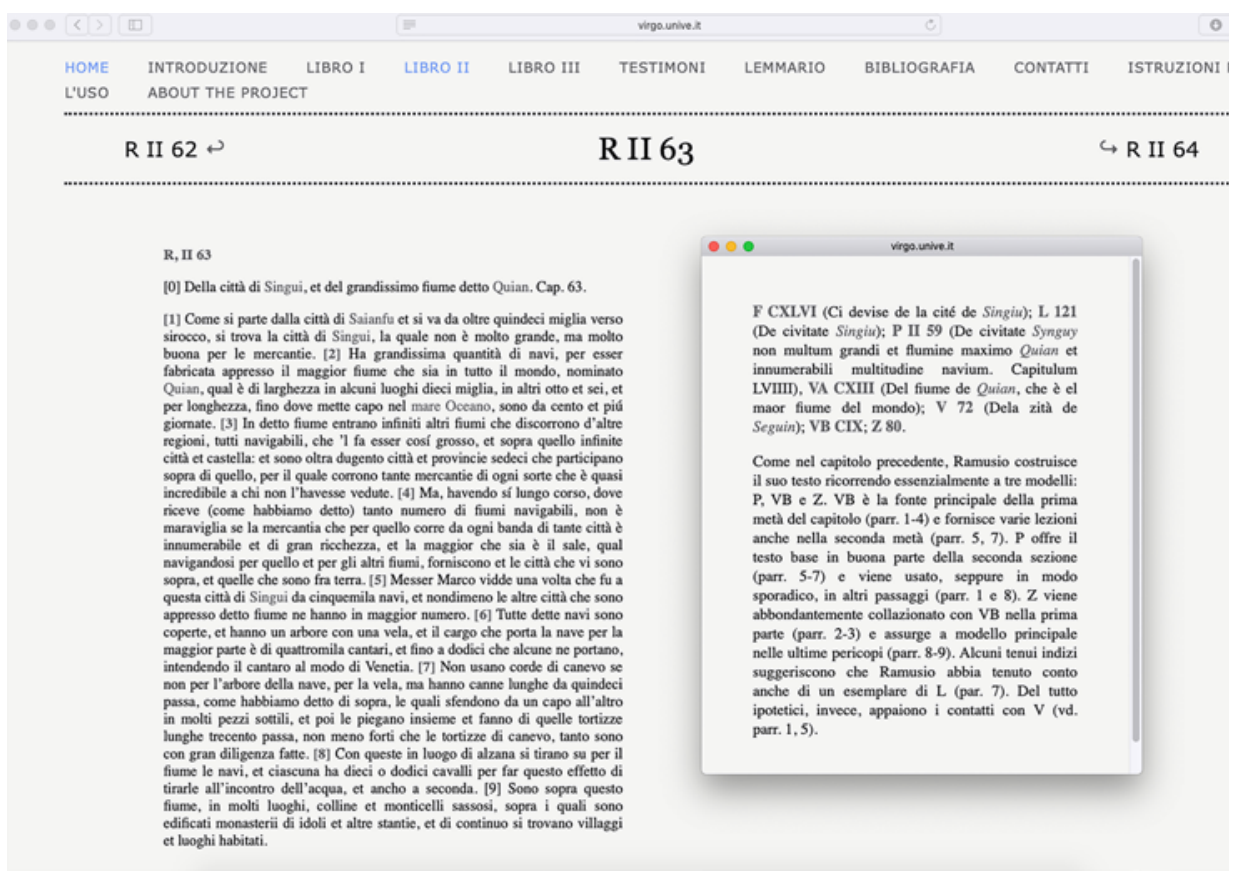

Figura 3. Il capitolo R II 63 nel Ramusio digitale 
La rubrica liminare presenta due lemmi marcati (il toponimo Singui e l'idronimo Quian): cliccando sul primo, si accede alla scheda dedicata alla città, con una spiegazione della sua funzione nell'itinerario poliano e la localizzazione in Google Maps. La scheda-toponimo è strutturata su quattro fasce: una prima fascia contiene l'elenco delle occorrenze in R; una seconda fascia funziona da tavola delle corrispondenze con le altre redazioni; una terza fascia offre la bibliografia di riferimento; per finire con la voce enciclopedica vera e propria, mirata ed essenziale.

All'interno del capitolo ogni paragrafo è commentato. Le schede sono 'flottanti' e si possono affiancare, ridimensionare, stampare. Nel caso in esame, il curatore del capitolo segnala in particolare alcuni passaggi: nella parte esordiale Ramusio segue il suo modello P; per alcuni apporti si sospetta la provenienza da $\mathrm{L}$, mentre è sicuro, alla fine della pericope, il ricorso a VB, da cui è tratto, come abbiamo visto, il termine tortizza, e forse «anche l'omissione del riferimento all'uso delle alzaie per trainare le navi, che P F V e Z collocano all'interno di questo brano». Nel paragrafo successivo leggiamo che Ramusio combina la lezione di $\mathrm{P}$ con quella di Z: «Il riferimento all'uso dei cavalli figura in forma abbreviata anche in V 72 7-8 [...]. Nelle altre redazioni poliane non se ne fa menzione».

Il database prevede inoltre altre sezioni, accessibili dalla homepage: (a) l'«Introduzione», che contiene la storia del progetto, le sue finalità, l'elenco dei collaboratori, ed è suddivisa in cinque paragrafi dedicati tanto all'inquadramento complessivo (Andreose, Barbieri, Burgio, Mascherpa e Simion) quanto ad aspetti specifici, come la lingua e lo stile ramusiani (Romanini) o il rapporto del testo ramusiano con la cartografia (Cattaneo); (b) la lista dei «Testimoni», che permette al lettore di leggere una sola delle fonti; ${ }^{24}$ (c) il «Lemmario», pensato come un aggiornamento bibliografico delle ricerche sull'Asia poliana, con riferimento in particolare ai due repertori tradizionali: Pelliot (1959-73) e Cardona (in Bertolucci Pizzorusso 1975, 488-761); (d) è inoltre pronto, come accennavo, un glossario (Rinaldin, in corso di stampa) per i realia notevoli del testo di R. Tutte le sezioni del Ramusio digitale sono implementabili e aggiornabili: è possibile inviare segnalazioni, testi, correzioni, materiali aggiuntivi, caricabili nel database previa valutazione; (e) chiude l'opera una «Bibliografia», limitata alle opere citate nell'ipertesto.

24 Nel Ramusio digitale abbiamo reso disponibili per la prima volta i testi dell'epitome latina $\mathrm{L}$ e delle redazioni veneziane V e VB; la trascrizione di R è stata condotta sulla editio princeps del 1559 (l'edizione Milanesi 1978-88 si fonda sulla ristampa del 1583); la trascrizione interpretativa di P si basa sulla lezione del manoscritto Ricc. 983 della Biblioteca Riccardiana di Firenze. Malgrado la sua importanza nella storia della ricezione dell'opera, ricordo che manca ancora un'edizione di P basata sull'insieme dei suoi relatori; prima della messa online della trascrizione del codice Ricc. 983, si poteva ricorrere alle edizioni di Prášek (1902) e Iwamura (1949). Come ho già segnalato, abbiamo incluso nel database solo le redazioni sicuramente utilizzate da Ramusio (Z, VB, P, e VA in quanto modello di P) con l'aggiunta di V, L e F (quest'ultimo utilizzato come testo di riferimento). 


\section{Limiti della mise en page tradizionale}

Questi, in sintesi, i dati cui può accedere il lettore. Credo appaia evidente che un'edizione tradizionale avrebbe reso impossibili la molteplicità e la simultaneità delle operazioni qui realizzabili in tempi rapidissimi. Allo stesso tempo un'edizione di questo tipo rappresenta un buon compromesso tra 'scientificità' e divulgazione, nel senso che i complementi esegetici (commento, lemmario, glossario) guidano il lettore comune in un testo complesso senza imporgli un corpo a corpo con note e apparati. Il supporto digitale presenta certamente anche alcuni svantaggi, in particolare la necessità di manutenzione costante: il server può diventare obsoleto, e richiedere la migrazione dell'ipertesto; i collegamenti possono essere minati da malfunzionamenti, ed è necessario intervenire periodicamente (cf. Italia 2016b, 252). D'altra parte i vantaggi del vettore si misurano bene verificando la struttura delle due edizioni integrali già citate, Benedetto (1928) e Moule, Pelliot (1938): ${ }^{25}$

1. l'edizione di Benedetto (1928) assume come testo-base F, normalizzandone la grafia; la seconda fascia d'apparato raccoglie le lezioni ritenute autentiche, desunte specialmente dalla «famiglia B»: nel caso in esame (140), la pericope sul traino delle imbarcazioni riportata in $\mathrm{R}$ e in $\mathrm{Z}$ si legge nella nota (d). Come si può osservare, questa forma editoriale tende a sacrificare l'individualità dei singoli relatori: così, ad esempio, la lezione di $\mathrm{V}$, che reca, seppure in forma brevior, la medesima notizia, viene espulsa dall'apparato. La fissità della carta obbliga Benedetto a soluzioni 'miste', nella necessità di tenere insieme quante più informazioni possibili; la prima fascia d'apparato diventa così il bacino di raccolta di materiali variegati: dalle letture scartate («10. opp. longe»), alla discussione di problemi testuali ( «13 Forse $\mathrm{XV}^{\mathrm{m}}$, come LA e TA»); fino a proposte lessicali, come l'etimologia di pelorce: «23 VA interpreta pelorce come sinonimo di alzane [...]; e similmente P parla delle pilorchae; la parola è probabilmente da considerarsi come un composto di orce (= ital. orza nel senso originario di fune); TA fa pensare a un 'ele ont [le petite] de pelorce de canne' (da connettere con pilorcio attestato col senso di ritaglio di pelle, senso conciliabile con quello di strisce di scorza?)». ${ }^{26}$

25 Benché Pelliot figuri come co-editore, la responsabilità del testo critico va ascritta al solo Moule; i due volumi con il commento e gli indici, affidati a Pelliot, non furono infatti mai pubblicati (i materiali preparatori uscirono postumi tra il 1959 e il 1973 con il titolo di Notes on Marco Polo, per le cure di Louis Hambis).

26 Benedetto basa verosimilmente la sua proposta etimologica sul Tommaseo, Bellini 186179, s.v. «pilorcio» (cf. nota 19): «S. m. Si chiamavano Pilorci dai pellicciai Quei ritagli di pelle, che, non essendo buoni a mettere in opra, gli riducono in spazzatura, la quale poi 


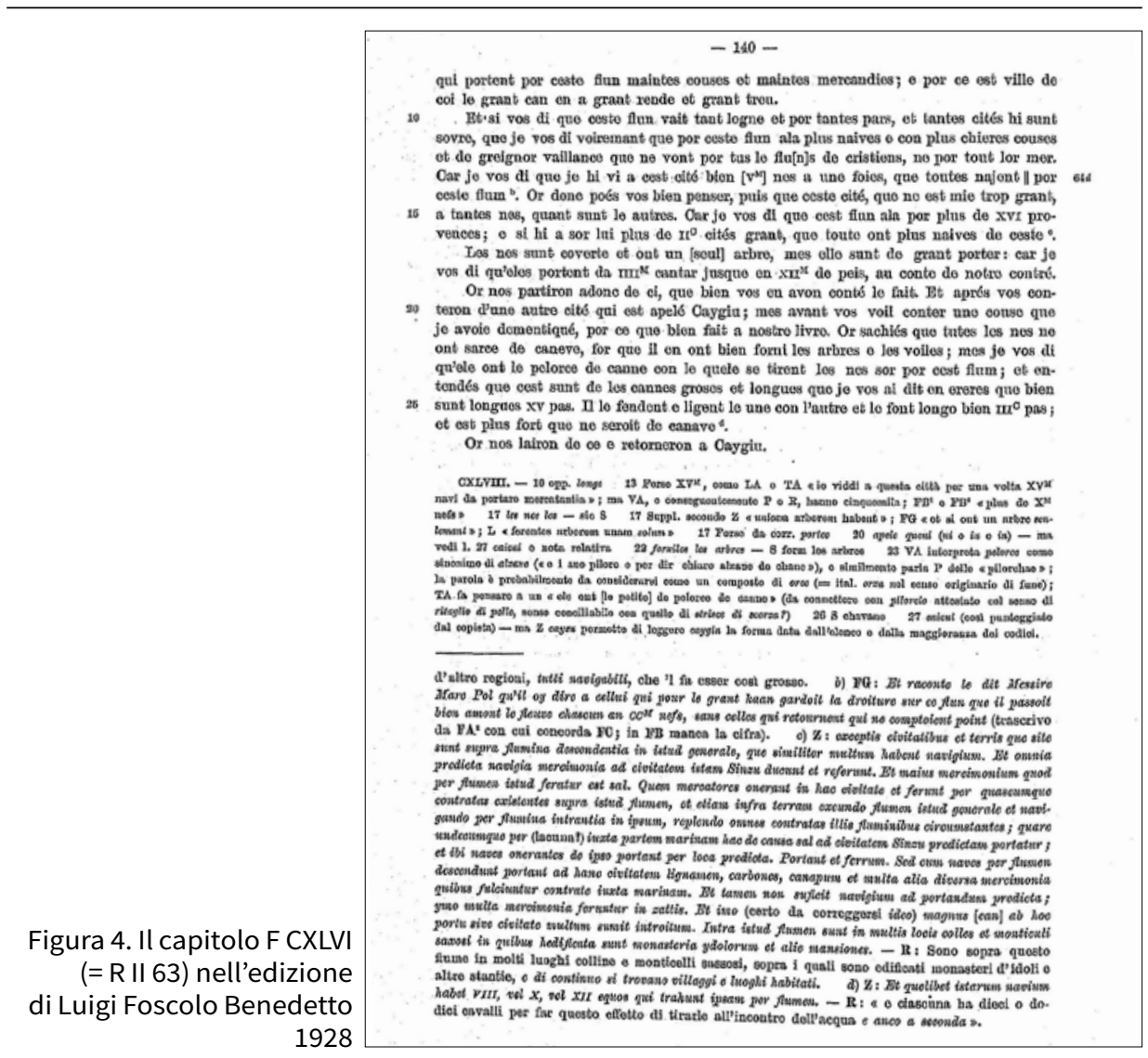

2. L'edizione allestita da Moule e Pelliot (1938) innesta la varia lectio, in corsivo, sul dettato di $\mathrm{F}$, tradotto in inglese; la provenienza dei singoli apporti è indicata con sigle a margine. Il limite maggiore dell'operazione risiede nella mancanza di commento: non viene mai

vendono per governar i terreni». Il Tommaseo, Bellini attesta però anche l'uso marinaresco del lemma, s.v. «spilorcio»: «S. f. e m. Term. de' Pescatori. Sottile e lunga fune, corredata di distanza in distanza di sugheri, con cui si tira a terra la sciabica e la rezzuola». Anticipo che nel commento inedito all'edizione postuma (Simion 2016), in preparazione con Irene Reginato, Benedetto esprime una posizione diversa: «Pelorce est enregistré par Godefroy comme un substatif masculin et défini par lui 'corde pour le halage des vaisseaux', mais sa seule citation est ce passage [...]. Il s'agit presque sûrement d'un mot d'origine grecque dont on trouve des parents, à des époques diverses, dans plusieurs parlers italiens. Parmi les mots vivants de la même famille on peut citer le calabrais palorciu, qui désigne la corde avec laquelle on tire une embarcation vers la rive». 
THE DESCRIPTION OF THE WORLD $\$$ THEIR SIZE AND RIGGING at once which all sail $[64 d]$ by this river, wbich is so broad that it dos not seem to be a FB river but sea, it is so broad. Now then you can well think, since this city which is not at all too large has so many ships, how many are the others. For I tell you that by this river one goes through more than sixteen provinces, and also there are $\mathrm{v} v$ more than two hundred wery large cities upon it, witbout the towns and the villages, L. FB which are all larger than this of Singiu and all have more boars than this, not counting $v_{A} \mathrm{Z}$ the cities and lands which are situated on the riwers whicb flow down into this main river, wbicb likewise bave mucb sbipping; and all the aforesaid sbips bring merchandist to this city of Singiu and carry it back; and the chicf nerchandise wbich is carried on this river is salt, whicb the mersbants load in this city and carry tbrougb wbatever regions are upon tbis river, $E$ also inland, leaving this main river and sailing by the rivers wbich enter it, filling all the regions around those rivers. For for this reason salt is carried to this aforesaid city of Singin from everywbere about the sea shore, and there the sbips loading earry of it througb the foresaid places. They also carry iron. But wben the ships come down by tbe river they bring to tbis city wood, chartoal, bemp, and many otber different wares with which the regions near the sea sbore are supplied. And yet the sbipping is not enough to carry the aforesaid tbings; indeed many goods are carried on rafts. - And all the large ships of tbis river -are made as I sball tell L VA Z you. Thry are covered with only one deck and have only one mast witb one sail, but they V L R are of great tonnage, for I tell you that they carry cargo for the most part from 4000 a cantar up to 12000 (wbicb some of them carry) in weight by the count of our country $R$ of Venest, -varying between the said numbers acrording to tbe size of the sbip. Now there is R P FB notbing else wbich does to tall, and so we will depart then from here, of which we have well told you the facts, and afterwards we will tell you of another city which is called Caigiu. But first I wish to tell you a thing which I had forgotten to write FB because it does well for our book to tell. Now you may know that all the ships FB have not all tbe tackle of ropes of hemp, except indeed that they have the masts and $z$ the sails rigged with them. But I tell you that they have the hawsers or, to spack vi plainly, tow-lines of notbing clse but of canes, with which the ships are towed upstream FB by this river. You may know that these sbips whicb go on tbis river, those wbich go against FB the current of the water are towed because the current of the water is strong, otherwisc tbey could not go. And you understand that these canes are the thick and long canes of $\mathrm{z}$ which $I$ have told you in this book above, which are quite fifteen paces long. They FB take tibse canes and split them from one end to tbe otber into many tbin strips and bind them FB $R$ the one end with the other and make them ropes as long as they wisb, twists quite $\mathrm{Z}$ FB $\mathrm{R}$ three hundred tlls, tbat is paces, long; and it is macb stronger than hempen ropes LT $z$ VA would be, witb so great care are they made. And cach of these sbips bas eigbt or ten or twelve $\mathrm{R} \mathrm{Z}$ borses whicb tow it through the river-against the stream, and also witb it. At many places $\mathrm{R} Z \mathrm{Z}$

Figura 5. Il capitolo 147 (= R II 63) nell'edizione di Moule, Pelliot 1938

chiarito da Moule quali e quanti siano i testimoni utilizzati, e perché. Così, troviamo inglobate nel testo critico, senza alcuna indicazione, un'amplificazione di LT (redazione contaminata tràdita da un solo testimone, il manoscritto lat. 3195 della Bibliothèque nationale de France): ${ }^{27}$ «ells, that is paces» (traduzione di «bene trecenta brachia, imma passus»); e viene trattata come originale la glossa di VA: «hawsers or, to speak plainly, tow-lines».

27 Si tratta di un testo che contamina TA e P, pubblicato in appendice all'edizione di $\mathrm{F}$ da Roux de Rochelle (1824), e ora oggetto della tesi di dottorato di Vito Santoliquido (che ringrazio per avermi fornito la pericope citata, che si legge a c. 50c). Su di esso si vedano Bertolucci Pizzorusso (1975, 335-7) e Gadrat-Ouerfelli (2015, 31-5). 
In entrambi i casi insomma, pur se con modalità diverse, la forma editoriale apparentemente nitida e di immediata decifrazione nasconde delle insidie: (a) il lettore non può falsificare le ipotesi dei due editori, a meno di recuperare da sé tutti i codici e le edizioni citate in forma frammentaria nei due apparati; (b) l'esito è un testo virtuale, in cui F è costretto a incorporare una miriade di frammenti in lingue diverse dall'originale, senza che sia possibile valutare pienamente l'apporto di ciascuna attestazione, e quindi il suo specifico significato.

\section{Una nuova ipotesi stemmatica per il Devisement dou monde/ Milione}

Mi riferisco alla categoria di falsificabilità, da intendersi non tanto come vessillo astratto di scientificità, quanto come esigenza di presentare con chiarezza le ipotesi di interpretazione del testo e della tradizione, in modo da permettere al lettore di valutarne l'attendibilità e di proporre eventualmente soluzioni alternative. ${ }^{28}$ Non ci aspettavamo però di essere noi i primi a elaborare una classificazione stemmatica differente da quella di partenza. Presentiamo qui per la prima volta la nuova ipotesi di lavoro, grazie anche all'occasione di discussione offerta da questo convegno, avvertendo che il riassestamento riguarda principalmente il ramo $\beta{ }^{29} \mathrm{Il}$ processo di progressivo impoverimento del contenuto individuato da Benedetto riflette le dinamiche interne a $\delta$, ma non risulta altrettanto efficace per $\beta$, in cui è attivo un dinamismo di segno opposto, per innesto di incrementi successivi. ${ }^{30}$

Ho segnalato alcune delle insidie a cui espone l'individualità delle testimonianze di F, Z e V; in effetti è proprio lo studio della tradizione indiretta di Z (in particolare dei passi poliani contenuti nelle due compilazioni domenicane di Pietro Calò da Chioggia e di Filippo da Ferrara studiati da Mascherpa 2007-08 e 2008, e da Gobbato 2015, cui farò riferimento con le sigle $\mathrm{Z}^{\mathrm{f}}$ e

28 Cf. Stussi (1994, 20-1): «il lettore dev'essere messo in grado di verificarla [l'edizione critica] punto per punto ed eventualmente di dissentire»; Leonardi $(2017,48-9)$ : «il principio [...] potrebbe tradursi in filologia nella necessità che le ipotesi presentino, e via via discutano, anche gli elementi atti a falsificarle. Una concreta applicazione in ambito stemmatico potrebbe essere l'invito a esporre i dati che entrano in gioco nella classificazione dei manoscritti non solo secondo l'ipotesi stemmatica individuata, ma anche secondo le altre plausibili».

29 La nuova proposta è il frutto di una riflessione collettiva e della condivisione di dati e ipotesi, in particolare con Alvise Andreose, Eugenio Burgio, Mario Eusebi e Giuseppe Mascherpa.

30 L'ipotesi del progressivo accrescimento del testo per innesto di varianti d'autore è stata affacciata in passato, tra gli altri da Bertoni 1928, Battaglia Ricci (2001, VIII-IX, XI-XII); Ménard (2001-9, 1: 17-9), più sulla base di prove indiziarie che di una vera ricognizione testuale. Si veda ora l'intervento di Giuseppe Mascherpa in questo volume. 


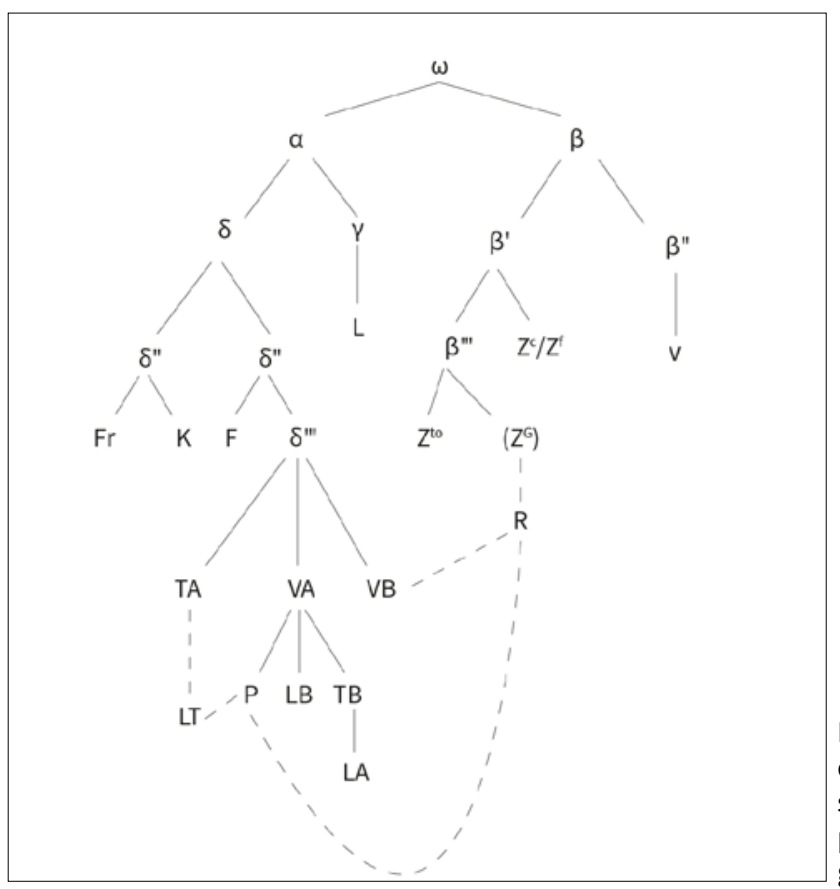

Figura 6. Stemma codicum del Devisement dou monde secondo la proposta presentata a Saarbrücken, 23 giugno 2017

$Z^{c}$ ) che ha incrinato l'idea che lo $\mathrm{Z}$ toledano testimoni la fase più antica e conservativa dell'opera poliana. ${ }^{31}$ Lo $\mathrm{Z}$ usato dai due domenicani si dimostra molto più fedele a $\mathrm{F}$, a livello di lessico, sintassi, manipolazione dei contenuti, di quanto non faccia il gruppo toledano-Ghisi; si profila così l'esistenza di una divaricazione interna a $\beta$ : da una parte uno $\mathrm{Z}$ brevior complessivamente fedele a $\mathrm{F}$, ma con in più alcuni incrementi; dall'altra uno $\mathrm{Z}$ caratterizzato da aggiunte più corpose e da un certo grado di rielaborazione formale.

Più in particolare, Calò deriva da Marco Polo il racconto della morte e dei miracoli post mortem dell'apostolo Tommaso in India. ${ }^{32}$ Nella tradizione

31 La redazione $\mathrm{Z}$ non coincide infatti con la testimonianza del solo toledano, ma è rappresentata da una tradizione indiretta che comprende (oltre al codice Ghisi utilizzato da Ramusio), (a) il codice a disposizione di Fra Mauro per l'allestimento della sua mappa mundi (ante 1453, conservata presso la Biblioteca nazionale Marciana; cf. Burgio 2009, 92-4; Burgio 2014); (b) il codice utilizzato dal domenicano Pietro Calò da Chioggia, autore di una compilazione agiografica nota come Legendarium o Legendae sanctorum (redatta verosimilmente tra 1332 e 1340; cf. Gadrat-Ouerfelli 2015, 173-4; Mascherpa 2007-8 e 2008); (d) gli estratti poliani inseriti da Filippo da Ferrara O.P. nel suo Liber de introductione loquendi (redatto tra 1325 e il 1347), forse a partire dallo stesso antigrafo a disposizione del confratello Calò (cf. Gobbato 2015).

32 Il testo di Calò in Devos (1948, 270-2) e Gadrat-Ouerfelli (2015, 414-16); per Filippino cf. Dutschke (1993, 1227-59) e Gobbato (2015, 319-68). 
la leggenda si compone di due passaggi: (a) la descrizione della sepoltura del santo in una città del Maabar, seguita dal racconto della sua epifania contro un barone locale, che aveva empiamente adibito a granaio gli alloggi tradizionalmente usati dai pellegrini (F CLXXV 2-11); (b) la narrazione della morte di Tommaso, colpito dalla freccia scoccata per errore da un cacciatore di pavoni (F CLXXV 12-16). La versione di Z toledano (109 1-36) aggiunge una digressione sulle piantagioni di noci di cocco, per le quali i cristiani della regione pagano un tributo al rex locale (§§ 5-12), ed elimina il personaggio del 'barone', indicando nel re la minaccia per i pellegrini. Già Benedetto (1959-60, 55-7) aveva osservato che il Legendarium contiene un rinvio alla partizione del suo antigrafo ( Dominus Marcus Paulus Milionus de Venetiis in libro suo, capitulo CLXXV»), che non coincide con quella attestata nel toledano (in cui la morte del santo occupa il cap. 109). Mascherpa (2007-08, 85-8) ha interpretato la discrepanza di numerazione (in questa e in altre citazioni poliane da parte di Calò) come la conferma dell'esistenza di copie di $\mathrm{Z}$ più complete della toledana e dotate di un'articolazione interna più affine a $\mathrm{F}$ (dove il capitolo in esame occupa appunto la posizione CLXXV). Lo Z usato da Calò parla inoltre di un «Baro illius contrate», come F CLXXV 5 «baron de celle contree», contro il «rex» del toledano. Di conseguenza pare molto probabile che la pericope sulle noci di cocco, i riferimenti al tributo e l'ingiunzione del santo siano stati introdotti nel toledano (o nel suo modello) ${ }^{33} \mathrm{~A}$ fronte della fedeltà complessiva alla versione di $\mathrm{F}$, l'accordo in una variante significativa permette però di ricondurre con sicurezza il modello di Calò al ramo Z: l'indicazione che l'apostolo viene colpito mortalmente in tibiam dexteram (Z 109 33), e non emi le destre costee (F CLXXV14). ${ }^{34}$

Più numerose sono le occorrenze poliane nel Liber de introductione loquendi di Filippo da Ferrara, sempre riconducibili a un esemplare Z;

33 Z 109 12, 22, 26. Mascherpa (2008, 179-80) individua anche il possibile punto di partenza dell'interpolazione: «A ben guardare, l'intervento di Tommaso contro la tassa e la sua successiva abolizione - ovvero, i due ampliamenti peculiari di $\mathrm{Z}^{\text {to }}$ [lo $Z$ toledano] conseguenti alla sostituzione del baro con il rex - sono introdotti nel capitolo dalla medesima locuzione avverbiale, vale a dire de cetero 'd'ora in avanti': sotto il profilo della struttura testuale, la locuzione parrebbe proprio fungere da giuntura - quasi da introduttore di una glossa - tra lo scheletro del testo dello $\mathrm{Z}$ originario e l'innovazione abilmente incastonata, opera di un non inesperto interpolatore, che, istituendo una liaison tematica tra la sequenza dell'aneddoto miracoloso e la digressione erudita, ha scardinato il caratteristico procedere 'per blocchi' della pagina poliana, e ha conferito al passo l'aspetto di un compatto exemplum moraleggiante».

34 Benché la variante non sia patentemente erronea, mi pare che alcune considerazioni esterne inducano a giudicarla come un errore: il costato è un punto chiave nella vicenda dell'apostolo (la sua mano, elemento chiave di tutte le tradizioni agiografiche, orientali e occidentali, tocca il costato di Cristo risorto), e quindi la ferita nel destre costee del santo acquista un valore di contrappasso piuttosto trasparente, a differenza della variante con la tibia. Non è ancora chiaro come si sia prodotta quest'innovazione, probabilmente ascrivibile all'ambiente domenicano. Cf. Mascherpa in questo volume. 
l'analisi degli excerpta poliani condotta da Gobbato (2015, 341-56), offre ulteriori conferme dell'attivismo interno del codice toledano, e, per contro, della fedeltà a $\mathrm{F}$ a livello di forma e sostanza dello $\mathrm{Z}$ brevior. ${ }^{35}$

La soluzione più economica, faute de mieux, è quella rappresentata nel nuovo stemma che stiamo testando, e che spiega tanto le differenze tra $\alpha$ e $\beta$ quanto la divaricazione interna a $\beta$ con la possibilità di varianti redazionali: gli incrementi sarebbero stati inseriti, per stadi successivi, su un testimone franco-italiano simile a F, probabilmente a Venezia, dopo il ritorno di Marco Polo dalla prigionia genovese. La nostra capacità di analisi è tuttavia limitata (almeno allo stato attuale della documentazione) dallo stato frammentario della tradizione indiretta, mentre l'apporto che potrebbe venire da $\mathrm{V}$, la redazione che presenta più affinità con $\mathrm{Z}$, ma che condivide anche molte caratteristiche con $\delta$, è ridotto nella sua portata dalle cattive condizioni del testo e dalla mancanza di errori congiuntivi davvero sicuri: per lo più $\mathrm{V}$ condivide con $\mathrm{Z}$ lezioni caratteristiche; e poiché $\mathrm{V}$ ha alle spalle un ipotesto latino, gli accordi con il toledano si possono spesso giustificare (almeno in linea astratta) anche pensando a convergenze poligenetiche di traduzione. Nell'esempio di R II 63, il fatto che Z e V rendano entrambi il lemma pelorce con «resta» (restam/resti) non permette di trarre conclusioni genealogiche definitive: il sintagma "pilorciariam sive restam» si trova attestato (Roma, 1363), in un contesto analogo, anche in Sella (1944): «pilorciaria, palafitta: ad trahendum pilorciariam sive restam in flumine». È quindi lecito chiedersi quante possibilità di resa avesse un traduttore mediolatino di fronte a un lemma come pelorce. D'altra parte la quantità e la continuità degli accordi di $\mathrm{V}$ con $\mathrm{Z}$ è tale da costituire una serie, e da lasciare così aperte solo due vie: la dipendenza di $\mathrm{V}$ da $\beta$ (dal $\beta$ brevior identificabile grazie alla tradizione indiretta di matrice domenicana) o la contaminazione. ${ }^{36}$

35 Una variante significativa marca una divaricazione interna al ramo $\beta$ : in F CXVIII 12 i coccodrilli della regione di Carajan imprimono sulla sabbia delle impronte, paragonabili a quelle prodotte dal rotolamento di una botte di vino («ele fait si grant fousee en sablon qu'il senble qe soit voutee une bote de vin plene»); a bote (attestato in Filippino, I 107 6: «faciunt foveam in arena per quam vadunt, ac si ibi fuisset revoluta una magna veges plena vino»), $\mathrm{Z}$ toledano 5720 oppone trabs 'trave', che ha tutta l'aria di un'innovazione secondaria («tam magna patent vestigia quasi una magna trabs illac foret deducta»); trabs doveva essere la lezione attestata anche nel codice Ghisi, dato che R II 408 legge «per la troppa gravezza del peso loro appaiono i vestigii così grandi come se una gran trave fosse stà tirata per quell'arena».

36 Infatti, «a parità di debole forza congiuntiva, non sarà lecito ritenere più valida una lunga serie di errori piuttosto che una esigua? Infatti l'errore debolmente congiuntivo è intrinsecamente poligenetico, sicché in teoria sia la serie breve che quella ampia potrebbero essere casuali, ma è evidente che ciò è tanto meno probabile quanto più la serie è lunga. È vero che in critica testuale non si dovrebbe far conto di elementi che possano essere fortuiti, ma se non ce ne sono altri dobbiamo pur mettere a frutto quelli che abbiamo» (Varvaro [1970] 2004, 589-90). 


\section{Verso un «Marco Polo integrale»}

Accanto a questo risultato 'colposo', il Ramusio digitale ci ha permesso di mettere alla prova la forma ipertestuale e di definire una serie di correttivi tecnici necessari all'allestimento di un nuovo progetto di edizione integrale del Milione $:^{37}$ il nuovo testo critico sarà «integrale» relativamente alla fisionomia e al contenuto del testo, e sarà in inglese, come pure l'introduzione, il commento, il lemmario e il glossario, mentre le singole redazioni rimarranno nella lingua che le ha tramandate. Ancora una volta, la scommessa sarà quella di tenere insieme la specificità delle singole redazioni con la diacronia genealogica dello stemma, e di creare un prodotto aperto ai contributi di orientalisti, storici, storici della lingua, geografi e cartografi.

37 Un prototipo del progetto è stato illustrato da Marina Buzzoni ed Eugenio Burgio nel quinto convegno annuale dell'Associazione per l'Informatica Umanistica e le Culture Digitali (AIUCD), Edizioni digitali: rappresentazione, interoperabilità, analisi del testo e infrastrutture/ Digital Editions: Representation, Interoperability, Text analysis and Infrastructures (Venezia, 7-9 settembre 2016); il progetto ufficiale è stato presentato da Eugenio Burgio, Mario Eusebi e da chi scrive durante la Marco Polo Conference di Tubinga (10 ottobre 2017). 


\title{
«... io essendo giovanetto n'ho udito molte fiate dire...»" Alcune riflessioni sulle fonti dei Viaggi di messer Marco Polo di Giovanni Battista Ramusio
}

\author{
Alvise Andreose \\ (Università degli Studi eCampus, Italia)
}

\begin{abstract}
According to Luigi Foscolo Benedetto's prolegomena to the critical edition of 1928, the Italian translation of Marco Polo's book published by Giovanni Battista Ramusio in the Secondo volume delle Navigazioni e viaggi («R») resulted from the collation of five different texts. One of his main sources was very close to the Latin translation preserved by the manuscript in the Archivio y Biblioteca Capitulares de Toledo, Zelada 49-20 («Z»), which displays supplementary contents of undoubted authenticity compared to the rest of the manuscript transmission. Recent research has proved that the Z-redaction progressively increased in size over the course of time. Some pieces of evidence suggest that Ramusio made his translation from a text containing further authentic variants compared to the model of the Zelada codex. The results reported in the present study confirm the hypothesis that the addenda distinguishing $Z$ and $R$ from all remaining witnesses are the product of Marco Polo's reworking of his book.
\end{abstract}

Sommario 1 La Prefazione sopra il principio del libro del magnifico messer Marco Polo. - 2 Le fonti dei Viaggi di messer Marco Polo. - 3 Il ponte sul «flun de Pulisanghin». - 4 Pulisanghin o Pulisangan?

Keywords Marco Polo. Giovanni Battista Ramusio. Devisement dou monde. Milione. Luigi Foscolo Benedetto. Manoscritto Zelada 49-20. Cina. Pechino. Lugou Qiao. Ponte di Marco Polo.

\section{$1 \quad$ La Prefazione sopra il principio del libro del magnifico messer Marco Polo}

Gli studi che, a partire dall'edizione critica di Luigi Foscolo Benedetto (1928), hanno cercato di fare luce sull'articolato processo elaborativo che è alla base dei Viaggi di messer Marco Polo gentiluomo veneziano pubblicati da Giovanni Battista Ramusio nel Secondo volume delle Navigazioni e viaggi, uscito postumo a Venezia nel $1559,{ }^{1}$ non si sono mai realmente confrontati con l'ipotesi che una parte del materiale incluso in tale versione

1 Sulla traduzione di Ramusio, oltre all'analisi contenuta nei prolegomeni all'edizione critica del testo poliano (Benedetto 1928, CLVIII-CXCIII), si vedano in particolare gli studi inclusi in Burgio 2011 e Simion, Burgio 2015. 
non sia stato ricavato dalla multiforme e complessa tradizione dell'opera poliana, ma derivi da altre fonti, scritte o orali, di cui si è persa traccia. Lo stesso Ramusio, tuttavia, ci fornisce una testimonianza importante - benché problematica - in merito alla circolazione a Venezia di informazioni relative al Devisement dou monde a più di due secoli dalla sua composizione. Nella «Prefazione sopra il principio del libro del magnifico messer Marco Polo», che l'editore premette alla sua traduzione, si riporta un episodio relativo alla stesura del testo che nessun'altra fonte riferisce:

E [Marco Polo, dopo essere stato ferito e catturato dai Genovesi nella battaglia di Curzola] incontinente posto in ferri, fu mandato a Genova, dove, inteso delle sue rare qualità e del maraviglioso viaggio ch'egli avea fatto, concorse tutta la città per vederlo e per parlargli, non avendolo in luogo di prigione, ma come carissimo amico e molto onorato gentiluomo. E gli facevano tanto onore e carezze, che non era mai ora del giorno che dai piú nobili gentiluomini di quella città non fusse visitato, e presentato d'ogni cosa nel vivere necessaria. Or trovandosi in questo stato messer Marco, e vedendo il gran desiderio ch'ognun avea d'intendere le cose del paese del Cataio e del gran Cane, essendo astretto ogni giorno di tornar a riferire con molta fatica, fu consigliato che le dovesse mettere in scrittura: per il qual effetto, tenuto modo che fusse scritto qui a Venezia a suo padre, che dovesse mandargli le sue scritture e memoriali che avea portati seco, e quelli avuti, col mezzo d'un gentiluomo genovese molto suo amico, che si dilettava grandemente di saper le cose del mondo e ogni giorno andava a star seco in prigione per molte ore, scrisse per gratificarlo il presente libro in lingua latina, sí come accostumano li Genovesi in maggior parte fino oggi di scrivere le loro facende, non possendo con la penna esprimere la loro pronuncia naturale. ${ }^{2}$

In un altro punto della Prefazione, Ramusio accenna a informazioni su Marco Polo di cui sarebbe venuto a conoscenza in gioventù:

«... io essendo giovanetto n'ho udito molte fiate dire dal clarissimo messer Gasparo Malipiero, gentiluomo molto vecchio e senatore di singular bontà e integrità ch'avea la sua casa nel canale di S. Marina, e sul cantone ch'è alla bocca del rio di San Giovan Crisostomo, per mezo a punto della ditta corte del Millioni, che riferiva d'averlo inteso ancor lui da suo padre e avo, e d’alcuni altri vecchi uomini suoi vicini...». ${ }^{3}$

\section{Milanesi 1978-88, 3: 31. Cf. anche Ramusio 1559, 7r.}

3 Milanesi 1978-88, 3: 29. Cf. anche Ramusio 1559, 6r. Per un altro dettaglio poliano «fondato sull'akousía di testimoni degni di fede» che Ramusio riferisce nella sezione introduttiva ai Viaggi che va sotto il titolo di «Dichiarazione d'alcuni luoghi ne' libri di messer Marco Polo, con l'istoria del reubarbaro», si veda Burgio 2009, 59-61. 
Benché non tutti i dati contenuti nel passo riprodotto sopra risultino attendibili - falso è, per esempio, il riferimento alla stesura dell'opera in latino, che sappiamo invece essere stata redatta originariamente in francoitaliano $^{4}$-, l'ipotesi che notizie autentiche o comunque veritiere sulla Cina e sull'Estremo Oriente si siano propagate indipendentemente dal mezzo scritto, oppure siano state trasmesse in settori marginali della tradizione che possiamo solo in minima parte ricostruire, non può essere scartata a priori e anzi si ripropone in quei passi dei Viaggi di messer Marco Polo che non trovano riscontro in nessun'altra testimonianza del Devisement dou monde.

\section{Le fonti dei Viaggi di messer Marco Polo}

È noto che, nella complessa vicenda testuale dell'opera, la traduzione ramusiana - che, nel sistema di sigle impostosi a partire dall'edizione di Benedetto, ${ }^{5}$ viene comunemente indicata con la lettera «R» - occupa una posizione di assoluto rilievo. Una delle sue fonti principali era un testimone del Liber de conditionibus et consuetudinibus orientalium regionum (siglato «P»), ${ }^{6}$ cioè della versione latina realizzata dal domenicano Francesco Pipino da Bologna dopo il 1310 e prima del $1322^{7}$ sulla base di un esemplare della famiglia nord-italiana «VA» - tradizionalmente, ma impropriamente, definita «veneta» (Andreose 2002). Tale gruppo di testimoni, insieme ad altre importanti famiglie di codici (F, FG/Fr, TA), ${ }^{8}$ appartiene a quel ramo della tradizione - denominato $\langle A\rangle^{9}$ - che, secondo la ricostruzione bene-

4 Sulla collaborazione tra Marco Polo e Rustichello da Pisa nella stesura del Devisement dou monde - compiuta nelle carceri di Genova nel 1298 -, si vedano in particolare Gossen 1975; Bertolucci Pizzorusso 2011a, 2011b; Rieger 1992; Barbieri 2004; Segre 2008; Andreose $2015 b$.

5 Benedetto 1928, IX-CCXXI. Cf. anche Benedetto 1932, IX-XXIV.

6 Benedetto 1928, CLI: «non deve dimenticarsi che il Marco Polo ramusiano è innanzi tutto una versione di Pipino».

7 Sulla versione di Pipino si vedano, oltre alle fondamentali pagine dell'«Introduzione» di Benedetto (1928, CXXXI- CLVII), i contributi di Dutschke 1993; Gadrat-Ouerfelli 2015, 63-94; Ménard 2017. Il testo, trasmesso da una sessantina di testimoni (Gadrat-Ouerfelli 2015, 91-94, 384-5), attende ancora un'edizione critica.

8 Con «F» Benedetto indica la versione franco-italiana conservata dal ms. BnF fr. 1116; con «FG» la riscrittura 'in buon francese' realizzata in Francia attorno al 1310-1311, con «TA» la versione toscana eseguita all'inizio del Trecento (Benedetto 1928, XI-XCIX). Negli studi recenti, si preferisce indicare la versione francese con «Fr», secondo una consuetudine introdotta da Philippe Ménard (2001-09).

9 L'ipotesi che i testimoni del libro di Marco Polo si distribuiscano in due grandi famiglie non è enunciata in modo esplicito nei prolegomeni dell'edizione del 1928. Benedetto parla per 
dettiana, trasmetterebbe una forma testuale ridotta rispetto all'originale. ${ }^{10}$ $\mathrm{R}$ si attiene alla versione di Pipino nella «squadratura» (Benedetto 1928, CLXII) complessiva della materia, che, come nella fonte latina, viene suddivisa in tre libri, a loro volta articolati in un numero omogeneo di capitoli. ${ }^{11}$ Per ciò che concerne invece la sostanza del testo, la lezione di $\mathrm{P}$ appare sistematicamente 'contaminata' con quella di altri testimoni. Gli studi condotti sulla versione di Ramusio hanno dimostrato che essa è il risultato della collazione di almeno cinque esemplari del Devisement, ciascuno dei quali appartenenti a una diversa famiglia. ${ }^{\mathbf{1 2}}$ Oltre che del Liber pipiniano, l'umanista si sarebbe avvalso del compendio latino «L» (ante 1372), ${ }^{13}$ della versione veneziana «VB» (sec. XIV) ${ }^{\mathbf{1 4}}$ e - specialmente - di un testimone della redazione latina Z (ante 1340). ${ }^{15}$ Meno sicuro è invece l'apporto al testo di $\mathrm{R}$ di un esemplare della versione veneziana «V» (sec. XV). ${ }^{16}$ Secondo la classificazione di Benedetto, L V Z apparterrebbero al gruppo «B», ${ }^{17}$ il cui capostipite sarebbe stato latore di un testo più autorevole e più esteso di quello tràdito dal ramo A. ${ }^{18}$ Particolarmente importante appare, a tale proposito, la traduzione latina $\mathrm{Z}$, che trasmette una serie di informazioni

la prima volta dei due rami «A» e «B» in un saggio uscito a qualche mese di distanza dalla pubblicazione del testo critico, in cui replica duramente alla recensione negativa di Giulio Bertoni (Benedetto 1929, 18, 23, 59; Bertoni 1928; devo questa precisazione alla gentilezza di Samuela Simion). L'idea della bipartizione della tradizione - a cui fa già riferimento la recensione, di tutt'altro tenore, di Mario Casella (1929, 200 nota 1) - sarà ripresa poi da Benedetto nelle pagine introduttive alla traduzione critica del Devisement apparsa quattro anni dopo (Benedetto 1932, XXI-XXII) e in altri saggi successivi (cf. per. es. Benedetto 1959-60, 8).

10 Benedetto 1932, XXI-XXII; cf. anche 1928, CXCVIII-CC.

11 Benedetto 1928, CLXII, CLXXXVIII; Burgio 2011, XVII-XXII (par. di Samuela Simion).

12 Benedetto 1928, CLVIII-CXCIII; Burgio 2011, XIV-XXXVI; Andreose 2015a.

13 Benedetto 1928, CLXXVIII-CLXXXI; Burgio 2011, XXIX-XXXIII (par. di Alvise Andreose).

14 Benedetto 1928, CLXXXII-CLXXXVII; Burgio 2011, XXXIII-XXXVI (par. di Alvise Andreose). Sulla possibilità di retrodatare la versione VB, tràdita da tre testimoni quattrocenteschi, al secolo XIV si veda Simion, in corso di stampa (a).

15 Benedetto 1928, CLXIII-CLXXIII; Barbieri 2004, 48-67; Burgio 2011, XXII-XXV (par. di Giuseppe Mascherpa).

16 Burgio 2011, XXVI-XXIX (par. di Pamela Gennari ed Eugenio Burgio). Su V si veda anche l'analisi di Simion 2011.

17 Sull'origine di tale denominazione si veda quanto detto sopra nella nota 9. Nell'«Introduzione» all'edizione del '28 si parla più genericamente di «Fase anteriore a $F$ » $(B e-$ nedetto 1928, CLVIII-CC). Con il cosiddetto ramo «B» della tradizione potrebbe avere avuto sporadici contatti anche VB, che dipende essenzialmente da un esemplare franco-italiano affine a F, ma presenta isolate lezioni in comune con Z (Benedetto 1928, CLXXXII-CLXXXVII).

18 Sulle caratteristiche di questo gruppo cf. Benedetto 1928, CLVIII-CC. Si veda anche la sintesi di Gadrat-Ouerfelli 2015, 95-111. 
che non figurano in nessun'altra famiglia della tradizione poliana. ${ }^{19}$ Purtroppo, l'unico testimone diretto che ci sia pervenuto, ossia il ms. 49.20 dell'Archivio y Biblioteca capitulares di Toledo, già proprietà del cardinale Francisco Xavier de Zelada (1717-1801), reca un testo in molti punti compendioso. Ramusio, che nella citata «Prefazione» racconta di avere avuto in prestito «da un gentilhuomo» della famiglia patrizia veneziana dei Ghisi «[u]na copia di tal libro, scritta la prima volta latinamente, di maravigliosa antichità, e forse copiata dallo originale di mano di esso messer Marco ${ }^{20}$ e di averla collazionata con un testimone della traduzione di Pipino, ${ }^{21}$ ebbe sicuramente tra le mani un esemplare del gruppo $\mathrm{Z}$ più corretto e completo del codice Zelada (Benedetto1939) - secondo una consuetudine diffusa, indicherò il primo con la sigla $\left\langle\mathrm{Z}^{1}{ }{ }^{22}\right.$ il secondo con $\left\langle\mathrm{Z}^{\mathrm{t}}{ }^{\mathrm{t}}{ }^{23} \mathrm{Ne}\right.$ consegue che la testimonianza di $\mathrm{R}$ risulta di decisiva importanza per ricostruire la fisionomia originaria di tale autorevole famiglia (Benedetto 1928, CLXXIII).

Mette conto rilevare che, a pochi anni di distanza dall'edizione critica del 1928, Benvenuto Terracini ritornò sul problema dei rapporti tra le principali famiglie del resoconto poliano, formulando l'ipotesi - per alcuni versi già implicita nel ragionamento di Benedetto ${ }^{24}$ - che l'antigrafo di $\mathrm{Z}^{\mathrm{t}} \mathrm{e}$ di $\mathrm{R}$, $\mathrm{Z}^{1}$, fosse da collocare in un ramo isolato della tradizione per il fatto che conservava materiali originali che mancavano sia nella famiglia A, sia nelle versioni L e V (Terracini 1933, 398-411). Tale idea si fondava sull'assunto che il testo avesse subito un processo di progressivo impoverimento, che avrebbe implicato, ad ogni snodo della trasmissione, una riduzione graduale dei contenuti originari. ${ }^{25}$ Le indagini più recenti sulla storia testuale del Devisement dou monde, tuttavia, sono venute corroborando l'ipotesi

19 Sulla fisionomia generale della redazione Z, cf. Benedetto 1928, CLXIII-CLXXIII; 195960; Barbieri 1998, 565-78; Mascherpa 2007-08. Cf. anche Gadrat-Ouerfelli 2015, 96-102.

20 Milanesi 1978-88, 3: 32 nota 1. Cf. anche Ramusio 1559, 7r.

21 Ritengo, con Benedetto (1928, CLIX), che Ramusio, laddove parla della copia con cui avrebbe collazionato il codice Ghisi («molte volte ho veduta et incontrata con questa, che al presente mandiamo in luce»), si riferisca al testo del domenicano bolognese, che, almeno in un primo momento, aveva assunto come testo base per la sua edizione (Andreose 2015a).

22 Benedetto 1928, CLXVIII ss. Cf. anche Terracini 1933, 377 ss.

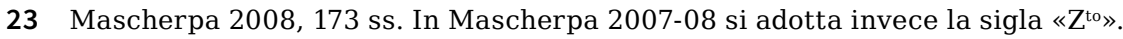

24 Benedetto 1928, CLXXXII, CC. L'idea che i materiali trasmessi esclusivamente da $Z^{t}$ e da R appartenessero alla versione originale (ivi, CXCIX), viene accolta in toto da Casella 1929 e da Peretti 1930.

25 Per Terracini 1933, 398-417, la riduzione sarebbe avvenuta almeno in due fasi: in « $\mathrm{X}^{2} »$, il supposto antigrafo di L V e del capostipite A; e in A, da cui discenderebbero VB, F e gli esemplari franco-italiani da cui dipendono FG, TA, VA. Ricordo che, prendendo le mosse dalle conclusioni di Terracini, Burgio e Eusebi 2008 hanno elaborato una nuova proposta di classificazione, secondo cui Z sarebbe l'unico rappresentante del ramo « $\beta$ », mentre V, L e VB si collocherebbero in vari punti del ramo « $\alpha »$ : V discenderebbe direttamente da $\alpha$, insieme 
alternativa, a più riprese suggerita in passato, ${ }^{26}$ secondo cui un ramo della tradizione trasmetterebbe una prima redazione dell'opera, più sintetica, mentre l'altro - formato dalla famiglia $\mathrm{Z}$ e da $\mathrm{V}$ - ne rifletterebbe uno stadio più recente, risultato di un processo di revisione e integrazione del testo compiuto dal viaggiatore dopo il suo ritorno a Venezia. ${ }^{27}$ Ciò su cui gli studiosi concordano è che la versione che sta a monte di $\mathrm{Z}^{\mathrm{t}}$ e $\mathrm{Z}^{1}$, qualunque sia la sua origine, rispecchi l'ultima volontà autoriale e dunque debba essere assunta come base per un'edizione che voglia restituire nella sua integralità il materiale raccolto da Marco Polo durante il suo soggiorno in Oriente.

\section{Il ponte sul «flun de Pulisanghin»}

I progressi compiuti dalla filologia poliana in tempi recenti ${ }^{28}$ ci mettono nelle condizioni di identificare, con un buon margine di probabilità, le lezioni della traduzione ramusiana che dipendono da $\mathrm{L}, \mathrm{P}, \mathrm{V}$ e VB, e quelle che sono state attinte invece da un affine di $\mathrm{Z}^{\mathrm{t}}$ - per comodità, indicherò d'ora in poi queste ultime con $\left\langle\mathrm{R}^{\mathrm{z}}\right\rangle$. Certo, laddove la testimonianza del toledano manca o è fortemente compendiata, non si può avere l'assoluta certezza che i materiali trasmessi esclusivamente da $\mathrm{R}$ siano autentici. Prima di affermare che tali informazioni risalgono a $Z^{1}$, è necessario vagliare con grande attenzione e prudenza la loro coerenza e la loro attendibilità. Come si può evincere dall'analisi del capitolo dedicato al ponte sul fiume Pulisanghin che presenterò qui di seguito, si tratta in realtà di un'operazione delicata e complessa, che non sempre conduce a risultati definitivi.

Il capitolo ventisettesimo del Libro secondo dei Viaggi di messer Marco Polo - come ho avuto modo di mostrare diffusamente in altra sede (ANdreose 2015d) - è caratterizzato da una cospicua serie di lezioni che lo isolano dal resto della tradizione:

Del fiume Pulisangan et ponte sopra quello. Cap. 27.

Poi che s'è compiuto di dir li governi et administrationi della provincia del Cataio et della città di Cambalú, et della magnificenza del Gran Can, si

al collaterale «ర», formato da FG/Fr, F, L, TA, VA, VB; L costituirebbe con F il gruppo « $\delta^{\text {" } » ; ~}$ e VB farebbe parte del gruppo « $\delta$ '"'», insieme a TA e VA.

26 Barbieri 2004, 145-47; Burgio, Eusebi 2008, 22-23; Burgio 2017. Ė interessante che Benedetto 1928, CXCIX, giudicasse tale ipotesi «né necessaria né utile», benché «astrattamente più che legittima».

27 Si veda il saggio di Samuela Simion in questo volume, che riprende e sviluppa l'ipotesi stemmatica di Burgio, Eusebi 2008, 26-45.

28 Per esempio Burgio 2011; Simion, Burgio 2015. 
dirà delle altre regioni nelle qual messer Marco andò per le occorrentie dell'imperio del Gran Can. Come si parte dalla città di Cambalú et che si ha camminato dieci miglia, si trova un fiume nominato Pulisangan, il quale entra nel mare Oceano, per il qual passano molte navi con grandissime mercantie. Sopra detto fiume è un ponte di pietra molto bello, et forse in tutto il mondo non ve n'è un altro simile. La sua lunghezza è trecento passa et la larghezza otto, di modo che per quello potriano commodamente cavalcare dieci huomini l'uno a lato all'altro. Ha ventiquattro archi et venticinque pile in acqua che li sostengono, et è tutto di pietra serpentina, fatto con grande artificio. Dall'una all'altra banda del ponte è un bel poggio di tavole di marmo et di colonne maestrevolmente ordinate, et nell'ascendere è alquanto piú largo che nella fine dell'ascesa, ma, poi che s'è asceso, trovasi uguale per lungo come se fosse tirato per linea. Et in capo dell'ascesa del ponte è una grandissima colonna et alta, posta sopra una testuggine di marmo; appresso il piè della colonna è un gran leone, et sopra la colonna ve n'è un altro. Verso l'ascesa del ponte è un'altra colonna molto bella, con un leone, discosta dalla prima per un passo et mezzo; et dall'una colonna all'altra è serrato di tavole di marmo, tutte lavorate a diverse scolture et incastrate nelle colonne da lí per lungo del ponte infino al fine. Ciascadune colonne sono distanti l'una dall'altra per un passo et mezzo, et a ciascuna è sopraposto un leone, con tavole di marmo incastratevi dall'una all'altra, accioché non possino cadere coloro che passano: il che è bellissima cosa da vedere. Et nella discesa del ponte è come nell'ascesa. ${ }^{29}$

La maggior parte del capitolo è dedicata alla descrizione di uno tra i monumenti più celebri della Cina imperiale, il ponte di Lugou (Lugou Qiao, 卢沟 桥), detto più comunemente «Ponte di Marco Polo», che oltrepassa il fiume Yongding a sud-ovest di Pechino, a una dozzina di chilometri dall'antica cinta muraria della città (Bretschneider 1876, 50). Benché in tutto il passo riportato si possano individuare differenze più o meno significative rispetto agli altri testimoni del Devisement, la parte in cui emergono le varianti più macroscopiche è quella finale, come si può evincere dalla tabella seguente, in cui - a titolo esemplificativo - si accosta il testo ramusiano a quello di $\mathrm{F}$ (che si assume come esemplificativo di tutta la restante tradizione): ${ }^{30}$

29 Cito il testo secondo l'edizione di Simion 2015b (Libro Secondo: cap. 27). Cf. anche Ramusio 1559, 32v; Milanesi 1978-88, 3: 194-5.

30 Il testo delle principali famiglie del Devisement (FG/Fr, L, TA, V, VA, VB) si può leggere in Andreose 2015d, 130-1 nota 21 (cf. anche Andreose, in corso di stampa). Come si dirà più avanti, in Z questa sezione del capitolo è assente (cf. Barbieri 1998, 116, cap. 46). 


\begin{tabular}{ll}
\hline F CIV $\mathbf{4}$ (et ceteri) $^{\mathbf{3 1}}$ & R II $\mathbf{2 7} \mathbf{7 - 9} \mathbf{9}^{\mathbf{3 2}}$ \\
\hline ...il est fichés en chief dou pont une colone de & Et in capo dell'ascesa del ponte è una \\
marbre et desor la colone a un lion de marbre & grandissima colonna et alta, posta sopra una \\
et desus la colone en a un autre mout biaus et & testuggine di marmo; appresso il piè della \\
grant et bien fait, et longe de cest colone un & colonna è un gran leone, et sopra la colonna ve \\
pas et mi en a un autre toute ausi fait con deus & n'è un altro. Verso l'ascesa del ponte è un'altra \\
lions, et de le une colone a l'autre est clous & colonna molto bella, con un leone, discosta \\
de table de marbre bis por ce que les jens ne & dalla prima per un passo et mezzo; et dall'una \\
peussent cheoir en l'aive... & colonna all'altra è serrato di tavole di marmo, \\
& $\begin{array}{l}\text { tutte lavorate a diverse scolture et incastrate } \\
\text { nelle colonne da lí per lungo del ponte infino }\end{array}$ \\
& $\begin{array}{l}\text { al fine. Ciascadune colonne sono distanti l'una } \\
\text { dall'altra per un passo et mezzo, et a ciascuna } \\
\text { è sopraposto un leone, con tavole di marmo }\end{array}$ \\
& incastratevi dall'una all'altra, accioché non \\
possino cadere coloro che passano...
\end{tabular}

Il confronto sistematico tra le due redazioni ha permesso di dimostrare che entrambe contengono elementi peculiari di sicura o probabile autenticità, $\mathrm{ma}$, al tempo stesso, presentano varie difficoltà interpretative..$^{33}$ Vorrei concentrare qui la mia attenzione su due varianti esclusive di R. L'elemento senz'altro più evidente che distingue il testo ramusiano da quello del resto della tradizione è rappresentato dal riferimento alla «grandissima colonna et alta posta sopra una testuggine di marmo» collocata «in capo dell'ascesa del ponte». Tale dettaglio trova riscontro nell'assetto attuale della costruzione, nonostante la cospicua serie di restauri e rifacimenti realizzati nei secoli successivi al viaggio di Marco Polo in Cina, in epoca Ming e Qing. A ciascuna delle estremità del Lugou Qiao si incontra oggi una statua raffigurante una creatura fantastica tipica della mitologia cinese, il Bixi (员局) - uno dei nove figli del Re Dragone, rappresentato tradizionalmente come un drago ricoperto da un robusto carapace. Si tratta in realtà di manufatti relativamente recenti, che servono da basamento a iscrizioni successive ai lavori di rinnovamento voluti dagli imperatori Kangxi (1661-1722) e Qianlong (17351796). La testimonianza ramusiana, tuttavia, ci suggerisce che monumenti analoghi fossero già presenti in una fase più antica. ${ }^{34}$

31 Eusebi [2010] 2018, 1: 126.

32 Simion, Burgio 2015.

33 Andreose 2015d, 131-6; Andreose, in corso di stampa.

34 Andrà rilevato, tuttavia, che in un dipinto anonimo risalente alla metà del secolo XV (卢沟运筏图, Zattere presso il Lugou Qiao), oggi conservato presso il National Museum of China (中国国家博物馆) di Pechino, i due bixi non compaiono, a differenza di altri dettagli che 
Poco oltre, il testo di R afferma che la colonna poggiante sulla testuggine termina con la statua di un leone («sopra la colonna ve n'è un altro»). Oggi, però, sulla sommità delle stele si trova un bassorilievo raffigurante dei draghi intrecciati, avvolti da una nube. Non si può escludere che la descrizione ramusiana faccia riferimento a un elemento decorativo presente in età Yuan, ma poi modificato in epoca Qing, oppure che l'incongruenza dipenda da un lapsus memoriae di Marco. Mi sembra preferibile, tuttavia, ipotizzare che il sintagma «sopra la colonna» - analogamente al precedente «appresso il piè della colonna» - si riferisca non a una delle due stele che si ergono sopra i bixi, come suggerisce il testo di $\mathrm{R}, \mathrm{ma}$ al primo dei pilastri della balaustra del ponte (anch'esso sormontato da un leone di marmo), ai cui piedi si trovava in età medievale ${ }^{35}$ - e si trova ancor oggi ${ }^{36}$ - la scultura di un leone. L'uso inappropriato dell'articolo definito («sopra la»), che rinvia anaforicamente al referente introdotto immediatamente prima nel discorso, potrebbe essere il riflesso di un'errata interpretazione della fonte latina, che forse leggeva supra columnam. Ma se si confronta con maggiore attenzione la redazione di F con quella di R, si ha la netta impressione che in quest'ultima le pericopi 7 e 8 siano il risultato di un processo di ampliamento della versione originaria, in cui però gli elementi aggiuntivi non sono stati inseriti nel punto esatto del testo. La descrizione di $\mathrm{F}$, benché meno ricca, appare senz'altro più coerente $\mathrm{e}$ perspicua di quella offerta da $\mathrm{R}$.

Il quesito che sorge naturalmente davanti a questa ipotesi è se il materiale supplementare che appare peculiare dei Viaggi di messer Marco Polo risalga a quell'esemplare della famiglia $\mathrm{Z}$ - il codice Ghisi menzionato nella Prefazione - che Ramusio impiegò abbondantemente nella costruzione del testo. Purtroppo, il manoscritto di Toledo omette tutta questa parte del capitolo, ${ }^{37}$ per cui non ci è dato sapere se il suo antigrafo contenesse o meno quella serie di informazioni che isolano $\mathrm{R}$ dal resto della tradizione. Fortunatamente, un aiuto insperato ci giunge da una fonte indiretta, la celebre mappamundi realizzata verso il 1450 da Fra Mauro, camaldolese del monastero dell'Isola di San Michele a Venezia, che oggi si conserva presso

trovano riscontro sia nella descrizione poliana, sia nella forma attuale del ponte. Cf. Huang 2011; Andreose, in corso di stampa.

35 Il dettaglio è raffigurato, per esempio, nel citato dipinto Zattere presso il Lugou Qiao, cf. Andreose, in corso di stampa.

36 In realtà, solo all'estremità orientale del ponte: sul versante occidentale il primo pilastrino è addossato alla scultura di un elefante.

37 Z 46 1-3: «Quando vero discedendo a civitate predicta, scilicet Cambalu, itum est miliaribus .x., invenitur quidam fluvius nomine Pulisanghyn, qui in Occeano Mare intrat. Supra flumen istud est quidam pons lapideus valde pulcer, et raro quod sit in mundo alius ita pulcer. Longitudo eius est trecentorum passuum, latitudo vero .viij., ita quod large posent per eum homines unus a latere alterius equitare, etcetera» (Barbieri 1998, 116). 


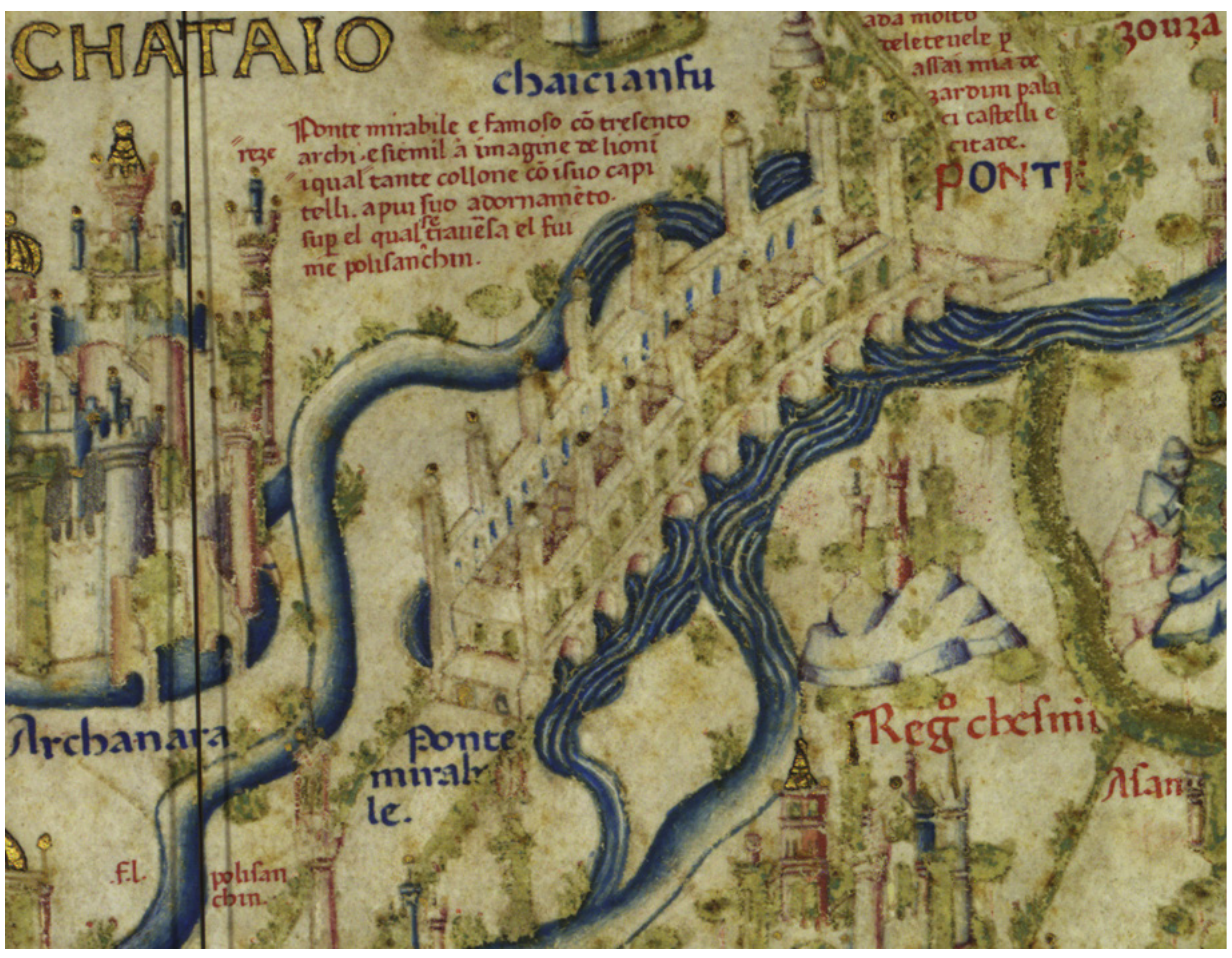

Figura 1. Mappamondo di Fra Mauro, dettaglio (Venezia, Biblioteca Nazionale Marciana, Ridotti dei Procuratori di San Marco)

la Biblioteca Nazionale Marciana. ${ }^{38}$ Come vari studi hanno evidenziato, ${ }^{39}$ per la rappresentazione delle regioni dell'Asia - soprattutto orientale - il cartografo veneziano si basò su un testimone della redazione $\mathrm{Z}$ molto prossimo per lezione al codice Zelada. Ora, a giudicare dall'immagine con cui nel mappamondo viene rappresentato il ponte «mirabile e famoso [...] super el qual se traversa el fiume polisanchin», ${ }^{40}$ parrebbe che la fonte di Fra Mauro non contenesse quel riferimento alla «grandissima colonna et

38 Zurla 1806; Gasparrini Leporace 1956; Cattaneo 2003, 2011; Falchetta 2006.

39 Sui materiali poliani inseriti nel Mappamondo si vedano in particolare Cattaneo 200405, 177-90; 2011, 201-9; Falchetta 2006, 64-7; Burgio 2009, 84-94; Gadrat-Ouerfelli 2015, 101, 228-30.

40 Falchetta 2006, $599 \mathrm{nr}$. 2315: «Ponte mirabile e famoso con tresento archi e siemil à imagine de lioni i qual reze tante collone con i suo capitelli a più adornamento, super el qual se traversa el fiume polisanchin». 
alta, posta sopra una testuggine di marmo» che si legge nella sola versione di R (si veda la fig. 1).

È d'uopo ricordare che Fra Mauro si servì verosimilmente anche di altre fonti poliane ${ }^{41}$ per cui non si può escludere l'eventualità - non impossibile, benché altamente improbabile - che in questo passo abbia seguito un modello diverso da Z. ${ }^{42}$

\section{$4 \quad$ Pulisanghin o Pulisangan?}

L'ipotesi che l'esemplare utilizzato da Ramusio presentasse una fisionomia in parte diversa da quella che possiamo ricostruire attraverso la collazione di tutti i testimoni diretti e indiretti di Z ( $Z$ toledano, il mappamondo di Fra Mauro e altri di cui si dirà) pare trovare conferma in un altro punto del capitolo. Oltre a quella esaminata nei paragrafi precedenti, si dà una seconda variante significativa che isola $\mathrm{R}$ dal resto della tradizione:

\begin{tabular}{|c|c|}
\hline F CIV 3 (et ceteri) ${ }^{43}$ & $R$ II $272^{44}$ \\
\hline $\begin{array}{l}\text { Quant l'en s'en part de la ville et il est alés } \\
. x . \text { miles, adonc trove un grant flum qui est } \\
\text { apellés Pulisanghinz... }\end{array}$ & $\begin{array}{l}\text { Come si parte dalla città di Cambalú et che si } \\
\text { ha camminato dieci miglia, si trova un fiume } \\
\text { nominato Pulisangan... }\end{array}$ \\
\hline
\end{tabular}

Il nome Pulisanghin - che impropriamente Marco Polo attribuisce al corso d'acqua anziché al ponte - riproduce testualmente la locuzione ibrida persiano-cinese Pul-i Sangin, ossia 'Ponte di Sanghin'. La prima parte è persiana, in quanto consta del nome pul (mod. pol) 'ponte', seguito dall'ezāfe -i (mod. -e), elemento enclitico che lega due termini in rapporto di connessione genitivale o aggettivale. ${ }^{45} \mathrm{Il}$ secondo elemento è la

41 Cattaneo 2004-05, 177-90; 2011, 201-9; Burgio 2009; Gadrat-Ouerfelli 2015, 101, 229-30.

42 Questa è l'opinione di Christine Gadrat-Ouerfelli 2015, 230, che fonda il suo ragionamento sul fatto che alcune informazioni riportate nel mappamondo non si trovano nel codice Zelada. Se si considera, però, che vari indizi indicano che lo $\mathrm{Z}$ di cui si servì Fra Mauro era più completo di quello toledano, l'ipotesi che anche la descrizione del ponte sia stata attinta da tale fonte resta quella più verosimile (Burgio 2009, 87-8, 93).

43 Eusebi [2010] 2018, 1: 126.

44 Simion, Burgio 2015.

45 Marsden 1854, 237-8 nota 3; Pauthier 1865, 1: 349-50 nota 2; Yule, Cordier 1903, 2: 5-6; Bretschneider 1876, 54; Hallberg 1906, 415-6; Pelliot 1959-73, 812 nr. 318; Cardona 1975, 703; Haw 2006, 95. Vari interpreti, sulla scorta di Marsden, ritengono che la parte finale della parola corrisponda all'aggettivo persiano sangīn (pers. mod. sangī) 'fatto di pietra, marmoreo', e che dunque la locuzione abbia il significato complessivo di 'Ponte di pietra'. 
traslitterazione persiana del cinese Sanggan (桑干) o, più probabilmente, Sangqian (桑乾), ${ }^{\mathbf{4 6}}$ oronimi un tempo usati per indicare il fiume Yongding, che oggi designano invece il suo corso superiore o uno dei suoi affluenti. ${ }^{47}$ La duplice variante del nome cinese (Sanggan/Sangqian) potrebbe essere all'origine dell'oscillazione che si incontra nella tradizione poliana tra le lezioni Sangan (R) e Sanghin (F et ceteri). Il cinese moderno gān /kân/ e qián (/t $\left.{ }^{\mathrm{h}}{ }_{\mathrm{i}}^{\mathrm{i}} \mathrm{\textrm {n }} /\right)$ corrisponde rispettivamente a /kan/ e /gعn/ del cinese del periodo Yuan, come possiamo dedurre dall'analisi delle traslitterazioni di termini cinesi nell'alfabeto 'Phags-pa (cf. Coblin 2007, 142, 147). Benché la forma oggi prevalente sia Sanggan, il tipo Sangqian era ampiamente diffuso all'epoca della dominazione mongola, sicché è verosimile che, per indicare lo Yongding, i viaggiatori persiani abbiano adottato la seconda variante dell'oronimo (桑乾 - /sang gen/), quasi certamente sovrapponendola al termine persiano sangīn 'fatto di pietra', 'marmoreo' (Cardona 1975, 703). Andrà notato, a tale proposito, che Rashīd al-Dīn chiama questo fiume āb-i Sangīn ossia 'fiume (di) Sangin/Sangqian' ${ }^{48}$ Ciò dimostra che la forma Sangīn era in origine un adattamento del cinese Sangqian - o, meno probabilmente, Sanggan.

Qualunque sia l'origine del toponimo in questione, quel che mi preme sottolineare è che sia la variante Pulisangan tràdita dal testo ramusiano, sia la lezione Pulisanghin che si legge nel resto della tradizione appaiono corrette. La prima si mostra più vicina alla forma cinese del toponimo (Sanggan), la seconda dipende dalla sua traslitterazione - e probabile reinterpretazione - in persiano. Per ritornare al problema della fonte di Ramusio, è estremamente significativo che la lezione Pulisanghin si legga, seppure con minime varianti grafico-fonetiche, anche nel codice Zelada e nel mappamondo di Fra Mauro:

Come si dirà più avanti, è probabile che sia intervenuto un processo paretimologico, che ha fatto sì che l'oronimo cinese Sangqian fosse reinterpretato sulla base della somiglianza con una forma persiana.

46 Si noti che nel cinese di oggi, al carattere 乾 possono corrispondere due forme: gān (/kan/) e qián $\left(/ \mathrm{t}_{6}{ }^{\mathrm{h}}{ }_{\mathrm{i}} \varepsilon \mathrm{n} /\right)$.

47 Pauthier 1865, 1: 349-50 nota 2; Yule, Cordier 1903, 2: 5-6; Bretschneider 1876, 54; Haw 2006, 95; Guy 2010, 202; Ceresa 2015. Il nome Yongding, letteralmente 'sempre stabile', fu adottato all'inizio del secolo XVIII dai Qing con intenzione beneaugurale, dopo che una serie di importanti lavori di idraulica fluviale aveva regolarizzato il corso del fiume. Il nome precedente era Hunhe ossia 'fiume del caos'.

48 Pauthier 1865, 1: 349 nota 2; Bretschneider 1876, 54; Pelliot 1959-73, 812 nr. 318. Cf. Thackston 1998, 2: 441. 
Di fronte a una tale distribuzione delle varianti, che vede $\mathrm{R}$ contrapporsi a tutto il resto della tradizione, ivi compresi $\mathrm{Z}^{\mathrm{t}}$ e Fra Mauro, si possono formulare due ipotesi ricostruttive. La prima è che Ramusio sia venuto in possesso di informazioni vere ma non originali e le abbia inserite nella sua traduzione poliana. Idea che, come si è detto, potrebbe trovare un sostegno nei riferimenti alla circolazione di notizie orali contenuti nella Prefazione ai Viaggi. Un'obiezione seria a tale congettura è che i contatti tra la Cina e l'Europa si erano quasi del tutto interrotti dopo la fine della dominazione mongola sulla Cina e l'avvento della dinastia Ming (1368). I rapporti tra il Celeste Impero e l'Occidente riprenderanno con l'arrivo delle navi portoghesi (1553-57) e, soprattutto, dei gesuiti (1582), eventi contemporanei o posteriori alla stampa ramusiana. Per questa ragione sembra più verosimile una seconda ipotesi, e cioè che Ramusio abbia avuto a disposizione un testimone di $\mathrm{Z}$ che recava delle varianti e delle aggiunte di mano dell'autore che non figuravano nel resto della tradizione, neppure negli esemplari della famiglia $\mathrm{Z}$ da cui dipendono il toledano e il mappamondo di Fra Mauro. L'idea che la redazione Z sia andata progressivamente ampliandosi è stata avanzata con seri argomenti da Giuseppe Mascherpa ${ }^{50}$ e Veronica Gobbato (2015) in due contributi apparsi in anni recenti. Sappiamo, infatti, che la copia ${ }^{51}$ da cui attinsero estratti e citazioni i domenicani Pietro Calò da Chioggia e Filippino (o Filippo) da Ferrara per le loro opere - rispettivamente un leggendario e un manuale di conversazione - attorno al quarto decennio del Trecento ${ }^{52}$ recava una versione di $\mathrm{Z}$ più prossima per lezione a $\mathrm{F}$ e a tutta la restante tradizione (FG, VA, $\mathrm{TA} ; \mathrm{L}, \mathrm{V}, \mathrm{VB}$ ) di quanto non siano $\mathrm{R}^{\mathrm{z}} \mathrm{e} \mathrm{Z}^{\mathrm{t}}$. L'antigrafo di questi ultimi - o più probabilmente delle loro fonti - conteneva delle varianti caratteristiche, ma anche delle significative integrazioni rispetto al modello di Calò e Filip-

49 Falchetta 2006, 599 nrr. 2315, 2321.

50 Mascherpa 2008. Si veda anche Mascherpa 2007-8, 164-71.

51 È oltremodo verosimile che i due domenicani si siano avvalsi non di esemplari simili, ma della stessa copia, conservata probabilmente presso il convento veneziano dei Santi Giovanni e Paolo (cf. Gadrat 2010, 68-70; Gadrat-Ouerfelli 2015, 180; Gobbato 2015, 356-9).

52 Le Legendae di Pietro Calò furono realizzate tra il 1330-1332 e il 1340 (Poncelet 1910, 31; Devos 1948, 258); Il Liber de introductione loquendi di Filippino venne redatto dopo il 1325 e prima del 1347 (Dutschke 1993, 1228-9). Cf. anche Gadrat-Ouerfelli 2015, 167-73, 179-80. 
pino. ${ }^{53}$ Ora, le lezioni specifiche di R nel capitolo sul «fiume Pulisanghin» che ho analizzato in questa sede parrebbero suggerire che Ramusio abbia avuto accesso a una redazione di $\mathrm{Z}$ che presentava ulteriori correzioni e aggiunte rispetto sia alla fonte del manoscritto Zelada, sia a quella di Fra Mauro, ${ }^{54}$ ascrivibili verosimilmente alla mano stessa dell'autore. Un'ipotesi certo affascinante che, tuttavia, potrà essere giudicata attendibile solo se la minuziosa disamina del testo ramusiano sarà in grado di fornire altri esempi di varianti autentiche che lo contrappongono a $\mathrm{Z}$ toledano e agli altri testimoni del Devisement dou monde. ${ }^{55}$

53 Mascherpa 2008, 174-180; Gobbato 2015, 352-5.

54 Ricostruzione che dunque confuterebbe l'ipotesi avanzata - benché con grande cautela - da Falchetta 2006, 67, secondo cui il modello di Fra Mauro «was the Ghisi codex itself». 55 Una conferma di tale idea viene dal saggio di Giuseppe Mascherpa incluso nel presente volume. 


\title{
Sulla fonte Z del Milione di Ramusio L'enigma di Quinsai
}

\author{
Giuseppe Mascherpa \\ (Università di Verona, Italia)
}

\begin{abstract}
The study of the two chapters of the Devisement dou monde concerning the Chinese city of Quinsai, former capital of the Song Empire and a kind of Far Eastern Venice, is crucial to understand some important dynamics of the tradition of Marco Polo's book. Indeed the synoptic reading of these chapters in the redactions $F, Z$ and $R$ - the last two far richer of information than the first - seems to confirm an old, debated hypothesis: the one according to which the Latin redaction Z and Ramusio's Italian version (mostly deriving from a lost $Z$ manuscript) would show traces of a rewriting of some parts of the book, in which some notes by Marco Polo, not used in the Franco-Italian text drafted in Genoa in 1298, would have been included.
\end{abstract}

Sommario 1 Costellazione Z. - $1.1 \mathrm{Z}$ brevior. - 1.2 La fase $\beta^{\prime \prime}$. - 2 Quinsai. - 2.1 Il testo in $Z^{\mathrm{t}}$. - 2.2 Il testo in R. - 3 Conclusioni.

Keywords Marco Polo. Devisement dou monde. Quinsai. Latin redaction Z. Ramusio's Italian version. Rewriting. Textual variants. Marco Polo's notes.

\section{Costellazione Z}

Nel corso dell'ultimo decennio, nel contesto di una rinnovata attenzione per la questione testuale del Devisement dou monde, alcuni studi hanno contribuito a meglio delineare la complessa fisionomia della redazione latina $\mathrm{Z}$, considerata a buon diritto lo snodo fondamentale della tradizione antica del libro poliano: $Z$ conserva infatti, come è noto, un gran numero di passi (da brevi integrazioni a interi paragrafi e addirittura capitoli, a versioni alternative di segmenti di testo) privi di riscontro nella restante tradizione, ${ }^{1}$ ma quasi tutti preziosi per una più precisa definizione della realtà geo-etnografica, storica e politica dell'Asia di Marco Polo.

La redazione $\mathrm{Z}$ è tramandata da un unico esemplare (Toledo, Archivo y Biblioteca Capitulares, ms. Zelada 49-20: d'ora in avanti $Z^{t}$ ), trascritto in Veneto - Venezia, o terraferma orientale - intorno alla metà del Quat-

1 Tranne che, saltuariamente, nella versione veneziana $\mathrm{V}$ e nell'epitome latina $\mathrm{L}$ (Benedetto 1928, CLXXIII-CLXXXI; Simion 2015c; Simion, Burgio 2015 [«Introduzione», § «La redazione L», a cura di Eugenio Burgio). 
trocento e purtroppo radicalmente abbreviato, soprattutto nella prima parte, grosso modo fino all'inizio del 'libro dell'India'; ${ }^{2}$ collaterale del codice toledano, ma più completo, è il perduto esemplare Z (il cosiddetto 'codice Ghisi', siglato $\mathrm{Z}^{\mathrm{g}}$ ) la cui silhouette è individuabile in filigrana al Milione di Ramusio (R), del quale costituì la fonte principale, se non per la struttura globale, senz'altro per i contenuti (le altre fonti, come è noto, furono la versione latina di Pipino [P], un libero rimaneggiamento di area veneto-lagunare [VB], e in misura molto minore la versione veneziana $\mathrm{V}$ e il compendio latino $\mathrm{L}$ ).

Gli addenda di Z, che nella maggior parte dei casi - è opinione corrente presso gli studiosi del Devisement - nulla osta a ricondurre recto tramite all'autore, o quantomeno alla sua cerchia famigliare, convinsero Luigi Foscolo Benedetto a riservare alla redazione latina una posizione altissima nell'ipotetico stemma del Devisement dou monde; lo studioso vedeva infatti in $\mathrm{Z}$ la traduzione di un testo franco-italiano molto vicino all'originale composto a Genova da Marco Polo e Rustichello da Pisa, la cui successiva, ampia sfrondatura, promossa da esigenze editoriali, avrebbe prodotto l'archetipo della restante tradizione, ${ }^{3}$ di cui il ms. français 1116 della Bibliothèque Nationale di Parigi $(F)$ sarebbe un fedele riflesso.

\subsection{Z brevior}

Questa ricostruzione, anche in ragione dell'autorevolezza di Benedetto e della monumentalità della sua sistemazione critica, ha goduto di ottima salute fino a pochi anni or sono, allorché nuove indagini sulla tradizione indiretta di $\mathrm{Z}$ in due compilazioni trecentesche, riconducibili al milieu domenicano del Veneto meridionale, hanno condotto a un sostanziale ribaltamento di prospettiva. Lo studio degli excerpta poliani contenuti nel Legendarium agiografico di Pietro Calò da Chioggia e nel Liber de introductione loquendi di Filippino da Ferrara ${ }^{4}$ (entrambi ante

2 Rispetto a $F$, Z è privo di una sessantina di capitoli, mentre altri risultano inficiati da tagli più o meno corposi (Benedetto 1928, CLXIV; Barbieri 1998, 576-8; Mascherpa 2007-08, 78-99).

3 Con l'eccezione di V e L, nei quali Benedetto e Terracini individuavano, in ragione dei loro punti di contatto con $\mathrm{Z}$ nel contesto di una sostanziale aderenza a F, l'espressione di una tappa intermedia nel processo di riduzione del Devisement originario (Benedetto 1928, CLXXXII; Terracini 1933, 409-20).

4 Sui brani del Liber de introductione loquendi («particolare raccolta di narrazioni ed exempla ordinati in otto libri, ciascuno dei quali relativo ad una 'circostanza' della vita sociale in cui un frate è chiamato a prendere la parola»: Gobbato 2015, 319-20) che Filippino desume dal Devisement dou monde è imprescindibile lo studio di Gobbato 2015. Quanto al capitolo del Devisement sulla predicazione orientale di San Tommaso Apostolo, incastonato da Pietro Calò nel suo Legendarium, l'edizione del testo è in Devos 1948, l'inquadramento ecdotico in Mascherpa 2008, ripreso in Mascherpa 2007-08, 164-71. 
1350) ha infatti rivelato come gli esemplari Z utilizzati dai compilatori fossero, almeno nei segmenti selezionati, privi degli addenda caratteristici dell'antecedente comune di $\mathrm{Z}^{\mathrm{g}} / \mathrm{Z}^{\mathrm{t}}$ (siglato $\beta^{\prime \prime}$ nel nuovo stemma del Devisement presentato in questo volume) e, se si eccettua qualche minimo incremento testuale, grosso modo coerenti con $\mathrm{F}$ nella forma e nei contenuti. ${ }^{5}$ Si considerino un paio di esempi, selezionati nell'ambito di una casistica molto varia. ${ }^{6}$

(a) Il culto di San Tommaso Apostolo nell'India sud-orientale (Mascherpa 2008)

Nella sezione del Legendarium dedicata a San Tommaso Apostolo, il capitolo di Marco Polo occupa una posizione di rilievo tra le fonti utilizzate da Pietro Calò, in quanto offre al compilatore non solo un resoconto sulla predicazione e il martirio dell'Apostolo nel Maabar e sulla persistenza di un culto locale (interconfessionale) a lui dedicato, ma anche la narrazione di un miracolo post mortem non altrimenti attestato.

Nell'estratto qui riportato emerge come l'esemplare Z utilizzato da Calò rispecchiasse le movenze di $\mathrm{F}$ e della restante tradizione $v s \mathrm{Z}^{\mathrm{t}}$, oltre che nel procedere sintattico, anche nella transizione diretta 2 a 3 senza l'inserto 2a (la coltivazione delle palme da cocco nell'area del santurario), che è proprio dei soli $\mathrm{Z}^{\mathrm{t}}$ e (si veda infra) $\mathrm{R}$.

5 L'appartenenza del Milione di Pietro Calò alla famiglia Z, che Benedetto si limitò a segnalare senza il corredo di una dimostrazione (Benedetto 1959-60, 573-5), è sancita dalla condivisione di un errore congiuntivo con il testo di Toledo: nell'episodio del martirio di San Tommaso, $\mathrm{Z}^{\mathrm{t}} \mathrm{e}$ il testo del Legendarium concordano infatti nell'affermare che il santo muore trafitto in tibiam dexteram $\left(\mathrm{Z}^{\mathfrak{t}}\right)$ /in tybia dextra (Leg.) dalla freccia scagliata da un pagano, laddove il resto della tradizione, più plausibilmente, riferisce invece di una ferita al costato: $\mathrm{F}$ destre costee, L dextrum latus, TA per le costi, V in lo ladi destro, ecc. (Simion 2017a, 26 nota 16). Nessun errore congiuntivo, ma una nutrita schiera di varianti - sia formali che sostanziali - rivela un esemplare Z anche nella fonte poliana di Filippino (Gobbato 2015, 329-55). Sulla possibilità che la versione $\mathrm{V}$ dipenda da uno $\mathrm{Z}$ con le stesse caratteristiche di quelli utilizzati dai domenicani si vedano le osservazioni di Samuela Simion raccolte e commentate in Simion 2011 e, più recentemente, in Simion 2015c. Pare uno Z di questo tipo anche quello utilizzato, probabilmente per contaminazione, nella stesura dell'epitome L.

6 Negli esempi, in carattere sottolineato le lezioni che accomunano tutti i testimoni $Z$ vs $F$, in corsivo quelle riconducibili al solo antecedente comune di $\mathrm{Z}^{\mathrm{t}} / \mathrm{Z}^{\mathrm{g}}\left(\beta^{\prime \prime \prime}\right)$. 


\section{$Z^{\mathrm{t}} 109$ 1-15}

1. Corpus quidem Beati Thome apostoli est in provincia Maabar in quadam civitate parva, in qua sunt pauci mercatores et homines, neque illuc veniunt quia ibi sunt pauca mercimonia que illinc possi<nst extrahi, et etiam locus multum devius est.

2. Bene verum est quod multi christiani et saraceni illuc veniunt propter devotionem: nam saraceni illius contrate habent magnam devotionem in ipso; dicunt enim quod fuit saracenus et quod magnus propheta est, et nuncupant eum avarium, quod est dicere 'sanctus homo'.

\section{2a. Christiani qui ecclesiam custodiu[nt] multas habent arbores, que vinum faciunt et que nuces pharaonis producunt nam de una nuce pasceretur unus homo cibo et potu [...] pro qualibet arborum istarum solvunt christiani uni ex quatuor fratribus regibus in provincia Maabar in quolibet mense groso uno.}
3. Et dicemus vobis de mirabilibus que ibi sunt. Noveritis itaque quod christiani qui illuc propter devotionem accedunt accipiunt de terra ubi fuit mortuus sanctus thomas et illam terram in eorum patriam perportant et dant ad potandum de ista terra pacientibus febres tercianas vel quartanas et statim cum eger potaverit liberatus est. Et hoc accidit omnibus egris potantibus hanc terram; terra quidem rubea est. Et dominus Marcus de hac terra secum portavit Venecijs et multos liberavit cum ipsa.

\section{Legendarium}

1. Est igitur corpus eius in quadam civitate parva in qua sunt pauci mercatores et homines, neque illuc veniunt quia ibi non sunt mercimonia que inde possint extrahi, et est locus multum devius.

2. Multi autem christiani et saraceni illuc veniunt propter devotionem in eum, et dicunt quod fuit saracenus, in hoc mencientes, quia Thomas apostolus iudeus fuit, et nominant eum avarion, id est bonum hominem.

\section{F 175 2-4}

1. Le cors meisser Saint Thomeu le apostres est en la provence de Meabar, en une petite ville; car ne a gueires homes, ne mercaant ne $\mathrm{i}$ vienent por ce que n'i a mercandies que bien en peust traire et encore que le leu est mout desviables.

2. Bien est il voir que maint cristiens et mant saraçin hi vienent en perlinajes: car je voç di que le saraçin de celle contree hi ont grant foi et dient qu'il fui saraçin, et dient qu'el est profit grant et l'apellent avarian, que vaut a dire saint home.

\begin{abstract}
3. Christiani autem quui illuc propter devotionem accedunt, accipiunt de terra ubi mortuus fuit sanctus apostolus, et illam in suam patriam portant, et dant ad potandum de ista terra cuicumque pacienti febres quartanas vel tercianas vel alias. Et statim cum eger potaverit liberatus est. Et hoc accidit omnibus egris potantibus de hac terra, que est rubea. Et dominus Marchus prefatus portavit secum de terra ista Venecias et multos liberavit cum ipsa.
\end{abstract}

\begin{abstract}
3. E si sachiés que il hi a tel mervoie com je vos conterai: or saquiés que les cristienç, que vont la en pelegrinajes, prenne«n`t de la tere dou leu, la ou le saint cors fou mort, e celle terre aportent en le lor contree, e donent de ceste une pou a boir au malaide quant ausse fevre quartaine ou tersaine ou ceste tiel fevre; et tant tost que le malaide l'a beue, el est guaris. Et ce avint a tuit celz amalaides que celle terre boivent, e sachiés que'elle est terre roge.
\end{abstract}

Ramusio desume l'intero episodio da P ma ne integra il dettato con la pericope sulle palme da cocco, che in $\mathrm{Z}^{\mathrm{g}}$ doveva leggersi in una forma molto vicina alla lezione toledana: ${ }^{7}$

7 Ramusio taglia però la descrizione del frutto, già utilizzata nel capitolo su Samara (III 13 8) per integrare la lezione di VB (136 11). 
... Et molti altri miracoli tutto il giorno si veggono, per intercessione di questo beato apostolo. I christiani che custodiscono detta chiesa hanno molti arbori che fanno le noci d'India che habbiamo scritto di sopra, quali li danno il vivere, et pagano ad un di questi re fratelli un grosso ogni mese per arbore. (R III 20 65-6)

(b) Le palme da sago nel regno di Fansur (Gobbato 2015, 352-3)

La digressione 'meravigliosa' sulla coltivazione (a tutt'oggi fiorente) della palma di sago a Sumatra fa parte dell'eterogeneo drappello di tessere poliane incluse nel Liber de introductione loquendi di Filippino da Ferrara. Di nuovo, come nel caso di Pietro Calò, ci si imbatte in un esemplare Z aderente al dettato di $F$ nella forma e nei contenuti: ${ }^{8}$ manca infatti, nella fonte $\mathrm{Z}$ di Filippino, il minuto resoconto sull'estrazione e lavorazione della 'farina' che invece caratterizza $\beta$ '".

\section{$Z^{\mathrm{t}} 103$ 6-13}

...habent enim maneriem unam arborum que sunt multum grose et longe, et earum lignum est circumcirca forte per tres digittos grossum, et tota medula interior est farina, [...] et farina emundata in fundo remanet comprehensa, et tunc conditur et fiunt ex ea lagana et diverse epule que de pasta fiunt, que sunt valde bone, et Dominus Marcus multociens hoc probavit

\section{R III 16 4-6}

...hanno una sorte di arbori grossi et lunghi, alli quali levatali la prima scorza, che è sottile, si trova poi il suo legno grosso intorno intorno per tre dita, et tutta la midolla di dentro è farina come quella del caruol [...] et la farina purgata et mondata che rimane si adopra, et fansi di quella lasagne et diverse vivande di pasta, delle qual ne ha mangiato piú volte il detto messer Marco

\section{Liber I 20 2-4}

...est una generatio arborum que habent corticem subtilem, et sub cortice sunt plene farina, et faciunt multa comestibilia de pasta que sunt valde bona. Et dominus Marcus hoc probavit multociens.

\begin{abstract}
F 1696
...il ont une mainere d'arbres qe mout sont groses e grant. E cesti arbres sunt tuit plein dedans de farine; qe sachiés qe cesti arbres ont mout soutil escorces, e tuit dedens est farine, et ne font meint mengier de paste qe mout sunt buen a mangier, car je voç di qe nos meesme les provammes aseç, car nos en menuiames plusors foies
\end{abstract}

\subsection{La fase $\beta^{\prime \prime \prime}$}

Gli esempi (a) e (b) dimostrano dunque l'esistenza e la circolazione di quello che si potrebbe definire uno ' $\mathrm{Z}$ brevior': ovvero una prima versione latina, messa insieme nella prima metà del Trecento, di un testimone franco-italiano non distante da F ma qualche punto più ricco. Sullo sche-

8 Meno che nello sviluppo «...qe mout sont groses e grant. E cesti arbres sunt tuit plein dedans de farine; qe sachiés qe cesti arbres...», mancante in tutti i testimoni del trattato di Filippino: può trattarsi di lacuna per saut du même au même (su arbores/arborum?) prodottasi nell'archetipo del Liber o già presente nella fonte del domenicano, oppure della semplice sfrondatura di un passo oggettivamente ridondante. 
letro di questa traduzione si sarebbe poi compiuto un ulteriore processo rielaborativo, volto essenzialmente all'espansione (anche considerevole) del testo di partenza, sia tramite la riscrittura approfondita di segmenti testuali già dati, che con l'innesto di brani - descrittivi e narrativi - di dimensioni variabili e completamente nuovi. Prodotto di questa fase redazionale è $\beta^{\prime \prime \prime}$, la cui fisionomia complessiva si può ricavare virtualmente dalla triangolazione tra $Z^{t}, R$ (laddove rifletta $Z^{g}$ ) e F.

Quello che emerge dalla sinossi, in coerenza con le osservazioni di Benedetto, è prima di tutto lo scarto qualitativo che separa $\mathrm{Z}^{\mathrm{g}}$ e $\mathrm{Z}^{\mathrm{t}}$ sotto il profilo dell'integrità testuale in corrispondenza dei primi due libri di $\mathrm{R}$, dove la versione ramusiana consente la ricostruzione della silhouette di $\beta^{\prime \prime \prime}$, emendando la lezione aspramente ridotta o del tutto mancante del Toledano. Con il terzo libro si innesta invece il movimento contrario: è la lezione di $\mathrm{Z}^{\mathrm{t}}$, da un certo punto in avanti sostanzialmente completa, a restituire il testo di $\beta^{\prime \prime \prime}$, mentre Ramusio ricorre più spesso alle altre fonti $\mathrm{e}$, quando utilizza $\mathrm{Z}^{\mathrm{g}}$, non esita a sunteggiarlo, sia nei passi in comune con la tradizione che negli addenda (o forse $\mathrm{Z}^{\mathrm{g}}$, con situazione speculare a quella del Toledano, presentava in questa sezione un testo lacunoso).

In qualche luogo, la sinossi $Z^{\mathrm{t}}-\mathrm{R}-\mathrm{F}$ serve altresì a evidenziare come nella 'fase Z' non abbiano agito soltanto dinamiche espansive, ma anche, contestualmente ad esse, strategie di riorganizzazione testuale (rimescolamento di tessere; contrazione/accorpamento) tipiche di un po' tutta la tradizione antica del Devisement. Emergono in particolare casi di

a. interventi sulla dispositio delle pericopi: ad esempio, nei capitoli dedicati alla pianura tra Qamadin e Hormuz (F XXXV-XXXVI; R I 14-16; Z 13-14), nei quali $\beta$ "' presenta una scaletta alternativa, complessivamente più razionale, del materiale corografico;

b. riduzione del dettato con accorpamento di capitoli e tagli di varia entità: ad esempio nel racconto del miracolo di Baghdad, sfrondato di molti pleonasmi (F XXV-XXVIII; R I 8 11-29; Z 7); nella sezione dedicata al Vecchio della Montagna (F XL-XLII; R I 21; Zt 17), dove vengono eliminate le ridondanze della parte centrale (corrispondente, all'incirca, al segmento di F XLI 6-XLII 7); nel capitolo su Samarcanda (R I 30 4-7; F LI 5-17; Z 26 1), che in $\beta^{\prime \prime \prime}$ mancava probabilmente della narrazione del miracolo di San Giovanni Battista. In tutti e tre i casi - oltre che in molti altri, anche minuti - il fatto che Ramusio ricorra a P o VB nei punti esatti in cui $\mathrm{Z}^{\mathrm{t}}$ si presenta abbreviato o mancante induce a credere che anche $\mathrm{Z}^{\mathrm{g}}$ recasse, nei passi in questione, un testo scorciato. ${ }^{9}$

9 Per ragioni di sintesi, si rimanda ai commenti ad loca contenuti in Simion, Burgio 2015. 
Un ultimo, ma non meno rilevante aspetto sul quale la sinossi invita a riflettere è la questione degli addenda singolari di $\mathrm{R} \mathrm{e} \mathrm{Z}^{\mathrm{t}}$, quelli che, attestati soltanto nell'uno o nell'altro testimone, non sono riconducibili a $\beta$ '"' per via stemmatica. ${ }^{10}$ In molti casi questi ajouts hanno l'aspetto di glosse esegetiche o di note erudite alla portata di qualunque copista o rielaboratore. ${ }^{11}$ Altrove invece si tratta invece di brani genuini nei contenuti (di 'sapore poliano', si potrebbe dire) e talora così lunghi e articolati da costituire unità testuali autonome nel mosaico multiforme di Z. Oltre all'ampia casistica offerta, sia per $\mathrm{Z}^{\mathrm{t}}$ che per R, dal capitolo su Quinsai (cf. infra, § 2), si ricordano, tra i brani singolari di R, il capitolo su un monastero persiano intitolato all'eremita Barsauma (I 10), la descrizione degli effetti devastanti del caldo di Hormuz (I 15 8-10), il breve excursus - certamente genuino ${ }^{12}$ - sui tatuatori dello Yunnan (I 18 4; cf. infra, es. (a)); tra gli ancora più numerosi unica di $\mathrm{Z}^{\mathrm{t}}$, invece, il capitolo corografico sullo Iuguristan (33), l'aneddoto sulle facoltà psicotrope della terra del Kirman (12 4-11: es. (b)), o quello sulle stravaganti pratiche divinatorie dei marinai indiani (91 30-40).

In linea di massima, mancando prove testuali cogenti, non v'è ragione di dubitare che tutti questi addenda fossero effettivamente presenti in $\beta^{\prime \prime \prime}$, e che la loro assenza - a seconda - in $\mathrm{Z}^{\mathrm{t}}$ o $\mathrm{R}$ sia da imputare, per il primo, ai tagli del copista-riduttore, per il secondo, alle scelte editoriali di Ramusio, oppure a una campagna di sfoltimento messa in atto dai rispettivi antecedenti: dinamiche dietro alle quali non è sempre possibile individuare un disegno coerente. Ci si può tuttavia imbattere (a) in casi in cui $\mathrm{R}\left(<\mathrm{Z}^{\mathrm{g}}\right)$ presenta un addendum singolare laddove $Z^{t}$ ricalca nella struttura e nei contenuti il testo della tradizione $\left(\mathrm{Z}^{\mathrm{t}}=\mathrm{F}\right.$ vs $\left.\mathrm{Z}^{\mathrm{g}}\right)$; (b) oppure, specularmente, in tessere singolari di $Z^{\mathrm{t}}$ laddove $\mathrm{R}\left(<\mathrm{Z}^{\mathrm{g}}\right)$ risulta allineato a $\mathrm{F}\left(\mathrm{Z}^{\mathrm{g}}=\mathrm{F}\right.$ vs $\left.\mathrm{Z}^{\mathrm{t}}\right)$.

10 Onde evitare falsi positivi, si circoscrive l'indagine sugli addenda singolari alle sole sezioni in cui $\mathrm{Z}^{\mathrm{t}}$ non sia drasticamente scorciato.

11 Qualche esempio: R I 8: «[il ciabattino] immediate con una delle stecche che adoprava in bottega si cavò l'occhio destro; la qual cosa dimostrò manifestamente la grandezza della sua constante fede»; R III 43 8: «Ha un castello molto forte, che tiene a modo di dir serrato il colfo et il mare, perché discopre tutte le navi da ogni tempo che passano»; $\mathrm{Z}^{\mathrm{t}}$ 9: «diem miraculi huius Christiani Nestorini et Iacopiti solempniter celebrant, continue in vigilia ieiunantes. Nota quod christiani, armeni, nestorini et iacopiti diferunt in certis articulis, ymo ex hoc unus alium repudiat et aborret»; $\mathrm{Z}^{\mathrm{t}} 101$ 10: «Na<m» si substantia aliqua remaneret, illa procrearet vermes; et vermes deficiente esca morirentur; et de obitu istorum vermium dicunt ipsi quod anima defuncti pecatum magnum haberet, videlicet quod tot anime ex substantia sua orte perirent» (cf. le schede di Burgio e Mascherpa ad loca in Simion, Burgio 2015).

12 L'informazione di R aderisce perfettamente al dato etnografico: cf. Yule, Cordier 1903, 2: 128 . 
(a)

\section{R || 41 3-5}

Gli huomini et le donne di questa provincia usano di portare li denti coperti d'una sottil lametta d'oro, fatta molto maestrevolmente a similitudine di denti, che li coprono, et vi sta di continuo

Gli huomini si fanno anchor a torno le braccia et le gambe a modo di una lista o vero cinta, con punti neri, designata in questo modo: hanno cinque agucchie tutte legate insieme, et con quelle si pungono talmente la carne che vi esce il sangue, et poi vi mettono sopra una tintura nera, che mai piú si puol cancellare; et reputano per cosa nobile et bella haver questa tal lista di punti neri.

Et non attendono ad altro se non a cavalcare et andare alla caccia et uccellare...

(b)

\section{$Z^{\mathrm{t}}$ 12-13 1}

Chermam quoddam regnum est in confinibus Persie versus levantem, quod heredes antiquitus succedebant. In isto regno lapides oriuntur nomine turchiese, qui fodiuntur in venis montium; inveniuntur etiam in montibus illis vene calibis et andaniqui in maxima quantitate. In hyeme tam intensum frigos $h$ rabsetur, quod vix homo potest evadere continue portando multas vestes et peles.

\section{$Z^{t} 58$ 3-4}

Gentes ipsius dentes habent deauratos, videlicet quod omnes dentes sunt coperti auro: nam fieri faciunt in similitudinem dentium unam formam de auro, cum qua dentes coperiunt.

Homines itaque, secundum eorum consuetudinem, milites sunt omnes, et ad nil aliud intendunt quam ad aucupationes, venationes...

\section{R I 13-14 1}

Chiermain è un regno ne' confini della Persia verso levante, il qual anticamente andava de herede in herede, ma dopo che 'I Tartaro lo soggiogò al suo dominio non succedettero gli heredi, anzi il Tartaro vi manda signore secondo il voler suo. In detto regno nascono le pietre che si chiamano turchese, quali si cavano nelle vene de' monti; si trovano anchora in quelli vene di azzaio et andanico in grandissima quantità. [...] nel tempo dell'inverno vi è cosí gran freddo, che appena l'huomo si può riparare portando continovamente molte vesti et pelli.

Dapoi la discesa di questo luogo per le dette due giornate si trova una gran pianura

\section{F 119 4-5}

Les jens ont tous les dens d'or, ce est qe chascun dens est covert d'or, car il font une forme d'or faite a la mainere de seç dens et couvrent les dens desout come celz desovre; et ce font les homes et nen les dames.

Et les homes sunt tuit chevalers, selonc lor usance, et ne font rien for qe aler en host et aler chaçant et oselant

\section{F 34-35 2}

Crerman est un rengne en Perse mesme et ansiene (ment) so seingnore l'oit por hereditajes; mes, puis que le Tartar le conquistrent, ne vait pas la segnorie por hereditajes, mes hi mande le Tartar celui sire qu'il vult. En cest regne naisent les pieres que l'en apele torchoise, et hi ni a en grant habundance, car il les trevent en les montagnes, car il le escavent dedens la roche. Et encore ont vene d'acier et d'ondanique assés. [...] ha si grant froit de yver que a poine eschanpe l'en portant aséç dras et aséç pannes.

Et quant l'en ha descendu celle deus jorné que je voç ai dit, adonc treve une grandisme plaingne... 
Gli esempi mostrati sembrano aprire alla possibilità che qualcuna delle aggiunte più significative di $\mathrm{Z}$ sia stata accolta a testo non all'altezza di $\beta^{\prime \prime \prime}$, bensì dei rispettivi antigrafi di $Z^{\mathrm{t}} \mathrm{e} R\left(\mathrm{Z}^{\mathrm{g}}\right)$. Il che rivelerebbe, almeno per alcune parti del libro, una 'fase $Z$ ' testualmente irrequieta e attiva fino ai piani più bassi della sua tradizione, nella quale anche le ultime diramazioni dello stemma sarebbero state singolarmente permeabili all'innesto di materiali nuovi - se non proprio riconducibili all'autore, almeno autentici nei contenuti e nella forma coerenti con l'habitus poliano.

Del resto, numerosi indizi - la presenza di addenda genuini, il marcato radicamento lagunare ${ }^{13} \mathrm{e}$ in particolare la circolazione entro il milieu domenicano della città di Venezia ${ }^{14}$ - suggeriscono che la redazione $\mathrm{Z}$ sia stata effettivamente prodotta in un contesto prossimo alla cerchia familiare dell'autore, e che la sua mouvance ( $\mathrm{Z}$ brevior $>\beta^{\prime \prime \prime}>$ innesti singularia in $\left.\mathrm{Z}^{\mathrm{g}} / \mathrm{Z}^{\mathrm{t}}\right)$ rappresenti in qualche modo il 'processo evolutivo' del testo di Marco Polo.

Le più consistenti pezze d'appoggio a questa ricostruzione risiedono nelle dinamiche testuali che coinvolgono, in $\mathrm{R}$ e $\mathrm{Z}^{\mathrm{t}}$, la monografia sulla città di Quinsai, la cui complessità rende indispensabile una trattazione autonoma.

\section{Quinsai ${ }^{15}$}

Tra XIII e XIV secolo la città di Quinsai (oggi Hang-zhou), antica e splendida capitale dell'impero Song conquistata dai Mongoli nel 1276, veniva celebrata dai viaggiatori occidentali come una delle più straordinarie meraviglie urbane del Levante. Percorsa da una fitta rete di canali e affacciata su un grande lago, Quinsai godeva di un clima mite e di una posizione strategica. La sua particolare topografia, insieme alle vie brulicanti di vita,

13 A quest'area puntano quasi esclusivamente gli indizi offerti dalla geografia della tradizione: $\mathrm{Z}^{\mathrm{t}}$ è localizzabile, sulla base della filigrana, a Venezia o tutt'al più nel Veneto orientale (Mascherpa 2007-08, 17-18); di ambito veneziano sono le redazioni L, V e VB, in varia misura compromesse con la 'fase Z' (Burgio, Mascherpa 2007; Simion, Burgio 2015). Dal convento dei Santi Giovanni e Paolo transitarono - uno come studente, l'altro come priore - Filippino da Ferrara nel 1307 e Pietro Calò nel 1328 (Gobbato 2015, 357); a San Michele di Murano il camaldolese fra Mauro, attorno al 1450, utilizzò un esemplare Z come fonte per la compilazione della sua Mappa mundi (Cattaneo 2005b; Burgio 2009). A Venezia e su materiali principalmente veneziani lavora, infine, Ramusio.

14 Sul rapporto tra Marco Polo e i domenicani del convento dei SS. Giovanni e Paolo di Venezia si veda ora Gobbato 2015, 357-60.

15 In questo paragrafo rifondo parte dei contenuti di un mio recente contributo, in corso di stampa negli atti del convegno internazionale Predicatori, mercanti, pellegrini. L'Occidente medievale e lo sguardo letterario sull'Altro tra l'Europa e il Levante (Sassari, 11-12 maggio 2016). 
alle piazze invase dai mercati e ai palazzi signorili, deve avere restituito agli occhi di Marco Polo l'immagine di una splendida Venezia d'Oriente, la cui descrizione si sviluppa nell'ampia e articolata monografia contenuta nei capitoli 151 e 152 di F. ${ }^{16}$ La sostanza testuale e l'ordine espositivo della redazione franco-italiana sono ribaditi senza variazioni degne di nota in quasi tutti i testimoni della tradizione antica, ${ }^{17}$ meno che in $\mathrm{Z}^{\mathrm{t}} \mathrm{e}$ - soprattutto - in R, la cui versione del trattato sulla 'città del cielo' (e in particolare del primo dei due capitoli ad essa dedicati) risulta profondamente rivoluzionata. ${ }^{18}$

\subsection{Il testo in $\mathrm{Z}^{\mathrm{t}}$}

Nel codice Toledano i capitoli su Quinsai (85-86) presentano una dispositio degli argomenti sostanzialmente identica a quella di F, fatta eccezione per l'inversione di due excursus etnografici adiacenti dedicati alle pratiche astrologiche e alle consuetudini funebri del Mangi (cf. tav. 1). Differenze sostanziose si notano invece sul piano dei contenuti, dove, al netto dei tagli prodotti dall'azione indiscriminata del riduttore - sia nel corpo del capitolo che in fine, dove un etcetera tronca bruscamente il flusso testuale $^{19}$ - il testo toledano risulta in più punti arricchito dall'inserzione di ajouts di dimensioni variabili del tutto sconosciuti alla restante tradizione, tranne che, in qualche caso, a R (che ne condivide alcuni ma non altri): si tratta di aggiunte brevi innestate su porzioni testuali già date, allo scopo di precisarle (a1) e, nel caso, rettificarle (a2), o di brani nuovi molto estesi e del tutto autonomi rispetto al testo della tradizione (es. [b1], [b2]), principalmente di carattere etnografico e storico, con digressioni legate alla conquista mongola della città, di cui Marco Polo fu testimone diretto.

16 F 151 (rubr.): «Ci devise de la noble cité de Quinsai»; F 152 (rubr.): «Ci devise de la grant rende que le Gran Kaan a de Qinsay».

17 Quanto all'ordine degli argomenti, le innovazioni più interessanti si riscontrano nel 'Milione veneto' VA (114): si tratta però, con ogni probabilità, di interventi di ristrutturazione operati da un rimaneggiatore ai piani bassi dello stemma, giacché il testo di Pipino, dipendente da un esemplare VA più vicino all'archetipo, presenta la stessa struttura di F. Alcune singolarità strutturali occorrono anche in VB 114, che in un luogo del testo presenta addirittura un addendum senza riscontri nella tradizione (cf. Gennari 2008-09, 161, § 17 e la nota di A. Andreose a R II 6863 in Simion, Burgio 2015). Mancano infine alcune pericopi in L, V, per il resto sostanzialmente allineati a $\mathrm{F}$.

18 Struttura e contenuti del capitolo sono riassunti nelle tavole sinottiche (F-Z; F-R) poste in appendice.

19 Gli etcetera di $\mathrm{Z}^{\mathrm{t}}$ troncano pericope e capitolo anche nei capp. 31, 45-7, 79, 100; interrompono pericopi interne nei capp. 59 e 90. 
(a1) Il materiale dei ponti ${ }^{20}$

\section{F 1517}

Il se contenoit tuit primermant ‘ques la cité de Quinsai gir'environ .C. miles et ha .XII ${ }^{\mathrm{m}}$. pont de pieres

\section{$Z^{\mathrm{t}} 857$}

Primo quidem continebatur in eis quod civitas Qinsay in circuitu suo girat miliaria centum tere, et habet duodecim milia pontium, pro maiori parte de lapidibus, quia aliqui de legnamine sunt constructi

\section{(a2) Norme di Khubilai in merito ad arti e mestieri}

\section{F 1519}

Et si voç di qu'il estoit establi por lor roi qe cascun doie faire les ars de son pere; et, se il avesse. $\mathrm{C}^{\mathrm{m}}$. besanç, ne poroit fair autre ars qe sun pere avoit fait.

\section{$Z^{\mathrm{t}} 85$ 16-18}

Sanccitum erat itaque per Factur, regem dicte civitatis et provincie, tempore quo regnabat, quod quilibet deberet exercere artem sui patris; et si quis haberet centum milia bysantiorum, oportebat ipsum artem patris exercere. Non equidem credatis quod ipsos oporteret propris manibus laborare, sed homines retinere, ut dictum est superius, ad dictam artem exercendam. Sed ad hoc per Magnum Dominum minime compelluntur: nam, si homo artifex in tantas creverit divicias quod artem suam derelinquere possit et vellit, per nullum ad artis exercitium amplius coartatur...

\section{(b1) La pratica del suicidio-vendetta ${ }^{21}$}

\section{$Z^{\mathrm{t}} 85$ 43-46}

Sunt itaque homines provincie Mançi pre alijs gentibus iracundi, et pre ira et dolore ipsimet quam plures aliqui se occidunt. Nam si accidit quod ex ipsis aliquis alteri alicui $i<m>$ primat alapam vel ipsum decapillet aut aliquam sibi inferat iniuriam et gravamen, et iniuriator sit tan potens et magnus quod ille sit impotens ad vindictam, pre nimio dolore passus iniuriam se de nocte ad portam iniuriatoris suspendet et morietur, hoc sibi faciens ad maius vituperium et contemptum...

20 In corsivo, qui e infra, gli addenda.

21 Su questa pratica estrema di rivalsa sociale si rimanda a Barbieri (1998, 214-5 nota 4). 
(b2) L'assedio mongolo e il drago del fiume Qiantang

\section{$Z^{\mathrm{t}} 8562-66$}

Dicimus de quodam miro quod accidit cum Bayan erat in obsidione civitatis istius, videlicet quod cum rex Facfur a facie eius fugit, multitudo gentium ipsius civitatis in navigio fugiebat per quoddam maximum flumen, latum et profundum, quod transit ab uno latere civitatis. Et dum sic per flumen istud fugerent, ipso instanti fuit aqua totaliter desiccatum, ita quod Bayan, hoc inteligens, adivit partem illam, et omnes fugientes ad civitatem redire coegit. Et inventus fuit piscis unus iacens in sicco per transversum alveum fluminis, qui erat quid mirabile ad videndum : nam longitudinis erat bene pasuum centum, grossitudo vero minime longitudini respondebat. Pilosus quidem erat totus, et multi del ipso comederunt, et multi ex ipsis perierunt. Et dominus Marcus, ut dicit, capud illius piscis propriis oculis vidit in templo quoddam ydolorum, etcetera.

\subsection{Il testo in $\mathrm{R}$}

Nei capitoli su Quinsai (R II 68-69), come nel resto della compilazione ramusiana, la fonte più spesso riconoscibile è $\mathrm{Z}^{\mathrm{g}}{ }^{22}$ lo dimostrano le numerose e stringenti coincidenze testuali tra $\mathrm{R} \mathrm{e} \mathrm{Z}^{\mathrm{t}}$ sia in alcuni addenda (es. a1, a2) che nei brani comuni a tutta la tradizione (b1, b2), oltre alla stretta aderenza di $\mathrm{R}$ a $\mathrm{F}$ nei luoghi in cui il Toledano è lacunoso (c): ${ }^{23}$

\section{(a1)}

\section{$Z^{\mathrm{t}} 85$ 16-17}

Sanccitum erat itaque per Factur regem dicte civitatis et provincie, tempore quo regnabat, quod quilibet deberet exercere artem sui patris, et si quis haberet centum milia bysantiorum, oportebat ipsum artem patris exercere. Non equidem credatis quod ipsos propris manibus laborare, sed homines retinere, ut dictum est superius, ad dictam exercendum.

\section{R II 6835}

Et anchor che per li re antichi fosse ordinato per legge che ciascun abitante fosse obligato ad esercitare l'arte del padre, nondimeno, come diventino ricchi, gli è permesso di non lavorar piú con le proprie mani, ma ben erano obligati di tenere la bottega, e uomini che v'esercitassino l'arte paterna.

22 L'indagine microscopica condotta da Alvise Andreose su R II 68 (reperibile ad locum in Simion, Burgio 2015) permette di ipotizzare che $Z^{g}$ sia stato il testo-base per i §§ 30,42, 47-9, 55, 58, 65-70, 91-2; P per i §§ 4, 9, 56-7, 89-90; VB per i §§ 1-3, 36, 63-4, 74. Più fonti concorrono paritariamente alla costituzione del testo nei §§ $32,33,35,53-4,75(Z+P), 34$, $43(\mathrm{P}+\mathrm{VB}), 87(\mathrm{Z}+\mathrm{P}+$ minima da VB), mentre un modello non identificabile (sull'ipotesi che si tratti sempre di $\mathrm{Z}^{\mathrm{g}} \mathrm{cf}$. infra) dà forma alla porzione più consistente del capitolo (§§ 5-8, 10-29, 31, 37-41, 44-6, 50-2, 59-62, 71-3, 76-86, 88). R II 69, invece, è interamente costruito su $\mathrm{Z}^{\mathrm{g}}$, con pochi e minimi/poco significativi apporti da P e VB.

23 In corsivo gli addenda. 
(a2)

\section{$Z^{t} 8535$}

...in aliquibus sunt mille homines, in aliquibus .$X^{m}$., in aliquibus . $X X^{m}$., in aliquibus . $X X X^{m}$., plures et pauciores secundum condicionem terrarum et potenciam earum. (?)

(b1)

\section{$Z^{\mathrm{t}} 8532$}

Item per totam civitatem istam solubrice, idest saleçate, sunt omnes vie et strate de lapidibus et lateribus, et similiter saleçate sunt omnes vie et strate provincie Mançi.

(b2)

\section{Zt 86 1-2}

Ad presens dicere volumus et narare immensam quantitatem reddituum quos Magnus Dominus ab ista civitate percipit et $a b$ aliis terris que civitati isti respondent, que sunt pars nona, sive regnum nonum, provincie Ma`n >çi. Et dicemus primo de sale, quia plus quantum ad reditum valet. (?)

\section{(c)}

\section{R II 6843}

... et quando alcuno vuol celebrar nozze, o vero far qualche solenne convito, va ad uno di questi palazzi, dove gli vien dato tutto quello che per questo effetto gli è necessario, cioè vasellami, tovaglie, mantili et cadauna altra cosa. (?)

\section{R II 6869}

...in alcune [città] vi saranno mille huomini, in altre diecimila o vero ventimila, secondo che 'I giudicherà che quella città sia piú et manco potente. (?)

\section{R II 6849}

Et prima è da sapere che tutte le strade di Quinsai sono saleggiate di pietre e di mattoni, e similmente sono saleggiate tutte le vie e strade che corrono per ogni canto della provincia di Mangi. (?)

\section{R II 691}

Hor parliamo alquanto della entrada che ha il Gran Can della città di Quinsai et dell'altre a quella adherenti: il Gran Can riceve da detta città et dall'altre che a quella rispondono, che è la nona parte o vero il nono regno di Mangi; et prima del sale, che val piú quanto alla rendita. (?)

\section{F 15112}

Et quant l'en vuelt faire noses ou convit, il vunt a ceste palais et la font lor noses et lor feste, et iluec treuvent toutes les aparoillement qe beçongne au convive, ce est de vaicellemant et de tailleor et d'escueles. (?)

Se R II 69 riproduce la lezione di $\mathrm{Z}^{\mathrm{t}}$ in maniera pedissequa, più complessa è l'interpretazione di R II 68, che rimane l'episodio più enigmatico dell'intera compilazione ramusiana: rispetto all'intera tradizione, infatti, il capitolo si 
caratterizza per la presenza di numerosi ed estesi unica, perlopiù di taglio descrittivo (approfondimenti sulla topografia della città, cenni di architettura ed edilizia, spaccati di vita cittadina), la cui inserzione in vari punti del testo produce modifiche radicali nell'ordine degli argomenti (cf. tav. 2).

Le aggiunte singolari di $\mathrm{R}$ offrono informazioni dettagliatissime e, laddove le si possa verificare, del tutto coerenti con la realtà storica, geografica e sociale della Quinsai del secondo Duecento. Sono per esempio attendibili, a dispetto di quanto è stato spesso rilevato, i passi esclusivi che Ramusio dedica ai bagni pubblici di Quinsai: il testo di R pone l'attenzione sulla consuetudine degli abitanti della città di lavarsi con acqua fredda, mentre in F 15119 e nel resto della tradizione - $\mathrm{Z}^{\mathrm{t}}$ purtroppo qui è compendioso - si fa riferimento ai soli bagni caldi. La nota sui bagni freddi, tacciata di inesattezza da Yule («This is in contradiction with the notorious Chinese horror of cold water for any purpose», Yule, Cordier 1903, 2: 246) e per questo addotta a prova della scarsa attendibilità di alcune aggiunte di R, è invece accolta come genuina da Gernet e trova qualche pezza d'appoggio - lo segnala Schafer - nella documentazione del periodo Ming (Schafer 1956, 70-1; Gernet 1959, 135-6). Corrette sono anche le informazioni sui ponti carrabili (a) e sulle carrozze da diporto (b), peraltro confermate - con qualche sorprendente coincidenza testuale - da un paio di passi del Mengliang lu: $:^{24}$

\section{R II 68}

Hor sopra questa strada di continuo si veggono andar su et giú alcune carrette lunghe, coperte et acconcie con panni et cussini di seda, sopra le quali vi possono stare sei persone, et vengono tolte ogni giorno da huomini et donne che vogliono andare a solazzo: et si veggono tutt'hora infinite di queste carrette andar a lungo di detta strada per il mezzo di quella, et se ne vanno a' giardini

\section{R II 68}

[I ponti] che sono fatti sopra i canali maestri et la strada principale sono stà voltati tanto alti [...] et nondimeno vi passano sopra carrette et cavalli, talmente sono accommodate piane le strade con l'altezza.

\section{Mengliang lu, cap. 2 (trad. Moule)}

The carriages and horses going and coming in vast numbers block the gates of the capital. Those who picnic in the suburbs go to famous gardens, beautiful parks, or places where there are strange flowers and rare trees [...] At this time (very early in the morning) the processioni is still going on.

\section{Mengliang lu, cap. 7 (trad. Moule)}

The Chung-an and Kuan bridges are both level with the Imperial Street, and so at the four quarter-days the Emperor's coaches pass by way of these two bridges. 
Di queste informazioni peculiari di R, almeno due - la grande quantità di viveri spacciata nei mercati di Quinsai (§§ 29-30) e le gite lacustri (458 ) - sono certamente riconducibili a $\beta^{\prime \prime \prime}$, stante la sopravvivenza di qualche loro traccia (rispettivamente ai §§ 60-1 e 26-7) nel compendioso Toledano. Ma anche ammesso di voler attribuire tutti i singularia di R alla 'fase Z', giustificando la loro assenza in $\mathrm{Z}^{\mathrm{t}}$ con le riduzioni operate dallo scriba, restano da sciogliere due nodi complicati: (1) la disposizione eccentrica delle tessere di $\mathrm{R}$, diversa da quella di $\mathrm{Z}^{\mathrm{t}}$ ( = F et alii) e al contempo difficilmente attribuibile all'iniziativa dell'editore; ${ }^{25}$ (2) la mancata corrispondenza tra $\mathrm{R}$ e $\mathrm{Z}^{\mathrm{t}}$ in tre passi (qui di seguito alle lettere $\mathrm{a}, \mathrm{b}, \mathrm{c}$ ) nei quali il Toledano, non abbreviato, procede di pari passo con $\mathrm{F}$ e la tradizione, mentre $\mathrm{R}$ testimonia vere e proprie versioni alternative.

(a) La descrizione dei ponti ${ }^{26}$

\section{F 1517 \\ $Z^{t} 85$}

... la cité de Quinsai gir'environ.C.

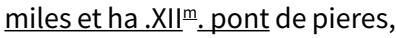
et por chascun de cesti pont, ou por la greingnor partie, poroit bien passer une (grant) nes por desout sun arche, et por les autres porroient passer mesn idre nes. Et nulz se face mervoille se il ha tant de pont, por ce qe je vos di qe ceste ville est toute en eve et est environcés de eve, et por ce convient qe maint pont hi aie por aler por toute la ville
... civitas Qinsay in circuitu suo girat miliaria çentum tere, et habet duodecim milia pontium, pro maiori parte de lapidibus, quia aliqui de legnamine sunt constructi; sub singulis quorum, vel pro maiori parte, transiret navis una, sub arcu, grandis et magna, per alios vero possent transire minores. Et nullus miretur si tot sunt ibi pontes: nam civitas tota est sita in aquis, et circundata aquis; et ideo necesse est ut sint ibi tot pontes ad hoc ut gentes possint per civitatem quacumque pertransire

\begin{abstract}
R II 68
Questa città, per commune opinione, ha di circuito cento miglia, perché le strade et canali di quella sono molto larghi et ampli; poi vi sono piazze dove fanno mercato [...] Et è fama che vi siano dodicimila ponti, fra grandi et piccioli: ma quelli che sono fatti sopra i canali maestri et la strada principale sono stà voltati tanto alti e con tanto magisterio [<VB?] che una nave vi puol passare di sotto senza albero $[<\mathrm{P}]$; et nondimeno vi passano sopra carrette et cavalli, talmente sono accommodate piane le strade con l'altezza. Et se non vi fussero in tanto numero non si potria andar da un luogo all'altro $[<\mathrm{P}]$

Dall'altro canto della città v'è una fossa...
\end{abstract}

25 Ramusio si attiene solitamente all'ordo di uno dei suoi modelli; gli spostamenti di pericopi sicuramente attribuibili alla sua iniziativa sono piuttosto rari e di solito riguardano porzioni di testo contenute.

26 Qui e negli esempi successivi, in carattere sottolineato i segmenti comuni alle tre redazioni, in grassetto quelli che accomunano R-Z $\mathrm{Z}^{\mathrm{t}}$ escludendo P-VB, in corsivo gli unica di R. 


\section{(b) Il consumo della carne}

\section{F 15114}

Et si vos di qe les jens sunt ydres et sunt au Grant Kan, et ont monoie de carte.

Il menjuent tutes cars, et de chiens e de toutes autres brutes bestes et autres animaus que por ren dou munde nul cristienç de sa ne menjuerént

\section{$Z^{t} 85$ 28-9}

Gentes adorant ydolla, monetam habent de cartis et sunt sub dominio Magni Can.

Comedunt omnes carnes, canum et generaliter omnium animalium

\author{
R II 68 14-5 \\ Vi sono poi le beccarie, dove \\ ammazzano gli animali grossi, \\ come vitelli, buoi, capretti et \\ agnelli, le qual carni mangiano \\ gli huomini ricchi et gran maestri; \\ ma gli altri che sono di bassa \\ conditione non si astengono da \\ tutte l'altre sorti di carni immonde, \\ senza havervi alcun rispetto
}

\section{(c) Il palazzo dell'imperatore Song}

\section{F 151 28-31}

Et en ceste cité est le palais dou roi qe se fuï, qe seingnor estoit ou Mangi, qui est le plus biaus e le plus noble qe soit au monde; e vos en diviserai aucune cousse. Or sachiés qe le palais gire environ .X. miles et est murés cun autes mures, toutes as querriaus, et dedens as mures sunt maint biaus jardis con tuit les buens fruit qe home seust deviser. Il hi a maintes fontaines et plusors lac, la o il ‘a〉 maint buen peison. Et, eu mileu, est le palais mout grandissme et biaus. Il a une si gran sale et si belle, qe grandisme quantité des jens hi poroient demorer et menjuere a table. La sale est toute portraite et pointe a penture d'or, et hi a maintes estoires et maintes bestes et hosiaus et chevalers et dames, et a maintes mervoilles. Il est mout bielle viste a garder, car en toutes les murs et en toutes covreoure ne poroit l'en veoir che pintures a or. Et que voç en diroie? Sachiés qe je ne vos poroie deviser la gran nobelité de cesti palais, mes je voç en dirai brefmant et sommeemant tout la verité. ¿Sachiés` de voir qe cest palais a .XX. sales, toutes d'une grant et d'un paroil; et sunt bien si grant qe. $X^{m}$. homes hi poroient menuier a table aaisemant; et sunt toute pointe a ouvre d'or mout noblemant. Et si voç di qe ceste palais ha bien .M. canbres, ce sunt maison bielles et grant, e de dormir et de mengier. Les frut et les pesciere vos ai contés.

\section{$Z^{t} 85$ 49-57}

In ista quidem civitate Qinsay est regale palatium, quod fuerat regis Facfur, domini provincie Manci, quod est pulcrius et nobilius aliquo quod reperiatur in mundo; de cuius facturis dicemus. Circuit enim palatium istud bene miliaria sex de terra. Est itaque altis muris valatum, et intra muros sunt multa pulcra viridaria omnimodos producentia bonos fructus. Sunt et ibi pulcri fontes et lacus quam plures, in quibus inveniuntur in habundantia boni pisces. Et in medio istius muri est palatium, valde pulcrum et magnum. In eo est quedam magna salla magistra, in qua simul discumberent multe gentes. Sala ista depicta est tota aureis picturis, $\underline{\text { ubi }}$ sunt ystorie diverse, bestie, aves, milites et domine cum multis mirabilibus; quod est pulcer intuitus ad videndum, quoniam in toto muro et omnibus coperturis, non posunt videri nisi solummodo ystorie colorate auro et aliis coloribus delectabilibus et pulcris. Palatium istud multas habet salas omnes spatiosas et pulcras, depictas ad aurum et subtiliter laboratas.

Habet insuper cameras bene mille; et est mirabile quid esse istius palatii. 


\begin{abstract}
R II 68 75-85
Hor parleremo d'un bellissimo palazzo dove habitava il re Fanfur, li predecessori del qual fecero serrare un spatio di paese che circondava da dieci miglia con muri altissimi, et lo divisero in tre parti. In quella di mezzo s'entrava per una grandissima porta, dove trovavansi da un canto e dall'altro loggie a piè piano grandissime et larghissime, col coperchio sostentato da colonne, le quali erano dipinte et lavorate con oro et azzurri finissimi; in testa poi si vedeva la principale et maggior di tutte l'altre, similmente dipinta con le colonne dorate, et il solaro con bellissimi ornamenti d'oro, et d'intorno alle parieti erano dipinte l'historie di re passati, con grande artificio. Quivi ogni anno, in alcuni giorni dedicati alli suoi idoli, il re Fanfur soleva tenir corte et dar da mangiare alli principali signori, gran maestri et ricchi artefici della città di Quinsai: et ad un tratto vi sentavano a tavola commodamente sotto tutte dette loggie diecimila persone. Et questa corte durava dieci o dodici giorni, et era cosa stupenda et fuor d'ogni credenza [...] cadauno claustro o vero corte havea cinquanta camere con li suoi giardini, et in tutte queste camere vi stantiavano mille donzelle che 'I re teniva alli suoi servitii; qual andava alcune fiate, con la regina et con alcune delle dette, a sollazzo per il lago, sopra barche tutte coperte di seda, et ancho a visitar li tempii degl'idoli. Le altre due parti del detto serraglio erano partite in boschi, laghi et giardini bellissimi, piantati di arbori fruttiferi, dove erano serrati ogni sorte di animali, ciò̀ caprioli, daini, cervi, lepori, conigli: et quivi il re andava a piacere con le sue damigelle, parte in carretta et parte a cavallo [...] Tutta questa narratione mi fu detta da un ricchissimo mercatante di Quinsai, trovandomi in quella città, qual era molto vecchio et stato intrinseco familiar del re Fanfur, et sapeva tutta la vita sua et havea veduto detto palazzo in essere, nel qual volse lui condurmi
\end{abstract}

\title{
3 Conclusioni
}

Si può discutere, a questo punto, di alcune ipotesi.

In primo luogo, pare difficile credere che Ramusio, nei capitoli su Quinsai, abbia interpolato la lezione dei suoi relatori poliani con una fonte odeporica 'non poliana' e non altrimenti nota, dalla quale avrebbe desunto la gran mole di informazioni esclusive: cosa che, tra l'altro, sarebbe in contrasto con le proposizioni di metodo dichiarate nella dedicatoria a Fracastoro posta in limine all'edizione. ${ }^{27}$

Altrettanto improbabile è che abbia attinto, per questa sola sezione, a un'ulteriore, non identificata redazione del Devisement, della quale, peraltro, non si rinviene traccia nel resto del libro.

Rimane, se non ci si inganna, una sola possibilità, che ha se non altro il pregio di evitare la moltiplicazione degli enti: e cioè che uno dei manoscritti ( $\mathrm{Z}^{\mathrm{g}} \mathrm{P}$ VB L V) serviti a Ramusio per costruire la sua edizione testimoniasse, all'altezza del trattato su Quinsai, una lectio singularis: ovvero, un testo profondamente rielaborato nella struttura e ricchissimo di informazioni inedite.

$27 \ll \ldots$ cosa ragionevole ho giudicato di far venir in luce il suo libro, col mezo di diversi esemplari scritti già piú di dugento anni, a mio giudicio perfettamente corretto e di gran lunga molto più fidele di quello che fin ora si è letto» (Dedicatoria al Fracastoro, § 10, in Simion, Burgio 2015). 
Che l'esemplare in questione vada individuato nel codice Ghisi è, naturalmente, l'ipotesi meno onerosa, stante la spesso disorientante mouvance testuale riscontrabile nei testimoni direttamente o indirettamente inquadrabili entro la 'costellazione Z' (cf. § 1). Si può dunque supporre - tornando alla proposta formulata in coda al $\S 1.2$ - che, a valle di $\beta^{\prime \prime \prime}$, uno degli antecedenti di $\mathrm{Z}^{\mathrm{g}}$ (o $\mathrm{Z}^{\mathrm{g}}$ stesso?) sia stato soggetto a una parziale riscrittura che avrebbe comportato l'innesto, su uno scheletro simile a quello di $\mathrm{Z}^{\mathrm{t}}$, di materiali testuali nuovi e soprattutto di prima mano. E del resto probabile che Marco Polo, o qualcuno dei suoi familiari, avesse conservato una grande quantità di informazioni su Quinsai sotto forma di appunti e ricordi, sfruttati solo parzialmente nella prima stesura del livre e successivamente rifusi in uno (o più?) esemplari $Z$, con o senza la sua supervisione. In alternativa - ma pare una soluzione meno economica - si dovrà credere che a Venezia, tra Tre e Quattrocento, circolassero altre raccolte odeporiche (zibaldoni di viaggiatori?) contenenti descrizioni di Quinsai, ${ }^{28}$ alcuni brani delle quali potrebbero essere stati interpolati nel testo di $\mathrm{Z}$ a integrazione del dettato poliano.

28 A questo proposito, nella seconda redazione della Relatio accolta nelle Navigationi di Ramusio, Odorico segnala di avere «ben trovate in Vinetia assai persone che vi [a Quinsai] sono state» (Milanesi 1978-88, 4: 311) 


\section{Tavole}

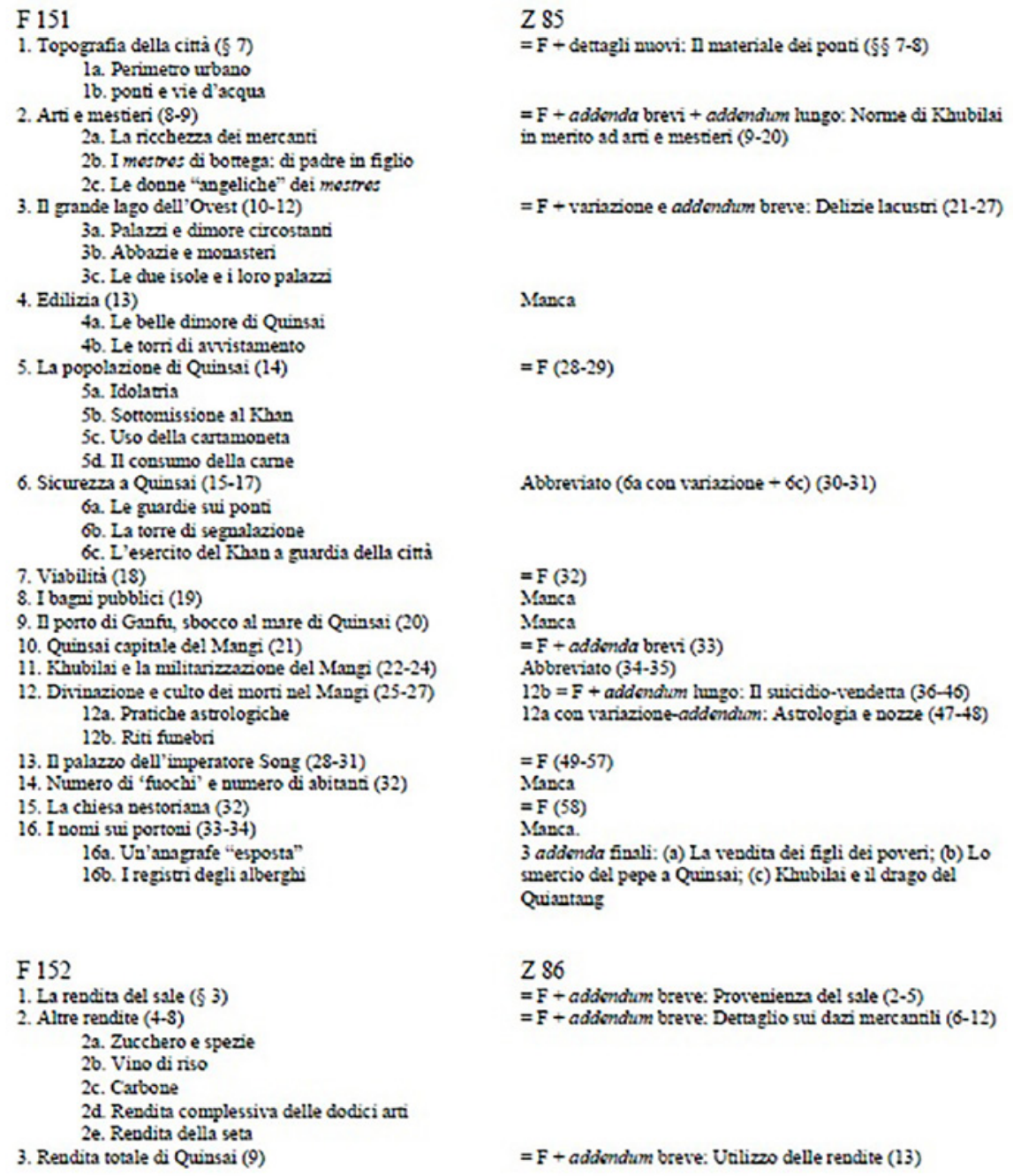

\section{F 151}

1. Topografia della città (§ 7$)$

1a. Perimetro urbano

1b. ponti e vie d'acqua

2. Arti e mestieri (8-9)

2a. La ricchezza dei mercanti

2b. I mesres di bortega: di padre in figlio

2c. Le donne "angeliche" dei mestres

3. I grande lago dell' Orest (10-12)

3a. Palazzi e dimore circostant

3b. Abbazie e monasteri

3c. Le due isole e $i$ loro palazzi

4. Edilizia (13)

43. Le belle dimore di Quinssi

4b. Le torri di atvistamento

5. La popolazione di Quinsai (14)

53. Idolatis

5b. Sottomissione al Khan

5c. Uso della cartamoneta

5d. Il consumo della came

6. Sicurezza a Quinsai (15-17)

6a. Le guardie sui ponti

66. La torre di segnalazione

$6 c$. L'esercito del Khan a guardia della citta

7. Viabilita (18)

8. I bagni pubblici (19)

9. I porto di Ganfu, sbocco al mare di Quinsai (20)

10. Quinssi capitale del Mangi (21)

11. Khubilai e la militarizzavione del Mangi (22-24)

12. Dixinazione e culto dei morti nel Mangi (25-27) 12a. Pratiche astrologiche

12b. Riti funebri

13. In palazzo dell'imperatore Song (28-31)

14. Numero di 'fuochi' e numero di abitant (32)

15. La chiesa nestoriana (32)

16. I nomi sui portoni (33-34)

16a. Un'anagrafe "esposta"

106. I registri degli alberghi

\section{F 152}

1. La rendita del sale (§ 3)

2. Altre rendite (4-8)
2a. Zucchero e spezie
2b. Vino di riso
2c. Carbone
2d. Rendita complessiva delle dodici arti
2e. Reodita della seta

3. Reudita totale di Quinsai (9)

\section{Z 85}

= F + dettagli nuovi: I materiale dei pouti (\$ई 7-\$)

= F + addenda brevi + addendion lungo: Norme di Khubilai in merito ad arti e mestieri $(9-20)$

= F + variazione e addendum breve: Delizie lacustri (21-27)

Manca

$=\mathrm{F}(28-29)$

Abbreviato (6a con variazione $+6 c)(30-31)$

$=\mathrm{F}$ (32)

Manca

Manca

$=\mathrm{F}+$ addenda brevi (33)

Abbretiato (34-35)

$12 \mathrm{~b}=\mathrm{F}+$ addendum lungo: I suicidio-vendetta (36-46)

12 a con variazione-addendion: Astrologia e nozze $(47-48)$

$=\mathrm{F}(49-57)$

Manca

$=\mathrm{F}(58)$

Manca.

3 addenda finali: (a) La vendita dei figli dei poveri; (b) Lo smercio del pepe a Quinsai; (c) Khublai e Il drago del Quiantang

Z 86

=F + addondum breve: Provenienza del sale (2-5)

= F + adicndum breve: Dettaglio sui dazi mercancli (6-12)

=F + addondum breve: Utilizzo delle reodite (13)

Tavola 1. Sinossi F 151-2 / Z 85-6 


\section{RII $6 \mathrm{~S}$}

1. Topografia della citra + addonda:

(a) Anpiezza di canali e strade: (o) Lazo, fumse, canali navigabiit: (c) Strutara dei pont: (d) Canslizazioni e govemo delle acoue: (e) Merci, meecanti, mercati (ingloba 5d. Il consumo della carne, nelaborato. e S. I

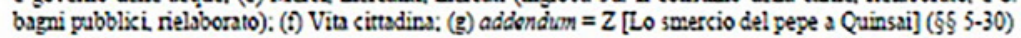

5a. Idolatria (31)

5c. Uso della caramoneta (31)

addendum lungo: Le vesti di seta (31)

2. Arti e mestieri, con addenda brevi $=Z(32-35)$

42. Le belle dimore di Quinsai (36)

addendion lungo: Mansuerudine degli abitanti di Quinsai (37.41)

3. I lago di Mezzogiorno + addendum lungo, con elementi $=Z$ [Delizie lacustri] (42-48)

7. Viateliti - addendum lungo: Il traffico stradale a Quinswi (49-52)

12. Dixinszione e culto dei morti nel Mangi con elementi $=Z$ [Astrologis e nozze] (53-56)

4b. Le torri di avvistamento (57)

6a. Le guardie sui ponti - addenda: (a) La segnalazione delle ore e il coprifuoco: (0) Ricovero delle merci in caso di inserdio (<VB) (58-63)

6c. L'esercito del Khan a guardia della citti (64)

6b. La torre di segaslazione (65)

10. Quinsai capitale del Mangi. con addenda brevi $=Z(66-68)$

11. Khublas e la militarizariose del Mangi + addendum lungo: I Tarari e il reciutamento dell'esercito nei docmini cinesi (69-74)

13. Il pulazzo dell' imperatore Sung, nelaborato con adienda (75.86)

9. Il porto di Ganfu, sbocco al mere di Quinssi + adienda brevi (87.88)

14. Numero di "fuochi" e numero di abitanti (\$9)

15. La chiesa nestoriana ( 89 )

16. I portoni della citta come registri d' anugrafe (90-91)

Addondum = Z: La vendita dei figli dei poveri (92)

Tavola 2. R II 68: struttura e contenuti 


\title{
Filologia dei testi volgari e ambienti digitali Qualche annotazione a margine del Ramusio digitale
}

\author{
Eugenio Burgio \\ (Università Ca' Foscari, Venezia, Italia)
}

\begin{abstract}
The paper presents some reflections on the use of digital applications in the field of textual philology, which particularly concerns the edition of medieval texts in vernacular.
\end{abstract}

Sommario 1 Premessa. - 2 Ricostruire o conservare? Dialettiche ecdotiche in ambiente digitale. -3 SDE, o della profilassi contro il «iudicium». - 4 Sul Ramusio digitale (per chiudere).

Keywords Digital Philology. Edition of medieval texts. Vernacular. Devisement dou monde. Ramusio.

\section{Premessa}

Non sono sicuro di essere il ricercatore più adatto a offrire qualche annotazione interessante sul tema indicato dal titolo. L'incipit può apparire insopportabilmente civettuolo, ma il fatto è che - nonostante Ramusio e la sua edizione ipertestuale - non sono un fedele settatore della Digital Scholarly Edition (DSE, per comodità di tutti), ma solo un frequentatore casuale di quegli ambienti, a cui mi sono avvicinato per trovare una soluzione a uno specifico problema filologico/editoriale: come ha spiegato bene Samuela Simion nel contributo in questo volume, volevamo tenere insieme su un unico supporto la visibilità (nel senso di 'leggibilità', ma non solo) di una serie di oggetti storicamente identificabili - alcune edizioni medievali del Devisement dou monde/Milione - e il doppio processo testuale che generarono e alimentarono un nuovo oggetto (l'edizione dei Viaggi, un'editio variorum di stile umanistico) e un nuovo commento (nel quale l'analisi filologica delle procedure di inventio e dispositio sintagmatica dei contenuti, attivate da Ramusio nella stesura del 'suo' Milione, sono state modulate nella mise en forme della glossatura continua, aderente alle più minute articolazioni del testo commentato).

Insomma, ci interessava (a me, e al gruppo dei 'commentatori' che tra il 2009 e il 2015 ha lavorato al progetto: cf. Burgio 2011) più la dimensione empirica che il 'discorso sul metodo' inaugurato dalla New Philology (e il suo portato di oltranzismo teorico: i termini della questione in Leonardi 2007, 66 e note 3-5). Questa attitudine ci ha condotto ad adottare il 
modello dell'ipertesto, che per le sue caratteristiche - l'interattività, la multilinearità, l'apertura alla polidimensionalità, la capacità di 'mimare' l'idea stessa di 'processo' (cf. Fiormonte 2003, 81 ss.; Roncaglia 2010, 216 ss.) - ci pareva lo strumento più duttile ai nostri scopi; e lo abbiamo usato, in un certo senso, come alternativa temperata al codice glossato. Retrospettivamente, direi che il succo del progetto consiste in due punti, che esporrò qui in maniera un po' sbrigativa. (1) Il Ramusio ipertestuale condivide con il codice scolastico del XII-XIII secolo (e con il suo layout) un'attitudine tabulaire nei confronti del testo: la linearità di quest'ultimo (qui posta al centro della pagina, lì mantenuta alla voce «Testimoni») è trasformata (per frantumazione/ricomposizione) in una superficie nella quale i contenuti e le loro articolazioni sono colti attraverso una griglia di riferimenti incrociati (Vanderdorpe 1999, 54-6); (2) d'altra parte, l'ipertesto rende visibile la struttura tabulare del testo (e del commento), e la trasforma in un dinamismo che è una parte ineludibile (per il filologo come per i lettori) del significato dell'opus filologico (e intendo: quello di Ramusio e quella dell'équipe della sua versione digitale): perché il mezzo è il significato, come i teorici del Great Divide hanno persuasivamente spiegato (cf. De Kerckhove 2008). Su questi due punti poggia anche la questione nella quale vorrei riassumere le mie annotazioni: in che misura il Ramusio ipertestuale partecipa della proclamata «Digital Revolution in Scholarly Editing»? (cf. Robinson 2016, e qui le osservazioni di Simion).

Ovviamente, la questione non è proprio di lana caprina, e può essere affrontata da diversi punti di vista: a cominciare dal fatto che - con Robinson $(2016,181)$ - possiamo e dobbiamo «argue that the changes we may see in scholarly editing may amount to a revolution». Tralasciando per il momento sia il polimorfismo della definizione corrente di digital critical edition (e più in generale di digital philology: vedi la bibliografia raccolta qui da Simion alle note 3-4), sia la riluttanza della tribu filologica - almeno in certe province continentali degli studi umanistici - ad aderire alle parole d'ordine della Rivoluzione digitale, bisognerà ammettere che non è del tutto evident «that something is radically changing in the scholarly editing world [...] with respect to the older print-based workflow»: «the way we work, the tools we use to do such work and the research questions to which we try to give answers» (Driscoll, Pierazzo 2016b, 3). Per cominciare, proviamo a guardare la cosa da chi/dal punto di vista di chi - tutti noi, in determinati momenti del nostro lavoro di ricerca (ma pure in quelli più gratuiti del puro e semplice «Wasting Time on the Internet»: Goldsmith 2017) - ricorre alla rete come archivio di informazioni: non c'è dubbio che la massa di «electronic versions of cultural heritage texts» (e di oggetti iconici, e di banche-dati) disponibili liberamente su Internet (Driscoll, Pierazzo 2016b, 6) superi anche la più ingorda delle aspettative, e semplifichi/arricchisca il nostro lavoro intellettuale (Dio, o chi per lui, benedica Google, per tutte le volte che, durante una lezione, in un'aula dotata di connessione wi-fi 
possiamo inseguire e dare corpo all'uzzolo del momento, allo scoccare improvviso del ricordo di un'immagine, di un testo...). Ma, al postutto, tendiamo a muoverci nell'ecosistema digitale come se fosse una biblioteca di tipo analogico: cerchiamo (e troviamo) testi e immagini impaginati come se fossero su supporti cartacei; e ammesso che «the experience of reading a text on the screen of a mobile phone can be radically different from that of a tablet or a laptop», e a che a fronte dello stesso testo, «yet the reading experience (and ultimately the message) can vary greatly» (Pierazzo 2016, 51 ), le azioni in cui con maggiore frequenza condensiamo questa nuova forma di lettura sono scaricare testi e immagini, e stamparli su supporti cartacei... Sono habitus nuovi, attribuibili all'interazione con un medium diverso da 'the older print-based workflow'? Difficile dirlo. La sensazione è che, almeno per questi habitus, ci muoviamo in quella zona grigia in cui il mutamento quantitativo non è ancora divenuto mutamento qualitativo...

Le cose prendono un'altra piega se ci interroghiamo sulla natura (le virtutes) dell'enorme massa dei testi e degli oggetti culturali che affollano Internet, e se poniamo la questione osservando questa massa attraverso le lenti del filologo, consapevole che il suo «compito primario» è «produrre un testo affinché sia letto» (Leonardi 2007, 73). Paola Italia (2016a) ha scritto nel merito osservazioni molto sensate, che richiamerò qui, concentrandomi su due delle sue argomentazioni, che distinguono i testi a partire dal supporto per cui sono pensati. (1) I testi prodotti esclusivamente per la Rete hanno, è noto, natura proteiforme: il medium attribuisce al loro autore il potere di sottoporli a illimitata riscrittura, e destinarli così a una potenzialmente perpetua mobilità. Non è un'avventura indolore, perché sul campo restano, come avverte Italia (2016a, 81-2), alcuni morti: non solo l'editing (attività che molto a che spartire con la prassi filologica) e chi lo pratica («perché l'autore è diventato editor di se stesso»), ma anche una certa idea di testo:

La perfezione del testo, quella verità che ogni redattore del secolo scorso ha inseguito come chimera della sua deontologia professionale [...], diventa la perfettibilità di una continua correzione, ma anche l'impossibilità di sapere, del testo pubblicato in rete in un blog, in un giornale on line, in un sito web, quale sia la sua ultima forma storica, la sua definitiva veste linguistica e, in ultima analisi, la sua verità testuale. (Italia 2016a, 82)

Sono parole che, al filologo di testi volgari medievali, ricordano tutto il dibattito novecentesco che da Bédier si è sviluppato fino alle ultime propaggini della 'New Philology' sulla mouvance che agita i testi trasmessi in tradizioni pluritestimoniali, e sulla sua sedicente alterità rispetto all'idea di Testo trasmessa dalla pratica intellettuale umanistica alla Modernità (rinvio ancora a Leonardi 2007)... Più in generale, esse richiamano una 
delle pièces de résistance invocate dai teorici della 'liquidità' digitale del Testo; ma il dato interessante in questo caso è che Italia fa riferimento a un aspetto di solito trascurato in quella tematizzazione, e cioè il diritto del lettore di sapere in che testo si trova; in altri termini, il diritto di essere informato sulle modificazioni subite dal testo: che è poi, allargando per un istante il fuoco dell'argomentazione, quanto normalmente ci si aspetta - se ne si è consapevoli - dal lavoro del filologo. (2) In un certo senso, insomma, di fronte agli effetti del Great Divide Italia attribuisce alla filologia il compito di essere più amichevole nei confronti del lettore. «Stiamo muovendo sempre più da una filologia per l'autore a una filologia per il lettore» (Italia $2016 a, 84)$ è un'affermazione che sembra più una invocazione ai filologi che una rivendicazione per i filologi: i quali dovrebbero interrogarsi seriamente (agendo di conseguenza) su che fare di fronte alla politica di Google e alla migrazione sulla Rete di testi nati e pensati per il supporto cartaceo (e per secoli a questo destinati). Digitalizzando oltre la metà del patrimonio librario esistente, offrendo all'internauta i multipli editoriali di uno stesso testo ma non un filtro bibliografico-filologico (ah, i metadati!) adeguato a questa molteplicità, Google non solo ha scardinato, senza fanfare teoriche, l'idea stessa di Canone (qui sì il mutamento quantitativo ha generato uno scarto qualitativo), ma ha lasciato il 'lettore comune' solo di fronte alla domanda «che testo legge quando legge un testo?» (Italia 2016a, 83). Giustamente Italia dispone questa incontrollabile ricchezza testuale/ libraria entro la polarità 'manicaretti vs junk food'; di fronte all'alternativa, il pallino torna in mano ai filologi: che forse dovrebbero decidere per chi lavorano, se solo per i 'Parigini' o anche (un pochino) per gli 'Ottentotti'.

\section{Ricostruire o conservare? Dialettiche ecdotiche in ambiente digitale}

L'inventario redatto da Patrick Sahle (2017) conta circa quattrocento edizioni digitali riconducibili a metodologie ecdotiche: come ha già notato Simion (alla nota 2 del suo contributo in questo volume), i loro oggetti sono in maggioranza testi otto-novecenteschi, e in ambito medievale le edizioni di opere a tradizione monotestimoniale dominano su quelle trasmesse da più testimoni. Aggiornato al 2017, l'inventario conferma linee di tendenza evidenziate da Leonardi (2007) e più recentemente da Italia (2016b). Procedo anche qui un po' alla grossa.

(1) In questi ultimi vent'anni la filologia digitale sembra aver prediletto i testi moderni, e l'applicazione meno della prassi della 'filologia d'autore' che dei principi della Critique génétique. Come ha indicato Italia (2016b, 247-8), 
nella filologia d'autore [...] è centrale la volontà (se non il dovere) di presentare al lettore un 'testo', rispetto al quale si deve stabilire la variazione genetica e/o evolutiva dell'apparato, per una funzione comunicativa e didattica (se non continianamente pedagogica); nella critica genetica prevale invece la rappresentazione del flusso correttorio 'eracliteo', dove testo e apparato convivono e vengono rappresentati contemporaneamente, nella riproduzione tipografica e/o digitale che mima il movimento del testo stesso.

E non c'è dubbio che la Critique génétique - con una certa simmetria di attitudini intellettuali con la 'New Philology' - valorizzi la costituzione di «un testo fluido, mobile, processuale, che si avvicin[a] alla fluidità del testo in rete, e che si contrappone al concetto di testo fisso, stabile e definito, invalso nell'era 'analogica'» (Italia 2016b, 247; e cf. Sahle 2016, 29: una fluid publication digitale «is a process rather than a product» [corsivo nell'originale]).

(2) D'altro canto, come dicevo, la più parte delle edizioni medievali registrate nel repertorio di Sahle riguardano testi trasmessi da codices unici: i teorici della DSE le definiscono «documentary digital editions» (Pierazzo 2011), o «image-based scholarly editions» (Kiernan 2006). Come spiega Robinson $(2016,191)$ - a cui si deve il referto lessicografico appena citato (cf. 192 ss.) -,

many of these editions are what one might call digital facsimile transcripts, focussing on a single manuscript and recording its text in precise form, page by page, line by line and character by character. These editions - and they most certainly are editions, in the basic sense that an editor is scrutinizing every mark on the page - characteristically focus on two elements: the exact disposition of the text on each page and on the writing process.

In esse - ma in qualche misura anche nelle edizioni che affrontano tradizioni ad attestazione plurima (ad esempio i progetti relativi alla Queste del saint Graal: cf. Marchello-Nizia, Lavrentiev, Guillot-Barbance 2015, e all'Hêliand: cf. Buzzoni 2011) - è facilmente riconoscibile una sorta di «feticismo del Documento» (Italia 2016b, 248; e cf. Leonardi 2007, 67), che si esibisce in una minuziosissima riproduzione della sua fisionomia, dall'assetto grafematico a quello paratestuale (frequentemente posta in relazione immediata e diretta alla riproduzione ad alta risoluzione del manufatto originale), e nel tentativo di sciogliere nella adesione mimetica alla copia fotografica la distinzione tra testo e trascrizione, e di far coincidere la verità del primo nella forma della seconda. Da qui due modalità intellettuali, ben riconoscibili in buona parte dell'argomentazione di Robinson 
(2016, 184 ss.): l'attenzione ossessiva nei confronti della codifica digitale dei caratteri - grafematici, decorativi, ecc. - della copia (come sa bene chiunque frequenti, anche da lontano, i dibattiti che agitano la setta della Text Encoding Initiative (TEI: cf. Italia 2016b, 249-50); la corrispondente perdita di consapevolezza della distinzione tra fatti formali e fatti sostanziali (una distinzione che è un caposaldo della filologia ricostruttiva almeno dall'edizione di G. Paris della Chanson de saint Alexis (Paris, Pannier 1872).

Com'è noto, il dibattito novecentesco sull'art d'éditer i testi del Medioevo volgare si è costruito intorno a una polarità fondamentale, «l'alternativa tra conservazione e ricostruzione, tra la sincronia del singolo manoscritto e la diacronia genealogica dello stemma, tra la verità del copista e la verità dell'autore» (Leonardi 2007, 65). Gli esiti di vent'anni di digital philology suggeriscono che la più parte dei suoi cultori propenda nettamente per il polo della conservazione (e in conseguenza, per il lavoro su testi a tradizione unitestimoniale). Vari fattori hanno probabilmente contribuito al cristallizzarsi di questa attitudine nella prassi e nella riflessione teorica (ché un tratto costitutivo della disciplina è la sua immediata e fortissima pulsione a darsi una specifica autorappresentazione: «doing things digitally is not simply doing the same old thing in a new medium. In addition, it seems that not only have the methods changed, but this new medium requires a fair bit of theoretical re-thinking and reflection on the significance of what we are doing and its impact on the discipline and on out notion of textuality», Driscoll, Pierazzo 2016b, 9). Innanzitutto, non è stato insignificante il gioco di continuo va-et-vient intellettuale con le tematizzazioni della 'New Philology' (a cominciare dal suo acclamato incunabolo, Cerquiglini 1989), e con la sua emphasis "on the 'real' text as it has been preserved, received, annotated, and used» (Andrews 2013), che spesso nasconde (come vedremo subito) una rappresentazione macchiettistica delle fasi artigianali del lavoro del filologo. E non vanno sottaciute le difficoltà di ordine pratico che i progetti di edizioni digitali (maggiori, ovviamente, per i testi a tradizione pluritestimoniale) devono affrontare nella fase di produzione e post-produzione, spesso in relazione al fatto che i ricercatori titolari del progetto sono anche editori di sé stessi. Italia (2016b, 252-3) ne ha redatto il catalogo:

Molti progetti di edizioni scientifiche digitali, una volta terminati i finanziamenti, rischiano di rimanere in uno stato di perenne 'work in progress', e di non potere quindi assicurare ai lettori l'affidabilità garantita da un prodotto della ricerca. [...] A ciò si deve aggiungere la variabilità e la deperibilità del formato di output. Un'edizione digitale progettata per essere visualizzata su un ampio schermo orizzontale verrà completamente distorta, anche nei suoi significati, dalla visualizzazione verticale offerta dai dispositivi ora prevalentemente utilizzati: tablet e smartphone. E ancora [...], anche le edizioni 'off line' pubblicate non molti anni fa in CD Rom rischiano di non essere più visualizzabili [...], 
e sono state quasi completamente sostituite dalle edizioni online. [...] Ma se il Web garantisce un rapido aggiornamento delle edizioni pubblicate, non mette al riparo dalla loro precarietà [...] quando il server che ospita l'edizione digitale non è più in grado di sostenerla, o si rompe, o l'istituzione promotrice del progetto ha cambiato direzione di ricerca, il progetto è costretto a migrare e si tocca con mano il rischio di un investimento economico dispendioso e insicuro. Il passaggio al Web, e una visualizzazione su più moderne App [...] non garantiscono maggiore affidabilità, soggette come sono le App al continuo rilascio di nuove versioni di aggiornamento.

E infine, tra i fattori che favoriscono l'edizione di testi a tradizione unitestimoniale, bisognerà annoverare gli stessi processi costitutivi di una DSE, e della sua variante specifica, la digital critical edition.

\section{SDE, o della profilassi contro il «iudicium»}

Come si è detto sopra, esiste una certa varietà di definizioni per gli oggetti in questione; una sufficientemente generale è stata proposta da Sahle $(2016,28)$ : «Scholarly digital editions are scholarly editions that are guided by a digital paradigm in their theory, method and practice». Essere 'guidati' da un digital paradigm significa che tutte le fasi dell'edizione (dalla trascrizione alla definizione del testo critico) dovrebbero essere governati da procedure informatizzate; oltre alle considerazioni di Robinson (2016), le parole di Tara Andrews (2013) non lasciano dubbi in merito:

The most immediate value of digital methods is the ability to assign as much as possible of the work - particularly that which is repetitive, exacting, and errorprone - to the computer [...]. It allows us to take advantage of the complementary strengths of man and machine to achieve a result far superior to that produced by either alone.

Automatizzare grazie ai software le procedure di trascrizione e di collazione del testo dovrebbe avere il duplice pregio di ridurre il margine di errore insito nell'applicazione umana in queste attività e di «to try to relieve what Peter Shillinburg $(1996,139)$ has called the 'idiot work' of textual editing» (Driscoll, Pierazzo 2016b, 7: gli autori si riferiscono alla fase della recensio, ma può essere estesa alle attività preliminari senza troppi rischi di forzatura semantica). Idiot work è un'etichetta poco generosa, fino alla macchietta, e tradisce un impulso riduzionistico che, tra l'altro,

si scontra inevitabilmente con il dato di fatto che emerge alla consapevolezza di ogni filologo alle sue primissime esperienze: cioè che ogni 
trascrizione, anche la più imitativa, è costretta a interpretare, come denuncia implacabilmente il riscontro con le fotografie, reso tanto più immediato proprio dal mezzo digitale. (Leonardi 2007, 67)

Perché il vero nodo della questione sta nel ruolo e nella funzione dell'interpretazione (del filologo) nell'art d'éditer (Italia 2016b, 249). Per quanto si può inferire dalla letteratura critica, i cultori della digital philology pensano alla DSE come a una sorta di 'ambiente sterile' in cui ogni azione intellettuale del filologo risulta depurata delle 'scorie' della sua soggettività. Non è un caso che si insista molto, nel caso dello scrutinio di tradizioni pluritestimoniali, sul metodo cladistico (o filogenetico) come sostituto delle procedure 'lachmanniane' per la valutazione delle varianti: come scrivono Driscoll e Pierazzo (2016b, 7), esso «is perhaps the only born-digital method available in textual scholarship, since it is based on heavy computational techniques and has arisen through interdisciplinary collaboration between textual scholars, computer scientist and bio-genetists». Ma quali sono gli esiti concreti della sua applicazione? Non ne conosco molti, che siano stati pure sottoposti a falsificazione: le edizioni (cartacee e/o elettroniche) della Commedia e del De Monarchia curate da Prue Shaw (2006 e 2010), e quella (cartacea) dei Cantari della Reina d'Oriente di Antonio Pucci per cura di William Robins (in Motta, Robins 2007); aggiungerei gli esperimenti (senza esito editoriale) condotti sull'Hêliand e sulla redazione latina L del Milione (Buzzoni, Burgio, Modena, Simion 2016). I dati ottenuti dai nostri esperimenti coincidono, nel loro significato, con quelli presentati da Ribaudo (2013) a proposito del Dante elettronico e da Trovato (2009, 91-7) sull'edizione Robins dei cantari pucciani; essi si possono riassumere nelle prime righe dell'Abstract di Ribaudo $(2013,95)$ :

I risultati dell'indagine sono concordi: la procedura informatizzata riesce al massimo a stabilire i raggruppamenti, ma non la direzione della dipendenza che si determina sempre attraverso gli errori, e spesso fornisce un quadro falsato rispetto alla realtà storica, in quanto condizionata dalle esigenze del calcolo statistico. Restano indispensabili la conoscenza della storia della tradizione e la valutazione qualitativa delle singole varianti onde evitare che l'analisi informatica travalichi il suo ruolo di supporto all'indagine filologica.

Espulsa dalla porta, l'interpretazione (e con essa la responsabilità intellettuale del filologo) rientra dalla finestra... Il metodo cladistico ha il suo fascino, ma è oneroso da applicare, e dà risultati che difficilmente 'reggono' al contro-interrogatorio. Si capisce allora perché Robinson (2016) neghi insomma che si sia ormai pienamente nell'orizzonte di una digital revolution - visto che "with rare exceptions, both old and new philology remain fundamentally non-digital in their methods, eschewing the stand- 
ardization and formal models that computers by their nature enforce» (Andrews 2013), in particolare nell'applicazione di modalità automatizzate di recensio -; e si capisce perché nella più parte delle edizioni digitali si colga un atteggiamento di relativismo intellettuale, di «rinuncia programmatica» alla scelta tra le lezioni nella mera presentazione dell'insieme delle varianti attraverso la trascrizione integrale di tutti i testimoni: l'effetto è che queste edizioni tendono ad appiattirsi sul digital archive a causa di una comune assenza di orientamento gerarchico ( «ogni attestazione risulta sullo stesso piano dell'altra, senza la possibilità di istituire relazioni o tanto meno gerarchie, cioè senza una chiave di lettura possibile per la ricostruzione della diacronia di quella data tradizione manoscritta», Leonardi 2007, 689: con rinvio, tra l'altro, a Robinson 2005 a proposito dei digital archives).

Eppure, anche al di fuori del recinto dell'ortodossia della digital revolution, sussistono le condizioni per una fruttuosa collaborazione tra applicazioni digitali e filologia ricostruttiva (e non solo new). Retrospettivamente, a pubblicazione avvenuta del Ramusio digitale, mi sono ritrovato pienamente nel disegno elaborato dalle osservazioni di Leonardi $(2007,66)$ :

Sul piano della constitutio textus, la virtualità della pagina on line, la sua natura ontologicamente non definitiva, e forse addirittura la minore fisicità del supporto, dovrebbero - avrebbero dovuto - facilitare e ancor più legittimare la tendenza a proporre ricostruzioni, a sperimentare congetture, a realizzare in forma di testo quelle ipotesi che tradizionalmente la rigidità e la dimensione univoca della carta stampata impedisce di formulare se non in nota. Inoltre, sul piano della recensio, l'incomparabile aumento di capacità e di funzionalità offerto dall'informatica alla registrazione e alla visualizzazione di numeri anche elevati di attestazioni manoscritte, per di più presentabili non solo in forma di trascrizione, ma anche con il corredo della riproduzione fotografica, dovrebbe - avrebbe dovuto - potenziare enormemente le ragioni e le modalità stesse della collazione, e quindi quella dimensione comparatistica e diacronica intrinseca al modello genealogico-stemmatico. (Leonardi 2007, 66)

Ma certo, la prospettiva indicata da Leonardi prevede che l'attività del filologo (e il suo diritto alla famigerata interpretazione) si ponga in una relazione almeno paritaria con i processi digitali ('almeno' è un eufemismo: è abbastanza chiaro che il dinamismo sotteso alla citazione è l'assunzione da parte del filologo del controllo delle operazioni), e probabilmente non si tratta della prospettiva da cui muovono le riflessioni di studiosi come Robinson o Andrews. D’altra parte, se le considerazioni che ho esposto qui (e in particolare sulle pratiche della filologia ricostruttiva) hanno un qualche fondamento, esse dovrebbero incoraggiare alla 'contaminazione' di metodi e pratiche, e non alla difesa 'purista' dei confini di una nuova disciplina umanistica. 


\section{Sul Ramusio digitale (per chiudere)}

Per chiudere, ancora Ramusio. Come ho già detto, se osservo questa edizione attraverso le lenti del dibattito intellettuale di cui, per frammenti selezionati, ho dato conto qui, devo ammettere che la sua progettazione e implementazione hanno risposto sostanzialmente al principio di metodo «On s'engage, et puis on voit!». E tuttavia mi pare che l'edizione si ponga in interlocuzione con alcuni dei problemi sollevati da questo dibattito. Procedo ancora una volta per item sommariamente sbozzati: e mi pare che tre meritino di essere sottolineati.

(1) Il Ramusio digitale non è propriamente un'edizione critica (perché l'originale' è dato, e non va ricostruito) né una édition génétique (perché non dà conto di un 'processo' di composizione, per stadi e stati diversi, di un testo): si fa carico di un testo, per dare conto del processo intellettuale che lo ha prodotto, ed è dunque animata da un'intenzione esplicitamente ricostruttiva. Sotto questo profilo non è una DSE nel senso indicato da Robinson, Andrews, ecc.: il controllo di tutte le operazioni - in tutte le sue fasi: trascrizione/edizione delle fonti, valutazione gerarchica del loro peso nella composizione dell'edizione ramusiana, pericope per pericope, riconoscimento dei processi intertestuali tra fonti e edizione - è esplicitamente e saldamente attribuito al filologo, e l'applicazione digitale è stata disegnata per aderire, e dare funzionalità, al fluire della riflessione filologica.

(2) D'altra parte, il Ramusio digitale condivide con moltissime delle edizioni citate nel repertorio di Sahle l'attenzione bédieriana ai singoli oggetti (le fonti di Ramusio), che vengono forniti al lettore nella loro integrità, per una ricezione autonoma e distinta dal testo di riferimento dell'edizione. Uno degli obiettivi dichiarati del progetto è sempre stato evitare di trasformare oggetti individui e vivi (le edizioni medievali del testo poliano note a Ramusio, e quelle che agirono come antigrafi di quelle) in parcelle manipolabili dal tritacarne del commento (cf. Burgio, Buzzoni, Ghersetti 2012).

(3) Quanto è detto sub (1) dà pure conto del ruolo giocato dalla modalità ipertestuale dell'edizione. Un buon termine di paragone è fornito da quanto scrive Sahle $(2016,19)$ :

the hyperlinks [...] restructure the contents of editions, open up new and manifold paths of reception and blur the boundaries between an edition and its contexts. The persuasive linkage between different contents and parts promote a modularized structure and a module-oriented vision of scholarly editions. Instead of concentrating on one authoritative reading as the primary goal and content, digital editions connect various forms of representation with editorial knowledge and contextual material. This 
is brought to the public in the process of a fluid publication in a double sense. What we see on the screen is often generated in real time from the current state of data, representing the current state of the editorial knowledge in a project. This is one aspect of fluidity. The other is the loss of a distinct mode of publication. Release early - release often! The edition loses its recognisability as an authoritative, final statement. Instead, it becomes a permanent but potentially always changing documentation of an ongoing examination and processing of the objects in question. In this way, the edition as a publication is a process rather than a product. [Corsivi nell'originale]

Nelle parole di Sahle aleggia un certo entusiasmo tardo-novecentesco per la dissoluzione del testo come segno monolitico dell'auctoritas dell'autore, e per la valorizzazione del process a disfavore del product, che mi pare interessante ma non realmente decisivo. In effetti, uno dei grandi vantaggi delle edizioni digitali è la loro duttilità, e la loro disponibilità a essere sottoposte a ripetuti processi di correzione (sotto questo profilo, il loro comportamento è lo stesso di qualsiasi testo pensato esclusivamente per la Rete, come ha descritto Italia 2016a - cf. supra, § 1); e anche il Ramusio digitale ha goduto di questi vantaggi: online è la seconda edizione corretta, e una nuova revisione (con l'aggiunta di nuovi materiali) è prevista entro il 2018. Ma ho qualche difficoltà a pensare tale 'emendabilità' come un tratto caratteristico di un testo fluid: in fin dei conti, il lettore nel tempo ha a disposizione un solo testo per volta, ancorato all'indicazione temporale della sua pubblicazione, e non la pluralità delle sue releases (e anzi, quello che non può fare, è recuperare la release/edizione precedente, cosa che invece il supporto cartaceo permette: paradossalmente, il processo rafforza l'unicità del testo...). Più utile è il richiamo al public. Gli hyperlinks hanno il vantaggio di lasciare libero il lettore nella definizione di un suo percorso di lettura/uso del testo. Il quale lettore, nel Ramusio digitale, può decidere di leggere il commento capitolo per capitolo, o di dedicarsi direttamente alla lettura di una singola edizione del Milione, oppure di organizzare una lettura 'a grappolo', a partire da una forma marcata qualsiasi, delle schede di commento; tutto questo genera una pluralità di letture individuali, e se si vuole estremizzare, di testi altri rispetto a quello precostituito dal filologo. Ma, appunto, non estremizzerei troppo: la sintassi ipertestuale dell'edizione è comunque chiaramente definita dal filologo prima di qualsiasi lettura, risponde alle motivazioni intellettuali che hanno motivato l'impresa, e pertanto rimane sempre e decisamente visibile nella morfologia dell'ipertesto: al lettore resta la libertà di seguirla nella sua declinazione lineare di fruizione nel tempo, o di abbandonarla seguendo l'uzzolo del momento. Qualcuno potrebbe vedere in questa l'ennesima affermazione dell'auctoritas dell'Autore e del Testo, e l'ennesima declinazione autoritaria della significazione (in questo caso travestita di libertari 
panni digital: il solito lupo travestito da agnello...). Io preferisco pensare a un compromesso ben temperato nella direzione di una 'filologia del lettore': indicare chiaramente quale Ramusio l'utente legge nel momento in cui legge Ramusio, e lasciarlo libero di usufruirne come vuole, come può. 


\title{
La variazione lessicale nel Milione Interferenza linguistica e costanti interpretative
}

\author{
Irene Reginato \\ (Università Ca’ Foscari Venezia, Italia)
}

\begin{abstract}
The study aims to demonstrate the productivity of the concept of linguistic interference in the analysis of the lexical variation in the Devisement du Monde/Milione, with particular reference to Ramusio's edition. First, linguistic interference has a textual utility and helps examine, from a different perspective, the problem of Ramusio's sources. Secondly, the analysis of lexical variation serves for methodological purposes, since it shows not only the results of Ramusio's action, but also the outcomes of linguistic interferences dating back to previous tradition. Finally, the wide variety of examples offers a pretext for more general considerations about the relationship between copy and translation, formal and content mistakes, monogenetic linguistic contact and polygenetic cognitive operations.
\end{abstract}

Sommario 1 Preliminari. - 2 Un vaso esemplare. - 3 «che si chiamano...». Prestiti lessicali. - 4 Catene di interferenza e gerarchia delle fonti. - 5 La parte di Ramusio. Errori di copia ed errori di traduzione. - 6 Fautes de sens. -7 Meccanismi cognitivi e poligenesi. - 8 Conclusioni.

Keywords Devisement du Monde. G.B. Ramusio. Linguistic interference. Lexical loans. Copying vs translating mistakes.

\section{Preliminari}

Nella definizione di Weinreich ([1953] 1974, 3), i fenomeni d'interferenza linguistica sono «quegli esempi di deviazione dalle norme dell'una e dell'altra lingua che compaiono nel discorso dei bilingui come risultato della loro familiarità con più di una lingua, cioè come risultato di un contatto linguistico». Il termine interferenza indica dunque l'azione di un sistema linguistico su un altro, o di più sistemi linguistici insieme, il cui risultato è una serie di tratti devianti e di esiti più o meno erronei rispetto alle norme della lingua d'arrivo.

Questo studio si propone di sfruttare il concetto d'interferenza linguistica per dimostrarne la produttività nell'analisi della variazione lessicale nel Milione, con particolare riferimento alla redazione ramusiana. Anzitutto, produttività a fini testuali, poiché gli esiti dell'interferenza linguistica consentono di aggredire, sotto un'altra prospettiva, il problema filologico dell'individuazione delle fonti di Ramusio. In secondo luogo, produttività a fini metodologici, poiché la varietà della campionatura scelta permette 
di illuminare molteplici livelli e modalità di azione dell'interferenza, modulando e arricchendo di sfumature la definizione citata in apertura. Infine, produttività nella comprensione del modus operandi dell'intellettuale veneto, delle sue intenzioni di editore e della sua personale idea del Milione.

Prima di passare in rassegna i casi d'interferenza utili alla dimostrazione, è opportuno sviluppare due premesse teoriche sul nostro oggetto di studio. La prima riguarda la sua natura di testo scritto. Gli studi di Weinreich e il concetto d'interferenza si collocano all'interno di una riflessione relativa ai parlanti bilingui, e tipica di una comunicazione orale nella quale le due o più lingue intrattengono un contatto di tipo sincronico. Non così nell'indagine dell'interferenza applicata al nostro testo, dove il contatto linguistico si manifesta nell'operazione scritta della traduzione, e si colloca sull'asse diacronico della trasmissione testuale. Eppure, l'analogia delle dinamiche messe in gioco autorizza l'estensione metodologica del concetto d'interferenza, come suggeriva già Benvenuto Terracini ([1957] 1983, 17) scrivendo che il problema della traduzione «si lascia impostare come un problema di bilinguismo».

La seconda premessa muove esattamente dalla riflessione su questa diacronia multilingue. L'operazione editoriale e traduttoria di Ramusio non è che l'ultimo movimento di un percorso testuale complesso nel quale trasmissione diretta e indiretta si affiancano e si compenetrano in un rapporto osmotico ove «i confini tra copia e traduzione sono labilissimi» (Bertolucci Pizzorusso 1975, 351). Così, i casi d'interferenza riscontrabili nell'edizione ramusiana sono spesso l'esito non di un contatto linguistico, ma di più contatti successivi, accumulatisi e conservatisi nello scorrere del testo nel tempo. L'analisi della variazione lessicale nei Viaggi permetterà allora di distinguere, caso per caso, il risultato dell'azione individuale - più o meno consapevole - di Ramusio dagli esiti 'ereditati' dalla tradizione a lui precedente. L'indagine sull'origine e la natura delle lezioni considerate sarà infine il pretesto per considerazioni di portata più generale sulla dialettica tra attività di copia e attività di traduzione, sulla differenza tra lezioni solo formalmente 'devianti' e vere e proprie fautes de sens, e sulla distinzione tra meccanismi rivelatori di un contatto linguistico preciso e operazioni cognitive universali e poligenetiche.

Per tutti i casi citati, il primo riferimento bibliografico sono le voci corrispondenti all'interno del «Lemmario» che correda l'edizione digitale dei Viaggi di Messer Marco Polo.

\section{Un vaso esemplare}

Può essere utile illustrare un esempio particolarmente denso, dal quale partire per individuare i numerosi fili che compongono l'intricato fenomeno dell'interferenza nel testo in esame. Nel decimo capitolo del secondo 
libro, dedicato alla descrizione dei sontuosi banchetti alla corte mongola, la redazione ramusiana s'attarda su giganteschi vasi porta-bevande dai quali ciascuna coppia di convitati attinge per mezzo di coppe dorate:

Et in detto scrigno stanno tutti i vasi del signore, co' quali si porge da bere, et sonvi alcuni d'oro bellissimi, che si chiamano 'vernique', le quali sono di tanta capacità che ciascuna, piena di vino o vero d'altra bevanda, sarebbe a bastanza da bere per otto o dieci huomini; et a ogni due persone che seggono a tavola si pone una verniqua piena di vino con una 'obba', et le obbe sono fatte a modo di tazze d'oro che hanno il manico, con le quali cavano il vino dalla verniqua, et con quelle bevono, la qual cosa si fa cosí alle donne come alli huomini. (R II 10 7)

Le lezioni vernique/verniqua si presentano come due forme (plurale e singolare) di uno stesso termine che non trova ulteriori attestazioni in italiano e che si qualifica dunque come un hapax attribuibile a Ramusio. Più che d'invenzione originale, tuttavia, si tratta qui di un prestito lessicale che ritroviamo nella versione franco-italiana F:

Se trait le vin, ou le chier bevrajes que hi soit, et s'en enplent grant vernique d'or qe bien sunt tiel qe tienent tant vin que .VIII. homes ou .X. en av<r>oient assez, et se metent, entres deus homes que sieent a table, un; et chascun de cesti deus homes hont une coppe d'or a maneque, et con celle cope prennent dou vin de cel grant vernique d'or. Et ausint en ont entre deus dames: un de celz grant et deus coupes, comant ont les homes. (F LXXXV 12-13)

Come si sa, per prestito lessicale s'intende un termine (o più d'uno in caso di una locuzione o costruzione sintattica) che una lingua preleva da un'altra, accogliendolo nel proprio repertorio lessicale. Nel nostro caso specifico, ovvero entro un meccanismo di traduzione di testi scritti, possiamo definire prestito il risultato di un'imitazione pedissequa del testo

1 I corsivi, da qui in poi, sono sempre aggiunti. Le sigle citate corrispondono ciascuna a una delle redazioni dell'opera poliana. R rinvia ai Viaggi di messer Marco Polo di Ramusio (Simion, Burgio 2015); F indica la versione franco-italiana del ms. BnF fr. 1116 (Eusebi [2010] 2018), mentre è detta Fr la versione francese trecentesca (Ménard 2001-09). TA è la versione toscana edita da Bertolucci Pizzorusso (1975), VA quella veneto-emiliana (Barbieri, Andreose 1999) e VB è la versione veneziana oggetto della tesi di Gennari (2008-09). Per le versioni latine: $\mathrm{Z}$ indica la versione del ms. Zelada 20 dell'Archivio Capitolare di Toledo (Barbieri 1998); P quella di fra' Pipino da Bologna; L un'epitome latina prossimamente edita da Burgio (in corso di stampa). Le versioni F, L, P, V, VA, VB, Z e R sono tutte leggibili in Simion, Burgio 2015. Per tutti i termini commentati in questo saggio, si rimanda alla voce corrispondente del «Lemmario» in Simion, Burgio 2015. Nel caso di vernique, la voce del «Lemmario» è curata da Andreose e Burgio. 
di partenza, che produce il trasferimento di un termine da quest'ultimo al testo di arrivo. Comunque sia, il termine nuovo può essere accolto tal quale e in modo immediato, oppure può subire modifiche più o meno vistose di tipo grafico-fonetico, tali da 'acclimatarlo' alle convenzioni della lingua d'arrivo.

Il caso di vernique/verniqua è particolarmente interessante in questo senso, poiché il lemma subisce un'ortopedizzazione italianizzante solo parziale e, nella prima occorrenza, di fatto nulla. La conservazione della forma francese vernique (un plurale 'pronto all'uso' data la mancanza di $-s$ finale) e, dato valido anche per la variante singolare verniqua, la resistenza del digramma 〈qu> (elemento, questo, ricavabile solo poiché lavoriamo su testi scritti), consentono di introdurre il primo dei problemi sopra annunciati, ovvero l'esistenza di casi d'interferenza in diacronia e su più livelli successivi. Se infatti qualifichiamo vernique come un prestito che Ramusio ricavò da una delle sue fonti, ne ricaviamo necessariamente che la fonte in questione doveva anch'essa presentare il lemma in questa stessa forma francese. Il termine vernique è dunque l'esito di un contatto linguistico anteriore all'attività traduttoria dell'umanista, e qualificabile come prestito già all'altezza del suo modello.

La ricostruzione dell'interferenza lessicale in diacronia consente di ripercorrere à rebours la storia linguistica del testo, e di dimostrare la valenza che questo tipo d'indagine può avere anche sul piano genealogico e, in particolare, circa il problema dell'individuazione delle fonti di Ramusio. Una prima osservazione porta sul contenuto del passo in esame. Il prestito vernique svolge nel concreto la stessa funzione che, sul piano ecdotico, hanno le lezioni comuni: la sua presenza, in altre parole, consente di escludere la derivazione del testo ramusiano da un modello nel quale tale lezione non è presente. Gli studi riuniti in Burgio (2011) e in Simion, Burgio 2015 hanno dimostrato che sul tavolo da lavoro di Ramusio si incontravano almeno tre versioni del racconto poliano: (1) un esemplare della redazione P di proprietà dello stesso Ramusio; (2) un prezioso codice oggi perduto appartenente alla famiglia Ghisi e facente parte della stessa 'costellazione' Z del ms. di Toledo, ma certamente più completo di questo; (3) un esemplare del rimaneggiamento veneziano VB. Ora, di queste fonti, la sola a contenere il passo in esame è la latina $\mathrm{P}$, mentre l'intero segmento è omesso sia nella redazione VB sia nella versione $\mathrm{Z}$ che, perduto il Ghisi, leggiamo nel manoscritto toledano. Il passo di Pipino, tuttavia, non presenta il lemma vernique, sostituito con un più generico «vas aureum»:

In medio vero aule regie quoddam vas aureum ponitur vino plenum vel pretiosa alia potacione unius vegetis seu dolii vel currus mensuram capiens, iuxta quod sunt hinc inde quatuor dolia magna de auro purissimo minora aliquantulum vase illo in quo vinum defluit de vase maiori, de quibus vasis hauritur vinum in urceos aureos qui inter duos ponuntur 
in mensis cunctorum discunbentium in regali convivio, quorum quilibet est tante magnitudinis ut vinum pro octo vel decem capiat hominibus; quilibet etiam in magno aureo cipho bibit habente pede $\langle\mathrm{m}>$ et stipitem aureum. Sunt universa hec vasa valoris maximi. (P II 13 4-5)

Assente in $\mathrm{P}$ e in $\mathrm{VB}$, non confermata dallo $\mathrm{Z}$ toledano ma presente in $\mathrm{F}$, la lezione vernique risale dunque necessariamente allo $\mathrm{Z}$ Ghisi e consente quindi di riconoscere nel famoso e perduto manoscritto la fonte di Ramusio per la pericope in questione. Non solo, la forma francese del lemma prova anche che il modello dello Z Ghisi era un testo franco-italiano vicinissimo a $\mathrm{F}$, dal quale l'anonimo redattore lesse la forma vernique e la riprese 'pigramente' nella sua traduzione.

Quest'inerzia nella (non) traduzione di vernique apre la riflessione su un altro degli aspetti dell'interferenza sopra menzionati, relativo al grado di consapevolezza che interviene in un fenomeno di contatto linguistico. È evidente che, se 'italianizza' vernique in verniqua, adeguandosi alla morfologia italiana nel passaggio 'plurale-singolare', Ramusio commette un errore di distrazione dimenticando di ortopedizzare i due termini anche a livello grafico-fonetico, e di trasformare i digrammi 'francesi' que/qua negli 'italiani' che/ca. Ė proprio questa svista, questa momentanea défaillance dell'attività traduttoria, che consente il prodursi di una forma deviante e rivelatoria di un fenomeno d'interferenza. La stessa svista, tuttavia, coinvolse prima di Ramusio anche il redattore di $\mathrm{Z}$ (almeno lo $\mathrm{Z}$ del codice Ghisi), e invita a qualche considerazione ulteriore.

Il termine vernique è attestato solo nella versione $\mathrm{F}$ e si presenta come un hapax nel lessico antico francese, la cui etimologia è da collegarsi a quella di vernis (fr.)/vernice (it.). Caso tipico di creazione onomastica nel quale un oggetto assume il nome della città alla quale è legato, vernis deriva molto probabilmente - per il tramite italiano vernice (cf. FEW, 1, 332-3) - da Berenice della Trogloditica, ${ }^{2}$ città sul mar Rosso nel confine tra Egitto e Sudan, storicamente nota per la produzione della sandracca, resina derivata da una particolare pianta diffusa nell'Africa settentrionale (una conifera o - secondo Ineichen - il ginepro, cf. LEI, V, s.v. «Berenice»). In testi latini medievali, il termine è attestato nelle forme vernix, vernice, vernisse (LEI) ma anche veronice (FEW); in volgare italiano, esso si diffonde da Bergamo (dove si attesta nella forma vernis) alla Sicilia (vernici), passando per il padovano vernixe del Libro agregà de Serapiom (LEI). La presenza, allora, dell'occlusiva -ique nella forma poliana vernique potrebbe far pensare a una forma greca del termine circolante nel francese levantino e appresa da Marco nel suo 'apprendistato' umano ma anche linguistico

2 La precisazione è volta a distinguerla da Berenice in Cirenaica, corrispondente alla libica Bengasi. 
in Outremer. ${ }^{3}$ In ogni caso, interessa notare che né vernis né vernique avrebbero propriamente il significato di 'vaso', un passaggio metonimico attuato esplicitamente da $\mathrm{P}$ - che scrive «vas aureum» - ma anche dalla versione Fr, che sostituisce vernique con vernigal, «grande coupe vernie» (Ménard 2001-9, 3: 34). ${ }^{4}$

Tornando al nostro ragionamento, la mancata traduzione di vernique da parte del redattore di Z (quantomeno dello Z Ghisi consultato dal Ramusio) può essere facilmente spiegata come il risultato della non comprensione di una forma di fatto eccentrica del testo di partenza. Una difficoltà, insomma, di traduzione. Eppure, il termine non viene né eliminato né sostituito da uno equivalente, e questo perché il redattore lo interpreta come un appellativo

3 «Les voyages de Marco - scrive Zinelli $(2016,229)$ - sont à l'origine de ses connaissances», tanto che il lessico poliano si caratterizza per una «dimension méditerranéenne plus qu'italienne» e, per quel che concerne i prestiti, per «un lexique des realia dans la plupart des cas déjà francisé et monnaie courante dans les ports et sur les marchés» (Zinelli 2016, 220 e 227). Come grecismo, vernique non sarebbe un caso isolato: i contatti correnti e documentati tra greco, veneziano e francese levantino soprattutto a Cipro e Acri sono all'origine di una serie di grecismi passati poi - con o senza un tramite italiano - alla koiné del francese d'Outremer, basti pensare a gondole, probabile grecismo passato al genovese tramite il veneziano, e poi al francese levantino (cf. Zinelli 2016, in particolare 211, 215 e 217). A livello fonetico, il passag-

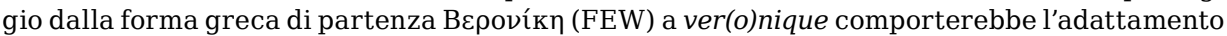
'standard' della velare к all'occlusiva $c$ e della vocale finale atona a $e$ muta (Minervini 2012, 61-2). Assai poco probabile, invece, è che vernique rappresenti una goffa 'francesizzazione' di vernice da parte dei co-autori Marco e Rustichello: anzitutto, F riserva l'uscita in -ique a lemmi il cui corrispondente italiano presenta già un esito velare, (generalmente in -co: ondanique per 'ondanico', diabolique per 'diabolico' e il curioso carique per 'carico' in F CXCI 3); inoltre, una forma foneticamente affine quale pernice dà perdrix o perdrices (F LXXIII, 24 e 32); infine, il passaggio al francese comporta semmai la palatalizzazione di forme velari, e non viceversa (si pensi a ostrice 'ostrica' in F CLXXIII 9). Per una discussione più ampia sull'origine della forma e sulla presenza di grecismi in F, si rimanda a Andreose 2018. Si noti come il FEW $(1,335)$ attesti anche l'etimo bretone bernic, indicante la 'patella' (in tedesco Entenmuschel), ovvero una particolare specie di mollusco caratterizzato da un guscio a forma di cono appiattito (donde l'etimo latino patella, 'padella, piatto', diminutivo di patĕra).

4 Generalmente accostata alla forma veneziana vernegal come «catino di legno in cui si pone la minestra ecc. destinata per cadaun pasto dell'equipaggio sul mare» (Boerio 1867), più che «terme vénitien» (Ménard 2001-9, 3: 34), la forma vernigal andrebbe qualificata come mot méditerranéen, ovvero come lemma «que le français emprunta aux autres langues romanes dans le domaine de la navigation et du commerce» (Zinelli 2016, 213). In questo senso, essa è parallela al genovese vernigale, ed è attestata in un altro testo scritto nell'Oriente latino, la Règle de l'Ordre du Temple (De Curzon 1886, 214, citato in Godefroy 1881, s.v.). Una variante concorrente è poi vernicato, aggettivo verbale sostantivato dal verbo vernicare (inverniciare), che il LEI definisce 'scodella, catinella, recipiente' e attesta in Ciriffo Calvaneo (XV sec.) e nel Milione toscano («Havvi vasella e vernicati d'oro» nell'edizione Segre, Marti 1959, 358; si veda però l'edizione di Bertolucci Pizzorusso $(1975,132)$, dove il termine è aggettivo: «[Àvi] vasegli vernicati d'oro»), segnalandone anche varianti napoletane (vernecato) e siciliane (virnicatu). La qualità 'mediterranea' del termine è poi confermata dalla sua diffusione in castigliano (bernegal) ma soprattutto in catalano, sia nella variante participiale vernigat/vernegat (Alcover i Sureda, de Borja Moll 1962) sia in quella in -al (un vernigal è attestato in Bolòs, Sànchez-Boira 2014, 1533). Per origine e diffusione di vernegal, cf. anche Vidos (1939, 604-8) e Fennis (1995, 361, s.v. «bernigal»), che propone lo stesso etimo greco berenike. 
originario di un oggetto esotico - una gigantesca coppa laccata - privo di corrispondenti familiari. Alla base del prestito, quindi, va posta una situazione di vuoto espressivo che l'adozione del termine ignoto ha la funzione di colmare. Il ragionamento trova una conferma concreta nel testo di Ramusio. Rispetto alla frase di F - «Se trait le vin, ou le chier bevrajes que hi soit, et s'en enplent grant vernique d'or» - Ramusio inserisce una proposizione relativa - «che si chiamano» - che costituisce una tipica formula di introduzione a una realtà ignota: «Et in detto scrigno stanno tutti i vasi del signore, co' quali si porge da bere, et sonvi alcuni d'oro bellissimi, che si chiamano 'vernique'» (corsivo aggiunto). L'identificazione di vernique come denominazione propria di un oggetto esotico facilita, quindi, in Ramusio, una maggiore passività nell'atto di traduzione e una minor consapevolezza nell'accoglimento del prestito, lasciato tal quale o - quando il senso lo richiede - solo debolmente acclimatato nel singolare verniqua.

Il complesso caso ora analizzato fornisce le piste che seguiranno i paragrafi successivi di questo studio, ciascuno approfondendo, attraverso l'analisi di esempi concreti, gli aspetti finora passati in rassegna.

\section{3 «che si chiamano...». Prestiti lessicali}

Molti dei prestiti lessicali presenti nella tradizione del Devisement du Monde rappresentano, com'è facile immaginare, appellativi specifici destinati a oggetti o luoghi strettamente collegati alla realtà alla quale appartengono. Mutuati tal quali da una lingua altra, essi appaiono come etichette atte a designare realia inesistenti nell'orizzonte cognitivo della lingua di arrivo, e dunque privi sia di un equivalente concettuale, sia di un item lessicale che li identifichi. In mancanza di un corrispettivo linguistico disponibile all'uso, il traduttore ricorre necessariamente al prestito, operando un trasferimento non solo di significanti ma anche di significati.

Nel capitolo relativo alla provincia di Mosul, ad esempio, Marco Polo riferisce alcune confuse informazioni sui culti cristiani orientali, tra le quali la presenza di un patriarca comune a nestoriani, giacobiti ed armeni, chiamato iacolit:

Moxul è una provincia nella qual habitano molte sorti di genti, una delle quali adorano Macometto, et chiamansi Arabi; l'altra osserva la fede christiana, non però secondo che comanda la Chiesa, perché falla in molte cose, et sono nestorini, iacopiti et Armeni; et hanno un patriarcha che chiamano 'iacolit', il qual ordena arcivescovi, vescovi et abbati. (R I 16$)^{5}$

5 Per la voce iacolit e un excursus sulla difficoltà classificatoria di Polo in ambito religioso, cf. nel «Lemmario» la voce a cura di Eugenio Burgio in Simion, Burgio 2015. 
La forma iacolit, designante una carica religiosa sconosciuta al cristianesimo occidentale, non trova corrispondenza in questo loco testuale né nella versione di Pipino, né in VB né nello $\mathrm{Z}$ toledano, che legge solamente «[...] patriarcam habent qui archiepiscopos, episcopos et abbates statuit [...]» (Z 5 4). Ancora una volta, però, l'autenticità della lezione è garantita dalla versione di F XXIII 4: «[...] il ont patriarche, ke l'apelent Iatolic, et cestui patriarche fait arcevescheve et vescheve et abés [...]» (corsivi aggiunto). La forma che il lemma assume nel testo franco-italiano, inoltre, conferma quanto detto a proposito dei prestiti lessicali: in assenza di un termine equivalente nella lingua d'arrivo, il traduttore di fatto non traduce e mantiene il lemma nella sua forma originale o quasi (lo scambio $t / c$ è naturalmente irrilevante in questo senso). Così, l'anonimo redattore del codice Ghisi non tradusse in latino il termine presente nel suo modello franco-italiano, e allo stesso modo si comportò Ramusio. Come per vernique/verniqua, inoltre, si può osservare che il prestito è preceduto dalla relativa che (si) chiamano..., stringa che agisce come una sorta di meccanismo di sospensione dell'attività traduttoria, attivando l'accoglimento passivo del termine straniero.

È quanto accade anche nel caso seguente, la cui analisi offre lo spunto per considerazioni di metodo dal punto di vista dell'ipotesi genealogica. Nella provincia di Zorzania (Georgia), Marco Polo racconta che «[...] si lavorano panni di seda et d'oro, et vi sono astori nobilissimi, che si chiamano 'avigi'» (R I 5 9) (corsivi aggiunti). ${ }^{6}$ L'informazione relativa agli splendidi astori georgiani, caratterizzata dalla consueta sequenza 'verbo chiamar(si) + appellativo indigeno', è conservata tal quale nella sola versione $\mathrm{L}$, che legge «Inveniuntur eciam ibi optimi austures dicti 'avigi'» (L 20 6) (corsivi aggiunti). La convergenza R-L non smentisce, in questo caso, la comprovata partecipazione anche di questa redazione latina all'editio variorum ramusiana. Tuttavia, il lavoro di collazione eseguito preliminarmente all'edizione digitale ha sì rilevato apporti di L, ma ne ha allo stesso tempo evidenziato la portata minima e quasi trascurabile se paragonata a quella di P e, soprattutto, di Z (cf. Simion, Burgio 2015). In alcuni casi, infatti, l'evidenza di un apporto di L è messa in discussione dal ragionevole dubbio che la lezione in questione fosse presente anche a livello dell'archetipo di $\mathrm{Z}$, e che Ramusio possa dunque averla derivata dal famoso codice Ghisi. L'ipotesi, che ha il vantaggio di una maggiore economia sul piano logico, è supportata in questo caso specifico dalla lezione di V. Questa redazione veneta, la cui partecipazione al patchwork ramusiano non è stata confermata, presenta al capitolo 12 la lezione seguente: «et lì se lavora de beli drapi de seda ed oro, li mior del mondo, et vien chiamadi 'ostori'» (corsivi aggiunti). Il passo, per quanto corrotto, consente di riconoscere la solita stringa introduttiva all'appellativo indigeno («vien chiamadi»), e induce a

6 Per avigi, cf. Pelliot 1959-73, 57-8 nota 41. 
pensare che il termine avigi si collocasse più in alto di L, donde la traccia in $\mathrm{V}$. L'apporto della redazione latina $\mathrm{L}$ appare dunque ridimensionato e la conclusione più economica (e dunque preferibile) è che Ramusio abbia tratto avigi dallo Z Ghisi anziché da L, e che da un antigrafo analogo a Z derivino anche la lezione di $\mathrm{L}$ e l'errore di $\mathrm{V}$.

Una precisazione. L'adozione del termine indigeno sotto forma di prestito non esaurisce le possibilità di reazione linguistica all'ignoto, e spesso il vacuum lessicale è colmato con denominazioni nuove $\mathrm{o}$, in modo più immediato e frequente, con formazioni analogiche. "L'homo viator - scrive Folena- «è sempre un animal symbolicum, e analogicum»: attraverso l'analogia, egli designa una realtà ignota per mezzo di un referente noto, «facendo conoscere il dissimile col simile» (Folena 1991, 103). Nella sua forma più completa, il procedimento analogico specifica la nuova denominazione con l'aggiunta di tratti distintivi - un aggettivo o un complemento d'origine - che differenziano l'oggetto esotico rispetto al familiare, rimarcando l'alterità nella similarità. Confrontato ai frutti della palma da cocco, quindi, il viaggiatore li accosta a gigantesche noci e le differenzia da quelle conosciute in Europa attraverso l'aggiunta della determinazione geografica d'India:

Trovasi ancho noci d'India, grosse come è il capo dell'huomo, le quali sono buone da mangiar, dolci et saporite et bianche come latte, et il mezzo della carnosità di detta noce è pieno di un liquore come acqua chiara et fresca, et di sapor miglior et piú delicato che 'l vino o vero di alcuna altra bevanda che mai si bevesse. (R III 13 8) ${ }^{7}$

Nella forma più sintetica, invece, l'aggiunta di una determinazione specifica salta, e la realtà esotica nuova è designata per mezzo del solo termine familiare, in una sorta - e sono ancora le parole di Folena - di metafora referenziale (cf. Folena 1971-3, 192). Così, confrontandosi con le bevande alcoliche asiatiche a base di riso, zucchero e datteri, Marco Polo le chiama vino:

Sono quivi molti arbori di palme, che fanno buoni dattali in abbondanza; non vi nascono biave, se non risi et miglio, et bisogna che vi siano condutte delle biave di altre regioni. Non hanno vino di uva, ma lo fanno di risi, zucchero et dattali, ch'è delicato a bevere [...]. (R III 41 6)

Esempi di questo genere abbondano nel Milione ma, proprio poiché sfruttano termini già presenti nella lingua d'arrivo, si rivelano di fatto molto meno significativi da un punto di vista dell'interferenza linguistica che da un punto di vista culturale, e non saranno dunque trattati in queste pagine.

7 Sulle noci d'India, si veda nel Lemmario la voce di A. Ghersetti in Simion, Burgio 2015. 


\section{Catene di interferenza e gerarchia delle fonti}

Casi come vernique/verniqua mostrano che la variazione lessicale nel testo di Ramusio è talvolta l'esito ultimo di fenomeni d'interferenza collocati più in alto nella catena testuale. All'interno dei casi d'interferenza rinvenibili in $\mathrm{R}$, dunque, ve ne sono alcuni che risalgono ai suoi modelli, essi stessi delle traduzioni e quindi il risultato di una trasmissione testuale indiretta lungo l'asse diacronico. In alcuni casi occorre risalire di più di un passaggio per spiegare la genesi dell'errore, come accade nell'esempio seguente.

Nel capitolo II 16 - mancante sia in VB sia nello $\mathrm{Z}$ toledano - $\mathrm{R}$ descrive i padiglioni di caccia di Qubilai. Al paragrafo 17, in particolare, informa che dalla parte interna le tende «sono fodrate et coperte di pelli armelline et zebelline» molto costose, tanto che «vale duoimila bisanti d'oro se la è perfetta, ma se ella è commune ne vale mille»; il comma si chiude con un'ulteriore nota sul valore del pellame e con la menzione di un misterioso animale non più grosso di una faina, «et li Tartari la chiamano regina delle pelli, et gl'animali si chiamano 'rondes', della grandezza d'una fuina» (corsivi aggiunti): ${ }^{8}$

Et detti padiglioni et tende di fuori sono coperte di pelli di leoni, et vergate di verghe bianche, nere et rosse, et cosí ben ordinate che né vento né pioggia li può nuocere; et dalla parte di dentro sono fodrate et coperte di pelli armelline et zebelline, che sono le pelli di maggior valuta di qualunche altra pelle, perché la pelle zibellina, se la è tanta che sia a bastanza per un paro di veste, vale duoimila bisanti d'oro se la è perfetta, ma se ella è commune ne vale mille; et li Tartari la chiamano regina delle pelli, et gl'animali si chiamano 'rondes', della grandezza d'una fuina.

Così presentato, il termine rondes appare come l'appellativo esotico di un animale tipico dei territori percorsi da Marco, e privo di corrispettivi in Occidente. A facilitare quest'interpretazione, si noti, è ancora una volta la stringa «si chiamano...». Da un punto di vista delle fonti di Ramusio, rondes è mutuato dalla redazione $\mathrm{P}$, che al comma 5 del capitolo II 20 presenta l'animale allo stesso modo, «Animalia illa a quibus hec pelles habentur dicuntur 'rondes' et sunt magnitudinis unius faine»:

Tanta enim quantitas pellium çambellinorum, quanta pro integra militis veste sufficeret, ascendit ad valorem duorum milium bisantium aureorum, si de pelle perfecta est; si autem de communi, ascendit ad valorem mille bisancium. Animalia illa a quibus hec pelles habentur dicuntur 'rondes' et sunt magnitudinis unius faine.

8 Cf. la voce di Eugenio Burgio nel «Lemmario» in Simion, Burgio 2015. 
A sua volta, Pipino trasse rondes dal suo modello, un testimone della redazione VA la cui lezione è «Li Tartari l'apelano in soa lingua rondes et sono de grandeza de fuine» (VA LXXVI 20) (corsivi aggiunti). Zoonimo esotico preceduto dalla solita stringa «si chiamano...»/《dicuntur...», con la variante attiva «Li Tartari l'apelano», rondes rimane identico nella forma attraverso almeno tre testi successivi.

Tuttavia, dal punto di vista delle conoscenze sulla fauna asiatica, a nulla giova questa resistente conservatività. Il presunto zoonimo, infatti, non corrisponde a nessun animale realmente esistente, e risulta da una corruzione del testo di F (XCIII 28) dove non v'è alcuna menzione della bestiola. Il paragrafo, infatti, termina con l'apprezzamento sul valore delle pelli d'ermellino e zibellino, e a esse attribuisce la grandezza di una faina:

Et dedens sunt toutes d'armines et de jerbelin: ce sunt andeus les plus belles pennes et les plus riches et de greingnor vailance que pennes que soient, mes bien est il voir que la pelle de gebbeline, tant qe soit a une robe d'ome, vaut bien la fin .II ${ }^{\mathrm{m}}$. beçant d'or, mes les comunel vaut .M. beçant; et l'apellent les Tartarz le[s] roi des pelames, et sunt de la grant d'une faÿne.

Come dimostra Valeria Bertolucci Pizzorusso a partire da un analogo errore nella redazione toscana $\mathrm{TA}^{9}{ }^{9}$ rondes va interpretato come l'esito della cattiva lettura di una lezione simile a «et l'apellent les Tartarz le[s] roi des pelames», dove roi des produsse verosimilmente roides e rondes. L'errore risale a VA e si trasmette tal quale a P: entrambe le redazioni, infatti, omettono la designazione roi des pelames e la sostituiscono con la menzione dell'animale. Le cose sono un po' diverse, però, in Ramusio. Edizione variorum, R presenta entrambe le lezioni, evidentemente derivate da due modelli differenti: la prima - «et li Tartari la chiamano regina delle pelli» dal suo esemplare $\mathrm{Z}$, la seconda - «et gl'animali si chiamano 'rondes'» - da Pipino.

Il caso di rondes, quindi, si dimostra significativo anche per formulare alcune considerazioni sul trattamento che Ramusio riservava alle sue fonti e sugli intenti che animarono la sua operazione editoriale. Come ha notato Mascherpa (2011, 66), l'epistola dedicatoria a Geronimo Fracastoro che apre l'edizione ramusiana informa immediatamente il lettore della gerarchia che Ramusio attribuiva ai propri modelli. Affermato che il testo originale fu scritto in lingua latina «col mezzo d'un gentilhuomo genovese», Ramusio riferisce di aver avuto a disposizione la preziosa copia dei Ghisi e di averla utilizzata per confrontarne il testo «con questa che al presente

9 Cf. Bertolucci Pizzorusso ([1994] 2011b, 119-20). TA 9326 legge «[...] e chiàmalle li Tartari le roi de pelame [...]», da un originario leroide pelame copiato senza essere capito (cf. Bertolucci Pizzorusso 1975, 147). 
mandiamo in luce», ovvero con la sua copia del Milione, un esemplare della versione $\mathrm{P}$ :

Hor trovandosi in questo stato messer Marco, et vedendo il gran desiderio ch'ognun havea d'intendere le cose del paese del Cataio et del Gran Cane [...] col mezzo d'un gentilhuomo genovese molto suo amico [...] scrisse per gratificarlo il presente libro in lingua latina, sí come accostumano li Genovesi in maggior parte fino hoggi di scrivere le loro facende, non possendo con la penna esprimere la loro pronuncia naturale. [...] Una copia di tal libro, scritta la prima volta latinamente, di maravigliosa antichità, e forse copiata dallo originale di mano di esso messer Marco, molte volte ho veduta et incontrata con questa, che al presente mandiamo in luce, accomodatami da un gentilhuomo di questa città da Ca' Ghisi, molto mio amico, che l'havea appresso di sé et la tenea molto chara. ${ }^{10}$

La gerarchia 'Ghisi-Pipino' è ribadita - nota sempre Mascherpa (2011, 66) - anche nell'ordine dei due proemi che Ramusio inserisce in apertura ai Viaggi, dove il proemio del codice Ghisi precede quello di Pipino, autorizzando a mettere in discussione la convinzione di Benedetto che l'editio ramusiana sia «nella sostanza una versione di $\mathrm{P}$ » (Benedetto 1928, CLVIII).

Tuttavia, la coesistenza dei due proemi mostra anche la volontà di Ramusio di offrire al suo pubblico di lettori un testo più completo ed esaustivo possibile - «perfettamente corretto et di gran lunga molto piú fidele di quello che fin hora si è letto» - e nel quale la riconosciuta superiorità di una fonte non ostacolasse più o meno sistematici travasi da altre versioni. Questo scrupolo di esaustività coinvolge anche la lettera del testo: Ramusio non solo corregge le sue fonti l'una con l'altra, ma le completa e talvolta duplica l'informazione. Così, descrivendo le donne di Zanzibar, che hanno nella versione di Pipino mani grandissime - «manus vero grossiores» (P III 415 ) - e in Z seni prosperosi - «sunt grosse mamas quadruplo plus aliis mulieribus» (Z 125 21) - Ramusio scrive: «Le femmine similmente sono brutte, la bocca grande, il naso grosso et gli occhi, ma le mani sono fuor di misura grosse, le tette grossissime» (R III 37 6).

Lo stesso avviene nel caso di rondes, dove non solo è evidente la volontà di completare le fonti l'una con l'altra, ma emerge anche quanto la gerarchia 'Ghisi-Pipino' sia comunque sempre passibile di momentanee e intermittenti inversioni. È stato notato infatti come, nonostante abbia disposizione un testimone particolarmente autorevole, Ramusio non si faccia scrupoli a mutuare lezioni anche bizzarre dalla redazione di Pipino, accordando a quest'ultima una fiducia talvolta acritica, dovuta verosimilmente al prestigio della redazione, alla sua circolazione in ambienti colti e, 
forse, anche a una componente affettiva verso l'esemplare in suo possesso (cf. Mascherpa 2011, 71; Barbieri 2011, 15-16; il contributo di Andreose al Libro II in Simion, Burgio 2015). Così, esattamente come per le mani delle donne di Zanzibar, Ramusio accoglie la lezione rondes come una lezione aggiuntiva la cui autenticità è garantita dalla versione pipiniana, e il cui gusto esotico impreziosisce la sua personale edizione.

Un caso simile di 'aderenza lessicale' alla versione di Pipino riguarda la denominazione di un altro oggetto sconosciuto: le slitte. Nella descrizione della Tundra siberiana, al capitolo R III 44 13, si legge che:

Et per esser i ghiacci grandi hanno fatto una sorte di carri, che quelli che habitano appresso di noi sopra monti aspri et inaccessibili li sogliono usare, et si chiamano 'tragule', che sono senza ruote, piani nel fondi, et si vengono alzando dalli capi a modo di un semicirculo, et scorrono per sopra la ghiaccia facilmente. (R III 44 13)

Il passo, che costituisce la prima menzione delle 'slitte' in area medievale occidentale, è stato studiato in Burgio 2008, ${ }^{11}$ da cui si traggono le osservazioni seguenti. La pericope ramusiana è mutuata da Pipino, e ne è prova la presenza del termine tragule, lemma volgare con il quale Pipino glossa il latino classico traha:

Hii autem canes assueti et docti sunt trahere trahas que vulgariter in Ytalia dicuntur 'tragule'; est autem traha seu tragula vehiculum sine rotis, quo apud nos montium habitatores utuntur; ad unam igitur tragulam sex canes ligant ordine congruo; tragule autem supponuntur pelles ursorum supra quas homines duo sedent: negotiator scilicet qui pro pellibus vadit et auriga qui canes regit et dirigit et viam optime novit. Quia igitur hoc vehiculum est de ligno levissimo et subtus est planum et politum et canes fortes sunt ad huiusmodi officium assueti, nec magna honera vehiculo imponunt; canes illi per lutum illud faciliter satis trahunt, nec multum in lutum figitur tragula in huius tractu. (P III 48 5)

La forma che il termine assume in F, invece, è trejes, forma che - seguendo la ricostruzione di Burgio (2008, 49-50) - è possibile interpretare come un italianismo a partire da treggia, voce regionale indicante appunto una slitta o un carro rudimentale senza ruote:

Or sachiés que por ce qe en toute celle jornee ne poent aler chavalz por la glace et por la boe, car cest.XIII. jornee sunt entre .II. montagnes en

11 Si veda nel Lemmario anche la voce curata sempre da Eugenio Burgio in Simion, Burgio 2015. 
une grant valee, e por ce hi est la glace e la boe tel com je voç ai conté. Or, por ceste caisonz ensi com je voç ai dit, les chevaus ne i puet aler. E, por ce qe carette con roes ne i poroit alere, ont il fait faire une trejes qe ne a roies, $\{\mathrm{n}\}$ e sunt faites en tel mainere q'eles alent $\{$ sor $\}$ por la glace e por la boe e por le fanc, si qe ne i se fiche mie. (F CCXVI 9-10)

Tragule, però, non si oppone solo a $\mathrm{F}$, ma è isolato anche dal resto della tradizione, che presenta forme molto simili a quella del franco-italiano, da tregia in $\mathrm{Z}$ a treçe in VB, fino a traze in VA, pure antigrafo di Pipino. La scelta di introdurre il latinismo rinvia dunque a una chiara posizione da parte di Ramusio, e mostra come l'interferenza linguistica possa manifestarsi anche in presenza di un certo grado di consapevolezza da parte del traduttore. La conservazione del lemma, in altre parole, è sì dovuta a un'interferenza del modello latino, ma si tratta di un'interferenza in certa misura 'volontaria': rispetto al ben poco elegante tregia di $\mathrm{Z}$, il tragule di $\mathrm{P}$ doveva apparire molto più prossimo, nelle idee dell'umanista, al buon latino nel quale era stato redatto, a suo credere, il racconto di Marco.

\section{La parte di Ramusio. Errori di copia ed errori di traduzione}

Ultimo anello della catena testuale, il testo di Ramusio è allo stesso tempo raccoglitore finale degli esiti dell'interferenza nei testi che l'hanno preceduto e, a sua volta, generatore di casi nuovi. Se il Ramusio editore 'eredita' i prodotti del contatto linguistico dei suoi modelli con le loro rispettive fonti, il Ramusio traduttore viene a trovarsi egli stesso in una situazione propizia al prodursi dell'interferenza. Ai casi visti finora, quindi, nei quali Ramusio è un 'ripetitore' in certa misura passivo delle lezioni più o meno felici dei suoi modelli, se ne aggiungono altri, nei quali anche l'umanista sembra aver avuto parte attiva.

Un esempio si legge nel capitolo 34 del libro I, dedicato alla provincia di Qarqan. La pericope 4 informa che nell'antica città-oasi, snodo importante nella Via della seta

Vi sono molti fiumi grossi, nelli quali si trovano molti diaspri et calcedonii che si portano fino ad Ouchach a vendere, et di quelli ne fanno gran mercantia, per esservene gran copia. (R I 34 4) (corsivi aggiunti)

Il toponimo Ouchach non trova conferme nel resto della tradizione manoscritta. Infatti, se il toledano è lacunoso, la redazione di Pipino permette di riconoscere, dietro alla forma Ouchach, semplicemente la regione del Catai: "copiose preciosi lapides, iaspides et calcedonii magni valoris qui a negociatoribus deferuntur ad provinciam Cathay» (P I 43 2). È sempre F, però, a offrire l'eziologia dell'errore di Ramusio: 
Il hi a fluns qe moinent diaspes et calcedon, les qualz portent a vendre au Cata et n'ont grant profit car il en ont aseç et bones. (F LV 5)

Com'è facile comprendere, l'origine dell'errore risiede nella variante corrotta del toponimo Catai, che - unita alla precedente preposizione $a u$ - generò aucata e poi ouchach. La validità della ricostruzione è comprovata dalla rubrica che Ramusio antepone al capitolo, dove il toponimo di F è ancora più riconoscibile e presenta la forma Aucata:

Della provincia di Ciarcian, et delle pietre de diaspri et calcedonii che si trovano nei fiumi et sono portati in Aucata; et come gli habitanti fuggono nei diserti come passa l'essercito de' Tartari. (R I 34 0)

La presenza della lezione 'oscura' Ouchach in corrispondenza di una lezione pienamente intelligibile in Pipino, dimostra che in questo punto la fonte di Ramusio doveva essere il codice Ghisi, e che questo - così come la versione $\mathrm{Z}$ di cui era relatore - recava anch'esso come F una variante corrotta del toponimo.

Il caso si presta a qualche osservazione sulla dialettica tra errore di copia ed errore di traduzione, dialettica particolarmente complessa in un caso di trasmissione indiretta a vari livelli, dove più operazioni di traduzione di succedono. Nella lezione Aucata/Ouchach, infatti, è possibile distinguere due errori successivi. Il primo è la scrizione univerbata della preposizione au/ou con il termine successivo; il secondo consiste nella scorretta analisi della stringa aucata/ouchach, interpretata come un toponimo e fatta precedere dalla preposizione di luogo in/ad. La scrizione continua aucata è evidentemente un errore di copia imputabile al copista distratto o affaticato; allo stesso modo, è ovvio errore di copia la produzione della forma più eccentrica ma equivalente di ouchach. L'interpretazione delle due grafie come toponimi, e l'aggiunta della preposizione di luogo, invece, è operazione qualificabile come un errore di traduzione: è il traduttore a non riconoscere la preposizione francese au/ ou e a creare Aucata/Ouchacha. Quanto all'altezza alla quale questi errori rimontano, se la forma au Cata deve risalire già al modello franco-italiano di $Z$, si può dubitare sulla forma ouchacha, che può essere l'esito di più errori di copia sovrapposti, dall'agglutinazione della preposizione al passaggio $t>c$. Più complicato è, invece, definire il livello dell'errore di traduzione, ed è lecito chiedersi se la mancata analisi della preposizione francese e l'aggiunta di una nuova preposizione risalga a Ramusio o già al suo modello.

Che si collochi a livello di R o del suo antigrafo, tuttavia, la forma del presunto toponimo Aucata/Ouchach è indicativa del particolare trattamento che, esattamente come i realia esotici, subiscono i nomi propri (NP) in traduzione. Caratterizzati da un rapporto biunivoco ed esclusivo con la realtà extralinguistica, i NP appaiono come segni che non solo rappresentano ma coincidono con il loro designatum, che non può dunque essere identificato se non con l'etichetta che gli è propria. Questa specificità del NP gli confe- 
risce, come per gli appellativi indigeni, una certa resistenza al processo di traduzione, offrendo indizi preziosi per ricostruire la genealogia del testo. Il caso di Aucata/Ouchach, infatti, mostra come davanti a un NP il traduttore 'sospenda' la sua attività di trasposizione linguistica e si trasformi in un semplice copista, accogliendo il termine tal quale. Questa sospensione dell'attività traduttoria, inoltre, ha effetti anche sulla capacità di analizzare correttamente il sintagma, specialmente se presente in forma corrotta nel modello. Considerata parte di un NP, quindi, la preposizione au/ou non è stata riconosciuta, e si è così conservata lungo la catena testuale offrendo una riprova decisamente solida della derivazione francese di $\mathrm{Z}$.

Ora, esiste una terza variante del toponimo che consente di presentare un altro errore di traduzione, di natura più concettuale e più facilmente ascrivibile al Ramusio. Dopo una lunga e dettagliata esposizione sui costumi militari dei Mongoli, il capitolo I 47 si conclude in questo modo:

Tutto quello che vi habbiam narrato è nella vita et costumi de' rettori de' Tartari. Ma al presente sono molto bastardati, perché quelli che conversano in Ouchacha osservano la vita et costumi di quelli che adorano gl'idoli et hanno lasciata la sua legge; quelli che conversano in Oriente osservano i costumi d'i Saraceni. (R I 47 13-4)

Il testo introduce una distinzione tra i Tartari che vivono in Oriente, con abitudini analoghe ai musulmani, e i Tartari che vivono «in Ouchacha». Ancora una volta, il toponimo è l'esito di un'interferenza dovuta a una forma oscura nel modello franco-italiano di partenza. La versione F presenta, infatti, una lezione allo stesso tempo più semplice ma istruttiva sulla genesi del misterioso toponimo in $\mathrm{R}$ :

je vos di que orendroit sunt mout enbatardi, car celz que usent au Cata<i> [ms. aucata] se mantienent a lles $\mathrm{u}<\mathrm{sa}>\mathrm{jes}$ [ms. uies] et a la mainere et as costumes des ydres et ont laisé lor loy, et celz que usent en Levant se tienent a la mainere de saraçin». (F LXIX 24)

Come si vede, in F l'opposizione è tra Tartari «que usent en Levant» e Tartari «que usent $a u$ Cata<i>». L'integrazione dell'ultima vocale chiarisce la grafia 'sbadata' aucata, ed è confermata da L 60 24:

Hec dicta de moribus Tartarorum vera sunt de veris Tartaris: quia qui ad Catay conversantur ydolatrarum moribus maxime sunt affecti fidemque suam in plurimum relinquerunt, et qui in Orientis partibus conversantur ad Sarracenorum consuetudines sunt reducti.

Di fronte a un'altra lacuna nello Z toledano, la lezione Ouchacha e il suo legame con l'errore di copia di F consentono ancora una volta di riconoscere 
lo Z Ghisi come modello di Ramusio per il passo in questione, ipotizzando la resistenza di una forma corrotta aucata dal modello francoitaliano di Z all'archetipo della versione fino al codice Ghisi.

La forma Ouchacha, però, non è isolata nella versione ramusiana, e rende questo caso più complesso del precedente. Il toponimo, infatti, richiama alla memoria il capitolo d'esordio dei Viaggi, che racconta come i fratelli Polo, facendo ritorno a Costantinopoli dopo la visita a Berke, «vennero ad una città detta Ouchacha, qual è nel fin del regno di questo signor de' Tartari di Ponente» (R I 1 7). In questo caso, Ouchacha è lezione corretta corrispondente a Oucaca/Ouchacea in F 2 (11-2) - «s'en alent a une cité qui avoit a nom Ouchacea, qui estoit la fin dou reingne dou sire dou Ponent; et da Oucaca si partirent et pasent le flum de Tigri [...]»-e identificata con Ügek, città dell'Orda d'Oro sulla sponda destra del Volga. Il passaggio aucata > in Ouchacha, quindi, può essere interpretato non solo come l'esito di una mancata analisi della preposizione ou, ma anche di una possibile confusione - attribuibile al Ramusio - tra il toponimo derivante dall'agglutinazione e quello precedentemente citato all'altezza dell'inizio del suo testo. ${ }^{12}$

\section{$6 \quad$ Fautes de sens}

I casi analizzati finora mostrano come si possano distinguere due livelli di azione dell'interferenza linguistica sul piano lessicale. Il primo livello è prettamente linguistico e consiste nella produzione di lemmi formalmente devianti che, all'interno della lingua d'arrivo, manifestano caratteristiche grafico-fonetiche o morfematiche della lingua di partenza: ne sono esempi il prestito non integrato vernique, il latinismo tragule e le preposizioni francesi agglutinate au/ou. Il secondo e più profondo livello del contatto linguistico, invece, produce alterazioni nel senso di quanto raccontato, generando delle lezioni interpretabili come errori semantici: il finto ermellino rondes, il toponimo inesistente Aucata/Ouchach e la confusione Ouchacha/Ügek.

L'origine degli errori semantici rinvia necessariamente a un'operazione fondamentale di qualsiasi processo di traduzione, ovvero un'operazione di comprensione. Comprendere un testo, infatti, implica non soltanto un'attività prettamente linguistica - l'analisi e la decodificazione delle strutture della lingua del testo di partenza - ma anche un'attività di tipo più squisitamente cognitivo. In questa seconda operazione, il lettore mette in rapporto le informazioni ricavate o inferite dal testo con le proprie conoscenze

12 Per il complesso caso Aucata/Ouchach/Ouchacha, si vedano le voci Aucata e Ouchacha curate da Eugenio Burgio nel «Lemmario» di Simion, Burgio 2015. 
pregresse, tratte o da passaggi anteriori dello stesso testo o dalla propria esperienza extralinguistica. Scrive Jean Dancette $(1989,94)$ :

Ainsi, la compréhension d'un texte suppose [...] la mise en œuvre de plusieurs mécanismes successifs, dont la reconnaissance des schémas, scénarios, etc. invoqués par le texte et le remplissage des informations manquantes mais contenues dans la structure de connaissances. La compréhension consiste alors à saisir les relations temporelles, [...] à appliquer des connaissances antérieures que l'on a sur des personnages [...] et sur des objets [...], à inférer correctement des faits non exprimés.

Concepita come un'attività duplice, la comprensione presenta occasioni di errore sia a livello di decodifica linguistica sia di meccanismi cognitivi. Un'errata analisi linguistica, per esempio, è alla base dell'agglutinazione della preposizione au/ou nei toponimi Aucata/Ouchacha e della corruzione roi des > rondes. L'assimilazione di Aucata/Ouchaca a Ügek, e l'invenzione di un animale cui attribuire il problematico appellativo rondes sono però risultati di operazioni cognitive, cattive inferenze o sovra-interpretazioni. Lungi dal costituire due operazioni a sé stanti, codifica linguistica e interpretazione sono quindi fortemente interconnesse da un rapporto di complementarietà e compensazione:

Avec de faux présupposés on peut faire des entorses à la syntaxe, au lexique, à la sémantique; ou, au contraire, malgré de faux présupposés, le décodage linguistique peut permettre de construire un sens exact et, en cours de route, de modifier la première hypothèse. (Dancette 1989, 95)

Un altro esempio di errore semantico, dove al contatto linguistico si aggiunge una difficoltà cognitiva, riguarda un caso a metà via tra la coppia vernique/verniqua e il fantasioso rondes. Nel capitolo III 8, relativo alle isole indonesiane di Sondur e Condur, la versione R legge: «Et vi nasce una sorte di frutti chiamati berci, che sono domestici et grandi come limoni, et molto buoni da mangiare». Come rondes, anche berci non ha alcun corrispettivo reale, ed è il frutto - fuor di metafora - dell'accoglimento di una lezione del testo di P III 114 (il solo a parlare di limoni): «In hac provincia crescunt birci qui domestici sunt et magni ut limones, qui valde boni sunt». ${ }^{13}$

La lezione «grandi come limoni» rappresenta un altro caso di trasmissione verticale di un errore complesso e 'stratificato', che consente un

13 Queste le pericopi corrispondenti nelle altre versioni 'fonti' di R: F 163 5: «En ceste provence naist le ber $r$ >çi domesce en grandisime quantité»; Z 97 6: «In ista provincia nascitur berci domesticum in multa quantitate»; L 148 3: «Hic nascitur verçi domesticum in maxima quantitate»; VB 132 5: In questa provincia nase verçi assai et ecian muschio e habano in gran quantitade. 
nuovo breve affondo sulla dialettica copia/traduzione. Alla base dei limones di Pipino, infatti, sta la corruzione di una lezione mutuata da VA CXXV 4: «In questa chontrà nasie birzi domestegi che èno chome lovini, et sono molti boni». La genesi di lovini - spiega Rinaldin (in corso di stampa) nella scheda corrispondente del glossario - è un errore di VA che risale verosimilmente a una lezione analoga a quella che presenta la versione $\mathrm{F}$ parlando della regione di Coilum (Quilon, oggi Kollam):

Coilum est une roiames qe l'en trouve ver garbi quant l'en se part de Mabar et l'en ala .Vc. miles. [...] Et or vos vuoel contere qe se treuve en ceste roiame e qe hi naist. Or sachiés q'il hi naist le berçi coilomin, que mout est buen. (F CLXXIX)

L'aggettivo di provenienza coilomin è dunque all'origine, in VA, del complemento di paragone «chome lovini». L'errore sta a metà strada tra un errore di copia e un errore di traduzione, confermando la complementarietà delle due operazioni. Sicuramente, infatti, il traduttore non ha compreso il senso dell'aggettivo di provenienza coilomin e ha tradotto in modo errato; tuttavia, la lezione «chome lovini» si spiega anche come il risultato di una cattiva lettura, dove chome può derivare dall'interpretazione di coi come com, e lovini da una diversa analisi paleografica della sequenza di tratti min > uini. La difficoltà di scindere tra errore di copia ed errore di traduzione è però più evidente nella lezione di Pipino, ugualmente analizzabile come il risultato di un'operazione di traduzione o di un'operazione di copia. Lemma fortemente connotato in senso regionale, lovini è attestato - secondo il Corpus OVI - solo al plurale e in due soli testi: il libro padovano di Agregà de Serapiom (1390, Ineichen 1966) e le Rime di Antonio da Ferrara (XIVs, Manetti 2000). È dunque possibile ipotizzare una difficoltà di comprensione da parte del traduttore bolognese Pipino, che potrebbe aver banalizzato in limoni. Tuttavia, la lezione limoni può anche essere spiegata restando sul solo piano paleografico e congetturando nuovamente una diversa interpretazione dei tratti uini > imi, seguita da un'inversione sillabica *lomini > limoni. Il rapporto copia/traduzione, insomma, resta delicato e si rivela spesso difficile distinguere tra errata traduzione o errata trascrizione. Inoltre, traduzione e copia coincidono all'atto pratico e rappresentano due volets di un'operazione doppia, intellettuale e meccanica insieme; in questo senso, il traduttore stesso non è che un tipo particolare di copista, anche lui soggetto ai rischi di errore che la trascrizione comporta.

Ma torniamo a berci. Alla base della lezione «una sorte di frutti chiamati berci» possiamo collocare due meccanismi. In primo luogo, essa deriva da un meccanismo d'inerzia rispetto a un presunto frutto esotico il cui appellativo è, come consueto, pigramente trasferito come prestito e anticipato dalla solita stringa introduttiva «chiamati...». In secondo luogo, la lezione 
è anche il risultato del mancato riconoscimento, da parte di Ramusio, di una pianta già menzionata nel paragrafo appena precedente e da lui stesso chiamata verzino: «In quest'isola nasce verzin domestico in gran quantità; oro hanno in tanta abondanza che alcuno non lo potrebbe mai credere» ( $R$ III 8 4).$^{14}$ All'errore linguistico, dunque, si aggiunge un errore cognitivo: da un lato, Ramusio traduce erroneamente birci in berci (errore linguistico), dall'altro, non si accorge che il fitonimo da lui appena creato rinvia a una pianta già incontrata e opportunatamente tradotta come verzino. ${ }^{15}$

Oltre a involontarie défaillances linguistiche o cognitive, l'errore semantico può però scaturire anche da un intervento consapevole e volontario del traduttore, che - sulla base di errate inferenze della sua personale esperienza - corregge una lezione autentica e introduce un'innovazione erronea. Ne è un esempio il passo in R III 24 , relativo al ricco palazzo del signore di Zipangu (il Giappone). Scrive Ramusio che il palazzo è

tutto coperto di piastre d'oro, secondo che noi copriamo le case o vero chiese di piombo, e tutti i sopracieli delle sale e di molte camere sono di tavolette di puro oro molto grosse.

Il termine sopracieli è invenzione di Ramusio, ed è sostituzione antonimica rispetto alla lezione condivisa da $\mathrm{F}$ e da tutte le sue fonti ( $\mathrm{Z}, \mathrm{P}, \mathrm{VB})$, ovvero pavimant/pavimenta/pavimentum/pavimento:

\begin{tabular}{llll}
\hline F CLVIII 8 & Z 92 91 & P III 2 & VB 127 5 \\
\hline vos di qe tout le & Etiam omnia & pavimentum aularum & Et ancho el \\
pavimant de seç & pavimenta & atque, camerarum & pavimento del dito \\
cambres, qe aseç hi & camerarum, que & multarum aureis & palasio è coperto del \\
ni a, sunt ausint d'or & multa sunti bi, & tabulis est & dito oro, e questo è \\
fin bine gros plus de & sunt de auro, valde & opertum... & $\begin{array}{l}\text { nella sala et ancho } \\
\text { in alchune delle } \\
\text { II doies... }\end{array}$ \\
& grossa... & & chamere... \\
\hline
\end{tabular}

Come spiega Burgio (2011, XLVI-XLVII), l'alterazione consapevole del testo è analizzabile come un conflitto tra l'enciclopedia mentale di Ramusio e il testo dei suoi modelli, tale per cui il richiamo all'esperienza dei tetti ricoperti di piombo delle chiese gli fa ritenere più verosimile la copertura dorata di un soffitto piuttosto che di un pavimento.

14 Ramusio traduce Z 97 6-9: «In ista provincia nascitur berci domesticum in multa quantitate». Il passo corrisponde a F CLXIII 5: «En ceste provence naist le be〈r»çi domesce en grandisime quantité».

15 Per berci/verzino cf. Simion, Burgio 2015 («Lemmario», s.v. «Berci» a cura di Eugenio Burgio). Ringrazio Anna Rinaldin per aver condiviso la scheda «Verzino» allestita per il «Glossario» per l'edizione ramusiana di Simion, Burgio 2015 (Rinaldin, in corso di stampa). 


\section{Meccanismi cognitivi e poligenesi}

La caratteristica dei meccanismi cognitivi alla base di errori come quello commentato in precedenza è di essere universali. In altre parole, se i processi di decodificazione linguistica possono produrre errori la cui natura rivela quali lingue ne sono alla base, la componente interpretativa dell'errore segue generalmente modalità costanti e indipendenti dal tipo di lingue coinvolte. Così, alcuni processi cognitivi si ritrovano anche in versioni genealogicamente lontane e irrelate, risultando trascurabili sul piano ecdotico, ma significativi da un punto di vista culturale.

Alcuni esempi riguardano, ancora una volta, lo statuto eccentrico dei nomi propri. Nel capitolo II 31, la redazione R si sofferma sul castello di Thaigin, costruito per volere di «un re chiamato Dor». L'antroponimo è la corruzione dell'originario roi d'Or di F CVII 2: «un biaus castel, qui est apellés Caiciu, le quel fist faire jadis un rois qe fu apellés le roi d'Or». L'errata analisi del NP è comune anche a V, che scrive che il castello fu edificato da «uno re chiamato Dor» (V 51 7). Il caso è da manuale, e incoraggerebbe spiegazioni frettolose: preceduto dalla sequenza «qe fu apellés», il NP d'Or è stato analizzato scorrettamente da V, che dunque potrebbe sembrare la fonte di Ramusio per questa pericope. Tuttavia, se si estende lo sguardo agli altri testimoni, ci si accorge che un travisamento analogo è presente in VB - che parla di un «re Doro nobellissimo segnore nel suo tenpo» (LXXVI 1) - e in P, che normalizza il NP in Darius: « castrum pulcherrimum Caycuy, quod edificavit rex quidam Darius nomine» (II 30 1).

Un altro caso di meccanismo cognitivo etero-genetico che non coinvolge, tuttavia, la redazione di Ramusio è il seguente. Nel capitolo XXII, la redazione F ci informa che in Georgia regna un re chiamato Davit Melic, ovvero 'Davide re': «En Jorgienie a un roi qi est apelés par tout tens Davit Melic, que vent a dir en fransois Davit roi» (F XXII 2). Il passo si legge identico in Z 41 - «In Iorgia est quidam rex qui David Melic totis temporibus nuncupatur, quod in lingua Galica dicitur Rex David - e così lo traduce Ramusio: «In Zorzania è un re che in ogni tempo si chiama David Melich, che in lingua nostra si dice re David» (R I 5). La lezione di Ramusio è corretta e, anzi - sostituendo «in lingua Galica» con «in lingua nostra» - rimedia alla défaillance di $\mathrm{Z}$ che, copiando dal proprio modello franco-italiano, presenta «Rex David» come una traduzione francese. Il punto, però, è un altro. Correttamente analizzato da Ramusio, David Melic è composto da un nome proprio (occidentale) e da un nome comune che funge da apposizione, e che indica una carica istituzionale asiatica comparabile a quella di un sovrano e traducibile con 're'. ${ }^{16}$

16 Come spiega la scheda di Vito Santoliquido in Simion, Burgio 2015 («Lemmario»), Melich corrisponde all'arabo malik (pl. mulūk) e sta primariamente per 're, monarca'; il lemma 
Il sintagma, però, ha dato filo da torcere a più di un copista/traduttore. Restando tra le fonti di Ramusio, la versione di Pipino omette il nome e il titolo del personaggio, limitandosi ad affermare che «Çorçanie provincia regem habet Tartarorum regi tributarium» (P I 14 1). Il silenzio del domenicano deriva verosimilmente dall'imbarazzo nei confronti di una lezione eccentrica del suo modello. VA XIII 1, infatti, incorpora il NP Davit entro l'arabismo Melic e ne deriva un unico ed eccentrico Mandemilich: «In Zorzania è uno re ch'è apellato senpre Mandemilich, che è a dire in nostra lengua Davit re, ed è sotoposto al Tartaro». Il caso più interessante, però, riguarda la versione V. Tratta, come dimostra Simion (2011), da un modello latino, V 12 1-2 legge: «<I>n Zorzania in quel tempo era uno re chiamado Davit Mioliorotis, che in lengua galilea vien a dir 'Davit Re'». Oltre al travisamento galica > galilea, V trasforma Davit Melic in Davit Mioliorotis: dietro quest'ultimo termine apparentemente così fantasioso si cela in realtà l'agglutinazione entro il lemma esotico del pronome latino totis (cf. Z 4 1: «rex qui David Melic totis temporibus nuncupatur»), secondo i passaggi David Melic totis > Davit Meliototis > Davit Mioliorotis (Simion 2011, 33-4). Come si vede, la nuova creazione lessicale consente, in modo analogo a casi sopra citati, di definire quale sistema linguistico ha prodotto l'interferenza, dimostrando che la fonte di V doveva essere redatta in latino.

Ora, il termine melic si ripresenta anche altrove nel racconto di Polo, e le varie redazioni manoscritte ne recano occorrenze sparse senza una sistematica corrispondenza con il resto della tradizione. Eppure, molte di queste lezioni sono caratterizzate da un fenomeno identico, ovvero la trasformazione del nome comune melic in nome proprio di persona. Nel capitolo dedicato alla città di Calaiati, sulle coste dell'Oman, Ramusio scrive che essa «è sottoposta al melich di Ormus» (R III 43 2), e aggiunge successivamente:

E molte volte che 'l melich di questa città, qual ha patti et obligatione col re di Chermain et li è suddito, non lo vuol obedire, perché 'l detto l'impone qualche datio oltra l'ordinario et esso ricusa di pagarlo, subito il re li manda un essercito per constringerli per forza; lui si parte di Ormus et viene a questa città di Calaiati, dove stando non lassa entrare né passare alcuna nave: dal che advien che 'l re di Chermain perde i suoi dretti e, ricevendo gran danno, è necessitato a far patto con il detto melich. (R, III 43 7)

deriva dall'antica radice semitica $m-l-k$, la quale racchiude il significato di 'proprietà' e, per estensione, 'dominio' e 'governo'. 
La lezione corrisponde a F CXCVI 7, dove si legge analogamente:

Et mantes foies en a le melic de ceste cité grant pat dou soudan de Creman, cui il est sontpost: car, quand cel soudan met aucu<n〉 dasio au melic de Curmos, ou aucun autre de sez frers, et cesti ne le velent doner, e le soudan hi tramest host por elz esforcer, il se partent de Curmos et entrent es nes e s'en vienent a ceste cité de Calatu et iluec demorent et ne laissent passer nulle nes, dont le soldan de Cremain en a trop grant domajes, e por ce convient que il face pes au melic dou Curmos e ne li tolt pas tant monoie com il li demandoit.

\section{Ecco invece cosa reca V 112 5:}

E molte volte Milia, signor de questa zitade, àno gran pati chon el Soldan, el qualle ello hè sudito, perché quando el Soldan mete algun dazio a Milia, over ad alguni dî fradelli, e questi non volesse, el Soldan manda el suo' exerzito per chazar quelli per forza; elli se parteno e monta suxo le nave, donde el Soldan ne rezeve gran dano, per tal ch'el è de bexogno ch'el faza paxe chon questo Milia. Et sì non àno tanta quantità de pechunia chomo lo i aveva domandada questo Milia.

Come si vede, $\mathrm{V}$ interpreta l'appellativo ignoto melic come il nome proprio del sovrano. Quel che importa qui, però, è che quest'errore cognitivo non è proprio di V, e si trova anche in testimoni da esso stemmaticamente lontani. Ecco, ad esempio, come appare questo stesso passaggio nel testimone catalano (Kc) Firenze Ricc. ms. 2048, uno dei tre relatori della redazione $\mathrm{K}$, epitome catalana della seconda metà del Trecento: ${ }^{17}$

〈C〉alatu és gran ciutat e és luny de Dufar bé DC miles vés mestre, e és a la mar. [...] Aquest seyor, qui s'apella Melic, és hom del saudà de Caramon. E can lo dit saudà pren guerra $a b$ lo dit Melich, el se·n vén an esta ciutat e no lexa passar lo naveli, per què lo saldà pert sa renda e cové-li que per forsa fassa pau ab lo dit $M e l<i>c h$, él se·n vén an esta ciutat. Atressí ha lo dit Melic an aquest golf IIII castels qui són gran gardia e defencion del golf. (Kc 101 1-5)

La lezione «Aquest seyor, qui s'apella Melic», dove la maiuscola è aggiunta dall'editore (manca un criterio regolare maiuscola/minuscola nel manoscritto), lascia spazio a un'interpretazione analoga a quella di V. Pur non deformando il nome comune melic, il testo catalano sembra intenderlo

17 Le tre citazioni seguenti, tratte dalla redazione $\mathrm{K}$, rinviano alle edizioni in Reginato 2015-6. 
come il nome proprio del seyor della città. Lo stesso nel testimone francese (Kf) della stessa famiglia, il ms. Città del Vaticano, BAV, Ott. Lat. 2207:

Calatu est une grant cité et est moult loing de Dufar bien $\mathrm{v}^{\mathrm{c}}$ milles vers mestre, et si est sur la mer. [...] Et ce seigneur qui s'appelle Mellich est homme du soudant de Germon. Et quant ledit soudant print guerre audit Mellich, il s'en vient en ycelle cité et ne lesse passer nulles nefs ne nulz navires, par quoy le soudant pert sa rente et convient que par force il face pais audit Mellich. Et en ce goulf a IIII chasteaux qui sont grant garde et deffendement du goulf. (Kf 100 1-5) ${ }^{\mathbf{1 8}}$

Meno dubbi lascia il terzo testimone del gruppo, l'aragonese Madrid El Escorial Z.I.2 (Ka), che elimina l'articolo dalla lezione «fassa pau ab lo dit Mel<i>c$\mathrm{h} » / \ll$ face pais audit Mellich» e usa la sola preposizione con seguita direttamente da Mellich, interpretandolo quindi senza dubbio come nome proprio:

Palatu es grant ciudat et es luent de Dusfar bien DC millas devés maestro, la qual es muy rica et es riba mar. Las gentes son moros. [...] Et senyor d'esta ciudat se clama Mellich, et es hombre del soldan de Thermon. Et quando el dicho soldan toma grant guerra con Mellich, él s'ende viene en aquesta ciudat et non dexa passar nengun navilio, por la qual razón el soldan pierde grant partida de sus rendas assí que le conviene fer paz con el dicho Mellich. (Ka 67 2-6)

Melic, infine, appare anche in un passo della redazione toscana TA, dove indica un vicario del sultano di Hormuz incaricato di sorvegliare il prigioniero Argon; anche la versione toscana del Trecento trasforma il nome comune nel nome proprio Melichi/Milichi:

Lo soldano si era uomo molto lusurioso, sí che si pensò di ritornare a la terra e di pigliare molte belle donne che v'erano. Alora si partío, e lasciò uno suo vicario ne l'oste, ch'avea nome Melichi, che dovesse guardare bene Argo; e così se n'ando a la terra, e Milichi rimase. (TA 201 1; Bertolucci Pizzorusso 1975, 309)

Privo di corrispondenza in Ramusio, lo stesso passaggio si legge nella redazione $\mathrm{V}$, che anche in questo caso non manca di originalità:

Hor questo Achomach, el qualle era molto luxorioxo, diterminò de andar ala chorte a solazarse chon le suo' damixelle; et lassò al signor de tuto lo exerzito, el qualle nomea Melichamus, in guardia de Argon; et sì li disse

Anche per Kf e Ka, la maiuscola è aggiunta dall'editore. 
ch'el dovesse vardar Argon quanto la so vita, et ch'el dovesse vegnir ala chorte in pochi dì per salude dela so zente. Et Melichamus disse ch'elo hobedirave i suo' chomandamenti. (V 116 63-64) ${ }^{19}$

\section{Conclusioni}

In conclusione, la selezione dei casi raccolti in queste pagine ha seguito due obiettivi: da un lato, mostrare come l'indagine lessicale possa, soprattutto in tradizioni plurilingui, coadiuvare la pratica filologica e rivelarsi un valido strumento di analisi testuale; dall'altro, offrire delle osservazioni di validità generale e metodologica su alcuni aspetti dell'interferenza linguistica applicata a un testo scritto, esito di successivi passaggi di copia e traduzione.

In primo luogo, il riconoscimento di quale sistema linguistico agisce su un determinato testo si è rivelato fondamentale per identificare la lingua della sua fonte: così, il latinismo tragule tradisce l'uso della versione di Pipino, mentre la permeabilità al francese della redazione $\mathrm{Z}$ - visibile in casi come vernique - dimostra la sua derivazione franco-italiana.

In secondo luogo, l'analisi di alcuni prestiti esotici ha messo in luce da un lato come la conservazione di forme erronee sia facilitata nei casi di realia asiatici (sic?) e nomi propri (da rondes a Aucata/Ouchach), dall'altro come l'interferenza in Ramusio dipenda talvolta dal suo personale uso delle fonti (la corretta primazia del Ghisi e il penchant per Pipino), dalle sue intenzioni editoriali (la ricerca di esaustività) e dalle sue idee sull'originale poliano (che riteneva scritto in latino).

In terzo luogo, le osservazioni sulla dialettica tra errore di copia ed errore di traduzione hanno mostrato come spesso sia difficile se non impossibile precisare a quale operazione - meccanica o intellettuale - risalga la genesi dell'errore. Entro le operazioni di traduzione, poi, la distinzione tra processi di codificazione linguistica e meccanismi cognitivi ha condotto all'analisi di fautes de sens che non solo si mostrano linguisticamente devianti, ma anche semanticamente erronee: così, l'errore linguistico alla base dell'agglutinazione della preposizione in Aucata/Ouchach facilita la successiva confusione tra questa città immaginaria e la reale Ouchacha/ Ügek. A errori inconsapevoli, poi, si sono accostate innovazioni consapevoli, dove l'interferenza non è più linguistica ma culturale, come nell'inversione «polare» (Burgio 2011, XCVII) tra pavimant/sopracieli.

Infine, la riflessione sulla portata universale di alcuni meccanismi cognitivi, e sulla loro presenza in versioni distanti dal punto di vista genealogico,

19 Cf. le voci David e Melich a cura rispettivamente di E. Burgio e Valentina Santoliquido in Simion, Burgio 2015 («Lemmario»). 
ha mostrato come l'analisi lessicale richieda, come ogni analisi filologica, una particolare attenzione nel distinguere tra esito monogenetico ed esito passibile di poligenesi. 


\title{
L’ambiguità del teonimo poliano Natigay-Načigay
}

\author{
Elisabetta Ragagnin \\ (Freie Universität Berlin, Deutschland/Università Ca' Foscari Venezia, Italia)
}

\begin{abstract}
This contribution offers an in-depth discussion of the Polian theonym Natigay-Načigay, highlighting its relation with theonyms and shamanistic terms occurring in the European travel literature and/or in Turkic and Mongolic sources.
\end{abstract}

Keywords Mongolian philology. Altaic philology. Etymology. Marco Polo Studies. Shamanism.

Il Devisement dou monde offre preziose informazioni riguardo al mondo spirituale dei Ta(r)tari, ${ }^{1}$ a cui Marco Polo dedica un ricco e ampio capitolo» a un Dio alto, sublime e celeste - il cui nome, noto in altre fonti, era tenggeri $-^{2}$ il viaggiatore veneziano descrive un'altra divinità:

La legge et fede de' Tartari è tale: dicono esservi il Dio alto, sublime et celeste, al qual ogni giorno col torribolo et incenso non dimandano altro se non buon intelletto et sanità; ne hanno poi un altro che chiamano Natigay, ch'è a modo di una statua coperta di feltre o vero di altro, et ciascheduno ne tiene uno in casa sua. Fanno a questo dio la moglie et figliuoli, et pongongli la moglie dalla parte sinistra et li figliuoli avanti di lui, quali pare che li facciano riverenza. Questo dio lo chiamano dio delle cose terrene, il quale custodisce et guarda i loro figliuoli et conserva le bestie et le biade, al quale fanno grande riverenza et honore; et sempre quando mangiano toglieno della parte delle carni grasse, et con quelle ungono la bocca del dio, della moglie et de' figliuoli; dapoi gettano del brodo delle carni fuor della porta agli altri spiriti. Fatto questo, dicono che 'l loro dio con la sua famiglia ha havuto la parte sua, et poscia mangiano et bevono a lor piacere. (R I 46 1-4)

1 Sul termine 'ta(r)taro', impiegato nel Medioevo per designare i Mongoli, cf. de Rachewiltz 1971, Golden 1992, Tatár 2003, Atwood 2004, 528-30.

2 L'etimologia di tenggeri, corrispondente alla forma antico turcica teAltro carattere misteriosoyri - documentata nelle iscrizioni dell'Orkhon (VIII sec.) - è tuttora dibattuta. Per una panoramica etimologica e per una possibile origine paleo-siberiana del termine cf. Georg 2001.

3 Do di seguito il testo di F LXIX 2-6, tradizionalmente assunto come pietra di paragone della tradizione poliana: «Et sachiés que la lor loi est tiel: car il ont un lor diu que l'apelent 
Et come habbiamo detto di sopra, questi popoli sono idolatri, et per suoi dei tutti hanno una tavola posta alta nel pariete della sua camera, sopra la qual è scritto un nome che rappresenta Dio alto, celeste et sublime: et quivi ogni giorno con il thuribulo dell'incenso lo adorano in questo modo, che, levate le mani in alto, sbattono tre volte i denti, pregandolo che li dia buon intelletto et sanità, et altro non li dimandano. Dapoi, giuso in terra, hanno una statua che si chiama Natigai, qual è dio delle cose terrene che nascono sopra tutta la terra, et li fanno una moglie et figliuoli, et l'adorano nell'istesso modo, con il thuribulo et sbattendo i denti et alzando le mani, et a questo li dimandano temperie dell'aere et frutti della terra, figliuoli et simil cose. (R II 26 1-2)

Tra le varianti del teonimo Natigay/Natigai, attestato in Ramusio, si registrano le forme Nacygai (F); Nacigay (Fr); Versigay (K); Nacigay (L); Nacigai (P); Natigai (TB); Nagani (V); Nacigai (VA); Natagai (VB), e Naçagay $(\mathrm{Z})$.

Del Dio si parla anche nel capitolo dedicato alla «regione delle Tenebre»; ${ }^{5}$ i paragrafi sulla religione sono soppressi in R III 44, ma possiamo ricorrere al testo di F CCXVI 3:

Or sachiés que il font un lor dieu de feutre et l'apellent Nacigai. Et encore li font moiliere. Et cesti .II. dieu, ce est Nacigai e sa moiere, dient qe il sunt les dieu de tere e qu'il lor gardent les bestes e les bles e toutes lor bien terenes. Il les orent; e quant il vienent qe il menuient aucune bone viandes, il en ongent la boche a lor deu; et funt bien vies come de bestes.

Nacygai, et dient que celle est dieu tereine qe garde lor filz et lor bestes et lor blee. Il li font grant reverence et grant honor, car cascun en tenent en lor maison, car il font cest deu de $\mathrm{f}\{\mathrm{r}\}$ eutre et de dras et le tenent en lor maison; et encore font la moillier de cest dieu et seç filz: la moiere metent de la senestre partie et les filz devant, et le honorent asseç. Et quant vienent a mangier, il prenent de la char grasse et n'oi<n>gent la bouche a cel dieu et a sa feme et a ssez filz. Et puis prenent dou brod e l'espannent dehors la port<e〉 de sa maison. Et quant il o<n>t ce fait, il dient que lor dieu et sa masnee ont eu lor part. Aprés ce, menjuent il et boivent, car sachiés qu'il boivent la<i>t de jumente, mes si voç di qu'il l'adobent en tel mainere ke le senble vin blance et est bone a boire, et l'apellent chemins». Per un rapido schizzo della tradizione manoscritta del Devisement dou monde cf., in questo volume, gli interventi di Samuela Simion e Alvise Andreose.

4 Il capitolo è attestato soltanto in R e, parzialmente, in Z 45 10-13, che presenta però un testo abbreviato: «Habet quilibet in domo sua statuam appensam in pariete camere, que deum altum celestem representat, vel solummodo ibi nomen dei scriptum. Et sic ipsum adorant: et levant quidem manus et dentes simul ter concutientes, ipsum rogant ut eis erroget vitam longam, alacrem et iocundam. Habent etiam in terra deorsum aliam statuam que Naçagay vocatur, deus terenorum, qui se solum habet intromitere de terenis et rerum nasentium super teram. Apud istum deum eius uxor est et filii, etcetera».

5 La provence de Oscurité di F CCXVII. 
Secondo Pelliot (1959-73, 2: 791-2 nota 295) è possibile individuare una relazione etimologica fra il teonimo poliano - in particolare con la forma «natigay», da lui giudicata come la forma autentica e più vicina all'originale -, il toponimo turcico Ötüken, ${ }^{6}$ mong. Edügen 'Terra' - che seguito dall'epiteto eke 'madre' significa 'Madre Terra' -, e Itoga/Utoga 'il Dio dei Tatari' menzionato nella Historia Mongalorum da Giovanni da Pian del Carpine. Quest'ipotesi è stata successivamente ripresa da altri studiosi, tra gli altri da Tucci, Heissig $(1970,411)$ e de Rachewiltz $(2006,430-31)$, diventando l'interpretazione corrente.

In realtà essa presenta alcune difficoltà. Innanzitutto non c'è una perfetta corrispondenza paleografica tra il lemma poliano, le forme turciche e il teonimo attestato da Pian del Carpine. In particolare risulta problematica l'occorrenza, confermata dalla maggior parte della tradizione manoscritta poliana, della consonante alveolare nasale $n$-. Già Pelliot (1959-73, 2: 791) cercò di spiegare la presenza di questa consonante iniziale interpretandola come un errore di trascrizione, ma indicando allo stesso tempo il carattere non del tutto soddisfacente di questa proposta:

it was once misread with an $n$ - belonging to the preceding word [...] and that wrong form was already substituted everywhere in the archetype of all our Mss, but such a solution is too problematic to allow us to change the spelling.

L'occorrenza di tale consonante potrebbe far pensare ad una forma 'nasalizzata' del mong. etügen: molte varietà linguistiche mongoliche e turciche dell'area siberiana, come alcuni dialetti buriati ${ }^{7}$ occidentali e le lingue sayaniche della taiga (cf. Ragagnin 2011), sono caratterizzate dalla presenza di suoni nasalizzati nei rispettivi sistemi fonetici. Si potrebbe quindi ricostruire una protoforma *nitügei che rispecchierebbe la forma mongolica itügen etügen, e che, forse, era in uso presso popolazioni che vivevano

6 Il toponimo Ötüken è documentato in vari passi delle sopraccitate iscrizioni dell'Orkhon senza precise indicazioni geografiche. Nell'opera enciclopedica del XI secolo Dīvān Luyāt at-Turk (= Compendium dei dialetti turchi) compilata dal karakhanide Maḥmūd al-Kāšyarī, il termine Ötüken identifica un toponimo ubicato fra i deserti dei Tatari nelle vicinanze degli Uiguri (Dankoff, Kelly 1982, 159), localizzabile, forse, a nord della catena dei monti Khangai, ossia nella zona compresa fra i fiumi Orkhon, Selenga e Tes; cf. de Rachewiltz $(2004,1004)$, anche per una bibliografia di riferimento. Si tratta della stessa regione in cui sorgeva la capitale mongola, Karakorum.

7 Il buriate è una lingua mongolica attualmente parlata nella omonima repubblica nella Russia orientale, e nei cui territori si trova il lago Baikal. Piccole isole linguistiche buriate sono inoltre presenti nella Mongolia interna in Cina. Per un profilo della lingua buriate, cf. Skribnik 2003. 
nella detta «Regione delle Tenebre». ${ }^{8}$ Non bisogna inoltre dimenticare che nelle lingue turciche e mongoliche è comune la distorsione fonetica dei termini legati alla religione o ad altri 'tabu' (funzione apotropaica).

L'analisi di Mostaert (1957) di un frammento buddhista del 1312, quindi cronologicamente vicino al racconto poliano - in cui è documentata la forma Načigai eke, letteralmente 'Načigai madre' (mong. eke 'madre' v. sopra) - e funzionalmente corrispondente alla Etügen Eke 'Tellus Mater' della Storia Segreta dei Mongoli, ha portato molti orientalisti, filologi e linguisti (fra cui Cardona 1975, 678) a vedere proprio in questo termine l'origine della forma paleografica poliana Načigay, documentata da F e dal codice zeladiano Z. ${ }^{9}$

Secondo questa proposta le varianti poliane con il nesso - $t i$ - potrebbero facilmente essere ricondotte a corruzioni paleografiche, per scambio $c / t$ (cf. Cardona 1975, 678 e Barbieri 2004, 225). L'analisi filologica condotta da Mostaert (1957) sul frammento buddhista permette inoltre di escludere la corrispondenza fra il teonimo poliano e il toponimo turcico Ötüken.

Per quanto riguarda invece il teonimo Itoga/Utoga attestato in Giovanni da Pian del Carpine, sono necessarie alcune precisazioni preliminari. Il lemma occorre nel cap. III 10 della Historia Mongalorum, dedicato alla religione, al concetto di peccato, alle pratiche di divinazione e purificazione e ai riti funebri dei Mongoli:

Divinationibus, auguriis, aruspiciis, veneficiis, incantationibus multum intendunt, et cum a demonibus eis respondetur, credunt quod Deus ipsis loquatur; quem Deum nominant Utoga, sed Comani Kam ipsum appellant, quem mirabiliter timent et reverentur ac ei oblationes offerunt multas et primitias ciborum et potus, et secundum responsum ipsius faciunt universa. (Daffinà et al. 1989, 240-1)

8 Con ogni probabilità, la «Regione delle Tenebre» va identificata nella Yugria, la regione corrispondente alla parte settentrionale del fiume Ob. Nel XIII secolo tali regioni erano verosimilmente abitate da varie popolazioni indigene dedite alla caccia, alla pesca, e forse all'allevamento delle renne, e di ceppo uralico, turcico e mongolico. Una importante fonte di guadagno di queste popolazioni era il commercio di pelli di animali da pelliccia che da lì raggiungevano i mercati delle importanti città sul mar Caspio e sul mar Nero. L'identificazione non è però del tutto sicura: la descrizione geografica e antropologica di Marco Polo si adatta infatti anche alle terre poste più a Oriente.

9 Nell'antica religione dei Turchi e dei Mongoli al Dio uranico tejri (turcico) e tenggeri (mongolico) si affianca la Dea tellurica - turcico yer-sub, letteralmente 'Terracqueo' (da tur. yer 'terra, suolo' e sub 'acqua') e medio mongolico Etügen Eke. Per le frequenti espressioni formulari del binomio Cielo-Terra cf. Barbieri (2004, 226). Accanto al termine yer-sub le fonti antico-turciche documentano la forma umay 'placenta', in uso anche per designare la dea protettrice delle donne e della prole, probabilmente in virtù delle qualità magiche della placenta (Clauson 1972, 164-5); cf. anche Roux de Rochelle 1990, 156-62. Sulla Madre Terra cf. inoltre Harva 1938, 243-9 e Lot-Falck 1956, 168-72. La divinità yer-sub è ancora venerata fra le popolazioni turciche altai nella Siberia meridionale, e sembrerebbe che questo culto sia di origine iranica (Harva 1938, 245). 
Come già indicato da Daffinà $(1989,416)$, l'identità fra Itoga/Utoga e il $\mathrm{kam}$ dei Comani dipende da un'errata informazione del frate francescano: in cumano infatti il termine qam è usato in riferimento allo sciamano, mentre il lemma che designa Dio è tejri/tegeri (Grønbech 1942, 241, 191). Il termine qam è attestato in turcico antico: qam 'sorcerer, soothsayer, magician'10 (Clauson 1972, 625) e anche in ciagataico: kam (قام) (mongol) 'sorcier; médicin, expérimente' (Zenker 1866-76, 684). Esistono inoltre vestigie in alcune lingue turche moderne, eg. tuvino e khakass xam, con lo stesso significato.

Sul piano etimologico Itoga/Utoga ${ }^{11}$ sembrerebbe originare da turcico ïdu $q$ - aggettivo deverbale da ï - 'mandare, spedire' il cui significato letterale è 'mandato/spedito', ma che già in turcico antico significa 'dedicato a Dio' e 'sacro' (Clauson 1972, 46). L'aggettivo ïduq significa sia 'sacro' che 'cosa sacra'. Alla possibile domanda del francescano «Che cos'è questo?», la risposta potrebbe essere stata semplicemente ïduq, cioè 'cosa sacra', da cui Itoga 'Dio'.

Un ulteriore rischio di fraintendimento è anche causato dalla facile confusione con il termine mongolo udayan 'donna sciamana' (Daffinà 1989, 416), che va probabilmente ricondotto alla stessa radice etimologica della forma turcica appena ricordata (Janhunen 1986, 101). ${ }^{12}$

Una volta stabilito che la forma «Nacigay» corrisponde senza difficoltà al mongolo «Načigay», mentre le varianti del tipo «Natigai», attestate nella tradizione, vanno ricondotte all'attività dei copisti, resta da risolvere la questione legata al sesso della divinità: nel Devisement dou monde infatti Nacigai/Natigai è una divinità maschile, dotata di consorte e prole, ma nella religione mongola Načigai è indubbiamente una figura femminile (cf. anche Barbieri 2004, 226-7).

L'aporia è stata messa in evidenza da molti commentatori; già Yule (Yule, Cordier 1903, 258) tentava di appianare i dati contraddittori spiegando il poliano «Natigai» in base alle forme Nougait o Nogat o Ongotui, documentate nelle relazioni odeporiche del XIX secolo all'interno di alcune delle lingue parlate nelle regioni siberiane limitrofe ai Mongoli quali il

10 Per una descrizione delle funzioni sociali e rituali dello sciamano, intermediario fra il mondo degli spiriti e quello degli uomini, rimandiamo a Harva $(1938,449-561)$ e Eliade (2004, 181-258).

11 Nella Hystoria Tartarorum, come già sottolineato da Daffinà $(1989,416)$, è attestata per 'Dio' la forma paleografica Codar che ha sicuramente origine dalla forma persiana khoda 'Dio'. Nelle lingue turche siberiane Altai e Khakass ci sono tuttora tracce di questo termine: Altai kuday 'Dio' (Verbickij 2005, 150a) e Khakass xuday 'id.' (Baskakov 1953, 290b). Si osservi inoltre che Mandevil'skii - citato in Banzarov $(1891,17)$ e poi in Lot-Falck (1956, 162) - riporta un'ulteriore forma paleografica per Itoga/Utoga: Iroga.

12 Anche il termine mongolico böge 'sciamano' è di origine turcica: $\leftarrow$ tur. bögü 'sage, wizard'; cf. Choi 1992. 
tunguso (il cui territorio attualmente si estende dall'attuale Buriazia alla repubblica yakuta). Tali forme chiaramente si riconducono al mongolo ongyon, oggetto di culto che ricopre particolare importanza nella cornice religioso-spirituale dell'Alta Asia. Con il termine ongyon $^{13}$ si fa riferimento allo spirito protettore della famiglia/clan nella cui abitazione esso si trova. Essi sono concretamente rappresentati attraverso immagini sacre e simulacri, fabbricati solitamente con panno di feltro, ma anche di seta, legno o bronzo. Essi presentano talvolta una forma antropomorfa, motivo per il quale alcuni viaggiatori li hanno paragonati a pupazzi e/o bambole, e 'ospitano' spiriti di vario genere: lo spirito degli antenati, di importanti sciamani deceduti, ma anche cacciatori, pescatori ed eroi che hanno protetto, in vita, la famiglia o il clan dagli attacchi di spiriti ostili. Ongyon possono inoltre essere diversi animali, gli spiriti dell'acqua e della terra nonché i corpi celesti (Harva 1938, 373; Manžigeev 1978, 62-3); ${ }^{14}$ così come divinità o spiriti venerati, pronunciare il cui nome però è tabu. Brevi informazioni sugli ongyon sono anche riportate nell'opera Jāmic at-tawārikh (= La raccolta delle storie) del ministro e poligrafo ilkhanide Rašìd-ud Dīn. ${ }^{15}$

Lo ongyon è quindi l'immagine terrena dello spirito, e come tale è oggetto da venerare.

La presenza e importanza di tali simulacri è documentata estensivamente anche dai viaggiatori francescani. La descrizione più articolata si legge nella Historia Mongalorum (III 2-3) di Giovanni da Pian del Carpine:

13 Cf. la definizione di Lessing (1995, 614a) per il mongolo classico: ongyun (pl. ongyud) 'shamanist spirit inhabiting a material object, genie, guardian spirit, tutelary deity, spirit of a deceased person, ghost; pure, holy, sacred, consacrated; tomb of a saint or eminet person, family tomb; shamanist ancestral idol; naturally white hair' e Kowalewski (1844, 353) ojgun 'pur, saint; Ongon, génie tutélaire d'un endroit; tertre sépulcral, cimetière d'une famille régnante; mets offerts aux manes'; Calmucco ojgon 'heilig, göttlich' (Ramstedt 1935, 287a), buriate ongon 'izobraženie kokogo-l. božestva, dux genij (u šamanistov), idol' (Čeremisov 1973, 356), mongolo Ordos oygon 'saint; endroit de sépulture d'un personnage éminent où se pratique un culte public; sacré, consacré à une divinité, à l'obo' (Mostaert 2009, 514b), daguro ongore 'the shaman's spirit, a departed ancestor' (Martin 1961, 202), e Yakuto oyonńor (? $\leftarrow$ mong.) 'Alter, Greis' (Stachowski 1993, 189).

14 Harva $(1938,376)$ descrivendo gli ongod buriati precisa che nella yurta erano presenti degli speciali ongod che in qualità di spiriti protettivi aiutavano le donne nel parto, e proteggevano la prole e il bestiame. Inoltre i Buriati avevano degli ongod anche all'esterno della yurta. Per gli spiriti protettori del bestiame costruivano infatti piccole casette, dei rifugi, dove essi si potevano riparare in caso di cattivo tempo. Veneravano anche certi monti, dato che secondo loro degli sciamani erano sepolti lì ed esercitavano il loro potere in quella zona circoscritta (foto degli ongyod sono riportate in Harva 1938 alle pp. 373-7).

15 «To each of the twenty four he assigned an animal to be their totem (ongqun)»; e, con riferimento al Clan Suqai'ut, un sottoclan dei Ba'arin: «they say that Genghis Khan turned loose a man off the Ba'arin clan as ongqun, just as they make horses and other animals ongqun, meaning that no one should appropriate it and that it is free and tarkhan [immune]» (si cita dall'edizione Thackstone 1998-9, 1: 105-6). 
Nichilominus habent idola quedam de filtro ad imaginis hominis facta, et illa ponunt ex utraque parte ostii stationis, et subtus illa ponunt quiddam de filtro in modo uberum factum, et illa credunt esse pecorum custodes, ac eis beneficium lactis et pullorum prestare. Alia vero faciunt de pannis sericis et illa multum honorant. Quidam ponunt illa in pulchro curru tecto ante ostium stationis, et quicumque in illo curru aliquid furatur, sine ulla miseratione occiditur. Sed quando volunt illa idola facere, omnes maiores domine conveniunt, que sunt in stationibus illis, et cum reverentia faciunt illa; et cum fecerint interficiunt ovem et illam manducant, et ossa eius igne comburunt. Et cum etiam puer aliquis infirmatur predicto modo faciunt idolum, et ligant super lectum ispius. Duces, millenarii et centenarii hyrcum semper habent in medio stationis.

Predictis vero idolis offerunt primum lac omnis pecoris et iumenti. Et quando primo comedere vel bibere incipiunt, primo eis offerunt de cibariis vel de potu. Et quando aliquam bestiam interficiunt, offerunt cor idolo, quod est in curru, in aliquo cipho, et usque mane dimittunt, et tunc auferunt de presentia eius et decoquunt et manducant. Primo etiam imperatori fecerunt idolum quod ponunt in curru ante stationem honorifice, sicut vidimus ante ordam imperatoris istius, cui offerunt munera multa; equos etiam offerunt ei, quos nullus audet ascendere usque ad mortem. Alia etiam animalia eidem offerunt; que si occidunt ad manducandum, nullum os confringunt ex eis sed igni comburunt. Ei etiam ad meridiem tamquam Deo inclinant, et inclinare faciunt aliquos nobiles qui se reddunt esidem. (Daffinà, Lungarotti, Menestò, Petech 1989, 236-7)

Alcuni dettagli di questa descrizione sono comuni all'Itinerarium di Guglielmo di Rubruck (II 7-8), che si differenzia da Giovanni da Pian del Carpine soprattutto per il tono «irridente»: per designare il feticcio si usano termini sostanzialmente spregiativi come puppa (bambola, pupazzo) e statuuncula (non statua!), e l'idolo più potente, quello al centro della sala, è descritto come una bambola paruula et (?) macilenta (Chiesa 2011, 349):

Et super capud domini est semper una ymago quasi puppa uel statuuncula de filtro, quam uocant fratrem domine, affixe parieti; et superius inter utramque illarum est una paruula, macilenta, que est quasi custos totius domus. Domina domus ponit ad latus suum dextrum ad pedes lecti in eminenti loco pelliculam hedinam impletam lana uel alia materia et iuxta illa, statuuncula, paruulam respicientem uersus famulas et mulieres. Iuxta hostium ad partem mulierum est iterum alia imago cum ubere uaccino, pro mulieribus, que mungunt uaccas: de officio enim feminarum est mungere uaccas. Ad aliud latus hostii uersus uiros est alia statua cum ubere eque, pro uiris, qui mungunt equas.

Et cum conuenerunt ad potandum primo apergunt de potu illi ymagini que est super capud domini, postea aliis ymaginibus per ordinem. Postea 
exit minister domum cum cipho et potu et spargit ter ad meridiem, qualibet uice flectendo genu, et hoc ad reuerentiam ignis; postea ad orientem, et hoc ad reuerentiam aeris; postea ad occidentem ad reuerentiam aque; ad aquilonem proiciunt pro mortuis. (Chiesa 2011, 24-5)

Infine, un breve accenno si trova in Odorico da Pordenone, nel capitolo XXXVI della Relatio, dedicato all'energica azione dei frati minori nella «magna Tartaria»:

Tunc fratres sua idola de feltro accipientes, que ipsi habent, cum cruce et aqua benedicta illa portant ad ignem; deinde omnes de contrata deos suorum vicinorum veniunt videre comburi. Tunc fratres, illa idola accipientes, illa ponunt in ignem et tunc illa de igne exeunt, propter quod fratres postea accipiunt de aqua benedicta, quam in ignem proiciunt, et statim demon fugit ab igne; et sic fratres idolum in ignem proiciunt, ibique comburitur. (Marchisio 2016, 221-2)

Un'ampia documentazione è fornita anche dagli esploratori europei che visitarono queste regioni tra il XVIII e il XX secolo, fra i quali si citano Pallas (1794), Erman (1850), Radloff (1883) e Zelenin (1936), e. Sulle funzioni degli ongyod vedi inoltre Roux de Rochelle (1990, 274-8); Tucci, Heissig (1970, 359-62); Atwood (2004, 423-4); Doerfer (1963, 179-81). Agli ongyod venivano - e vengono tuttora - offerte aspersioni rituali di kumiss, latte di giumenta fermentato; i primi pezzi di cibo durante i pasti; il primo latte delle greggi e delle giumente, ecc., come osservano i viaggiatori medievali nelle testimonianze appena riportate, e come si legge anche nella Storia Segreta dei Mongoli (cap. 193; de Rachewiltz 2006, 37 e 115), dove lo stendardo di guerra viene consacrato con libagioni prima della battaglia. Per tali aspersioni rituali, documentate nella Yuanshi, cronaca della dinastia Yuan, nella sezione relativa alle tradizioni nazionali e agli antichi riti, (cf. Ratchnevsky 1970).

Le indagini sul campo confermano la persistenza di questa pratica: mi diceva Bat - membro della comunità turcofona Dukha ${ }^{16}$ del nord della Mongolia - che lo eeren (forma tuvina per ongyon) è ciò che loro hanno di più prezioso, a cui si rivolgono in presenza di difficoltà di ogni genere. Allo eeren vengono sempre offerte aspersioni rituali. ${ }^{17}$

16 I Dukha sono una piccola etnia turcofona, circa 500 persone, che vivono nelle regioni settentrionale della provincia Khövsgöl in Mongolia. Circa la metà vive ancora allo stato nomade nella taiga e alleva le renne. Per la lingua e storia dei Dukha, cf. Ragagnin 2011.

17 Per una descrizione del cosiddetto sciamanesimo siberiano cf., fra gli altri, Radloff 1893, 1-67; Morgan 1997, 50-3; Harva 1938; Tucci, Heissig 1970; Roux 1990. Fra le popolazioni mongoliche le pratiche sciamaniche si sono meglio conservate fra i Buriati e i Mongoli Darkhat, stanziati nella regione Khövsgöl nella Mongolia settentrionale; cf. Badamxatan 
Il teonimo poliano «Nacigai» sembrerebbe insomma riprodurre la forma medio-mongolica Načigai descritta supra.

Tuttavia la sua maschilità e il fatto che «Nacigai» abbia moglie e figliolanza sembrerebbero frutto di confusione da parte del viaggiatore veneziano, dettata probabilmente da sfumati ricordi di varie forme di ongyod che aveva visto presso i ta(r)tari oltre che, forse, dalle informazioni che aveva ricevute su simili usanze e credenze animistiche di popolazioni limitrofe ai Mongoli dove, forse, veniva venerata una divinità terrena maschile.

A questo proposito vogliamo ricordare che nel pantheon siberiano esistono delle divinità maschili la cui descrizione corrisponde a un "Tellus Pater' (cf. Harva 1938, 243 e 247). Gli Altai della Siberia meridionale, ad esempio, credono in Ülgän o Bai Ülgän, un Dio del bene, antagonista di Erlik, il Dio degli inferi (Verbickij 2005, 412b). Eliade (1964, 198) puntualizza che Ülgän, benché dotato di attributi celestiali, non è del tutto una suprema divinità uranica. Egli è distinto da Tenggri - il Dio-Cielo, supremo Dio uranico delle popolazioni turco-mongole, che nella lingua Altai è Tengere Kairakan $^{18}$ - e possiede piuttosto le caratteristiche di un Dio dell'atmosfera e della fertilità, e ha una consorte e una figliolanza numerosa. La sua funzione è di proteggere la fertilità delle greggi e l'abbondanza dei raccolti.

Si noti infine l'informazione etnografica di Palladius $(1876,15-16)$ citata in Rockhill $(1900,60)$ riguardo agli idoli dei Mongoli daguri e bargu:

the Dahurs and Barhus have in their dwellings, according to the number of the male members, puppets made of straw, on which eyes, eyebrows, and mouths are drawn; these puppets are dressed up to the waist. When someone of the family dies his puppet is taken out of the house, and a new puppet is made for every newly born member of the family. On New Year 's Day offerings are made to the puppets, and care is taken not to disturb them by moving them, etc., in order to avoid bringing sickness into the house.

In conclusione, alla luce di quanto esposto, le informazioni che il Devisement dou monde fornisce su «Nacigai» sembrerebbero riflettere in un'unica divinità vari aspetti del complesso mondo spirituale dell'Alta Asia del XIII secolo, i cui molteplici tasselli non ci sono noti data l'assenza di fonti accurate a riguardo. Tale poliedricità è però documentata, come brevemente esposto in questo saggio, da viaggiatori, etnografi e altri studiosi che a partire dal XIII secolo esplorarono le lontane terre della Russia

1986; Krader 1954, 1975, 1978; Žamcarano 1909; Shimamura 2014; Purev, Purvee 2010; Diószegi 1963.

18 Il termine kairakan è di origine mongolica: cf. mongolo letterario qayiraqan «merciful, gracious; sacred mountain; a formula introducing or concluding a prayer» (Lessing 1995, 914a). 
siberiana e della Mongolia. Benché temporalmente molto più tarde, tali testimonianze, soprattutto quelle che riguardano le popolazioni meno influenzate dal buddismo, sono di grande importanza per meglio capire le informazioni e i fraintendimenti di Marco Polo.

Figura 1. Idolo di stoffa (eeren) Dukha (foto di Elisabetta Ragagnin)

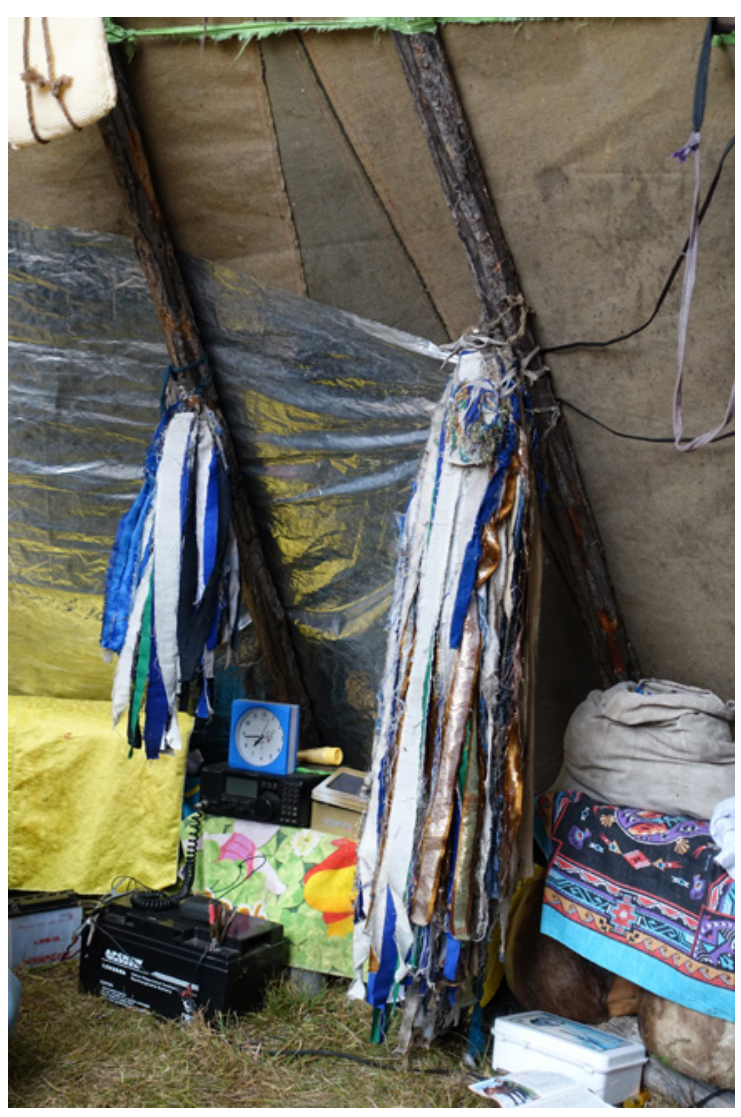




\title{
«Reverendo signore mio» Note sulla lingua di Ramusio dalla corrispondenza con Pietro Bembo
}

\author{
Fabio Romanini \\ (Università degli Studi di Trieste, Italia)
}

\begin{abstract}
The essay investigates G.B. Ramusio's language in his letters to Pietro Bembo (1537-38) preserved in the Ms. Ambrosianus D 335 inf. and recently published by A. Del Ben. This corpus is the largest known amount of autograph texts written by the Venetian official and gives a rare insight into the tangled web of correspondents and into the fast communication links that lead to Venice from various sources. Compared to Ramusio's dissertations and introductions to the exploration accounts published in the first volume of his Navigationi et viaggi (editio princeps 1550), these texts are quite more colloquial: the linguistic analysis shows all the features of a lower register and lets many local words and phrasemes appear to the surface of the page.
\end{abstract}

Sommario 1 Introduzione. - 2 II corpus manoscritto delle lettere di Ramusio a Bembo. - 3 Alcuni dettagli di contenuto: la politica. - 4 La vita veneziana. - 5 Spoglio linguistico. - 5.1 Grafia e fonetica. - 5.2 Morfologia. - 5.3 Lessico. - 5.4 Sintassi e testualità. - 6 Riepilogo.

Keywords G.B. Ramusio. Linguistic analysis. Letters. Venetian dialect. Pietro Bembo.

\section{Introduzione}

Licenziando alcuni anni fa il mio studio monografico dedicato alla lingua di Giovanni Battista Ramusio nelle Navigationi et viaggi avevo deciso di inserire, in appendice, uno spoglio linguistico delle dissertazioni e dei discorsi introduttivi del curatore contenuti nel primo libro dell'opera (in base alla versione della princeps giuntina del 1550). La scelta dei testi si basava su una premura di 'autorialità': in mancanza di carte autografe dell'impresa, il primo libro offriva elementi di relativa sicurezza rispetto al controllo che Ramusio poteva avere esercitato sulla tipografia (mentre per il secondo libro, pubblicato postumo nel 1559, e per il terzo, del 1556, non si può nutrire, allo stato attuale delle indagini, altrettanta fiducia nell'originalità della veste linguistica). Lo spoglio voleva costituire una prima verifica della qualità della lingua di Ramusio, fondata su testi di discreta estensione (Romanini 2007, 257). 
Nel 2006 Andrea Del Ben ha pubblicato uno studio intitolato Giovanni Battista Ramusio cancelliere e umanista, in cui ha curato l'edizione di 45 lettere di Ramusio a Pietro Bembo, con il corredo di una nota linguistica (uno spoglio, seppure succinto, di tipo tradizionale, con osservazioni di grafia, fonologia e morfologia). La disponibilità di tale lavoro aveva allontanato l'urgenza di ulteriori verifiche; ma a distanza di qualche tempo mi è ora possibile integrare quello spoglio e proporre qualche nuovo rilievo, soprattutto di tipo testuale, sul pur esiguo corpus di epistole che si è conservato. A tutt'oggi peraltro, salvo un'eccezione, non sono disponibili edizioni dei pochissimi altri autografi noti di Ramusio. ${ }^{1}$

Come ricostruiscono Donattini (1980 e 1986) e lo stesso Del Ben (2006), Ramusio era stato un uomo di fiducia della tipografia aldina, prima dello stesso Aldo (di cui fu anche, assieme ad altre persone, esecutore testamentario) e poi del suocero, e in seguito aveva frequentato per decenni la tipografia dei Giunti. Era considerato fin da giovane uno studioso esemplare per acribia e capacità di valutare manoscritti e opere a stampa: lo testimoniano quattro lettere di Navagero conservate alla Biblioteca Marciana, datate tra il 1510 e il $1515 .^{2}$ Il giovane Ramusio aveva condotto studi su opere latine e greche in periodi appena precedenti alla loro pubblicazione a stampa (sono i casi di Galeno, di Polibio, ecc.); e anche se per esse non è indicato il nome del curatore, questi dati sollecitano la verifica di tale ipotesi attributiva. Sarebbero insomma tanti i dati biografici che collegano precocemente Ramusio e le imprese di stampa: sufficienti, per lo meno, a sospettare che la versione linguistica delle Navigationi et viaggi sia stata sorvegliata non distrattamente dal loro curatore. Del Ben (2006, XXVIII) segnala, tra le lettere di Fracastoro a Ramusio, una epistola datata 25 gennaio 1548 in cui il geografo veronese commenta lungamente alcuni testi che ha ricevuto dall'amico, e soprattutto quello sul corso del Nilo, concludendo in questo modo:

bisogna pur empir il foglio, non havendo cose degne et rare, come sono quei bei Viaggi, i quali se farete stampare con l'altre geografie, oltre

1 Nel ms. Marc. It. VII 163 (= 7653) sono di mano ramusiana il breve indice per materie all'inizio, alcune note sui fogli di guardia finali e alcune brevi postille al testo, senza nessuna indicazione di data; nel Marc. It. VII 1820 (= 8422) è di mano ramusiana, a c. 3r (ma n.n.) l'Instrumento d'acquisto della possession della Spesa del 15 maggio 1525; si conta un'altra lettera a Bembo, datata 26 febbraio 1525, conservata nel ms. Ital. C 23 della Bodleian Library di Oxford (c. 115r-v; è segnalata da Clough 1967-72, 33); infine Andrea Del Ben ha pubblicato una lettera di Ramusio a Carlo Gualteruzzi del 17 settembre 1547 (Del Ben 1994-95, 207-8), e cita un'altra lettera autografa del 21 agosto 1543 (Del Ben 2006, XXXVIII nota 111: sarebbe «già citata», ma non ne trovo traccia nel testo).

2 Le lettere, apografe, sono relate dal ms. Marciano Italiano X 143 (=6335): sono datate 21 dicembre 1510, 10 maggio 1514 e 13 e 17 gennaio 1515 (questa ultima senza indicazione di anno). Furono pubblicate in Cicogna 1853, 322-5 e sono analizzate più in dettaglio da Del Ben 2006, XX-XXI. 
all'utilità che darete $\mathrm{al} \mathrm{mondo,} \mathrm{ciascun} \mathrm{ne} \mathrm{riceverà} \mathrm{tanto} \mathrm{piacere} \mathrm{quanto}$ di cosa già gran tempo stampata. (Lettere 1560, 730-1)

Il progetto delle Navigationi et viaggi doveva essere ormai delineato, e molti materiali preparati: Fracastoro si congratulava con l'amico per il divertimento che gli offriva con i racconti di viaggio e i testi geografici. ${ }^{3}$

Tornando però al corpus delle lettere a Bembo, è necessario aggiungere qualche cautela prima di procedere ai rilievi più tecnici. È assai suggestivo che il destinatario delle 45 epistole sia Pietro Bembo; ma lo sarebbe ancora di più se questo Pietro Bembo fosse il teorico della lingua italiana, l'autore della proposta decisiva per la soluzione della questione della lingua, l'autore delle Prose. Così, invece, non è: il destinatario è piuttosto l'amico Pietro Bembo, che Ramusio potrebbe avere conosciuto presso la tipografia aldina, visto che la più antica lettera che possediamo (di Bembo a Ramusio) è datata 18 dicembre 1508 (Travi 1987-93, 2: nr. 282), e non sembra l'inizio di una corrispondenza (peraltro risponde a una precedente di Ramusio, ringraziandolo per le informazioni ricevute). Anche se Bembo è di quindici anni più vecchio di Ramusio, e le lettere mostrano un rapporto apparentemente formale (Ramusio indirizza le lettere «al reverendo signore mio il signore messer Pietro Bembo prior de Ongaria»), i due si chiedono reciprocamente favori senza attardarsi in questioni di etichetta. Ramusio è uomo di fiducia di Bembo a Venezia quando questi si rifugia negli otia della villa padovana: è persona capace e ben introdotta, seppure di classe sociale inferiore a quella del futuro cardinale, nonché storiografo della Repubblica. Peraltro, dopo che Bembo scampò all'avvelenamento, nel 1530, fu proprio Ramusio, conoscitore dei veleni e dei loro effetti (di botanica in generale, e anche di ittiologia e di antiquaria, come emerge dalla corrispondenza con Navagero degli anni '20), a insistere affinché le magistrature della Serenissima indagassero sul caso. ${ }^{4}$ Quando Bembo, subito dopo, divenne bibliotecario della Nicena, fu piuttosto Ramusio a svolgere il ruolo di custode fino al 1543 prima, e poi ancora fino al 1547 affiancando Bartolomeo Ramberti. Insomma, Ramusio è funzionario e persona fidata: è a lui che Bembo si rivolge quando viene informato che il suo Della lingua volgare (e cioè il testo oggi noto come Prose della volgar lingua) è stato oggetto di plagio, affinché i colpevoli siano perseguiti e puniti duramente; è la persona a cui Bembo affida il compito di comprargli una casa all'asta, dandogli istruzioni precise. Ma Ramusio, come testimoniato da varie mis-

3 Più che un «invito», come scrive Del Ben, credo che possa trattarsi di un augurio entusiastico, visto che la lettera è scritta piuttosto a ridosso della stampa (la princeps del volume I delle Navigationi fu pubblicata dai Giunti nel maggio 1550).

4 Lo ricorda Del Ben 2006, XXXV-XXXVI, citando due lettere di Pietro a Giovan Matteo Bembo, datate 8 e 18 settembre (Travi 1987-93, 2: nrr. 1140 e 1148). 
sive inviate da Bembo, manda all'amico qualche 'allegato' gradevole («vi ringrazio delle seme di melloni, benché a ringraziarvi de tutte le vostre amorevolezze la penna non basta»: Travi 1987-93, 2: nr. 472; «semi di lattuca»: Travi 1987-93, 2, n 644; «accetto molto volentieri i vostri calmi de vite... accetto similmente gli sparigi, i quali mi saranno sopramodo cari; e anco di questi ne vorrei gran quantità»: Travi 1987-93, 2: nr. 732; «due vasi: uno de ulive molto bone, e l'altro di fenocchi ottimi»: Travi 1987-93, 2: nr. 851). Bembo però approfitta della sua posizione rilevata anche per rivolgere qualche rimprovero all'amico: «Del non mi aver voi voluto dir cosa alcuna degli Asolani è stato un dir assai, cioè che e' non ve sono piaciuti. Pazienza» (Travi 1987-93, 2: nr. 472).

\section{Il corpus manoscritto delle lettere di Ramusio a Bembo}

Il codice Ambrosiano D 335 inf., composito di soli fogli cartacei, risalente alla prima metà del sec. XVI, consta di 83 cc. numerate, legate; la legatura misura mm $335 \times 239$, i fogli sono di varie dimensioni. Avvolgono il ms. due fogli di guardia cartacei, più altri due di recente restauro. La coperta attuale è moderna, ma è stata conservata anche una rilegatura più antica. ${ }^{5}$ Contiene 45 lettere di Giovanni Battista Ramusio a Pietro Bembo (cc. 2-74 e 79-83), più una a Bembo della Morosina datata 25 febbraio 1525, a c. 1; una nota di mano di Bembo e da lui segnata «mezzo luglio MDXXXIX» a c. 75 (a c. 76 la busta); una lettera di un Querini da Venezia al Bembo datata 5 agosto 1539, a c. 77r-v. Le lettere di Ramusio sono tutte scritte da Venezia. La più antica è del 9 luglio 1537, la più recente del 27 ottobre 1538. Le ultime tre (quelle raccolte in fondo al codice) recano il giorno, ma non l'anno: nell'edizione Del Ben (2006) sono state datate e inserite tra le altre in ordine cronologico. La qualità della scrittura è generalmente molto corsiva: alcune lettere sono comunque ben leggibili, mentre altre sono state scritte molto in fretta, alcune con una penna in cattivo stato. In particolare le formule di congedo e la firma sono spesso piuttosto rapide; la trascrizione di Del Ben, piuttosto corretta vista la difficoltà di lettura, non soffre comunque di fraintendimenti decisivi. ${ }^{6}$

5 Per una descrizione dettagliata del codice si veda Del Ben 2006, LI-LIII.

6 Rispetto all'edizione, sono da correggere almeno i seguenti luoghi: medesimo $>$ medemo 1.6 (si indicano numero di lettera e rigo progressivo); marcadanti $>$ marcadantie 2.14; forono > fono 4.6; Iddio ne mandi > Idio ne ne mandi 8.17; conte > conto 8.32; entendono $>$ intendeno 12.12; questo tal generalio > questa tal generation 13.20-1; partito > parté 15.2; montarono, andarono > montarano, andarano 18.11-2; medesima $>$ medema 22.5; tornarono > tornorono 22.15; vostra signoria > signoria vostra 22.17; Gurirasco > Guayrasco 23.13; andato in Zante $>$ andato verso il Zante 32.3-4; medesima > manco 32.22; può > puol 32.22; certo > Carlo 32.23; mudarvi > mudarmi 33.3; quegli > questi 33.25; gravissimi > grandissimi 35.17; andarono > andarano 38.22. L'edizione interpretativa muta in ghe tutti i ge 
Le lettere del codice Ambrosiano seguono uno schema fisso: informazioni su Venezia seguite da notizie sulla politica romana e poi internazionale (movimenti dei Turchi, avvenimenti in terra francese, ecc.). Bembo, dalla sua villa padovana, era decentrato rispetto alle vie di scambio dei dispacci e degli ambasciatori, e perciò rischiava di perdere aggiornamenti, o di riceverli in ritardo: ed era essenziale per lui essere continuamente aggiornato sullo stato di salute dei cardinali, o sui rapporti tra la Francia e l'Impero. Ramusio gli scrive assai di frequente, addirittura in certi casi a giorni alterni, e in cambio chiede a Bembo la cortesia reciproca: una maggiore frequenza delle sue epistole, facendogli garbatamente osservare che i 'cavallari' viaggiano quotidianamente. Peraltro sono andate perdute molte epistole anche relative a questi stessi mesi di corrispondenza: lo si intuisce dalla frase: «altro non li ho a dir havendo heri sera scrittoli» (lett. 42 del 23 dicembre 1537), che si riferisce appunto a una epistola del giorno precedente, non conservata all'interno del corpus ambrosiano.

La natura privata di queste lettere fa sì che la loro tipologia testuale sia assai diversa da quella dei testi delle Navigationi, destinati a un pubblico più allargato. Esse rispecchiano, nella lingua, l'uso del veneto colloquiale che Bembo stesso mostra nelle proprie epistole a 'Zuan Batta'. È dunque difficile confrontare testi destinati a uso pubblico e lettere private, in cui l'opzione di registro è ovviamente colloquiale. Tuttavia, se è tutto sommato condivisibile, come ha osservato Lorenzo Tomasin $(2001,142)$, che la cancelleria veneziana riesca a esprimere un «italiano pressoché perfetto», è però vero che anche nei testi ufficiali dei funzionari alcuni aspetti fonomorfologici non siano adeguati alla norma. Più di altri, fenomeni come l'apocope (degli infiniti in particolare, o dei suffissati in -zione), la mancanza di anafonesi e la morfologia verbale settentrionale resisteranno ancora per tutto il secolo e oltre alla normalizzazione laddove i correttori operanti in stamperia siano meno attenti a questi aspetti. Come scrivevo, Ramusio è assai più bravo a correggere i testi altrui che a usare forme anafonetiche nei propri; ma è anche vero che le ristampe delle Navigationi et viaggi compiute poco dopo la morte del curatore mostrano un ritorno di forme senza anafonesi anche nei punti in cui la forma era stata normalizzata sul fiorentino.

\section{Alcuni dettagli di contenuto: la politica}

L'incipit delle lettere è sempre lo stesso: il segretario veneziano chiama il più anziano amico «reverendo signore mio», e si firma come «servitor

grafici, e inoltre scioglie sempre l'abbreviazione per indicare il luogo (Venetia; ma quando è scritto per esteso è sempre Venexia) e la firma (Rham con svolazzo abbreviativo; per esteso è Rhamusio o Rhammusio e non altro). Altre minuzie riguardano grafie e piccole differenze fonetiche. 
Rhamusio»; Bembo chiama invece Ramusio «onorando quanto fratello»e talora si firma «Bembus tuus» o «Bembus frater».

Nella prima parte di ciascuna lettera Ramusio aggiorna Bembo sulle notizie che arrivano da Roma tramite i 'cavallari' (o 'corrieri'), in particolare se ci sono notizie importanti sull'avanzata dei Turchi. Ramusio spesso attende fino a ora tarda prima di mandare le lettere, e scrive anche a brevissima distanza dalla missiva precedente, se è giunta una novità rilevante. Ad esempio:

in questa hora 24 è gionto un corriere. Parté da Roma già dui dì, et dovea esser questa matina qui, che ne avisa come se hanno lettere da tutte le marine de Puglia de 11 che l'armata turchescha, nel ditto giorno, a bon hora si levò tutta con tutte le genti et cavalli, et andavan verso Levante incolfandosi. Questo sono precise le parole. [...] Questo aviso de così gran momento non ho voluto restare de dirgelo. (lett. 15 del 21 agosto 1537)

Seguono poi notizie di politica italiana:

il par che un di questi dì passati il signor Hippolito da Corezzo, figliol de quella de Gambara, sia andato con 700 fanti et 200 cavalli a Bozolo et intrato dentro l'ha sacheggiato et fatti pregioni tutti. L'ha vergognate tutte le donne et il signor Cagnin [Gianfrancesco Gonzaga], con la donna et sorella, a gran pena sono fuggiti, et ditto signor Hippolito dice haverlo fatto de ordine de signor marchese del Guasto.

Il signor Alessandro Nuvolara, qual stava con Francesi, è sta' morto intrando in Pinarol da un schioppo. Par che tutte le genti di Francesi siano fra loro alle mani.

In Fiorenza è sta' tagliata la testa a Baccio Valori et ad un suo figliuolo et un altro fiorentino che mi ho domenticato il nome [Antonfrancesco degli Albizzi]. Messer Philippo Strozzi vogliono mandar a star nel castel de Milano. (lett. 17 del 26 agosto 1537)

Ramusio è sempre molto edotto delle ultime novità, e scrive quasi anticipando le breaking news dell'epoca:

Questi signori hanno deliberato di tuor molte terre qui in la Schiavonia che li sono de impedimento, come è Obrovaz, Scardona et altri lochi, et già li clarissimi generali dieno a questa hora esser sopra il fatto e presto se ne saperà la executione. (lett. 29 del 28 settembre 1537)

Abbiamo anche notizia dell'inizio di una nuova fase difensiva di Venezia, perché l'amministrazione aveva avuto dimostrazione di quanto fosse inadeguata la struttura dello Stato da Mar a fronte dell'offensiva turca: 
Questi signori mandano maestro Michel, nostro inzegner, con infiniti valenti maestri, cioè capi, per fortificar Corfù come ha disegnato il signor duca [Michele Sammicheli]. (lett. 33 del 16 ottobre 1537)

Un mese prima, infatti, si era aperto il conflitto, e la porzione di epistolario conservata ne dà testimonianza (è probabilmente la notizia più importante di tutto il mucchietto):

Heri ad hore 23, che sia con il nome del Spirito Santo, habiamo tolto la guerra aperita con il Turco et sarà una lega papa, imperatore et Signoria. (lett. 23 del 15 settembre 1537)

Qualche notizia è riservata: «Questa parte che li scrivo del Canal la signoria vostra la tengi apresso di sé» (lett. 18 del 28 agosto 1537, nota inserita dopo la firma). Ciascun resoconto è di solito concluso dalla frase ricorrente «et non si sa altro». Ci sono talvolta anche commenti alle notizie, che possono riportare la vox populi:

Questo è tutto il riporto che manda il signor viceré, al qual per homini savii non se li dà piena fede et se stupisseno come essendo stato dentro el non habbi portato fuori lettere dei rettori. (lett. 28 del 26 settembre 1537)

A riprova della velocità con cui Ramusio fa rimbalzare le notizie viene anche una nota dell'apparato di Del Ben (2006) per la lett. 29:

sono gionte lettere da Roma de 26, che portano lettere da Corfù de 12 et 16 [...] narrano tutti in conformità che Nasbassà era stato a vedere il sito de Corfù. (lett. 29 del 28 settembre 1537) (corsivo aggiunto)

Stato è correzione interlineare di venuto, che sarà stata la lezione dell'antigrafo da cui Ramusio sta velocemente copiando la notizia per inoltrarla all'amico, trascurando inizialmente di cambiare il punto di vista. Un esempio interessante del funzionamento di questi collegamenti postali è l'incipit della lett. 11:

heri sera ad una di notte venero lettere da Roma de 8, per le qual si manda una relation mandata al conte de Agillar, oratore cesareo, dal marchese de la Tripalda de 2 de avosto, come un Bartholomeo de Brande da Chioza refferiva che alli 27 de luglio, il venere, il capitano general si levò da Corfù... (lett. 11 dell'11 agosto 1537)

Si vede da qui molto bene la traccia dei dispacci e dei racconti che muove in direzione di Venezia, attraverso punti intermedi, e da Venezia poi riparte verso nuovi destinatari. Ancora un esempio: «Questa matina vi son lettere 
da Roma de 4, di Napoli de 2, da Ottranto de 28 luglio» (lett. 8 del 6 agosto 1537). Bastavano dunque due giorni per l'arrivo della posta da Roma.

In generale, sarà da notare la struttura informativa molto replicata delle lettere, che pongono in prima posizione il tema - un argomento già illustrato in una missiva precedente - accompagnato dagli aggiornamenti, che talora possono essere anche clamorose smentite:

Del capitano Cocianer del re de Romani che era in la Schiavonia contra Turchi, et fo ditto che li havea rotti, par che non si confirma cosa alcuna. (lett. 34 del 21 ottobre 1537)

\section{$4 \quad$ La vita veneziana}

Dei minuti argomenti di vita pratica, tra i quali sono frequenti richieste di riscossione di crediti e di pagamento di debiti, o richieste a Bembo per sollecitare il fattore a comunicare le ultime novità, credo che possano essere maggiormente interessanti le questioni culturali, e più curiosi $i$ bisogni pratici di Ramusio. Raccolgo qualche citazione sui libri richiesti da Bembo, e cercati da Ramusio sul mercato:

io mando la Pedia de Ciro di Xenophonte di quelli stampati de Aldo, di quali per non se ne trovar più non vogliono dui scudi d'oro et ancor con difficultà. Item li Epigramma greci, lire 3. Del vocabulista latino et greco stampato in Alemagna, che son li migliori stampati fin al presente, non se ne trova alcun in Venetia. Io ho domandato a tutte le boteghe se vendeno quelli di Aldo, ma non hanno la metà di vocabuli respetto a quelli di Alemagna. (lett. 6 del 4 agosto 1537)

Ancora: «Delli Livii in $8^{\circ}$ stampati in Lion non se ne trova più, tuti sono sta' spazzati» (lett. 26 del 22 settembre 1537). Nella corrispondenza tra i due si parla anche di manoscritti ambiti:

Mi ha ditto messer Tomaso Gionta come è venuto a casa de vostra signoria qui in Padoa un secretario del reverendissimo Salviati qual ha amicitia grande con un don Ottavio da Ferrara che è quello che ha tutte le correttioni de le Orationi de Cicerone, de le qual ne monstrai due a vostra signoria. De gratia quella li domanda come se potrian haver et me ne dia aviso una parola de quanto la signoria vostra potrà intender da costui. (lett. 45 del 27 ottobre 1538)

Tra i libri, si affaccia l'argomento geografico in un paio di occasioni: «ho fatto che messer Carlo [Gualteruzzi] manda a vostra signoria un Corfù ben fatto in Roma» (lett. 34). Ramusio si riferisce a una carta geografica: 
Bembo gli richiede infatti carte geografiche aggiornate e dettagliate delle zone di guerra; poi invece ringrazierà Cola Bruno, che evidentemente gli procurò la carta di Corfù, e gli chiederà anche «la Toscana e il Piemonte o Savoia, che intendo essere state impresse costì molto bene» (Travi 198793, 4: nr. 1893). E inoltre, tornando alla 'voce' di Ramusio:

Voria saper da vostra signoria se mai l'ha veduto una epistola de l'episcopo Nidrosiense, che è in Gotthia, qual fu scritta a Papa Leone del 1520 , dove si narra tutte le cose admirabili che sono sotto la tramontana in que' paesi; de gratia vostra signoria pensi se mai l'ha veduta over se per caso ne l'havesse la copia et me ne dia aviso. (lett. 44 del 25 ottobre 1538$)^{7}$

L'interesse per i libri coinvolge anche gli affari nuziali. All'inizio del periodo testimoniato nelle lettere Ramusio ha speranze di sposare la figlia di Febo Capella, altro funzionario della Serenissima, e scrive a Bembo che è in trattative per avere in dote un libro particolare: «io ho con il nome de Dio concluso con il Capella, et giovedì li tocharò la mano; et ho parlato del libro del Petrarcha che voglio in conto de dote» (lett. 8 del 6 agosto 1537). Proprio sulle nozze, il cui contratto si interruppe il giorno seguente, Ramusio sfoga la sua delusione con l'amico:

Reverendo signore mio, gran cosa è qualche volta li augurii che si ha nel scrivere come è sta' questo di vostra signoria nella lettera sua de heri, che ralegrandosi de le nozze mie li venne fatte queste parole anchora che non fatte e le depennò. Che così è stato, che havendomi loro promesso di darmi al tochar de la man ducati 300 de contadi io li feci intendere che io non li voleva far acontar et che li dovesseno mettere in un banco di Prioli, et instandoli sopra di questo fu trovato che re vera non hanno de contadi un soldo, sì che io non l'ho voluta andar a vedere, et anchora che loro habbino ditto di volerli trovar fra termine de 8 dì, io non la voglio più per conto alcuno et voglio veder in che stato sarà il mondo. Sì che prego vostra signoria non ne dichi altro, et sia contenta di mandar a chiamar el mio Carlo et dirli che ho havuto li ducati 22 et che circa che li havea scritto di havere preso moglier non è alcuna cosa et che io bertizzava, acciò che 'l non vadi conquendo il mondo. (lett. 10 dell'8 agosto 1537; sottolineatura nell'originale)

Sfumato il negozio, la preoccupazione successiva di Ramusio è per la sua casa padovana, che viene danneggiata da una forte grandinata: «Mi

7 Donattini $(1980,87-9)$ scrive che Ramusio vide probabilmente questa lettera grazie ad Alvise Da Mula. 
è sopragionta la tempesta in li copi de la mia casa de Padoa che non mi bisognava altro. Idio ne agiuti» (lett. 13 del 18 agosto 1537). Soprattutto, il problema maggiore sarà la riparazione dei vetri. Dapprima Ramusio chiede a Bembo di sovrintendere ai lavori, e la conclusione del discorso sfocia in una ironia leggera:

vostra signoria sia contenta di mandar qualche persona delli suoi a vedere non so che vedri che dicono bisognar rifar da novo, che la tempesta mi ha rotto sopra la sala de la mia casa et con quella manco spesa che la puol farli conzar. Item pagare lire 17 [...]. Item far andare il suo fattore [...] a pagar [...]. Item pagar le daie di questo anno [...]. Item le daie in nome mio [...]. Vede vostra signoria che se la mi ordina un servitio io ge ne do X al tratto: quella mi perdoni et a lei mi raccomando. (lett. 32 del 4 ottobre 1537)

In seguito, però, le spese ingenti strapperanno più di un lamento a Ramusio:

Delli vedri de la casa quel homo da ben che sta dentro ha pensato che la casa sia di vostra signoria et così il fattor ha havuto in animo di conzar case sue [cioè di Bembo] et non de un pover homo come son io, che sono andati cercando a loco per logo tutti li busi rotti. La mità di questi vedri, quelli che stanno dentro, li hanno rotti loro et non la tempesta. Basta, bisogna haver pacientia. Almanco vostra signoria mi faci questo servitio di mandar il fattor a tuor in nota di sua mano tutte le vedriate che sono conze, perché al partir questi poltroni 4 mesi avanti andarano a Montagnana et mi lassevano tutta la casa sottosopra et rotti più che non sono adesso. Io l'ho conza' 4 volte con simel persone, non voria almancho per altri anni conzarla, se non intravenisse tempesta. Ma conzarli a' poltroni che per sua poltronia li fanno romper. Io mi despiero. Io voglio haver una nota sopra un foglio autentica che mi lassino li vedri come io li ho conzi, et se li do fastidio mi perdoni. (lett. 38 del 9 novembre 1537)

Infine, un'altra preoccupazione di Ramusio riguarda la presenza in casa di una donna di servizio, evidentemente necessaria per sopperire alle esigenze quotidiane dopo la scomparsa della madre (avvenuta poco prima, l'8 ottobre del '37; la moglie di Ramusio, Franceschina Navagero, era morta l'anno precedente):

et pertanto non posso far che non supplichi vostra signoria che se lì a Padova la mi potesse trovare una donna de tempo fidata al qual potesse raccomandar la cura di casa, qual tenisse sotto di sé una massara per far le cose de cusina, io non resteria per ogni danaro de scamparla per qualche tempo che la stesse con me, perché mi è forzo de accompagnarmi, non volendo fare al hostaria tutto il tempo de la vita mia. Di questa 
donna di grazia vostra signoria se ne informi et più presto che la potesse haver magior gratia la mi faria. (lett. 33 del 16 ottobre 1537)

Pochi giorni dopo, il problema è stato risolto: «vostra signoria non cerchi altra femina per governo de casa mia per haverne trovata una ottima et la rengratio de la faticha presa» (lett. 35 del 24 ottobre 1537).

\section{Spoglio linguistico}

\subsection{Grafia e fonetica}

La tipologia testuale e l'interlocutore condizionano la scelta di codice e di registro da parte di Ramusio. Affiorano infatti con frequenza tratti locali, indizio di una lingua poco sorvegliata. Per le grafie, pochi sono gli aspetti rilevanti, se non la persistenza di forme etimologiche come $h$-iniziale di parola o il gruppo ti più vocale, oltre a sporadici nessi consonantici non assimilati. Va almeno segnalato che $g$ ha valore velare nelle forme $g e 4$, passim e vengi 13. In qualche caso l'affricata dentale sorda può essere resa con ci, come in forcie 'forze' 35, pacientia 38, ecc.

Relativamente al vocalismo tonico, si trovano forme senza dittongazione, come novo (lett. 1), vol 1 (ma puol 32), heri 9, vode 'vuote' 11, boni 11 , fori 'fuori' 13 , fochi 21 , ecc., e viceversa altre con il dittongo, come Spagnuoli 3, 5, ecc. (ma anche Spagnoli 5), figliuol 5, ecc. oltre a forme indigene come despiero 6, tuor 18, ecc. Frequenti sono le forme prive di anafonesi, quali gionto 8 , congiongersi 8 , Marseglia 18, prencipe 23 - se non vedo male, unica occorrenza tra tanti principe - ecc., a cui aggiungo parole di formazione più recente, che presentano vocale tonica più aperta in analogo contesto: Fiamengi 1, Ongaria 1, ecc.

In atonia il gruppo -ar- è predominante nei futuri (tardarano 1, comprarò 4 , mandarò 4 , ecc., contro haverò 3 , manderò 3 , ecc.) e compare anche in altre parole: fantarie 1, marcadantie 2, artellaria 7 , hostaria 33, ecc. Di contro, in posizione postonica, lettera 1 e passim.

È ovviamente diffuso lo scempiamento consonantico (deti 'io diedi' 2, tocò 2 , sesanta 3, comesso 3, aviso 4, mezo 5, adosso 5, ralegrandosi 10 , venero 11, copi 13, mazo 15, corier 17, efetti 21 , obligato 23 , ecc.), con qualche ipercorrettismo come vella 1 , refferiva 11 , mulli 38 , ecc. Non stupisce nemmeno la frequenza con cui compaiono forme con lenizione (segondo 2, cargar e cargano 2 con sincope, avosto 4, sabado 11, saludar 11, anche vedri 32, ecc.) e con affricazione, sia di sorda, sia di sonora (Zuane 1, Franza 1, lanze 1 , zudese 3 , hozi 4, frezzata 4, Chioza 11 , perzò 13 , zanze 15, comenzata 18, rezimento 23 - ma regimento 24 -, zorni 25 , scaramuzze 30 , le zitae 'le città' 37 ecc.), e anche con assibilazione (tresento 2, brusò 4, cusina 'cucina' 33, busi 'buchi' 38, ecc.; con qualche oscillazione come pregioni 'prigionieri' 5 
e presoni 7). Per gli esiti di -ARIUS (e -ARIA) segnalo: cavallari 1, fornaro 31 e massara 33. L'esito di $\mathrm{D}+$ jod, oltre alla normale assibilazione, può anche essere agiuti 13 (analogo a quello di sola jod in Acquilegia 42).

Tra i fenomeni generali, caratteristica è ovviamente l'apocope, generalmente dopo sonorante (e tipicamente per gli infiniti): pension, recever, mandar, portar, general, scriver, par, delphin, Lion, ecc., e talvolta la caduta nei participi coinvolge l'intera ultima sillaba: era sta' 1, solo per limitare lo spoglio di questo fenomeno alla prima lettera. È soprattutto questa caratteristica a conferire alla lingua l'aspetto antifiorentino avvertibile anche a una lettura superficiale. Notevoli anche le metatesi di bergantin 'brigantino' 8 e di prede 'pietre' 11.

\subsection{Morfologia}

L'articolo determinativo maschile singolare è alternatamente il (il mio fattore 1) oppure, assai minoritario, el (el procurator 2), mentre al plurale Ramusio usa li (li reverendi padri 1), con possibilità di palatalizzazione (anche nelle preposizioni articolate: degli fanti 5). Qualche uso non è in linea con le indicazioni delle Prose: nel scrivere 10, un schioppo 17, del Spirito Santo 23, il stato 42, ecc. Altri invariabili di provenienza locale sono i numerali disdotto 1, vintidò 2, do 3 (ma anche dui 5). Notevoli anche i nomi dei mesi di luio 3, avosto 4, ottubrio 32 , novembrio 39, decembrio 42 (ma settembre 19, passim), il giorno di venere 11 (non è composto con dies).

Per i pronomi, è tipico il ge /ge/ 4, passim per 'a lui, a lei, a loro' (anche come enclitico: dirgelo 15), ma più spesso si trova li per il maschile (io li scrivo 1, non li ho a dir 2, dittoli 3, ecc.); alla prima persona si trova mi non solo come pronome atono (con $\mathrm{mi}$ 4). In posizione di soggetto si possono trovare anche lo, la e le, o il plurale maschile $i$ : l'è ben vero 2 , la vederà 4 , che le siano 12, vedendo che i hanno perso 40. In qualche caso Ramusio adotta la forma impersonale per riferirsi a un generico 'noi' che include tutti i veneziani: qui si sta molto sani 17, ancor non si hanno potuto haver risposta de Spagna de le lettere de la lega, et si sta in grandissima espettation 37. Il pronome obliquo di I plurale è ne: Idio ne ne mandi de bono 8 , ne lo haverian scritto 26, ecc. Caso particolare tra i possessivi è sui fratelli 9.

La morfologia verbale è, come è facile immaginare, il settore più esposto alle forme extrafiorentine, o perlomeno a forme toscane argentee che affianchino quelle settentrionali. Alla terza persona singolare trovo vidde 4 (pl. viddero 22), volse 14 (pl. volseno 22), conducé 18, ecc. Per la prima persona plurale trovo il tipico recevessemo 4 , anche se pare forma isolata entro l'epistolario. Ci sono numerosi casi di polimorfismo, come per dettero 1 e detteno 4 . Elenco alcune forme di terza plurale: per il presente dominano le forme settentrionali commetteno 2, dieno 'devono' 4, vendeno 6 , intendeno 12 , e per essere si trovano fono 4 e forono 7 ; per il 
passato remoto si alternano le forme analogiche (oppure toscane) in -(o) no e quelle con desinenza settentrionale -ano per la prima coniugazione e -eno per le altre: caminorono 5, scontrorono 14, tagliorono 14, scopersono 13 , andorono 14 , ordinorono 22 , e poi feciono 19 , mandòno 24 , ma anche affondarono 14, e inoltre montarano 18 , andarano 18. Per la coniugazione in -ire alle forme deboli modellate su quelle in -ere si aggiunge la desinenza settentrionale partiteno 14, referiteno 22 oppure si ha l'esito seguitero 13. Non sono allineate al dettato bembiano nemmeno le forme del congiuntivo presente, per lo più analogiche sulla prima coniugazione: possi 'che lei possa' 1, vadino 2, faci 'che lei faccia' 3, habbino 3, vedi 6 'che lei veda', che 'l mi vogli 6 , sappi 9 , dichi 10 , facino 12 , vengi /vengi/ 13, debbi 'che lui debba' 28; e dell'imperfetto, con la consueta variabilità: tenissero 8 , dovesseno 10, fossamo 16 per la I plurale, ecc. Al condizionale dominano le forme in -ìa (faria 4, voria 6 , manderia 8 , saperia 9 , doveria 11 , haveria 12 , veniria 18, ecc.), senza eccezioni in questo corpus. Per il futuro, tra molte forme non sincopate - sul tipo di vederà 4, saperà 29 o venirano 30 -, sono notevoli andarano 23, mantenirano 23, ecc. Per i participi, meritano una segnalazione le forme ridotte intercette 12, conza (e non conza') 38, monstro 42.

\subsection{Lessico}

Dalle lettere emergono alcuni termini pertinenti al lessico tecnico della marineria. Ho individuato almeno schirazzi 2 'piccole navi da carico usate da veneziani e levantini' (probabilmente voce turca) e galie bastarde 10 'galee capienti, con più ordini di banchi rispetto alle galee comuni', dette anche sotil. Compaiono una galeota 4, cioè una 'piccola galea monoalbero', e una galia capitana 13, che è invece la nave ammiraglia. Gli scapoli 7 sono i marinai della galea. A 8 è citato un bergantin, cioè un 'brigantino', un piccolo veliero a due alberi simile alla galea. Piccole imbarcazioni sono anche la fregata 11 e le fuste 13. I sopracomiti 16 erano i capitani delle imbarcazioni.

Altro settore ovviamente molto testimoniato nelle lettere è quello merceologico. Per esempio, compaiono le carisee 2 'abiti di lana fabbricati a Kersey, Inghilterra'. In ambito economico trovo anche ducati 50 di camera 1 , cioè 'di corso legale', con riferimento alla camera fiscale, vale a dire l'ufficio della Serenissima in cui era custodito il tesoro pubblico. Le monete erano i pizoli 1, i ducati e gli scudi. L'operatore del banco era il fattor 4 .

Sono inoltre frequenti, per via degli argomenti trattati, forme lessicali che riguardano il settore militare, come la collocazione guerra gagliarda 10 , che si potrebbe glossare come 'conflitto con grande spiegamento di mezzi' (ma la parola piace a Ramusio, che a 23 scrive: «se mantenirano gagliardamente. Altro non li ho a dire. Qui si pensa di far gagliardissime 
provision de danari»), vaivoda 'condottiero dell'esercito ottomano' 12 (slavismo), ballota 'palla di cannone' 13.

Termini tecnici sono anche advocato fiscal 'avvocato concistoriale' 11, proveditor 'magistrato' 14, bailo 'ambasciatore presso la Porta Ottomana' 14, o ancora banda 'lamierino' 30, etichettato TS anche nel GRADIT. Come unità di misura era ancora usata la pertica: 100 pertighe misurerebbe la futura fortificazione di Corfù, vale a dire poco più di 200 metri, secondo la corrispondenza media per i territori orientali d'Italia (la pertica era una misura molto variabile).

Tra gli esotismi, numerosi, conto gianizari 'guardie del sultano' 7, bassà 'pascià' 8, vaivoda 12, usbei 16 (sospetto un passaggio dal nome proprio Özbek, condottiero turco, al nome comune per indicare una carica militare), sanzaco 'sangiacco, governatore di circoscrizione ottomana' 17, zaus 31 (di etimologia incerta, dovrebbe indicare un 'messo') e tra i germanismi l'etnico Sguizari 'Svizzeri' 1 e lanzenechi 23.

Tra le parole locali, meritano una segnalazione bertizzava 'scherzavo' 10, mitria 'mitra' 11, fontego 'fondaco' 14, giesia 'chiesa' 29, e inoltre le fraseologie li tocharò la mano 8 , che significa 'concluderò un contratto, stringerò un accordo'; batuta a' fondi 9, cioè 'colpita fino ad affondare'; non la voglio più per conto alcuno 'non la voglio più per nessun motivo' 10, a furia 'in gran fretta' 11, mi è forzo da accompagnarmi 'sono obbligato a trovare compagnia' 33 , imboscati come polesini 'nascosti come pulcini' 38 , tutte naturalmente usate con intenzionalità espressiva e adatte a un registro colloquiale.

Tracce della lingua amministrativa rimangono anche nell'uso del latino, che qua e là affiora nelle lettere: item 6 , tamen 9 , re vera 10 , aliter 13 , et cetera 15, tandem 16, solum 18, laborabat in extremis 19, tandem 20 , quondam 23, immediate 28, maria et montes 43.

\subsection{Sintassi e testualità}

La sintassi informale delle lettere offre diversi spunti di riflessione, iniziando dall'uso dei modi e dei tempi verbali. Il condizionale passato può essere sostituito dall'imperfetto: «Hozi non li scrivea se non che sono gionte lettere del locotenente de Friul» 12. Il futuro nel passato, solitamente espresso con il condizionale presente (e non passato, come nell'italiano contemporaneo), o meno spesso con l'imperfetto, può essere espresso anche con il futuro se si ritiene che l'azione non si sia ancora compiuta: «stavan tutti i Francesi de mala voglia et non sapevan come tornaran indietro» 29. Il racconto degli eventi, nelle lettere, può procedere sia al presente, sia al passato; la mancata revisione dei testi, scritti in fretta, può lasciare traccia di alcune variazioni, come nel caso seguente: 
Il signore principe Doria ha voluto al tutto passar fino a Genoa, come per lettera de 22 da Roma se ha inteso, per fornirse de infinite cose che li mancavan. Et le sue galie erano molto mal conditionate, et subito messosi ad ordine ritornava in Levante. (lett. 27 del 24 settembre 1537)

Si aggiunge un caso di concordanza che sembra impermeabile ai generi: «se questi dì passati vi fosse stato cosa di nove ge la haveria scritto» 12 . Il primo participio (stato) non anticipa il genere femminile della parola che segue ( $\cos a)$, recuperato però dall'anaforico (la), ma non dal secondo participio (scritto). Un altro caso mostra l'affioramento di un tipico fenomeno substandard, la concordanza ad sensum: «l'armata turchescha, nel ditto giorno, a bon hora si levò tutta con tutte le genti et cavalli, et andavan verso Levante» 15, in cui la frase coordinata mostra un verbo al plurale a fronte di un soggetto collettivo al singolare.

Rilevanti sono anche questioni di coesione, come l'espressione del tema. Si vedano gli esempi: «et la galeota [Ø] armò de alcuni Turchi che 'l prese» 4 e «vedendo che i hanno perso quella sua prima furia et che già sono 27 dì che [Ø] sono in Italia» 40, nei quali il tema non è sempre espresso, ma può essere richiamato mediante il pronome soggetto atono, che svolge funzione coesiva senza appesantire la naturalezza del discorso.

Molto interessante è anche la scelta di alcuni deittici; Ramusio non impiega codesto e altri riferimenti di provenienza toscana (costì, costà, ecc.), ma adotta topodeittici legati all'origo veneziana, con almeno una eccezione: «Mi ha ditto messer Tomaso Gionta come è venuto a casa de vostra signoria qui in Padoa un secretario» 45, nella quale il qui sarà riferibile piuttosto al destinatario Bembo nel momento in cui legge la missiva (che è firmata da Venezia). Nel corpus compare anche un curioso caso di deittico discreto: «questa che sarà qui inclusa, per importarmi grandemente, quella sia contenta di farla mandar alla villa con ordinar che la mandino al mio fattor» 2, con cui Ramusio si riferisce dapprima alla lettera come oggetto vicino a chi la riceve, ma poi lontano da chi l'ha inviata. I logodeittici sono frequentissimi: la ditta armata 1, la ditta maestà 1, li prefati dui capitani 5, le sopradette lettere 19, ecc. Nella lettera 14 ne trovo addirittura cinque: il ditto capitano, ditto proveditor, lettera de 11 del ditto, al ditto porto, ditto principe 14. In alcuni casi possono assumere la forma di anadeittico, come in «Barbarossa havendo inteso questa cosa era venuto a trovar il principe» 7, o «era andato dapoi questo fatto verso la Boca de Cataro» 8, ecc. Talora il logodeittico svolge anche la funzione di incapsulatore cataforico: «Vostra signoria sia contenta de farmi questo piacere: de far venir a lei il mio fattor et intender da lui per che causa el non mi manda certi danari che li ho scritto 20 volte» 8 , o in un racconto ben più lungo:

Il caso de messer Philippo Stroçi et di messer Baccio Valori è in questo modo: che havendo costoro, con il mezo del re de Franza, fatto da 5 in 
6000 fanti et andando verso Prato a Montemurlo, questi dui capitani, tolti da 1000 fanti, caminorono per molte miglia discosti dal resto degli fanti ad un loco del Valori, detto la Fabrica, dove allogiatisi non temendo cosa alcuna, come fu su l'alba il signore Alexandro Vitelli, che sapeva la sua venuta, si era partito di Fiorenza con tutti li Spagnoli et cavalli che 'l havea, che non passavano da 1200 in tutto, gli fu adosso et ruppe, et preso li prefati dui capitani, cioè Strozi et Valori, et mandatili pregioni a Fiorenza, andò a trovar il resto di fanti, quali se dice haver rotto, et che era morto il figliuol de messer Philippo nomenato Pietro. (lett. 5 del 3 agosto 1537)

Come è tipico dei testi scritti poco sorvegliati, affiorano casi di dislocazione del tema, con elementi di ripresa:

La inclusa vostra signoria mi perdoni et la prego vedi di farla mandar al mio fattor, qual penso che sia amalato, non mi respondendo già un mese a 25 lettere che li ho scritto. (lett. 6 del 4 agosto 1537)

Esempio che si cita in contesto allargato per mostrare l'uso del gerundio causale che alleggerisce la subordinazione. Ancora: «L'incluse vostra signoria sarà contenta farle dar al mio Carlo» 30, oppure, senza anaforico ma con semplice inversione e enfasi dell'oggetto: «Le sue genti che sono andate verso Fermo ha scritto che subito se ne vadino de longo verso Brandizo» 24. Un esempio di frase scissa: «et è stata de notte che Todeschi dormendo sono sta' assaltati» 36 . Compare anche almeno un caso di frase pseudoscissa: «et la causa è questa che non ghe li mando hozi, perché mi bisogna andar in Pregadi» 4. Casi di inversione, con sfumatura tematizzante, sono «et pagato che haverò» 3 , «fatto che io hebbi far li conti» 4 , ecc.

Malgrado la frammentazione degli argomenti nelle lettere, può accadere che Ramusio indulga in sequenze di proposizioni subordinate. Un caso abnorme è il seguente:

Hozi vi son lettere da Roma de 21 che mandano lettere da un nostro viceconsolo da Otranto de 15, come l'armata si era levata come fu ditto alli 12, havendo brusato Ugento et Castro et li lochi circumvicini, che sopra ditta armata, per molti schiavi christiani scampati se intendeva esservi l'orator del re cristianissimo et che l'usbei capitano di quella, havendo aspettato Barbarossa 21 giorno [sic], qual li havea promesso in termine di 4 giorni venirlo a trovare, dubitando che 'l non fosse andato in Barbaria over non havesse fatto qualche tradimento, si era levato et andava verso la Vallona. (lett. 16 del 24 agosto 1537)

Il pronome relativo quale può essere espresso anche senza articolo: «tolse, ancora, de una nave nostra venitiana, quale era andata in Ancona», 
ecc. 2, «io ho rapresentato la lettera de cambio al Sassetto [...] qual mi ha promesso di darmeli domattina al tutto» 3, «andò a trovar il resto di fanti, quali se dice haver rotto» 5. Nella forma che esso può essere omesso, come avviene di frequente ancora a questa altezza cronologica malgrado le indicazioni bembiane, improntate alla razionalità anche sintattica. Se ne vedano alcuni esempi: «altro non dirò salvo [Ø] a vostra signoria mi raccomando» 1 , passim (ma nella formula finale si notano molte oscillazioni), «l'orator francese e Troilo Pignatello sono gionti a Castro con summa assai de danari per far fanti da mantenir li lochi [Ø] si prenderano a nome di Franza» 11, ecc. Al contrario, in un caso il che viene ripreso dopo incidentale: «dubitano li rettori che, essendo vera la rotta data per Turchi al capitano de l'imperator detto Cocianer, che tutte quelle genti venivano a' danni da quelli contadi» 35. Il secondo che è stato peraltro aggiunto in interlinea, per rimarcare i rapporti sintattici. Anche la preposizione $d i$ introduttrice di subordinata dopo verbi transitivi può essere omessa (la struttura ricalca la forma latina delle oggettive e dichiarative all'infinito); basterà un unico esempio tra i tanti: «ditto signor Hippolito dice haverlo fatto» 17. Può esserne evidenziata la mancata espressione anche come segnacaso: «hoggi farò far il conto [Ø] quel che monta il tutto» 3; qui se ha grandissimo fastidio [Ø] quel che terminerà questo sdegno del Turco conceputo contra li Venetiani che li hanno fatto tanti oltraggi»18; «heri sera a due hore di notte vi furono lettere da Roma che mandano lettere del viceré da Otranto de 17 [Ø] come in quel porto era gionta una fregata» 28.

Merita una trattazione a parte l'illustrazione degli usi del che subordinatore generico, o 'polivalente'. Il valore più diffuso è quello causale, come nei casi seguenti: «pertanto vostra signoria faci summar li danari che io ho speso in questo pagamento, che di tanto io ho fatto haver receputo dal ditto commesso del signor Zuan Ioachin» 4; «non fu vero di quelle 5 galee che li nostri de Corfù, per le ultime sue 4 de l'instante, ne lo haverian scritto» 26. Con valore consecutivo: «non vi è ordine che 'l mi vogli respondere, che mi despiero» 6. Con valore temporale: «contra li qual mi par che si habbin portato male Spagnuoli ad andarvi di notte et piovendo che fu il primo dì di agosto» 5. Ma non mancano casi, che oggi definiremmo substandard, di uso del che in sostituzione delle forme relative oblique: «et un altro fiorentino che mi ho domenticato il nome» 17 , o «si pensa che forsi l'haverà ancho Turrino per causa delle vittuarie che ne han mancamento» 30. Interessante è anche l'impiego del che dichiarativo, piuttosto utile per riportare le notizie: «le lettere da Roma dicono che 'l marchese de Agilar [...] ha una particolarità de più, che 'l ditto nostro capitano era andato dapoi questo fatto verso la Boca de Cataro per congiongersi con l'altro general nostro Vitturi...» 8. Non mi soffermo in queste analisi sul discorso riportato, che segue sempre le modalità del discorso indiretto e fa ampio uso di verba dicendi, non sempre con esigenze di variatio. 


\section{Riepilogo}

Rispetto al corpus di testi analizzato in Romanini (2007, 255-84) emergono evidenti differenze, non imputabili unicamente a motivazioni diacroniche, cioè a una possibile evoluzione toscaneggiante dell'idioletto di Ramusio (pure possibile nell'arco considerato, che si estende dalla fine degli anni Trenta, per le lettere a Bembo qui considerate, alla fine degli anni Quaranta, per i testi pubblicati nel primo libro delle Navigationi). Piuttosto, la forte caratterizzazione locale delle epistole sarà da imputare alla destinazione privata dei testi stessi, che consente una maggiore velocità di scrittura e un tempo minimo di progettazione. Le missive inviate da Ramusio a Bembo, inoltre, hanno cadenza serrata, e ciò induce a una ulteriore diminuzione del registro e delle convenzioni testuali, poiché il canale di comunicazione resta di fatto sempre aperto. Benché il destinatario delle lettere sia Pietro Bembo, l'autore delle Prose della volgar lingua, Ramusio non avverte per questi testi l'esigenza di fare sfoggio della sua capacità di scrivere toscaneggiando, e indulge volentieri in localismi, in particolare quando l'espressività lo richieda. L'analisi della sintassi mostra peraltro che, oltre a coloriture fonetiche, morfologiche e lessicali, la lingua presenta anche una progettualità modesta, coerente con le indicazioni fornite dallo spoglio delle altre strutture. 


\title{
«Per convenienti rispetti» Osservazioni sulla presa di parola di G.B. Ramusio
}

\author{
Toni Veneri \\ (University of North Carolina at Chapel Hill, USA)
}

\begin{abstract}
This article delves into the rhetorical fabric of the discorsi the Venetian humanist Giovanni Battista Ramusio crafted to introduce his collection of travel accounts (Navigazioni e viaggi, 1550-9), with the aim of unearthing some of their deliberately unspoken contents. An analysis of Ramusio's reticence regarding certain matters, together with a set of broken allusions, and his highly controlled self-presentation, allows a reframing of his locus of enunciation in the light of his self-fashioning within the Venetian social and intellectual environment, his ideological objectives and strategic constraints, and the epistemic stances implied in his editorial choices.
\end{abstract}

Sommario 1 L'archivio' ramusiano. - 2 Reticenza autoriale: la modestia di Ramusio. - 3 Reticenze ideologiche e strategiche. - 4 Reticenze epistemiche. -5 Le curvature biocentriche del discorso ramusiano.

Keywords Giovanni Battista Ramusio. Renaissance travel literature. Renaissance self-fashioning. Venice. Cartography.

\section{$1 \quad$ L'archivio' ramusiano}

I contorni della figura professionale ed editoriale di Giovanni Battista Ramusio, così come quelli della sua persona materiale, rimangono per molti versi sfuggenti. Le informazioni disponibili rimandano, com'è lecito aspettarsi, a un insieme di materiali formalizzati - libri, contratti, lettere, mappe, medaglie, iscrizioni - che sono sopravvissuti al passare del tempo e che direttamente o indirettamente recano traccia della sua esistenza storica. Se un vero e proprio archivio ramusiano non esiste, nel senso tecnico di fondo (o di luogo) deliberatamente costituito per accoglierne i documenti prodotti o acquisiti in vita, è attraverso la raccolta e la comparazione di tali materiali disparati che gli studiosi, non diversamente che per altri personaggi storici, ne hanno di volta in volta ricostruito il profilo biografico. E dunque ricorrendo a un'accezione più libera e circostanziale del termine che propongo di chiamare, nelle pagine che seguono, questo insieme il nucleo primario dell' 'archivio' ramusiano. È attribuendo significato a questi documenti, definendoli tali, inferendone rapporti di causalità ed eventualmente di figuralità (laddove 
un dato verrebbe ad anticipare o prefigurare un altro storicamente successivo, cf. White 1999, 95) che, secondo un moderno abito storiografico occidentale, è stato ed è ancora possibile inserirne la figura all'interno di sempre nuove narrazioni - secondo un procedimento che Paul Veyne ha definito «construction d'intrigues» $(1971,46)$ e Hayden White di «emplotment» (1973, 5-11).

Più ancora che dalla ricombinazione dei pezzi o dal ritrovamento di nuovi elementi documentari, questa riscrittura storiografica sembra nutrirsi dell'interazione con altri e più vasti 'archivi' della modernità, primi fra tutti quelli disciplinari. In questo senso, le varie narrazioni storiche che forniscono l'ossatura diegetica ad altrettante tradizioni disciplinari - la storia della scienza, della geografia, della letteratura, dei viaggi, di Venezia, e così via - selezionando e organizzando i dati e le informazioni loro congeniali, hanno illuminato la figura di Ramusio di volta in volta di una luce differente, conferendogli un ruolo e un risalto di volta in volta diversi. Così per Antonio Del Piero, autore di una monografia pubblicata nel 1902, l'importanza di Ramusio sta nell'aver iniziato il metodo positivo e sperimentale in geografia, di contro alla tendenza alla declamazione retorica del tempo. ${ }^{1}$ Similmente per Stefano Grande, rispetto ai predecessori e ai contemporanei, «la figura del Ramusio si solleva [e] al raccoglitore sottentra il vero scienziato» $(1905,108)$ capace, in collaborazione con Girolamo Fracastoro e Giacomo Gastaldi, di portare un triplice assalto all'autorità di Tolomeo in campo astronomico, geografico e cartografico (100-1). Ridimensionata appare invece la figura di Ramusio negli studi di George B. Parks, che pure a metà del secolo scorso, individuando le fonti delle Navigazioni $e$ viaggi, ha contribuito in maniera decisiva all'incremento dell' 'archivio' ramusiano (1955a, 1955b). Rileggendo la raccolta quale articolata operazione di riciclo dei prodotti dell'editoria veneziana, rimarcando con stupore, in tale operazione, l'estraneità di attività editoriale e attività cancelleresca, intendendo gli interessi del segretario in termini puramente libreschi, paragonando infine quest'ultimo alla spericolata figura di imprenditore coloniale di Richard Hakluyt, Parks riconosce nell'opera di Ramusio, «spettatore della storia» (1955b, 129), uno scollamento fra

1 «Il Pomponazzi inizia il metodo positivo nella filosofia [...], il Ramusio nella Geografia s'affida al metodo esclusivamente sperimentale, contro la generale prevalenza del retoricume classico» (Del Piero 1902, 24); «l'uomo il quale più che alle tradizioni, sieno anche classiche, presta fede ai fatti ch'ei vede e osserva; metodo di studio che offre le più sicure garanzie per un geografo, il quale non su tradizioni, ma sui fatti fisici e antropologici può solo fondare le sue induzioni e deduzioni [...] il metodo geografico del Ramusio è nettamente distinto da quello della generalità de' suoi contemporanei» (36-7); «insistiamo di proposito sopra la questione del metodo, perché nelle discipline positive che non posson andar disgiunte dall'esperienza, il metodo è l'origine, il principio e la base di progresso vero; senz'esso avremo della declamazione retorica, potremo avere anche dell'arte in campo usurpato, ma della scienza mai» (89). 
realtà (storia) e rappresentazione (letteratura) che in ultima analisi ne sminuisce drasticamente il valore. ${ }^{2}$

L'edizione moderna delle Navigazioni, curata da Marica Milanesi (19781988), è stata invece determinante nel rivalutare il ruolo di Ramusio all'interno della storia europea delle conoscenze geografiche, quanto nell'attirare l'attenzione sul valore documentario dei testi pubblicati nella raccolta (Milanesi 1978, 1984, 1994). Diverse analisi storico-filologiche rivolte a singoli testi - il Milione innanzitutto ${ }^{3}$ - hanno così recentemente contribuito a ripensare la figura erudita di Ramusio in termini editoriali, insistendo sulla sua cruciale responsabilità nella trasmissione di un vasto corpus di letteratura di viaggio. Con le continue modificazioni e ibridazioni degli assetti disciplinari in corso, l'archivio promette di estendersi ulteriormente. In particolare, lo spatial turn delle discipline umanistiche permetterebbe di rileggere le Navigazioni nei termini epistemologici dell'emergenza e dell'istituzione di un nuovo discorso sullo spazio, che si fa strada in un momento storico in cui in Europa si affrontano e confliggono paradigmi diversi. ${ }^{4}$ Allo stesso tempo l'opzione decoloniale permetterebbe di riconoscere alcune profonde implicazioni inerenti all'oggettivazione universalista del planisfero ramusiano, laddove la produzione europea di conoscenze geografiche diventa nel Rinascimento anche produzione di 'differenza coloniale', e la svalutazione o la rimozione di culture e pensieri non europei viene ad articolarsi all'interno di un contesto storico e geografico di cui la colonizzazione è ormai parte integrante. ${ }^{\mathbf{5}}$

2 «Otherwise his work suggests that he was a spectator of history. It is probably more important that as a Venetian he could not escape the influence of Venetian printers, who led the way in publishing and republishing the travel narratives of the age of discovery. Indeed, it may appear that the Navigationi is not so much the climax of Ramusio's own work as it is of the decades of labor of Venetian publishers. Altogether it is striking that Ramusio had no vested interest in travel itself. His follower Hakluyt in England was from the start a promoter of English enterprise overseas, and naturally became its historian as well. Ramusio, so far as we can tell, was or became such a historian because of a purely bookish interest. [...] It is further surprising that Ramusio's occupation seems to have had no direct influence on the Navigationi. At the age of twenty he entered on his lifelong service to the state as a clerk in the Chancellery [...]. In these fifty years of service to the state, he naturally had access to many men and many documents, but I cannot find that he drew seriously upon either for his travel collections. He published no Venetian documents at all, no reports of Venetian envoys which might have contained important news from Lisbon or Seville on overseas affairs. And his relations with other diplomats were in most cases personal rather than official. It is true that Ramusio's own official duties promised well for one who was to be interested in travel literature» (Parks 1955b, 129).

3 Mi limito qui a segnalare il volume Burgio 2011 e l'edizione critica digitale, presentata in forma ipertestuale, Simion, Burgio 2015.

4 Fra i molti testi che suggeriscono questa linea di analisi, con particolare attenzione al periodo rinascimentale, mi limito qui a segnalare de Certeau 1990; Lefebvre 2000; Farinelli 2003; Short 2004.

5 A riguardo cf. Mignolo 1995, 219-313 e Mignolo 2011. 
Che l'archivio dovesse includere tanto le fonti primarie quanto quelle secondarie (ascrivibili al regime del commento, dell'interpretazione, della storiografia) era già ben chiaro a Emanuele Cicogna che per Ramusio, così come per innumerevoli altre figure storiche veneziane, ha lasciato, sotto forma di lista commentata, un ricchissimo inventario cui, all'epoca, si sarebbe probabilmente potuto aggiungere ben poco (1824-53, 2: 31530). Il profilo biografico inserito nelle Inscrizioni veneziane, che integra le informazioni trasmesse da una cronaca familiare seicentesca con riferimenti contenuti in opere contemporanee e successive, rimane dunque, assieme agli importanti e più recenti ritrovamenti di Massimo Donattini (1980 e 2007), l'insuperato punto di partenza per ogni studioso di Ramusio. Un archivio, dunque, costitutivamente incompleto e necessariamente aperto, tanto all'integrazione di fonti primarie, quanto alla stratificazione di letture secondarie; un archivio autoreferenziale, nella misura in cui i suoi elementi acquistano significato in quanto testimonianze del soggetto o dell'evento istituito dalla fondazione stessa dell'archivio; un archivio allo stesso tempo fondamentalmente opaco, nella misura in cui rigetta dietro le proprie quinte le circostanze della propria formazione.

Occasionalmente però è l'archivio stesso ad attirare l'attenzione sulle condizioni precarie della propria costituzione e sulle circostanze contingenti che ne possono determinare la dissoluzione o la distruzione. È così che nelle stesse Navigazioni si apprende dall'editore Tommaso Giunti la gravità del danno provocato dall'incendio che il 4 novembre 1557 distrusse la stamperia di san Giuliano, portando con sé i materiali preparatori per l'edizione del secondo e forse anche del quarto volume, mai pubblicato, della raccolta (Milanesi 1978-88, 4: 3). È così che anche in Venetia città nobilissima Francesco Sansovino elenca con rammarico i nomi delle personalità veneziane, o legate a Venezia, i cui ritratti 'di gruppo' figuravano nella sala del Maggior Consiglio prima che un altro incendio, nel 1577, danneggiasse irreparabilmente diversi ambienti di Palazzo Ducale. In questo pantheon di uomini illustri, ricorda Sansovino, figurava il giurista Paolo Ramusio il vecchio, padre di Giovanni Battista, accanto a Gentile Bellini, mentre all'interno di una tela di Paolo Veronese

sopra un poggiolo erano ritratti Andrea Gradenigo padre di Luigi con veste senatoria, e Giovanni Battista Ramusio secretario del Consiglio dei Dieci, che fu padre di Paolo, i quali pareva che ragionassero insieme [...]. Le quali tutte cose consumate dal fuoco del 1577 apportarono gran dispiacere a tutto l'universale, per la perdita delle fatture di tanti valenti huomini, e delle memorie di tanti personaggi eccellenti, de quali il mondo è rare volte copioso. (Sansovino 1581, 132-3)

Più di un secolo dopo, Otto Mencke, nella sua biografia di Fracastoro, attribuiva il profondo silenzio degli eruditi attorno alla figura di Ramusio, 
fra le altre cose, alla mancanza di monumenti pubblici che ne celebrassero l'ingegno. ${ }^{6}$ La fisionomia del segretario non è stata però del tutto consegnata alla fantasia degli artisti moderni: una medaglia cinquecentesca di bronzo fuso, attribuita a Giulio della Torre, proveniente dalla collezione di Giovanni Battista Mazzucchelli e da lì confluita negli inventari dei Musei Civici di Brescia, restituisce sul dritto l'effigie di Ramusio e sul verso un globo terracqueo con il Nuovo Mondo (Voltolina 1998, 256). Il ritratto che è però servito da modello per alcune incisioni ottocentesche e primonovecentesche rimane quello dipinto nel 1762 da Giustino Menescardi - «parto piuttosto della fantasia del pittore, che della verità» secondo Cicogna (1824-53, 2: 322) - a ornamento di una grande tavola del Mediterraneo, esposta nella Sala dello Scudo di Palazzo Ducale. Quest'ultima, va notato, si pone al centro del rifacimento, coordinato da Francesco Griselini, di un ciclo cartografico originariamente concepito per quell'ambiente di ricevimento dallo stesso Ramusio e da Giacomo Gastaldi (cf. Gallo 1943).

A naufragi documentari dovuti a circostanze contingenti e fattori 'esogeni' è possibile però attribuire solo in parte la penuria di notizie sulla vita e l'opera di Ramusio, il quale in vita sembra anzi aver deliberatamente sorvegliato e contenuto la circolazione di informazioni sul proprio conto. Rispetto alle tracce lasciate dalle figure cui Ramusio viene tradizionalmente associato - Pietro Bembo, Girolamo Fracastoro, Andrea Navagero - quanto di lui ci è invece pervenuto, a partire dalla grande silloge delle Navigazioni $e$ viaggi e dai volumi e documenti variamente collegati alla sua redazione, appare caratterizzato fin dall'origine da un certo grado di reticenza: una studiata cautela nell'espressione, un trattenere tacendo, che sarebbe fuorviante ricondurre unicamente alle regole e ai codici retorici dei vari generi discorsivi da lui praticati e consegnati all'archivio (epistolare, espositivo, burocratico, narrativo).

\section{Reticenza autoriale: la modestia di Ramusio}

Una prima macroscopica reticenza riguarda la visibilità stessa di Ramusio e la pubblicità del suo nome, in altre parole la mancata assunzione di autorialità o responsabilità editoriale nelle opere da lui allestite. Le ricerche d'archivio condotte da Massimo Donattini (1980, 73-5; 1992, 124-9) hanno dimostrato il suo coinvolgimento, a lungo ignorato, nella curatela e nella traduzione di due fra le maggiori pubblicazioni di argomento americano uscite a Venezia nella prima metà del secolo, il Summario de le Indie Occidentali (Anghiera, Oviedo y Valdés 1534) e il Viaggio fatto dagli Spa-

6 «Magis hic negotiis publicis et doctrina civibus suis, quam scriptis posteritati innotuit. Nulla, quantum ego scio publica ingenii ejus monumenta extant. Quae causa est, ni fallor, cur penes vulgares vitarum scriptores altum sit de Rhamnusio silentium» (Mencke 1731, 142). 
gniuoli a torno a'l mondo (Transilvano, Pigafetta 1536). In parte diverso, ma altrettanto eloquente, è il caso delle Navigazioni, i cui due volumi pubblicati Ramusio vivente - il primo e il terzo, rispettivamente nel 1550 e nel 1556 - mantengono l'anonimato del curatore, ma in termini puramente fittizi. Gli indizi di tale finzione sono numerosi, a partire dalla dedica a Fracastoro che apre il primo volume e che, attraverso una studiata tessitura retorica, si muove con sapienza fra i poli asimmetrici del patrono (Vostra Eccellenza) e dell'io petitore, alternando come d'uso i toni encomiastici della captatio benevolentiae a manierati understatement e preterizioni:

Nella qual opera quanto un debile e piccolo ingegno come è il mio abbia durato di fatica, massimamente per la diversità delle lingue nelle quali detti auttori hanno scritto, non voglio ora dirlo, accioché non para che con parole aggrandisca le fatiche e vigilie mie: ma i benigni lettori, a ciò pensando, spero che per se medesimi in qualche parte lo conosceranno. E se pur noi abbiamo mancato in molti luoghi - il che confesso esser il vero -, non è però proceduto dalla poca diligenza nostra, ma più tosto perché il valor dell'ingegno non ha potuto pareggiarsi all'ardore della buona volontà. (Milanesi 1978-88, 1: 5)

L'effetto ricercato è quello inverso di delineare enfaticamente l'investimento personale del raccoglitore e la portata scientifica dell'impresa editoriale, validata e rafforzata piuttosto che ridimensionata dal patrocinio di Fracastoro. Ma c'è di più: sottolineando il legame affettivo («ho voluto lasciare a' nostri posteri con questa mia fatica quasi una testimonianza della nostra lunga e santa amicizia») e il debito intellettuale nei confronti del dedicatario («fu quella che da principio m'indusse con la sua auttorità a questa impresa»), un debito inoltre coltivato attraverso i «savi discorsi e dolci ragionamenti avuti col magnifico conte Rimondo dalla Torre», il raccoglitore pone la sua anonima ma già ingombrante figura all'interno di un contesto sociale ben definito e capace di conferire ulteriore autorevolezza all'opera (Milanesi 1978-88, 1: 3). E se tale contesto riemerge a più riprese nei Discorsi che introducono i vari racconti di viaggio, nello stesso volume del 1550, a chiarire gli eventuali dubbi di un lettore non familiare con la nomenclatura dei circoli intellettuali veneziani, viene in aiuto la risposta di Fracastoro al Discorso sopra il crescer del fiume Nilo (ancora 'anonimo' in questa edizione), con l'esplicita indicazione che il destinatario del trattato non è altri che il segretario messer Giovanni Battista Ramusio. La finzione si palesa ulteriormente nel momento della sua rottura: nella nota indirizzata ai lettori nove anni più tardi in apertura al secondo volume (1559), Giunti indica la morte di Ramusio fra le concause del ritardo della pubblicazione prima ancora di adempiere all'obbligo formale di rivelarne al pubblico l'identità di curatore delle Navigazioni - e di celebrarne conseguentemente la memoria. Assieme al commosso ricordo 
dell'amico e collaboratore, l'editore spiega in termini etico-psicologici la scelta deliberata di Ramusio di non associare la raccolta al suo nome:

Le qual sue fatiche giudiciose e onorevoli, se non usciron fuori illustrate col suo nome, avvenne per la sua singular modestia, che in ciascuna sua azione continuamente era solito d'usare, di modo che vivendo, non comportò mai che vi fusse posto, come uomo ch'era lontano da ogni ambizione e aveva l'animo indirizzato solamente a giovare altrui. Ma io, che mentre egli visse, l'amai infinitamente sopra ciascun altro, e morto l'amerò infin che durerà la vita mia, sì come ho desiderato, così anche son tenuto a far tutte quelle cose, le quali io stimi che siano per acquistargli alcuna fama: non posso e non debbo in queste sue utili e onorate fatiche ormai tener più celato il nome suo, del quale ora vedrete ornato questo secondo, che pur finalmente mandiamo in luce. (Milanesi 1978-88, 4: 4)

Il nome - in questo caso, probabilmente per ragioni di allestimento tipografico - comparirà però sul frontespizio solamente nella seconda edizione del primo volume (1563), seguito da una nota giuntina che riprende e sviluppa il passo sopra citato. Il commento, che a sua volta si nutre di drammatizzazioni retoriche, conferma la volontà di Ramusio di mantenere un profilo arretrato. Tuttavia, così come le istanze ideologiche, politiche ed economiche che attraversano la raccolta ne smentiscono il carattere disinteressato - su cui invece compilatore ed editore insistono nelle loro dichiarazioni - così anche la scelta di Ramusio di non comparire in prima fila, qualora ricondotta alla sua posizione sociale e cancelleresca, sembra eccedere i tratti di un'indole psicologica individuale («la sua singular modestia») e al contrario inserirsi in una traiettoria strategicamente poco visibile di ascesa e promozione sociale.

Da questo punto di vista, è utile ricordare la particolare posizione di Ramusio, che riveste le sue funzioni di segretario anche in ragione della sua appartenenza al ceto dei cittadini originari - fascia intermedia compressa fra nobiltà e popolo, all'interno di una costituzione che non fa che ribadire tale dicotomia. Sono gli anni in cui nella «città della calma e del silenzio politico» - così le parole di Jacob Burckhardt $(1955,61)$ - dove le classi sociali sembrano non avere interessi contrastanti, l'ideale umanistico dello stato misto viene consegnato al mito veneziano, non soltanto per proiettare prestigio politico e compiacere l'orgoglio municipale, ma anche per distogliere l'attenzione dallo strapotere che si sta accumulando nell'oligarchia del Collegio. Andrea Navagero, recitando l'elogio del defunto doge Leonardo Loredan, suffraga l'eccellenza dell'aristocrazia sulle altre forme di governo; Marc'Antonio Sabellico esalta nella Repubblica l'incarnazione dell'ideale platonico di repubblica aristocratica; il cardinale Gasparo Contarini riesce a imperniare sullo stato misto la sublimazione 
ideologica, entro una cornice aristocratica, della tendenza all'oligarchia (cf. Ventura 1980). Lo sforzo di autodefinizione della classe dirigente veneziana dà luogo, su un sostrato umanistico, a un'ideologia del patriziato convinta dell'eccellenza naturale della nobiltà, e che distingue recisamente due ordini di abitanti: nobiltà e popolo. Se i veri cittadini sono solo i patrizi, Contarini, nel suo De magistratibus et Republica Venetorum, all'interno della perfetta struttura organica e gerarchica dello stato veneziano riconosce tuttavia un ruolo importante e in certa misura privilegiato ai cittadini originari, nei cui ranghi vengono selezionati e formati i segretari e i funzionari della Cancelleria Ducale. ${ }^{7}$ Il funzionamento della grande macchina statale veneziana riposa infatti anche sulla permanenza delle loro cariche, a fronte dell'avvicendarsi dei patrizi nelle magistrature, sul loro accesso e conoscenza degli archivi, sulla loro costante presenza alle sedute del Senato e del Collegio, sulla fede et accorta diligenza con cui presiedono al paper management della Repubblica, in un contesto di sempre maggiore dipendenza dello stato moderno da documenti scritti (il cosiddetto archival turn, cf. Barzman 2014). A queste rigide strutture costituzionali Contarini fa corrispondere una duplice gerarchia morale ed economica, laddove le gradazioni sociali che distanziano popolo e nobiltà esprimono altrettanti livelli di onore e onestà. Inserito in questo disegno, il progresso nei diversi uffici del cursus honorum cancelleresco viene scandito da livelli sempre più elevati di virtù morale, ammirazione pubblica, ma anche retribuzione economica che, sottolinea il cardinale, permettono non solo di mantenere il patrimonio familiare, ma pure di accrescerlo.

Come ha segnalato Donattini (2007), le vicende della storia familiare ramusiana, da Paolo il vecchio a Giovanni Battista a Paolo il giovane, paiono

7 «Tutto 'l popolo è diviso in due maniere, percioché certi ne sono di più honorato genere, altri della bassa plebe come gli artisti, et gli huomini di sì fatta sorte, i quali Aristotele dice nella Politica, che questi sono in vece di servi publici. All'una, et all'altra maniera a mio giudicio è stato commodamente et giustamente havuto riguardo. [...] L'ordine de i Secretari è honestissimo, i quali sedono con tutti i magistrati. Questo ufficio si dà solamente a gli huomini della plebe, non a gentilhuomo veruno: il quale quantunque non sia illustre, è nondimeno honorato. Conciosia cosa che alla fede et accorta diligenza di quelli sono commessi i libri, et scritti publici, nei quali si contengono tutte le cose private, et publiche, et colui, che è stimato degno di quella impresa, ha meritamente nome di huomo da ben, et industrio, et havrà attribuita a sé la stima et a tutti è ordinata quella provisione, che non solamente è a bastanza a mantenere le cose domestiche, ma anche ad accrescerle. A questo tutti quegli, che sono secretarii del Senato, sono del popolo, niuno della nobiltà. Questo ordine è più honorato di quel di sopra: però quegli del popolo, che sono nati di gente più nobile, sogliono essercitar quello ufficio. [...] Elegonsi molti di questi, i quali servono al consiglio de' Dieci, et le cose che da quegli si trattano, scrivono in libro, et sono consapevoli di tutte quelle cose, che si fanno dal Collegio. Nel quale honore durano mentre che vivono, et non a vicenda, come i gentilhuomini nei loro magistrati, dei quali niuno è perpetuo da quel dei Procuratori in poi. Di questi, che sono secretarii del Senato, uno ottiene le prime parti, et è chiamato cancelliero di Vinegia, il quale è un grandissimo honore [...]. Questo, non è secreto nella Repubblica che egli non sappi» (Contarini 1544, LXVII-LXVIII). 
occupare e percorrere con determinazione questo stretto margine disponibile alla promozione sociale. Il caso del compilatore delle Navigazioni, la cui voce si è detto è perlopiù filtrata e formalizzata da un linguaggio letterario ad alto tasso di retoricità, appare così ulteriormente leggibile alla luce delle molteplici forme di modellamento 'artistico' dell'identità, che secondo Stephen Greenblatt in età rinascimentale vengono a contraddistinguere il comportamento e la visibilità pubblica delle élite borghesi e aristocratiche europee. In questo senso, il Renaissance self-fashioning come processo di auto-manipolazione va inteso come una risposta dialettica al sempre maggiore controllo esercitato dalle istituzioni sui loro soggetti e alla sempre più rigida disciplina imposta a questi ultimi. La risposta appare dialettica proprio in quanto la limitazione dell'autonomia nelle pratiche di autoformazione dà luogo a un'accresciuta consapevolezza dei mezzi e delle procedure attraverso cui si possono raggiungere «a distinctive personality, a characteristic address to the world, a consistent mode of perceiving and behaving» (Greenblatt 1993, 2). ${ }^{8}$

Il caso ramusiano appare allora esemplare di come il cittadino originario, in particolare il segretario che ha superato i maggiori traguardi della carriera cancelleresca, tenda a modellare la propria identità su quella del patriziato, nel rispetto di una precisa distinzione sociale avvertita anche in termini di visibilità pubblica. Privato di dirette responsabilità politiche, egli sceglie di competere con il nobile, piuttosto che nella mercatura, sul piano 'intellettuale', e attraverso una sorta di 'specializzazione': all'interno dei circoli eruditi che frequenta, Ramusio si conquista il ruolo di referente in materia geografica. Malgrado il riserbo proprio della funzione cancelleresca e la subalternità politica - alla cui luce è possibile rileggerne

8 «There is considerable empirical evidence that there may well have been less autonomy in self-fashioning in the sixteenth century than before, that family, state, and religious institutions impose a more rigid and far-reaching discipline upon their middle-class and aristocratic subjects. [...] This change is difficult to characterize in our usual ways because it is not only complex but resolutely dialectical. If we say that there is a new stress on the executive power of the will, we must say that there is the most sustained and relentless assault upon the will; if we say that there is a new social mobility, we must say that there is a new assertion of power by both family and state to determine all movement within the society; if we say that there is a heightened awareness of the existence of alternative modes of social, theological, and psychological organization, we must say that there is a new dedication to the imposition of control upon those modes and ultimately to the destruction of alternatives. Perhaps the simplest we can make is that in the sixteenth century there appears to be an increased self-consciousness about the fashioning of human identity as a manipulable, artful process. [...] Thus separated from the imitation of Christ self-fashioning acquires a new range of meanings: it describes the practice of parents and teachers; it is linked to manners or demeanor, particularly that of the elite; it may suggest hypocrisy or deception, an adherence to mere outward ceremony; it suggests representation of one's nature or intention in speech or actions. And with representation we return to literature, or rather we may grasp that self-fashioning derives its interest precisely from the fact that it functions without regard for a sharp distinction between literature and social life» (Greenblatt 1993, 1-3). 
l'infinita modestia - rispetto agli illustri amici, di cui è spesso il mediatore istituzionale, Ramusio attraverso l'interesse per la geografia si sottrae a una posizione gregaria e si inventa «un ruolo sempre più autonomo e specifico nella produzione culturale» (Milanesi 1978, XVI). Sceglie per sé un ambito di studio e ricerca che più degli altri riesce a risolvere la tensione fra ideale e reale, fra il pensiero speculativo italiano e il realismo spregiudicato dell'ideologia veneziana, che imponeva come irrinunciabile il nesso fra cultura e stato. Le relazioni degli ambasciatori, frutto autonomo e raffinato della scrittura politica veneziana, gli indicano in Senato un ambito di conoscenza che permette di rispettare e conservare questo nesso. Istituisce in casa sua, a livello familiare, una 'scuola di cosmografia' dove, assieme all'umanista bresciano Giovita Rapicio e a Giacomo Gastaldi, attende all'educazione del figlio Paolo e di altri giovani studenti. Bembo insiste perché Orazio, il figlio di Carlo Gualteruzzi da Fano, l'editore del Novellino, possa far parte di questo cenacolo pedagogico (Del Ben 2004, XXXVII-XXXVIII). Ramusio amministra inoltre il patrimonio librario marciano e con lui deve fare i conti tutta l'élite umanistica veneziana, con la quale egli non transige le condizioni dei prestiti. Come i testamenti ritrovati da Donattini dimostrano (1980, 91-8), il segretario attende anche all'incremento economico della sua posizione, e riesce così ad aderire al nuovo stile di vita che il patriziato va coltivando nelle ville di terraferma: costruisce una villa Ramusa che alla pari di quelle di Fracastoro, Raimondo della Torre, Daniele Barbaro, diventa il luogo privilegiato di convegni letterari e conversari filosofici e scientifici. Nelle strette maglie dell'ordinamento gerarchico della Serenissima, l'ascesa sociale di Ramusio avviene dunque anche lungo i binari di un'assidua attività relazionale con la nobiltà e di una differenziazione dei propri interessi scientifici che gli guadagna l'ammirazione di diversi strati dell'intellighenzia veneziana. Anche uno degli ultimi gradini del cursus honorum burocratico viene superato nel 1553: la nomina a segretario del Consiglio dei Dieci sembra quasi un premio e un riconoscimento della Repubblica riservato tanto a un suo fedelissimo servitore, quanto al suo affermato luminare geografico.

\section{Reticenze ideologiche e strategiche}

A questa stessa posizione, di segretario del Senato e infine del Consiglio dei Dieci, preposta anzitutto al controllo e alla segretezza dell'informazione politica, e che gli valeva il titolo di circospetto e fedelissimo, possono essere ricondotte almeno altre due serie di reticenze nelle Navigazioni, la prima legata alla scelta delle relazioni veneziane da includere nella raccolta, la seconda riguardante il silenzio sulla provenienza di alcuni testi inediti di area spagnola e portoghese. Nel primo caso, si tratta dell'istanza 'patriottica' veneziana, che costituisce forse l'elemento ideologico che più 
profondamente attraversa la raccolta, influenzandone e complicandone il disegno scientifico di descrizione e 'mappatura' verbale del mondo, che si vorrebbe condotto attraverso i resoconti più esaurienti e aggiornati a disposizione. Il filone 'veneziano' delle Navigazioni, volto ad affermare la precocità e la preminenza di Venezia nella storia delle scoperte e delle esplorazioni, si sforza di avere i più vasti orizzonti geografici possibili. La tesi viene infatti sviluppata fin dal primo volume, ponendo Venezia all'origine dell'epopea marittima portoghese, con l'inclusione dei viaggi di Alvise Da Mosto e Nicolo' di Conti, e l'insistenza sul dono, nel 1428, da parte del Senato veneto all'infante Pedro del Portogallo, di una copia del Milione di Marco Polo che, stando a Ramusio, «fu gran causa che tutti quelli serenissimi re s'infiammassero a voler scoprir l'India orientale, e sopra tutti il re don Giovanni» (Milanesi 1978-88, 2: 15). Grazie alla partecipazione e soprattutto al resoconto del vicentino Antonio Pigafetta, sotto il segno di San Marco Ramusio pone anche, nello stesso volume del 1550, la circumnavigazione magellanica del mondo; così come, nel terzo volume (1556), introduce le esplorazioni marittime francesi nell'America settentrionale come dirette conseguenze della spregiudicata intraprendenza del concittadino Sebastiano Caboto. Ma è nel secondo volume (1559), con i Viaggi di Messer Marco Polo e i numerosi avantesti che lo introducono, che si apre la celebrazione del ruolo di Venezia nell'esplorazione dell'Asia, come ha scritto Alvaro Barbieri: «un preciso disegno di rivendicazione dei fasti nazionali che lavora sottotraccia in tutto il tomo secondo della silloge», con Marco Polo come «sorta di prototipo del viaggiatore lagunare», «un blasone di gloria veneziana da esibire in posizione primaziale» (2015). Una celebrazione che prosegue con i viaggi quattrocenteschi di Giovan Maria Angiolello e di un anonimo mercante, assieme alle relazioni delle ambascerie di Ambrogio Contarini (1473-8) e Giosafat Barbaro (1473-5), che costituiscono il cosiddetto 'capitolo persiano' delle Navigazioni.

All'interno di un'opera che mira, fra le altre cose, a delineare e a costituire un canone e una storia della letteratura di viaggio in base al suo contenuto informativo, questa galleria di ambasciatori-mercanti risponde chiaramente alla volontà di riconoscere a Venezia un ruolo decisivo, e paragonabile a quello delle grandi potenze coloniali, nella produzione di sapere geografico. Il sotto-canone veneziano cui Ramusio fa qui spazio appare tuttavia un canone fondamentalmente passatista - lo stesso canone che avranno in mente, duecento anni più tardi, nel 1761, i Riformatori allo Studio di Padova quando incaricheranno Griselini di rifare, sotto l'occhio attento di Anton Maria Zanetti, i malridotti teleri della sala dello Scudo, al fine di consacrare la fama «de celebri veneziani che o per azzardosi viaggi consumati; o come scopritori di terre incognite, o pur anche illustratori di 
colte erudizioni geografiche e nautiche, hanno meritato comune credito». ${ }^{9}$ Oltre a quella di Marco Polo, i Riformatori desiderano vi venga onorata la memoria dei fratelli Zeno, di Pietro Querini, Giosafat Barbaro, Ambrogio Contarini, Alvise da Mosto, Sebastiano Caboto, del 'comito veneziano' (un nostromo protagonista di un viaggio da Alessandria d'Egitto in India pubblicato nelle Navigazioni), e dello stesso Ramusio. Proseguono i Riformatori: «con che queste tavole rappresenteranno d'ora innanzi i fasti interi di questa città, rispetto a scuoperte di terre nuove, o a singolari notizie di paesi incogniti e serviranno di nobile incitamento agli osservatori o per pascere la loro erudizione o per trarre esempi d'imitazione» (Gallo 1943, 100-5). Un canone veneziano che già si presume occupare una posizione d'onore in un più ampio canone dei viaggiatori italiani, che a sua volta verrà largamente recuperato in un contesto nazionale fra Otto e primo Novecento. Ora, la costituzione di questo canone va tuttavia considerata in rapporto al progetto generale di Ramusio di selezionare i testi con le descrizioni più attendibili, dettagliate e aggiornate delle varie parti del mondo. Da questo punto di vista, il recupero di un testo trecentesco come quello dei Viaggi di Marco Polo si giustifica, al di là del movente patriottico, con la valorizzazione di informazioni su territori che ormai ai tempi di Ramusio erano diventati di difficile accesso per gli europei, non più collegati come un tempo dalle reti viarie garantite dalla pax mongolica. Una riabilitazione scientifica, per la quale Ramusio confeziona il celebre racconto dell'incontro con il mercante persiano Chaggi Memet, destinato a confermare l'attendibilità dei dati e dei toponimi poliani (cf. Veneri 2012b). Ma oltre a questo specifico caso, ci si potrebbe interrogare sulle ragioni per cui i testi veneziani selezionati da Ramusio risalgano tutti almeno al secolo precedente, in altre parole siano tutto sommato datati.

A Venezia infatti nella prima metà del Cinquecento non solo affluivano relazioni e documenti intercettati dalle reti informative delle grandi potenze europee, ma diari, descrizioni e resoconti di ambasciatori, mercanti e viaggiatori veneziani, la cui redazione veniva incoraggiata nell'interesse di una classe politica che coincideva in larga parte con il ceto mercantile della città. Per limitarmi a un esempio relativo alla Persia, un'ampia relazione su questo paese, disponibile ora nell'edizione moderna di Giorgio Cardona (Membré 1969), era stata stilata per le autorità veneziane nel 1542 da quello stesso Michele Membré che figura accanto a Ramusio e a Michele Sanmicheli nell'aneddoto sopra ricordato dell'intervista a Chaggi Memet. Per spiegare dunque le selezioni ramusiane, in particolare nel capitolo asiatico delle Navigazioni, potrebbe essere insufficiente riconoscere, con Barbieri, che

9 Il documento è stato pubblicato in Gallo 1943, 100-5. 
riaffermare il contributo della Serenissima all'esplorazione dei quadranti orientali serviva verisimilmente a compensare l'amarezza di un presente assai meno brillante e la presa d'atto di un ridimensionamento, ovvero di un orizzonte reso ormai angusto dal confronto con i nuovi e sconfinati scenari rivelati dalle imprese marittime promosse dalle corone di Portogallo e di Spagna. (2015)

La compensazione per l'esclusione dalla partecipazione diretta alle imprese coloniali, in altre parole, avviene non solamente tramite la riesumazione di un passato glorioso, ma anche attraverso il progetto di presentare Venezia come uno dei maggiori centri europei di raccolta e diffusione dell'informazione geografica, costruendo un'immagine della città quale 'specola' del mondo, in cui, nelle parole di Franco Farinelli, «si produceva ed esercitava il massimo dell'intelligence planetaria» $(2007,74)$. Se questo discorso mitopoietico e mitografico, come osserva Peter Burke (2000), di lì a poco registrerà un ripiegamento, un'involuzione verso il racconto autoreferenziale dell'eccezionalità veneziana, ai tempi di Ramusio l'investimento nel capitale strategico e mimetico della rappresentazione geografica appare ancora dei più sostenuti. ${ }^{10} \mathrm{~A}$ tale investimento contribuiscono tanto l'istituzione di ambascerie permanenti, con la conseguente produzione, stratificazione e archiviazione di scritture diplomatiche (relazioni, dispacci, diari privati), e il mantenimento di un sistema postale capace di garantire un costante afflusso di informazioni, ${ }^{11}$ quanto una politica culturale che si traduce non solo in commissioni pubbliche ma orienta e condiziona il mercato dell'imprenditoria culturale privata, in particolare lo sviluppo di una fiorente editoria a carattere geografico e cartografico. Fra gli obiettivi strategici implicitamente sottesi alle Navigazioni, è possibile così riconoscere la celebrazione della capacità del governo e delle élite veneziane di cooptare informazioni geografiche, sottoporle ad analisi scientifica, elaborando nuovi modelli e teorie di funzionamento del mondo. Con questo obiettivo in mente, Ramusio, al contrario di quanto sostenuto da Parks, non si limita a riciclare prodotti già presenti sul mercato librario della città, ma si impegna nel reperimento e nella selezione di materiali

10 In questa direzione, in Meraviglia e possesso, il lavoro dedicato da Stephen Greenblatt alla letteratura di viaggio rinascimentale, la rappresentazione non appare come un riflesso o un prodotto di rapporti sociali, ma è «essa stessa un rapporto sociale, legato alle convinzioni collettive, alle gerarchie di status, alle resistenze e ai conflitti che esistono nelle altre sfere della cultura nel cui ambito essa circola» $(1994,29)$.

11 La cui celerità è testimoniata, oltre che dai Diarii in cui Marin Sanudo registrava quotidianamente l'arrivo di dispacci e novità in Senato, anche dalle lettere, pubblicate da Andrea Del Ben (2004), con cui Ramusio nel 1537 informava Bembo riguardo agli eventi militari in corso nel Mediterraneo orientale, in particolare ai movimenti della flotta ottomana che di lì a poco avrebbero condotto alla disfatta della Lega Santa nella battaglia di Prevesa (1538). Si veda il contributo di Fabio Romanini in questo volume. 
rari o difficilmente accessibili (cf. Veneri 2012a). Alla luce di queste considerazioni, può dunque apparire giustificata la perplessità dimostrata dallo studioso americano di fronte all'estraneità dell'attività cancelleresca di Ramusio alle Navigazioni, così come lo stupore per il fatto che il segretario non avesse pubblicato alcun documento veneziano contemporaneo che poteva avere per le mani, grazie alla pratica quotidiana con ambasciatori e diplomatici (Parks 1955b, 129). È necessario allora considerare con quanta attenzione le autorità sorvegliassero la circolazione di tali informazioni e come fossero proprio i segretari a svolgere un ruolo determinante nel loro controllo. Incaricati della gestione degli archivi (la secreta), i segretari, pur privi di potere decisionale, svolgevano un ruolo consultivo fondamentale, capace di orientare i provvedimenti delle diverse magistrature e degli organi collegiali che assistevano, in particolar modo quelli presi dal Consiglio dei Dieci che di fatto dirigeva la politica estera della Repubblica (Trebbi 1986; Zannini 1993). Inoltre, un momento importante della loro carriera consisteva nell'accompagnare ambasciatori e oratori nelle loro missioni diplomatiche, un compito svolto pure da Ramusio in giovane età - quando si era recato, fra il 1505 e il 1507, in Francia al seguito di Alvise Mocenigo - cui erano collegati imperativi di circospezione e segretezza, come ricorda Giovanni Carlo Scaramelli nel suo memoriale del 1570:

come secretario di Repubblica doverò sapere che 'l carico mio consiste in tre cose: negoziare, servire et tener secrete le cose negoziate et scritte. Si negozia con diverse persone, con l'ambasciator padron proprio, col prencipe a chi si è destinati, con i suoi consiglieri, con altri ambasciatori, et finalmente col rimanente della Corte [...]. Però mi converà esser molto circospeto et con il non creder leggermente ogni cosa et con il non diffidar sempre di ciascuno, tener il negozio in modo bilanciato, ch'io non sia per cader mai in sospetto di poca prudenza o di mala volontà. (Maggio 2001, CXVI-CXVIII)

Tutte queste cautele nella gestione dell'informazione, richieste dalla sua posizione di segretario, sembrano all'origine della scelta non solo di non pubblicare testi veneziani recenti e attuali, ma anche di condurre la celebrazione di Venezia attraverso monumenti il cui valore strategico era ormai andato in prescrizione. La specificità di questa scelta pare ancora più significativa, se si considera che invece Ramusio non si perita di pubblicare documenti ufficiali spagnoli e portoghesi altrettanto controllati e sigillati nei rispettivi imperi di provenienza. In questo caso la reticenza del segretario sta nel tacere l'origine di questi materiali, per la quale sono stati avanzati i nomi di due alti funzionari diplomatici con cui il raccoglitore era in contatto: l'ambasciatore spagnolo a Venezia, Diego Hurtado de Mendoza, e l'ambasciatore veneziano presso l'imperatore, Francesco Contarini, verosimilmente i tramiti attraverso cui Ramusio venne in possesso della 
serie di lettere e relazioni riguardanti le esplorazioni spagnole degli anni Trenta alla frontiera nordoccidentale della Nuova Spagna (Parks 1955b, 144). Quanto ai materiali riguardanti le esplorazioni portoghesi in Africa, va ricordata la polemica che Ramusio, nei discorsi introduttivi alle navigazioni di Alvise da Mosto e di Annone, dirige contro le autorità lusitane, che non solo paiono disinteressate a spingersi nell'entroterra e ad aprire nuovi traffici, ma vietano la circolazione di notizie che potrebbero incrementare tanto le conoscenze geografiche quanto i commerci internazionali:

Ma, sapendo già tanti anni li serenissimi re di Portogallo tutte le sopradette cose, e molte di più, circa detto viaggio e non avendo voluto che fin ad ora sia fatto, è da pensar che sia stato per loro convenienti respetti, li quali, come non è bene di volergli investigare, così ancora penso che non sia lecito il voler discorrer più oltre sopra di molte altre cose di valore e ad uso del vivere nostro, che si potrian cavare di quella parte della Etiopia qual è fra il tropico di Cancro e l'equinoziale, e corre per li medemi paralleli di longitudine che correno le Indie orientali. (Milanesi 1978-88, 1: 470-1)

Lo andarvi è del tutto proibito dai detti re, né vogliono che si sappian né queste né molte altre cose. (561)

Ramusio tenta di aggirare queste proibizioni in vario modo: rintracciando le lettere di Giovanni da Empoli e di Andrea Corsali, recuperando da edizioni a stampa due relazioni e un sommario vespucciano, ma soprattutto dando egli stesso stesura scritta al resoconto della navigazione all'isola di San Tomé, fattogli da un pilotto portoghese, amico del conte Raimondo Della Torre, «il cui nome per convenienti rispetti si tace» (554). Allo stesso modo Ramusio non rivela nemmeno il nome del «gentiluomo portoghese, il qual avea fatto gran fatiche nelle buone lettere, e si dilettava grandemente di cosmografia», che lo aiuta a stabilire la veridicità della navigazione, attraverso il mar Rosso fino a Sumatra, del mercante greco Iambolo (903); così come nel Discorso sopra li viaggi delle spetierie compare «un gentiluomo, grandissimo filosofo e matematico [...] il nome del quale per suoi rispetti non si dice» (2: 979).

Reticente è Ramusio anche circa le transazioni, che rimangono avvolte dal più fitto mistero, attraverso le quali ottiene altri materiali inediti o semiclandestini di cui le Navigazioni rimangono tuttora l'unica attestazione, fra cui il racconto della spedizione di Estevão da Gama redatto dallo scrivano di bordo Tomé Lopez e inviato alle autorità fiorentine. Infine, sono le maglie più permeabili dell'informazione spagnola che sembrano permettere la violazione del 'sigillo' portoghese, lasciando trapelare materiali riguardanti l'Oceano indiano e le Molucche. Del Livro di Duarte Barbosa e della Suma oriental di Tomé Pires Ramusio ci dice che 
poi che da principio furon letti e venuti a notizia di alcune poche persone, sono stati nascosi e non è stato permesso che fussero publicati per convenienti rispetti. (Milanesi 1978-88, 2: 541)

Ramusio, che a riguardo non rivela altro, sottolinea che è stato solo «con grandissima fatica e difficultà» che è riuscito ad averne le trascrizioni, rispettivamente a Siviglia e a Lisbona. La vasta rete di contatti, intrattenuta da Ramusio e da Giunti con vari ambienti geografici europei, emerge così in maniera asistematica nei Discorsi e lascia agli studiosi il compito di ricostruirne le complesse articolazioni a partire dall'esame delle fonti.

\section{Reticenze epistemiche}

Accanto alle reticenze dovute a ragioni di ordine sociale, politico e strategico, è possibile individuare nella configurazione stessa del progetto ramusiano il motivo di alcuni altri eloquenti silenzi. Non può non colpire il fatto che il segretario taccia, o rimanga decisamente vago, circa il vasto materiale cartografico grazie al quale 'spazializzava' l'informazione dei viaggiatori, confrontando, correggendo, aggiungendo valori di longitudine e latitudine. Venezia è all'epoca uno dei maggiori centri dell'editoria cartografica a stampa (Woodward 2002 18; Karrow 2007, 617-8) - ma è anche un importante centro di produzione manoscritta di carte e atlanti nautici, a partire dalla celebre bottega di Battista Agnese. La continua pratica di comparazione fra i toponimi e gradi registrati o suggeriti dai viaggiatori e le mappe che possiamo immaginare sullo scrittoio ramusiano danno luogo a un diffuso sotto-testo cartografico, che permette di leggere le Navigazioni come un vero e proprio progetto europeo di 'mappatura' del mondo. Ora, Ramusio avrebbe facilmente potuto riferirsi ai risultati più avanzati della cartografia a lui contemporanea, così come avrebbe potuto insistere sugli errori e le lacune che le sue informazioni permettevano di correggere. Ma tale rinvio nella raccolta si limita alle tavole tolemaiche e, in maniera molto generica, alle carte nautiche portoghesi. Una possibile spiegazione per questa scarsità di riferimenti cartografici è forse ricavabile dalla dichiarazione d'intenti che Ramusio inserisce all'inizio del primo volume: quella, in fin dei conti, di sostituire Tolomeo, dal momento che con i testi delle Navigazioni e

aggiugnendo la descrizion delle carte marine portoghesi, si potrian fare altretante tavole che sarebbero di grandissima satisfazione a quelli che si dilettano di tal cognizione, perché sarian certi dei gradi, delle larghezze e lunghezze almanco delle marine di tutte queste parti, e de' nomi de luoghi, città e signori che vi abitano al presente, e potriano conferirle con quel tanto che ne hanno scritto gli auttori antichi. (Milanesi 1978-88, 1: 5) 
Ramusio ha dunque in mente la compilazione di nuove carte geografiche che rendano obsolete anche le mappe più avanzate dell'epoca e di conseguenza superfluo il loro richiamo. E in questo sarebbe difficile dargli torto, dal momento che la collaborazione con Giacomo Gastaldi - sulla quale pure Ramusio è reticente - ha prodotto delle mappe che, grazie all'incorporazione di informazioni tratte dalle Navigazioni, si sono rivelate, nel contesto della moderna cartografia europea, fra le più ricche e dettagliate del loro tempo - basti pensare alla carta dell'Asia che uscirà di lì a poco incorporando informazioni tratte da Marco Polo e altri viaggiatori in Oriente o alla carta generale dell'Africa, basata sulle descrizioni di al-Hasan alWazzan, ovvero Leone Africano (Gastaldi 1561, 1564; cf. Romanini 2013, Amadori 2014). Il rigettare 'dietro le quinte' l'uso di fonti cartografiche potrebbe anche obbedire a una scelta di tipo formale, in un momento in cui la codificazione dei generi letterari e scientifici dà sempre più peso alla demarcazione fra generi narrativi e generi descrittivi, fra codici verbali e codici visuali. Da questo punto di vista le Navigazioni si offrono come monumento alla letteratura di viaggio europea e al suo capitale informativo, e la 'nobilitazione' del genere trae vantaggio proprio dalla sua autonomia rispetto ad altre forme di rappresentazione dello spazio geografico. In questa direzione si potrebbe infine leggere la reticenza di Ramusio nei confronti di testi e informazioni geografiche che mal si adattano alla sua peculiare visione spaziale, favole premoderne che difficilmente riescono a tradursi in dati scientifici, come quelle, radicate in una visione misticoreligiosa, che gli aveva proposto nel 1530 il falso messia David Reubeni nel racconto dei suoi viaggi in Africa e nel vicino Oriente (Sanudo 1899, 145-8), o la noiosa epica militare e propagandistica che, a parer di Ramusio, inquina gran parte della storiografia spagnola. ${ }^{12} \mathrm{Si}$ capisce allora perché, ad esempio, uno dei monumenti più celebri della letteratura europea

12 All'inizio del terzo volume, Ramusio ribadisce la propria profonda diversità di preoccupazioni rispetto alla retorica militare della storiografia spagnola, in cui i dati geografici, le osservazioni etnografiche, tutta la storia naturale vengono soffocati da noiosi elenchi di capitani e comandanti e dalla loro «immensa ingordigia dell'oro». In particolare Ramusio deplora la mancata pubblicazione delle due restanti parti della storia delle Indie di Oviedo, nelle quali «v'erano più di 400 figure de' ritratti delle cose naturali, come animali, uccelli, pesci, arbori, erbe, fiori e frutti delle dette due parti dell'Indie. Il che è stato di gran perdita a' studiosi, che desiderano di legger e intender particolarmente e più volentieri le cose sopradette dalla natura prodotte in quelle parti, dissimili da quelle che nascono presso di noi, che di saper le guerre civili ch'hanno fatte molt'anni gli Spagnuoli tra loro, ribellandosi alla maestà cesarea di Carlo V imperatore per l'immensa ingordigia dell'oro. Delle quali guerre tutti gl'istorici spagnuoli di questi tempi s'hanno affaticato e affaticano continuamente di scrivere con un'estrema diligenza, notando che ne' fatti d'arme di Salinas, Chupas, Quito, Guarina, Xaquixaguana v'erano i tali e tali capitani, alfieri e adelantadi, co' nomi di tutti i soldati spagnuoli, sì da cavallo come da piedi, e in qual città di Spagna ciascun di lor nacquero, cosa vana e ridicolosa; delle cose naturali veramente sopradette se ne passano brevemente, se non in quanto non possono far di meno di non nominarle alle fiate» (Milanesi 1978-83, 5: 10). 
tardomedievale, i viaggi di John Mandeville, non trovi posto nella silloge ramusiana, così come venga ignorata la vasta produzione di letteratura di pellegrinaggio, che pure negli ultimi due secoli era andata incontro a un processo di secolarizzazione, trasformando molti itinerari devozionali in complicati peripli ed esplorazioni del vicino Oriente.

È dunque l'assetto descrittivo e narrativo di questi testi, poco funzionale all'estrapolazione di dati geografici, a determinarne l'esclusione dal discorso umanistico condotto da Ramusio, in ragione di una loro intrinseca premodernità o insufficiente scientificità. A questa ragionata esclusione di documenti europei si accompagna una pretesa universalistica - l' 'hybris del punto zero', ben visibile, secondo Walter Mignolo, nella cartografia orteliana $(2011,325)$ - che squalifica invece già dal principio la possibilità, per le cosmologie, le pratiche e i saperi spaziali non europei, di esistere al di fuori o accanto a tale discorso. Alla de-soggettivazione, reificazione e mercificazione degli individui non europei messa in atto dal colonialismo e dal commercio degli schiavi (le teste de Negri la cui vendita Ramusio indica fra i vantaggi possibili della navigazione del fiume Niger) ${ }^{13}$ corrisponde l'oggettivazione, de-territorializzazione e svalutazione dei loro saperi (le imagini ieroglifice messicane di cui riferisce Oviedo, che nell'edizione del 1606 diventano bizzarrie, cf. Milanesi 1978-88, 5: 11). In altre parole, l'umanesimo di Ramusio non si distanzia ma anzi aderisce implicitamente, anche in questo caso con un certo grado di reticenza, al 'razzismo epistemico' eurocentrico, che all'epoca informava e legittimava non solo le politiche di colonizzazione ma costruiva le basi teoriche e ideologiche per lo sviluppo di una 'colonialità' del potere e del sapere, che da quel momento ai giorni nostri non avrebbe smesso di operare e riconfigurarsi (Dussel 1993; Quijano 2010). In questo senso, può apparire sintomatico della posizione reticente del segretario il fatto che la produzione più visibile di 'differenza coloniale' venga affidata alle parole di interlocutori terzi. Ė così che nel discorso sopra la navigazione di Arriano, è un pilotto portoghese a rendere chiaro come la messa in dubbio della teoria degli antipodi non coincida con lo smantellamento delle categorie razziali a essa associate, ma anzi rafforzi l'opposizione fra barbarie e civiltà:

Che veramente li paralleli di sopra l'equinoziale verso di noi corrispondino nella forma e colore degli uomini e degli animali con li paralleli di

13 «È da esistimare che vi concorreria gran numero di mercatanti per il grande utile che vi saria, essendo viaggio così propinquo e non vi andando tanto tempo e spesa come va in quello delle Indie orientali. E oltra l'oro puro e infinito, riporteriano ancora delle loro merci molte teste de Negri, i quali, condotti all'isola di San Iacobo di Capo Verde, si vendono immediate per le Indie occidentali» (Milanesi 1978-83, 1: 470). È possibile inoltre ricordare che Ramusio, nel 1537, aveva stipulato un contratto con Oviedo al fine di istituire una società privata rivolta al traffico di prodotti fra l'Italia, Cadice e Santo Domingo (il contratto è stato pubblicato in Gerbi 1975, 272-8). 
sotto l'equinoziale verso il polo antartico, diceva medesimamente che anco questo in gran parte si vedeva non esser vero, conciosiacosaché 'l parallelo sopra lo stretto di Gibralterra, il quale corre gradi trentacinque e mezzo, corrisponde al parallelo che corre all'opposito sopra il capo di Buona Speranza in gradi trentacinque e mezzo, e nondimeno nel detto stretto gli uomini sono bianchi e civili e di buono ingegno, e nel capo di Buona Speranza sono negri, di grossissimo intelletto e salvatichi quasi come fiere [...]. Affermava ancora aver navigato lungo la costa della terra di Brasil verso il polo antartico, e aver passato quarantacinque gradi e più, dove tutti gli abitanti sono di colore olivastro, e più presto negri, e di costumi crudeli e barbari: e qui da noi in detti gradi oppositi, come è la Lombardia, gli uomini sono bianchi e civili. (Milanesi 1978-88, 2: 504-5)

Allo stesso modo, l'importanza del nesso strategico che collega sapere geografico e politiche coloniali di conquista, evangelizzazione, 'addomesticamento' culturale e sfruttamento economico, è efficacemente riassunto nelle parole dell'altro illustre personaggio (l'anonimo di Caffi'), che ragiona con Ramusio, Sanmicheli e Fracastoro nella villa di quest'ultimo:

Si maravigliava fuor di misura come non sia ricordato alli principi grandi, alli quali Iddio ha deputato questa cura, e tengono sempre alli consigli loro uomini grandi sì di lettere come d'intelletto, ch'una delle più ammirabili e stupende operazioni che potessero far in vita loro saria il far conoscere insieme gli uomini di questo nostro emispero con quelli dell'altro opposito, dove sariano reputati per dei, sì come ebbero gli antichi Ercole e Alessandro, che passorono solamente nell'India, e che 'l titolo di questa impresa avanzeria di gran lunga e senza alcun parangone tutti quelli di Giulio Cesare e di ciascun altro imperador romano. La qual cosa potriano fare facilmente mandando in diversi luoghi del detto emispero colonie ad abitarvi, nel modo che faceano i Romani nelle provincie di nuovo acquistate, le quali a poco a poco andassero scoprendo quelle parti, coltivandole e introducendovi la civiltà, e da valenti uomini poi farvi predicar la fede di nostro Signor Giesù Cristo; e per domesticarli più facilmente vi facessero andar ogni anno delle navi cariche di farine, vini, spezie, zuccari e altre sorti di mercanzie di queste nostre parti, all'incontro delle quali non è dubio alcuno che riportariano da quei popoli infinito oro e argento. (Milanesi 1978-88, 2: 980)

\section{Le curvature biocentriche del discorso ramusiano}

Alla luce di questi ultimi passaggi, le osservazioni fin qui formulate sulle reticenze ramusiane possono aiutare a ridefinire il 'luogo di enunciazione' dei suoi discorsi, e a rispondere alle domande 'epistemicamente disobbe- 
dienti' già indirizzate da Mignolo a Kant in una lettura decoloniale della Geografia del filosofo tedesco $(2011,325)$. Da questo punto di vista, si è potuto in primo luogo vedere come l'identità del soggetto conoscitore e il suo apparato materiale di enunciazione rimandino a un contesto sociale (i cittadini originari, la cancelleria), intellettuale (i circoli umanistici) ed economico (l'industria editoriale veneziana) preciso e condizionato tanto da rigide gerarchie quanto da margini aperti all'affermazione individuale. In secondo luogo, il tipo di conoscenza generata dal soggetto conoscitore e le ragioni di tale generazione si pongono tanto nell'ottica umanistica europea di un superamento della conoscenza degli antichi, ovvero nella costruzione di una scienza geografica e cartografica moderna, quanto all'interno di un programma ideologico e politico volto a valorizzare il ruolo di Venezia nella produzione di tale scienza (nel passato: Marco Polo e il canone di ambasciatori-mercanti; nel presente: le Navigazioni stesse). In terzo luogo, i beneficiari di tale conoscenza vengono individuati da Ramusio stesso nelle duplici fila - non esclusivamente italiane, ma certamente europee - di un pubblico erudito (dotti e studiosi), la cui sete di sapere le Navigazioni si propongono di soddisfare, e di una classe politica di governo impegnata nell'espansione coloniale e nel commercio internazionale (signori e principi), cui la raccolta potrebbe fornire un attendibile e agevole strumento di lavoro e progettazione (Milanesi 1978-88, 1: 5). Infine, riguardo alle istituzioni che sostengono e promuovono tale conoscenza, se appare ormai chiaro il coinvolgimento su larga scala del governo veneziano, va altresì ricordata la rete di relazioni, pubbliche e private, politiche ed editoriali, grazie alle quali Ramusio è riuscito a raccogliere e selezionare nel corso di molti anni i materiali e documenti per la sua raccolta.

Rimangono infine da sottolineare quali sono, in linea generale e alla luce di tali reticenze, le occasioni in cui, nei Discorsi che scandiscono le Navigazioni, Ramusio si espone in prima persona. Occasioni tanto più degne di nota, quanto più questi paratesti esibiscono un alto livello di elaborazione retorica, come in più casi ha confermato l'analisi linguistica di Fabio Romanini (2007, 283-4). Per usare i termini impiegati da Eugenio Burgio (2015) in merito al libro di Marco Polo, la cui fortuna si deve anche a una particolare tensione interna fra «trattato» e «verità biocentrica», anche il discorso di Ramusio ricorre in diverse occasioni a una certa «curvatura biocentrica» al fine di sostenere la verità della descrizione. Ciò che è interessante è che in questa serie di 'aneddoti', Ramusio, più che protagonista e fonte diretta per una comprova autoptica, figura come ricevente e latore di notizie non ancora formalizzate dalla scrittura. Nel primo volume, è il caso delle notizie riguardanti al-Hasan al-Wazzan (Leone Africano) «della cui vita - dice Ramusio - dirò quello che ne ho ritratto da persone degne di fede, che nella città di Roma l'han conosciuto e praticato» (Milanesi 197888, 1: 5); così come delle informazioni che, racconta ancora il segretario, «altre volte io notai in certi miei memoriali, avendole udite ragionare da un 
gentil pilotto portoghese di Villa di Condi» (554); di quando Ramusio, trattando della navigazione di Iambolo, annuncia di voler «raccontar quanto sopra quella udì altre volte parlarne da un gentiluomo portoghese» (903); di quando riferisce quello che riguardo all'Africa australe «io udì altre volte parlarne molto lungamente un piloto portoghese, che aveva cognizione de' libri di Tolomeo» (503); o ancora del

grande e ammirabile ragionamento che io udi' questi mesi passati, insieme coll'eccellente architetto messer Michele da San Michele, nell'ameno e dilettevol luogo dell'eccellente messer Ieronimo Fracastoro detto Cafi, posto nel Veronese, sopra la sommità di un colle che discopre tutto il lago di Garda. Il qual ragionamento non mi basta l'animo di poter scriver così particolarmente com'io lo udi', perché mi saria di bisogno d'altro ingegno e altra memoria che non è la mia; pur mi sforzerò sommariamente e come per capi di recitar quel che mi potrò ricordare. In questo luogo di Cafi adunque essendo andati a visitar detto eccellente messer Ieronimo, lo trovammo accompagnato con un gentiluomo, grandissimo filosofo e matematico, che allora gli mostrava uno instrumento fatto sopra un moto de' cieli trovato di nuovo, il nome del quale per suoi rispetti non si dice. (2: 979)

Menzionata a scopo di autenticazione e validazione è anche, nel secondo volume, la fonte orale dichiarata del 'romanzetto' di Marco Polo, «quello ch'io essendo giovanetto n'ho udito molte fiate dire dal clarissimo messer Gasparo Malipiero [...] che riferiva d'averlo inteso ancor lui da suo padre e avo, e d'alcuni altri vecchi uomini suoi vicini» (3: 29); nella stessa direzione sembra andare la trascrizione di quanto Ramusio già intese «da un uomo persiano di molto bello ingegno e giudicio» (60), ovvero il già ricordato Chaggi Memet; così come le cose che Ramusio «già essendo giovane udì piú volte dire dal molto dotto e reverendo don Paolo Orlandino di Firenze» sopra il mappamondo di fra Mauro conservato nel monastero di San Michele in Isola (68).

I casi in cui Ramusio si esprime in prima persona non si limitano però a questi aneddoti e sono anzi molto più numerosi. Essi sembrano rispondere ad almeno tre intenzioni: in primo luogo, quella di porre la nascita e lo sviluppo dell'impresa scientifica ed editoriale delle Navigazioni sotto il nome di autorevoli amicizie, confermando la scelta di un profilo arretrato; in secondo luogo, quella di rimarcare la complessità e la difficoltà delle operazioni di reperimento ed edizione dei testi, più ancora che per esaltare il proprio lavoro, per attribuire valore ai testi pubblicati - testi rari, proibiti 
o corrotti dal tempo; ${ }^{14}$ infine, Ramusio prende direttamente la parola, spesso con domande retoriche, al fine di guidare la lettura dei testi secondo precise angolature strategiche.$^{15} \mathrm{E}$ in questi passaggi che meglio emerge il punto di vista si potrebbe dire 'sopraelevato' di Ramusio, quello che gli permette di abbracciare complessivamente gli assi dei traffici commerciali e le dinamiche geografiche degli espansionismi imperiali e coloniali. Una visione organica e aggiornata, quale la si poteva elaborare solo da una posizione che si voleva, come si è già detto, uno dei centri massimi dell'intelligence planetaria, Palazzo Ducale, il cui ruolo viene comprensibilmente, 'per convenienti rispetti', taciuto. Da queste osservazioni appare chiaro come le molte 'reticenze' di Ramusio obbediscano a una precisa economia discorsiva, in cui la 'curvatura biocentrica' è costantemente controllata e regolata da almeno due fattori: da una parte, l'esigenza scientifica e retorica di avvalorare l'attendibilità dei testi presentati; dall'altra, l'obbligo di circospezione e segretezza cui Ramusio, per la sua posizione cancelleresca, non poteva sottrarsi.

14 Bastino qui due esempi tratti dal primo volume delle Navigazioni. Circa la descrizione dell'Africa di al-Hasan al-Wazzan (Leone Africano), Ramusio riferisce che «gli esemplari che mi son venuti alle mani erano estremamente guasti e scorretti, cosa che averia sbigottito ogni forte e gagliardo intelletto [...] il qual libro scritto da lui medesimo dopo molti accidenti che sariano lunghi a raccontare, pervenne nelle nostre mani; e noi con quella maggior diligenza che abbiamo potuto ci siamo ingegnati con ogni fedeltà di farlo venire in luce, nel modo che ora si legge» (Milanesi 1978-88, 1: 6). Similmente, riguardo all'Itinerario di Ludovico Varthema, il segretario racconta come sia "stato molti anni letto con infiniti errori e incorrezioni; e ancor nell'avvenir così si leggeria, se 'l nostro Signor Iddio non ne avesse fatto venir alle mani un libro de un Cristoforo di Arco, clerico di Sibillia, il quale, avendo avuto un esemplar latino di detto viaggio, tratto dal proprio originale dirizzato al reverendissimo cardinal Carvaial di Santa Croce, lo tradusse in lingua spagnuola con gran diligenzia» (761).

15 Di seguito, alcuni esempi, anch'essi tratti dal primo volume della raccolta: «Ma che dico io del piacere che ne aranno li dotti e studiosi? Chi è colui che possa dubitare che ancor molti dei signori e principi non si abbiano a dilettare di cosí fatta lezione?» (5); «E che bisogna dir? La commodità e facilità che saria a condur ogni sorte di mercatanzia per il detto fiume del Niger, che è grossissimo come il Nilo e si può navigar per cinquecento e più miglia, trovando sempre città e regni? Appresso, quanto guadagno si faria conducendovi il sale, tanto caro e apprezzato da loro?» (470); «E che bisogna dire? Non si vede che fino a' nostri giorni per mancamento di memoria la metà del mondo verso ponente, detta l'Indie occidentali, tanto abitata e piena di genti, era incognita (ancor che Platon dica che gli antichi Egizii ne avean cognizione), se 'l nostro Signor Iesù Cristo non l'avesse fatta scoprire, per esaltare in quella il suo santissimo nome?» (600). 


\section{Bibliografia generale}

Alcover i Sureda, Antoni Maria; de Borja Moll, Francesc (1962). Diccionari català-valencià-barlear, Institut d'Estudis Catalans. Palma de Mallorca: Moll. URL http://dcvb.iec.cat/ (2017-10-25).

Amadori, Gabriele (2014). Giovanni Leone Africano: Cosmographia de l'Affrica. Roma: Aracne Editrice.

Amatucci, Eleonora (1982-3). La redazione toscana B del "Milione" di Marco Polo: edizione critica [tesi di laurea]. Firenze: Università degli Studi di Firenze.

Andreose, Alvise (2002). «La prima attestazione della versione VA del Milione (ms. 3999 della Biblioteca Casanatense di Roma). Studio linguistico». Critica del testo, 5, 655-68.

Andreose, Alvise (2015a). «Le fonti di Ramusio e il loro trattamento nella compilazione de I Viaggi di Messer Marco Polo. Preliminari». Simion, Burgio 2015. URL http://virgo.unive.it/ecf-workflow/books/Ramusio/ main/intro_03.html\#Preliminari (2017-12-18).

Andreose, Alvise (2015b). «Marco Polo's Devisement dou monde and FrancoItalian tradition». Francigena, 1, 261-91. [https://www. francigena-unipd. com/index.php/francigena/article/view/6] (2017-11-09).

Andreose, Alvise (2015c). «Il Devisement dou monde e il progetto editoriale di Rustichello da Pisa». Barbieri, Alvaro; Gregori, Elisa (a cura di), L'autorialità plurima. Scritture collettive, testi a più mani, opere a firma multipla = Atti del XLII Convegno Interuniversitario (Bressanone, 10-13 luglio 2014). Padova: Esedra, 443-60. Quaderni del Circolo Filologico Linguistico Padovano 30.

Andreose, Alvise (2015d). «Marco Polo e il ponte sul fiume Pulisanghin». Cepraga, Dan Octavian; Gumenai, Ion; Şipoş, Sorin (a cura di), Tradiţii istorice romaneşti şi perspective europene. In honorem academician IoanAurel Pop. Oradea: Editura Universităţii din Oradea; Chişinau: Editura Universităţii de Stat din Moldova, 122-36.

Andreose, Alvise (2018). «Il greco di Marco Polo». Andreose, Alvise; Borriero, Giovanni; Zanon, Tobia (a cura di), "La somma de le cose". Studi in onore di Gianfelice Peron. Padova: Esedra, 127-36.

Andreose, Alvise (in corso di stampa). «Between Text and History: Marco Polo's Description of the Lugou Qiao (卢沟桥)». Rong, Xinjiang; Dang, Baohai (eds), Marco Polo and the Silk Road (10th-14th Centuries) = Proceedings of the International Conference (International Academy for China Studies of Peking University, November 19th-20th, 2016). 
Andreose, Alvise; Concina, Chiara (2016). «A monte di F e f. Il Devisement dou monde e la scripta dei manoscritti francesi di origine pisano-genovese». Pioletti, Alberto; Rapisarda, Stefano (a cura di), Forme letterarie del Medioevo romanzo: testo, interpretazione e storia = Atti dell'XI Congresso Società Italiana di Filologia Romanza (Catania, 22-26 settembre 2015). Soveria Mannelli: Rubbettino, 15-37.

Andrews, Tara Lee (2013). «The Third Way: Philology and Critical Edition in the Digital Age». Variants, 10, 61-76. URL https://lirias.kuleuven. be/bitstream/123456789/352304/2/ (2018-01-05).

Anghiera, Pietro Martire di; Oviedo y Valdés, Gonzalo Fernández de (1534). Summario de la generale historia de l'Indie Occidentali. Venezia: [Giunti]. Atwood, Christopher P. (2004). Encyclopedia of Mongolia and the Mongol empire. New York: Facts on File.

Badamxatan, Sandagsürengiyn (1986). «Les chamanistes du Buddha vivant». Études mongoles et sibériennes, 17, 1-214.

Banzarov, Dordži (1891). Černaja Vera ili šamanstvo u mongolov i drugija stat'i. A cura di G.N. Potanin. Sanktpeterburg. Tipografija Imperatorskoj Akademij Nauki. Imp. Akad. Nauk.

Barbieri, Alvaro (a cura di) (1998). Marco Polo: Milione. Redazione latina del manoscritto Z. Parma: Fondazione Pietro Bembo; Guanda.

Barbieri, Alvaro (2004). Dal viaggio al libro. Studi sul "Milione". Verona: Fiorini. Medioevi, Studi 6.

Barbieri, Alvaro (2011). «Introduzione, par. 1». Burgio 2011, VII-XVII.

Barbieri, Alvaro (2015). «I Viaggi di Messer Marco Polo di Giovanni Battista Ramusio: preliminari». Simion, Burgio 2015. URL http://virgo.unive. it/ecf-workflow/books/Ramusio/main/index.html (2018-02-17).

Barbieri, Alvaro; Andreose, Alvise (a cura di) (1999). Marco Polo: Il Milione veneto. Ms. CM 211 della Biblioteca civica di Padova. Venezia: Marsilio.

Barzman, Karen-edis (2014). «Cartographic Line and the 'Paper Management' of the Early Modern State: A Case Study of Venetian Dalmatia». Mapline, $122,1-12$.

Baskakov, NikolajA. (1953).Xakassko-RusskijSlovar'. Moskva: Gosudarstvennoe Izdatel'stvo Inostrannyx i Nacional'nyx Slovarej.

Battaglia Ricci, Lucia (a cura di) (2001). Marco Polo: Milione. Firenze: Sansoni.

Battaglia, Salvatore (a cura di) (1961-2002). Grande Dizionario della Lingua Italiana. 20 voll. Torino: UTET.

Benedetto, Luigi Foscolo (a cura di) (1928). Marco Polo: Il Milione. Prima edizione integrale. Firenze: Leo S. Olschki Editore.

Benedetto, Luigi Foscolo (1929). Filologia e Geografia. Critica di una critica. Firenze: Tipografia Editrice Mariano Ricci.

Benedetto, Luigi Foscolo (a cura di) (1932). Il libro di Messer Marco Polo Cittadino di Venezia detto "Milione" dove si raccontano Le Meraviglie 
del Mondo. Ricostruito criticamente e per la prima volta integralmente tradotto in lingua italiana. Milano-Roma: Treves, Treccani, Tumminelli.

Benedetto, Luigi Foscolo (1939). «Nota marcopoliana. A proposito del codice Ghisi». Rendiconti della R. Accademia d'Italia. Classe di scienze morali $e$ storiche, s. 7, 1(1-5), 15-45.

Benedetto, Luigi Foscolo (1959-60). «Ancora qualche rilievo circa la scoperta dello Z toledano». Atti della Accademia delle Scienze di Torino. II, Classe di scienze morali, storiche e filologiche, 94, 519-78.

Bertolucci Pizzorusso, Valeria (1975). Marco Polo: Milione. Versione toscana del Trecento. Edizione a cura di Valeria Bertolucci Pizzorusso. Indice ragionato a cura di Giorgio Raimondo Cardona. Milano: Adelphi.

Bertolucci Pizzorusso, Valeria (1984). «À propos de Marco Polo et de son livre: quelques suggestions de travail». Essor et fortune de la chanson de geste dans l'Europe et l'Orient latin = Actes du 9e Congrès International de la Société Rencesvals pour l'étude des épopées romanes (Padue-Venise, 20 août-4 septembre 1982), vol. 2. Modena: Mucchi, 795-801.

Bertolucci Pizzorusso, Valeria [1977] (2011a). «Enunciazione e produzione del testo nel Milione». Bertolucci Pizzorusso 2011c, 27-67. Or. ed.: Studi mediolatini e volgari, 25, 1977, 5-43.

Bertolucci Pizzorusso, Valeria [1994] (2011b). «Nuovi studi su Marco Polo e Rustichello da Pisa». Bertolucci Pizzorusso 2011c, 109-26. Or. ed.: Morini, Luigina (a cura di) (2001). La cultura dell'Italia padana e la presenza francese nei secoli XIII-XV = Atti del Convegno di Pavia (11-14 settembre 1994). Alessandria: Edizioni dell'Orso, 95-110.

Bertoni, Giulio (1928). «Marco Polo: Milione, di Benedetto, Luigi Foscolo». Giornale Storico della Letteratura Italiana, 92, 285-93.

Bettarini Bruni, Anna; Trovato, Paolo (2009). «Dittico per Antonio Pucci». Filologia italiana, 6, 81-128.

Boerio, Giuseppe (1867). Dizionario del dialetto veneziano. Venezia: Reale tipografia di G. Cecchini.

Bolòs, Jordi; Sànchez-Boira, Imma (2014). Inventaris i encants conservats a l'Arxiu Capitular de Lleida. Barcelona: Fundació Noguera.

Bretschneider, Emil (1876). Archaeological and Historical Researches on Peking and Its Environs. Shanghai: American Presbyterian Mission Press. Burckhardt, Jacob (1955). La civiltà del Rinascimento in Italia. Trad. di Domenico Valbusa. Firenze: Sansoni. Trad. di: Die Kultur der Renaissance in Italien. Basel: Schweighauser, 1860.

Burgio, Eugenio (2008). «Una nota per il Milione: 'trejes' / 'sli(o)zola' ('slitta')». Giachino, Monica; Rusi, Michela; Tamiozzo Goldmann, Silvana (a cura di), La passione impressa. Studi offerti a Anco Marzio Mutterle. Venezia: Cafoscarina, 47-73.

Burgio, Eugenio (2009). «'Cartografie' del viaggio. Sulle relazioni fra la Mappamundi di Fra Mauro e il Milione». Critica del testo, 12(1), 59-106. 
Burgio, Eugenio (a cura di) (2011). Giovanni Battista Ramusio 'editor' del "Milione". Trattamento del testo e manipolazione dei modellli = Atti del Seminario di ricerca (Venezia, 9-10 settembre 2010). Roma; Padova: Antenore. Biblioteca veneta poliana, Documenti per l'edizione integrale del Libro di Marco Polo 3.

Burgio, Eugenio (2014). «Achbaluch, 'nella provincia del Cataio'. (Ramusio, I Viaggi di Messer Marco Polo, II 28, 6-7)». Canettieri, Paolo; Punzi, Arianna (a cura di), Dai pochi ai molti. Studi in onore di Roberto Antonelli, vol. 1. Roma: Viella, 359-73.

Burgio, Eugenio (2015). «Ramusio e la tradizione del Milione». Simion, Burgio 2015. URL http://virgo.unive.it/ecf-workflow/books/Ramusio/ main/intro 02.html (2018-02-17).

Burgio, Eugenio (2017). «Tra Aden e Alessandria. Sull'esistenza di varianti d'autore nel Milione». Divizia, Paolo; Pericoli, Lisa (a cura di), Il Viaggio del testo = Atti del Convegno Internazionale di Filologia italiana e romanza (Brno, 19-21 giugno 2014). Alessandria: Edizioni dell'Orso, 3-22.

Burgio, Eugenio (a cura di) (in corso di stampa). Liber qui vulgari hominum dicitur Elmeliole o Liber domini Marchi Paulo de Venetiis. Epitome latina L.

Burgio, Eugenio; Mascherpa, Giuseppe (2007). «Milione latino. Note linguistiche e appunti di storia della tradizione sulle redazioni Z e L». Oniga, Renato; Vatteroni, Sergio (a cura di), Plurilinguismo letterario. Soveria Mannelli: Rubbettino, 119-58.

Burgio, Eugenio; Eusebi, Mario (2008). «Per una nuova edizione del Milione». Conte 2008, 17-48.

Burgio, Eugenio; Buzzoni, Marina; Ghersetti, Antonella (2012). «A Digital Edition of Dei Viaggi di Messer Marco Polo, Gentilhuomo Venetiano (Giovanni Battista Ramusio, Navigationi et Viaggi, II, 1559): The Project and Its Recent Updates». Quaderni veneti, 1(2), 227-33. DOI 10.14277/1724188X/QV-1-2-12-10.

Burke, Peter (2000). «Early Modern Venice as a Center of Information and Communication». Martin, John; Romano, Dennis (eds), Venice Reconsidered. The History and Civilization of an Italian City-State, 12971797. Baltimore; London: The Johns Hopkins University Press, 389-419.

Buzzoni, Marina (2011). "The "Electronic Hêliand Project": theoretical and practical updates». Cotticelli Curras, Paola (a cura di), Linguistica e filologia digitale: aspetti e progetti. Alessandria: Edizioni dell'Orso, 55-67.

Buzzoni, Marina (2016). «A Protocol for Scholarly Digital Editions? The Italian Point of View». Driscoll, Pierazzo 2016, 59-82. URL https://www. openbookpublishers.com/product/483 (2017-11-09).

Buzzoni, Marina; Burgio, Eugenio (2014). «The Italian Third Way of Editing between Globalization and Localization». Stolz, Michael; Chen, Yen-Chun (Hrsg.), Internationalität und Interdisziplinarität der Editionswissenschaft. Berlin; Boston: De Gruyter, 38, 171-80. 
Buzzoni, Marina; Burgio, Eugenio; Modena, Martina; Simion, Samuela (2016). «Open versus Closed Recensions (Pasquali): Pros and Cons of Some Methods for Computer-Assisted Stemmatology». Digital Scholarship in the Humanities, 31, 652-69.

Cardona, Giorgio Raimondo (1975). «Indice ragionato». Bertolucci Pizzorusso 1975, 488-761.

Casella, Mario (1929). «Il libro di Marco Polo». Archivio storico italiano, s. 7, 11(87), 193-230.

Cattaneo, Angelo (2003). «Fra Mauro cosmographus incomparabilis and his mappamundi: Documents, Sources, and Protocols for Mapping». Curto, Diogo Ramada; Cattaneo, Angelo; Almeida, André Ferrand (a cura di), La cartografia europea tra primo Rinascimento e fine dell'Illuminismo = Atti del Convegno internazionale "The making of European Cartography" (Firenze, BNCF-EUI, 13-15 dicembre 2001). Firenze: Leo S. Olschki Editore, 19-48.

Cattaneo, Angelo (2004-05). «Scritture di viaggio e scrittura cartografica. La mappamundi di Fra Mauro e i racconti di Marco Polo e Niccolò de' Conti». Itineraria, 3-4, 157-202.

Cattaneo, Angelo (2005). La 'mappamundi' di Fra Mauro camaldolese. Venezia, 1450 [PhD Dissertation]. Firenze: European University Institute.

Cattaneo, Angelo (2011). Fra Mauro's "Mappa Mundi” and Fifteenth-Century Venice. Turnhout: Brepols. Terrarum Orbis 8.

Čeremisov, Konstantin M. (1973). Burjatsko-russkij slovar'. Moskva: Sovetskaja Énciklopedija.

Ceresa, Marco (2015). Pulisangan. Simion, Burgio 2015. URL http://virgo. unive.it/ecf-workflow/books/Ramusio/lemmi/Pulisangan.html (201712-15).

Cerquiglini, Bernard (1989). Éloge de la variante. Paris: Seuil.

Certeau, Michel de (1990). L'invention du quotidien 1. Arts de faire. Paris, Gallimard.

Chiesa, Paolo (a cura di) (2011). Guglielmo di Rubruk: Viaggio in Mongolia (Itinerarium). Milano: Fondazione Lorenzo Valla; Arnoldo Mondadori Editore.

Choi, Han-Woo (1992). On the Turkic shamanic word bögü. Bethlenfalvy, Géza; Birtalan, Ágnes; Sárközi, Alice; Vinkovics, Judit (eds), Altaic Religious Beliefs and Practices = Proceedings of the 33rd Meeting of the Permanent International Altaistic Conference (Budapest, June 24-29, 1990). Budapest: Research Group for Altaic Studies, Hungarian Academy of Sciences, 83-7.

Cicogna, Emmanuele Antonio (1824-53). Delle inscrizioni veneziane raccolte e illustrate da Emmanuele Antonio Cicogna cittadino veneto. 6 voll. Venezia: presso vari editori. Vol. 6, presso la tip. Andreola.

Clauson, Sir Gerard (1972). An Etymological Dictionary of Pre-ThirteenthCentury Turkish. Oxford: Clarendon. 
Clough, Cecil H. (1967-72). «A portion of Pietro Bembo's Epistolario». Bodleian Library Record, 8, 26-40.

Coblin, W. South (2007). A Handbook of 'Phags-pa Chinese. Honolulu: University of Hawai'i Press.

Concina, Chiara (2007). «Prime indagini su un nuovo frammento francoveneto del Milione di Marco Polo». Romania, 125, 342-69.

Contarini, Gasparo (1544). La Repubblica e i magistrati di Vinegia. Venezia: Girolamo Scotto.

Corpus OVI. Corpus OVI dell'Italiano antico. Istituto Opera del Vocabolario Italiano. URL http://wWw.ovi.cnr.it/index.php/it/ (2017-10-25).

Cortelazzo, Manlio; Zolli, Paolo (1999). Il nuovo etimologico. DELI Dizionario Etimologico della Lingua Italiana. 2a ed. in vol. unico. Bologna: Zanichelli.

Daffinà, Paolo; Leonardi, Claudio; Lungarotti, Maria Cristina; Menestò, Enrico; Petech, Luciano (a cura di) (1989). Giovanni di Pian di Carpine: Storia dei mongoli. Spoleto: CISAM.

Dancette, Jeanne (1989). «La faute de sens en traduction». TTR: traduction, terminologie, rédaction, 2(2), 83-102.

Dankoff, Robert; Kelly, James (eds) (1982). Maḥmūd al-Kāšğarī: Compendium of the Turkic Dialects (Diwān Luyāt at-Turk). Duxbury (MA): Harvard University. Sources of Oriental Languages \& Literatures 7, Turkish Sources VII/I.

De Curzon, Henri (1886). La règle du temple. Paris: Société de l'Histoire de France.

De Kerckhove, Derrick (2008). Dall'alfabeto a internet. L'homme littéré': alfabetizzazione, cultura, tecnologia. Sesto San Giovanni (MI): Mimesis.

Del Ben, Andrea (1994-5). «La prima edizione della Storia Veneta del Bembo e una lettera inedita di G.B. Ramusio». Atti e Memorie dell'Accademia Patavina di Scienze, Lettere ed Arti, parte III. Memorie della Classe di Scienze Morali, Lettere e Arti, 107, 203-16.

Del Ben, Andrea (2004). Giovanni Battista Ramusio cancelliere e umanista. Con l'edizione di quarantacinque lettere al Bembo. Trieste: Imprinta.

Del Ben, Andrea (2006). Giovanni Battista Ramusio cancelliere e umanista. Con l'edizione di quarantacinque lettere a Pietro Bembo (ms. Ambrosiano D 335 inf.). 2a ed. Trieste: Edizioni Goliardiche.

Del Piero, Antonio (1902). Della vita e degli studi di Gio. Battista Ramusio. Venezia: Visentini.

de Rachewiltz, Igor (1971). Papal Envoys to the Great Khans. London: Faber $\&$ Faber.

de Rachewiltz, Igor (2004). The Secret History of the Mongols: a Mongolian Epic Chronicle of the Thirteenth Century. Translated with a Historical and Philological Commentary by Igor de Rachewiltz. 2 vols. Leiden; Boston: Brill. 
de Rachewiltz, Igor (2006). The Secret History of the Mongols: A Mongolian Epic Chronicle of the Thirteenth Century. 2 vols. Leiden; Boston: Brill.

Devos, Paul (1948). «Le miracle posthume de saint Thomas l'apôtre». Analecta Bollandiana, 66, 231-75.

Diószegi, Vilmos (1963). «Ethnogenic Aspects of Darkhad shamanism». Acta Orientalia Academiae Scientiarum Hungariae, 16, 55-81.

Doerfer, Gerhard (1963). Türkische und mongolische Elemente im Neupersischen I. Wiesbaden: Franz Steiner.

Donattini, Massimo (1980). «Giovanni Battista Ramusio e le sue Navigationi. Appunti per una biografia». Critica storica, 17, 55-100.

Donattini, Massimo (1986). «Una famiglia riminese nella società e cultura veneziane: i Ramusio». Bolognesi, Dante (a cura di), Ravenna in età veneziana. Ravenna: Longo, 279-94.

Donattini, Massimo (1992). «Orizzonti geografici dell'editoria italiana (14931560)». Prosperi, Adriano; Reinhard, Wolfgang (a cura di), Il nuovo mondo nella coscienza italiana e tedesca del Cinquecento. Bologna: il Mulino, 79-154.

Donattini, Massimo (2007). «Etica personale, promozione sociale e memorie di famiglia nella Venezia del Rinascimento. Note su Paolo Ramusio seniore (1443?-1506)». Brizzi, Gian Paolo; Olmi, Giuseppe (a cura di), Dai cantieri della storia. Liber amicorum per Paolo Prodi. Bologna: CLUEB, 317-29.

Driscoll, Matthew James; Pierazzo, Elena (a cura di) (2016a). Digital Scholarly Editing. Theories and Practices. Cambridge: OpenBook Publishers. URL https://WWW.openbookpublishers.com/product/483 (2017-11-09).

Driscoll, Matthew J.; Pierazzo, Elena (2016b). «Introduction: Old Wine in New Bottles?». Driscoll, Pierazzo 2016a, 1-15.

Dussel, Enrique (1993). «Eurocentrism and Modernity». Boundary 2, 20(3), 65-76.

Dutschke, Consuelo W. (1993). Francesco Pipino and the Manuscripts of Marco Polo's "Travels" [tesi di dottorato]. Los Angeles: University of California.

Eliade, Mircea (1964). Shamanism: Archaic Techniques of Ecstasy. Eng. transl. by Willard R. Trask. Princeton (NJ): Princeton University Press.

Erman, Adolph (1850). Travels in Siberia. Philadelphia: Lea \& Blanchard.

Eusebi, Mario [2010] (2018). Marco Polo: Le Devisement dou monde. Testo secondo la lezione del codice fr. 1116 della Bibliothèque Nationale de France. Venezia: Edizioni Ca' Foscari. DOI 10.30687/978-88-6969-223-9.

Falchetta, Piero (2006). Fra Mauro's World Map. Turnhout: Brepols.

Falqui, Enrico; Prati, Angelico (1937). Dizionario di marina medievale e moderno. Roma: Reale Accademia d'Italia.

Farinelli, Franco (2003). Geografia. Un'introduzione ai modelli del mondo. Torino: Einaudi.

Farinelli, Franco (2007). L'invenzione della terra. Palermo: Sellerio. 
Fennis, Jan (1995). Trésor du langage des galères. Dictionnaire exhaustif, avec une introduction, des dessins originaux de René Burlet et des planches de Jean-Antoine de Barras de la Penne, un relevé onomasiologique et une bibliographie. Tübingen: Max Niemeyer Verlag.

FEW = von Wartburg, Walter et al. (1928-). Französisches etymologisches Wörterbuch. URL https://apps.atilf.fr/lecteurFEW/index.php/page/ view (2017-10-25).

Fiormonte, Domenico (2003). Scrittura e filologia nell'era digitale. Torino: Bollati-Boringhieri.

Folena, Gianfranco (1991). Il linguaggio del caos. Studi sul plurilinguismo rinascimentale. Torino: Bollati Boringhieri.

Formisano, Luciano (a cura di) (2006). Iddio ci dia buon viaggio e guadagno. Firenze, Biblioteca Riccardiana, ms. 1910 (Codice Vaglienti). Firenze: Polistampa.

Gadrat, Christine (2010). «Le rôle de Venise dans la diffusion du livre de Marco Polo (XIVe-début XVIe siècle)». Médiévales, 58, 63-78.

Gadrat-Ouerfelli, Christine (2015). Lire Marco Polo au Moyen Age. Traduction, diffusion et réception du "Devisement du Monde". Turnhout: Brepols. Terrarum Orbis, 12.

Gallo, Rodolfo (1943). «Le mappe geografiche del Palazzo Ducale di Venezia». Archivio Veneto, 32(3), 47-113.

Gasparrini Leporace, Tullia (a cura di) (1956). Il "Mappamondo" di Fra Mauro. Presentazione di Roberto Almagià. Roma: Istituto Poligrafico dello Stato.

Gastaldi, Giacomo (1561). Il Disegno della terza parte dell'Asia. [Venezia]: Fabio Licinio.

Gastaldi, Giacomo (1564). Il disegno della geografia moderna de tutta la parte dell'Africa. Venezia: s.n.

Gennari, Pamela (2008-09). "Milione", redazione VB. Edizione critica commentata [tesi di dottorato]. Venezia: Università Ca' Foscari. URL http://dspace.unive.it/handle/10579/937 (2018-09-28).

Georg, Stefan (2001). «Türkisch/Mongolisch tengri 'Himmel, Gott' und seine Herkunft». Studia Etymologica Cracoviensia, 6, 83-100.

Gerbi, Antonello (1975). La natura delle Indie Nove. Da Cristoforo Colombo a Gonzalo Fernandez de Oviedo. Milano; Napoli: Ricciardi.

Gernet, Jacques (1959). La vie quotidienne en Chine à la veille de l'invasion mongole (1250-1276). Paris: Hachette.

Gobbato, Veronica (2015). «Un caso precoce di tradizione indiretta del Milione di Marco Polo: il Liber de introductione loquendi di Filippino da Ferrara O.P.». Filologia mediolatina, 22, 319-67.

Godefroy, Frédéric (1881). Dictionnaire de l'ancienne langue française et de tous ses dialectes du IXe au XVe siècle. Paris: Vieweg. URL http:// micmap.org/dicfro/search/dictionnaire-godefroy (2017-10-25). 
Golden, Peter (1992). An Introduction to the History of Turkic Peoples. Wiesbaden: Harrassowitz. Turcologica 9.

Goldsmith, Kenneth (2017). Perdere tempo su Internet. Torino: Einaudi.

Gossen, Carl Theodor (1975). «Marco Polo und Rustichello da Pisa». Richthofen, Erich von; Bambeck, Manfred; Christmann, Helmut (Hrsgg.), Philologica Romanica Erhard Lommatzsch gewidmet. München: W. Fink, 133-43.

GRADIT = De Mauro, Tullio (a cura di) (1999-2000). Grande dizionario italiano dell'uso. 6 voll. Torino: UTET.

Grande, Stefano (1905). «Le relazioni geografiche fra P. Bembo, G. Fracastoro, G. B. Ramusio, G. Gastaldi». Memorie della Società Geografica Italiana, 12, 93-197.

Greenblatt,Stephen (1993). Renaissance Self-Fashioning. From More to Shakespeare. Chicago; London: University of Chicago Press.

Greenblatt, Stephen [1991] (1994). Meraviglia e possesso. Lo stupore di fronte al nuovo mondo. Bologna: il Mulino.

Grønbech, Kaare (1942). Komanisches Wörterbuch: türkischer Wortindex zu Codex Cumanicus. Kopenhagen: Munksgaard.

Guy, R. Kent (2010). Qing Governors and Their Provinces: The Evolution of Territorial Administration in China, 1644-1796. Seattle: University of Washington Press.

Hallberg, Ivar (1906). L'Extrême Orient dans la littérature et la cartographie de l'Occident des XIIIe, XIVe et XVe siècles. Göteborg: Zachrisson.

Harva, Uno (1938). Die religiösen Vorstellungen der altaischen Völker. Helsinki: Suomalainen Tiedeakatemia. FF Communications 125.

Haw, Stephen G. (2006). Marco Polo's China: A Venetian in the Realm of Khubilai Khan. New York: Routledge.

Huang, Xiaofeng 黄小峰 (2011). «Shiqiao, mufa yu 15 shiji de shangye kongjian: “Lugou yun fa tu” xin tan» 石桥、木筏与15世纪的商业空间: 《卢沟运 筏图》 新探 (Stone Bridge, Wood Rafts, and the Commercial Space in 15thcentury China: Re-approaching the Rafts at the Lugou Bridge). Zhongguo guojia bowuguan guankan 中国国家博物馆馆刊 (Journal of National Museum of China), 1, 66-79.

Ineichen, Gustav (a cura di) (1962-6). El libro Agregà de Serapiom, volgarizzamento di Frater Jacobus Philippus de Padua. Venezia; Roma: Istituto per la collaborazione culturale.

Italia, Paola (2016a). «Editing 2.0. Quali testi leggiamo e leggeremo in Rete?». Nuovi Argomenti, 73, 80-6.

Italia, Paola (2016b). «Pierazzo, Elena (2015). Digital Scholarly Editing: Theories, Models and Methods». Ecdotica, 13, 245-56.

Iwamura, Shinobu (1949). Manuscripts and Printed Editions of Marco Polo's "Travels". Tokyo: National Diet Library.

Janhunen, Juha (1986). Siberian Shamanistic Terminology. Lehtinen, Ildikó (ed.), Traces of the Central Asian Culture in the North. Helsinki: Suomalais- 
Ugrilainen Seura, 97-117. Suomalais-Ugrilaisen Seuran Toimituksia/ Mémoires de la Société Finno-Ougrienne 194.

Karrow, Robert W. (2007). «Centers of Map Publishing in Europe, 14721600». Harley, John Brian; Woodward, David (eds), The History of Cartography (1992-2007), vol. 3, pt. 1. Chicago: University of Chicago Press, 611-21.

Kiernan, Kevin (2006). «Digital Facsimiles in Editing». Burnard, Lou; O'Keeffe, Katherine O'Brien; Unsworth, John (eds), Electronic Textual Editing. New York: Modern Language Association of America, 262-8.

Kowalewski, Joseph É. (1844). Dictionnaire mongol-russe-français. Kazan': Imprimerie de l'Université.

Krader, Lawrence (1954). «Buriat Religion and Society». Southwestern Journal of anthropology, 10(3), 322-51.

Krader, Lawrence (1975). «The Shamanist Tradition of the Buryats (Siberia)». Anthropos, 70, 105-44.

Krader, Lawrence (1978). «Shamanism: theory and history in Buryat society». Dioszegi, Vilmos; Hoppal, Mihaly (eds), Bibliotheca Uralica, 1, 181-236.

Lefebvre, Henri [1975] (2000). La Production de l'espace. Paris: Anthropos. LEI = Pfister, Max; Schweickard, Wolfgang (1979-). Lessico Etimologico Italiano. Wiesbaden: Reichert.

Leonardi, Lino (2007). «Filologia elettronica tra conservazione e ricostruzione». Ciula, Arianna; Stella, Francesco (a cura di), Digital Philology and Medieval Texts. Ospedaletto (PI): Pacini, 65-75.

Leonardi, Lino (2017). «Le parole della tradizione filologica italiana: un modello da ripensare». Medioevo Romanzo, 41, 39-59.

Lessing, Ferdinand D. (1995). Mongolian-English Dictionary. 3a ed. Bloomington: The Mongolian society.

Lettere (1560). Lettere di XIII huomini illustri, nelle quali sono due libri di diuersi altri auttori, et il fiore di quante belle lettere, che sin'hora si sono uedute; con molte del Bembo, del Nauagero, del Fracastoro, del Manutio, $\&$ di altri famosi auttori non piu date in luce. Venezia: per Francesco Lorenzini da Turino.

Lot-Falck, Éveline (1956). «A propos d'Ätügän, déesse mongole de la terre». Revue de l'Historie des Religions, 149(2), 157-96.

Maggio, Silvia (2001). Il "Memoriale a se stesso" di Giovanni Carlo Scaramelli, diplomatico veneziano del secolo XVI [tesi di laurea]. Trieste: Università degli Studi di Trieste.

Manetti, Roberta (a cura di) (2000). «Rime di Antonio da Ferrara (Antonio Beccari) edite per il corpus testuale del Tesoro della Lingua Italiana delle Origini». Bollettino dell'Opera del vocabolario italiano, 5, 251-356.

Manžigeev, Ivan A. (1978). Burjatskie šamanističeskie i došamanističeskie terminy. Moskva: Nauka. 
Marchello-Nizia, Christiane; Lavrentiev, Alexey; Guillot-Barbance, Céline (2015). «Édition électronique de la Queste del saint Graal». Trotter, David (éd.), Manuel de la philologie de l'édition. Berlin; Boston: De Gruyter, 155-76.

Marchisio, Anna Lia (2016). Odorico da Pordenone: Relatio de mirabilibus orientalium Tatarorum. Firenze: SISMEL-Edizioni del Galluzzo.

Marsden, William (1818). The Travels of Marco Polo. London: printed by Cox and Baylis, Great Queen-Street, Lincoln's-Inn-Fields.

Marsden, William [1818] (1854). The Travels of Marco Polo, the Venetian. The Translation of Marsden Revised, With a Selection of His Notes. Ed. by Th. Wright. London: H.G. Bohn.

Martin, Samuel E. (1961). Dagur Mongolian Grammar, Text and Lexicon. Bloomington: Indiana University. Indiana University Uralic and Altaic series 4.

Mascherpa, Giuseppe (2007-08). Nuove indagini sulla tradizione latina Z del "Milione" di Marco Polo [tesi di dottorato]. Siena: Università degli Studi.

Mascherpa, Giuseppe (2008). «San Tommaso in India. L'apporto della tradizione indiretta alla costituzione dello stemma del Milione». Cadioli, Alberto; Chiesa, Paolo (a cura di), Prassi ecdotiche. Esperienze editoriali su testi manoscritti e testi a stampa (Milano, 7 giugno e 31 ottobre 2007). Milano: Cisalpino, 171-84.

Mascherpa, Giuseppe (2011). «Il primo libro». Burgio 2011, 45-77.

Mascherpa, Giuseppe (in corso di stampa). «Una Venezia d’Oriente. Gli splendori di Quinsai nella tradizione del Devisement dou monde». Mascherpa, Giuseppe; Strinna, Giovanni (a cura di), Predicatori, mercanti, pellegrini. L'Occidente medievale e lo sguardo letterario sull'Altro tra l'Europa e il Levante. Mantova: Universitas Studiorum.

Membré, Michele [1542] (1969). Relazione di Persia (1542). Napoli: Istituto Universitario Orientale.

Ménard, Philippe (2001-09). Marco Polo: "Le devisement du monde", publ. sous la dir. de Philippe Ménard. 6 voll. Genève: Droz. Textes littéraires français 533, 552, 568, 575, 586, 597.

Ménard, Philippe (2005). «L'édition du Devisement du Monde de Marco Polo». Comptes rendus des séances de l'Académie des Inscriptions et Belles-Lettres, 1, 407-35.

Ménard, Philippe (2008). «Marco Polo et la mer. Le retour de Marco Polo en Occident d'après les diverses versions du texte». Conte 2008, 173-204.

Ménard, Philippe (2010). «Marco Polo et la mer: les navires vus en Orient». Le passions d'un historien: mélanges en l'honneur de Jean-Pierre Poussou. Paris: Presses de l'université Paris-Sorbonne, 415-24.

Ménard, Philippe (2012). «Deux nouveaux folios inédits d'un fragment franco-italien du Devisement du monde de Marco Polo». Medioevo Romanzo, 36, 241-80. 
Ménard, Philippe (2017). «Marco Polo transposé en latin par Pipino». Goudeau, Emilie; Laurent, Françoise; Quereuil, Michel (éds.), «Le monde entour et environ». La geste, la route et le livre dans la littérature médiévale. Mélanges offerts à Claude Roussel. Clermont-Ferrand: Presses universitaires de l'université Blaise Pascal, 193-205.

Mencke, Otto (1731). De vita, moribus, scriptis, meritisque in omne literarum genus prorsus singularibus Hieronymi Fracastorii Veronensis. Lipsia: Breitkopf.

Mignolo, Walter (1995). The Darker Side of the Renaissance. Literacy, Territoriality, and Colonization. Ann Arbor: The University of Michigan Press.

Mignolo, Walter (2011). «The Darker Side of the Enlightenment: A DeColonial Reading of Kant's Geography». Elden, Stuart; Mendieta, Eduardo (eds), Reading Kant's Geography. Albany: State University of New York Press, 319-43.

Milanesi, Marica (a cura di) (1978-88). Giovanni Battista Ramusio. Navigazioni e viaggi. 6 voll. Torino: Einaudi.

Milanesi, Marica (1984). Tolomeo sostituito. Studi di storia delle conoscenze geografiche nel XVI secolo. Milano: Unicopli.

Milanesi, Marica (1994). «Giovanni Battista Ramusio e le Navigazioni e viaggi (1550-1559)». Zorzi, Renzo (a cura di), L'epopea delle scoperte. Firenze: Leo S. Olschki Editore, 75-101.

Minervini, Laura (2012). «Les emprunts arabes et grecs dans le lexique français d'Orient (XIIIe-XIVe siècles)». Revue de linguistique romane, 76, 99-198.

Morgan, David (1997). Breve storia dei Mongoli. Trad. di Barbara Massari. Milano: Mondadori. Trad. di: The Mongols, Oxford: Basil Blackwell, 1987. Mostaert, Antoine (1957). «Le mot Natigay / Nacigay chez Marco Polo». Balasz, Étienne (a cura di), Oriente Poliano. Studi e conferenze tenute all'Is.M.E.O. in occasione del VII centenario della nascita di Marco Polo (1254-1954). Roma: Istituto Italiano per il Medio ed Estremo Oriente.

Mostaert, Antoine (2009). Dictionnaire Ordos. 3a ed. Ulaanbaatar: New Polygraph.

Motta, Attilio; Robins, William (a cura di) (2007). Antonio Pucci. Cantari della Reina d'Oriente. Bologna: Commissione per i testi di lingua.

Moule, Arthur C. (1925-26). «Carriages in Marco Polo's Quinsai». T'oung Pao, 24(1), 66-9.

Moule, Arthur C. (1957). Quinsai. With other notes on Marco Polo. Cambridge: University Press.

Moule, Christopher Arthur; Pelliot, Paul (1938). Marco Polo: The Description of the World. 2 vols. London: Routledge.

Nocentini, Alberto (1993). «Aretino sirca e termini correlati». Lingua Nostra, 54, 1-3. 
Palladius, Archimandrite (1876). «Elucidations of Marco Polo's Travels in North-China, drawn from Chinese sources». Journal of the China Branch of the Royal Asiatic Society, n.s., 10, 1-54.

Pallas, Peter Simon (1794). Voyages du professeur Pallas, dans plusieurs provinces de l'empire de Russie et dans l'Asie septentrionale. Paris: Chez Maradan.

Paris, Gaston; Pannier, Leopold (1872). La vie de saint Alexis: poème du 11. siècle et renouvellements des 12., 13. et 14. siécles. Publiés avec préfaces, variantes, notes et glossaire. Paris: Librairie A. Franck.

Parks, George B. (1955a). «The Contents and Sources of Ramusio's Navigationi». Bulletin of the New York Library, 59(6), 279-313.

Parks, George B. (1955b). «Ramusio's Literary History». Studies in Philology, 52, 127-48.

Pauthier, Guillaume (éd.) (1865). Le livre de Marco Polo, citoyen de Venise, conseiller privé et commissaire imperial de Khoubilaï-Khaân, rédigé en français sous sa dictée en 1298 par Rusticien de Pise. 2 voll. Paris: Didot.

Pelliot, Paul (1959-73). Notes on Marco Polo. Ouvrage posthume, publié sous les auspices de l'Académie des Inscriptions et Belles-Lettres et avec le concours du Centre national de La Recherche scientifique. 3 voll. Paris: Imprimerie nationale.

Peretti, Aurelio (1930). «Per la storia del Testo di Marco Polo». Archivio storico italiano, s. 7, 13(88), 217-47.

Pierazzo, Elena (2011). «A Rationale of Digital Documentary Editions». Literary and Linguistic Computing, 26, 463-77.

Pierazzo, Elena (2016). «Modelling Digital Scholarly Editing: From Plato to Heraclitus». Driscoll; Pierazzo 2016a, 41-58.

Poncelet, Albert (1910). «Le légendier de Pierre Calo». Analecta Bollandiana, 29, 1-131.

Prášek, Justin V. (1902). M. Pavlova z Benátek: Milion. v Praze: Ées. Akademie. Price, Kenneth M. (2009). «Edition, Project, Database, Archive, Thematic Research Collection: What's in a Name?».Digital Humanities Quarterly, 3(3). URL http://www.digitalhumanities.org/dhq/vol/3/3/000053/000053. html (2017-11- 09).

Purev, Otgony; Purvee, Gurbadaryn (2010). Mongolian shamanism. Revised and updated fifth edition, volumes 1 and 2. Ulaanbaatar: Mönxijn üseg. Quijano, Aníbal (2010). «Coloniality and Modernity/Rationality». Mignolo, Walter; Escobar, Arturo (eds), Globalization and the Decolonial Option. London; New York: Routledge, 22-32.

Radloff, Wilhelm (1893). Aus Siberien. Loese Blaetter aus meinem Tagebuche. 2 Bde. Zweite Ausgabe. Leipzig: T.O. Weigel.

Ragagnin, Elisabetta (2011). Dukhan, a Turkic variety of northern Mongolia: Description and Analysis. Wiesbaden: Harrassowitz. Turcologica 76.

Ramstedt, Gustaf J. (1935). Kalmückisches Wörterbuch. Helsinki: SuomalaisUgrilainen Seura. 
Ramusio, Giovanni Battista (1550). Primo volume delle navigationi et viaggi. Venezia: Giunti.

Ramusio, Giovanni Battista (1556). Terzo volume delle navigationi et viaggi. Venezia: Giunti.

Ramusio, Giovanni Battista (1559). Secondo volume delle navigationi et viaggi. Venezia: Giunti.

Ramusio, Giovanni Battista (1563). Primo volume, et terza edizione delle navigationi et viaggi raccolto già da $\mathrm{m}$. Gio. Battista Ramusio. Venezia: Giunti.

Ratchnevsky, Paul (1970). «Über den mongolischen Kult am Hofe der Grosskhane in China». Ligeti, Louis (Hrsg.), Mongolian Studies. Budapest: Akadémiai Kiadó, 417-43.

Reginato, Irene (2015-16). La version $K$ (catalane) $d u$ "Devisement $d u$ monde"/"Milione" de Marco Polo: Recherches et éditions [thèse de doctorat]. Venise; Paris: Università Ca' Foscari Venezia; École Pratique des Hautes Études.

Ribaudo, Vera (2013). «Nuovi orizzonti dell'ecdotica? L'edizione elettronica della Monarchia e della Commedia di Prue Shaw». L'Alighieri, 42, 95-127.

Rieger, Dietmar (1992). «Marco Polo und Rustichello da Pisa. Der Reisende und sein Erzähler». Ertzdorff, Xenia von; Neukirch, Dieter (Hrsgg.), Reisen und Reiseliteratur im Mittelalter und in der Frühen Neuzeit. Unter redaktioneller Mitarbeit von Rudolf Schulz. Amsterdam; Atlanta: Rodopi, 289-312.

Rinaldin, Anna (in corso di stampa). Glossario dei realia notevoli in $R$. Simion, Burgio 2015.

Robinson, Peter (2005). «Current Issues in Making Digital Editions of Medieval Texts - or, Do Electronic Scholarly Editions Have a Future?». Digital Medievalist, 1. URL https://journal.digitalmedievalist.org/ articles/10.16995/dm.8/print/ (2018-01-06).

Robinson, Peter (2016). «The Digital Revolution in Scholarly Editing». Crostini, Barbara; Iversen, Gunilla; Jensen, Brian M. (eds), Ars Edendi Lecture Series IV. Stockholm: Stockholm University Press, 181-207.

Rockhill, William W. (1900). The Journey of William of Rubruck to the Eastern Parts, 1253-55 as Narrated by Himself, with Two Accounts of the Earlier Journey of John of Pian de Carpine. Translated, and edited, with an Introductory Notice by W.W. Rockhill. London: Hakluyt Society.

Romanini, Fabio (2007). 'Se fussero più ordinate, e meglio scritte...'. Giovanni Battista Ramusio correttore ed editore delle "Navigationi et viaggi". Roma: Viella.

Romanini, Fabio (2013). «Nota sul testo della Cosmographia et Geographia de Affrica di Giovanni Leone Africano». Arcodia, Giorgio Francesco; Da Milano, Federica; Iannaccaro, Gabriele; Zublena, Paolo (a cura di), Tilelli. Scritti in onore di Vermondo Brugnatelli. Roma: Caissa Italia Editore, 153-63. 
Roncaglia, Gino (2010). La quarta rivoluzione. Roma-Bari: Laterza.

Roux de Rochelle, Jean-Baptiste Gaspard (1990). La religione dei turchi e dei mongoli. Gli archetipi del naturale negli ultimi sciamani. Genova: ECIG.

Roux de Rochelle, Jean-Baptiste Gaspard (1824). Voyages de Marco Polo. Paris: Société de Géographie.

Sahle, Patrick (2016). «What is a Scholarly Digital Edition?». Driscoll, Pierazzo 2016a, 19-39. URL https://wWw.openbookpublishers.com/product/483 (2017-11-09).

Sahle, Patrick (2017). A Catalog of Digital Scholarly Editions. URL http:// www.digitale-edition.de/ (2017-11-09).

Sansovino, Francesco (1581). Venetia città nobilissima et singolare. Venezia: Giacomo Sansovino.

Sanudo, Marin (1899) (a cura di). «Summario delle cose de David judeo, fiol del re Salamon de Tabor et fratello del re Joseph venuto novamente in Venetia». I Diarii, vol. 54. Venezia: Visentini, coll. 145-8.

Schafer, Edward H. (1956). «The Development of Bathing Customs in Ancient and Medieval China and the History of the Floriate Clear Palace». Journal of the American Oriental Society, 76(2), 57-82.

Segre, Cesare (2008). «Chi ha scritto il Milione di Marco Polo?». Conte 2008, 5-16.

Segre, Cesare; Marti, Mario (a cura di) (1959). La prosa del Duecento. Milano; Napoli: Ricciardi.

Sella, Pietro (1944). Glossario latino-italiano: Stato della Chiesa, Veneto, Abruzzi. Città del Vaticano: Biblioteca Apostolica Vaticana.

Shaw, Prue (ed.) (2006). Dante Alighieri: "Monarchia". A Digital Edition [DVD]. Birmingham: Scholarly Digital Edition.

Shaw, Prue (ed.) (2010). Dante Alighieri: "Commedia". A Digital Edition [DVD]. Birmingham; Firenze: Scholarly Digital Edition; SISMEL-Edizioni del Galluzzo.

Shillinburg, Peter (1996). Scholarly Editing in the Computer Age: Theory and Practice. Ann Arbour (MI): University of Michigan Press.

Shimamura, Ippei (2014). The Roots Seekers: Shamanism and Ethnicity among the Mongol Buryats. Yokohama: Shumpusha Publishing.

Short, John R. (2004). Making Space: Revisioning the World. Syracuse (NY): Syracuse University Press.

Simion, Samuela (2008-09). Il "Milione" secondo il manoscritto Hamilton 424 della Staatsbibliothek di Berlino. Edizione critica [tesi di dottorato]. Venezia: Università Ca' Foscari.

Simion, Samuela (2011). Struttura e fonti di V. Burgio 2011, 27-44.

Simion, Sanuela (2015a). La redazione P. Simion, Burgio 2015. URL http:// virgo.unive.it/ecf-workflow/books/Ramusio/testi_completi/P marcato-main.html (2018-09-28). 
Simion, Samuela (2015b). La redazione R. Simion, Burgio 2015. URL http:// virgo.unive.it/ecf-workflow/books/Ramusio/testi completi/R marcato-main.html (2018-09-28).

Simion, Samuela (2015c). La redazione V. Simion, Burgio 2015. URL http:// virgo.unive.it/ecf-workflow/books/Ramusio/testi completi/V marcato-main.html (2018-09-28).

Simion, Samuela (a cura di) (2016). Benedetto, Luigi Foscolo: Livre de messire Marco Polo citoyen de Venise, appelé Milion, où sont décrites les Merveilles du monde. Traduzione critica secondo le carte inedite del lascito di Ernest Giddey. Venezia: Edizioni Ca' Foscari. DOI 10.14277/97888-6969-103-4 (2017-11-09).

Simion, Samuela (2017a). «La vita di Buddha nel Milione veneziano V». Divizia, Paolo; Pericoli, Lisa (a cura di), Il viaggio del testo $=$ Atti del Convegno internazionale di Filologia italiana e romanza (Brno, 19-21 giugno 2014). Alessandria: Edizioni dell'Orso, 19-21.

Simion, Samuela (2017b). «Online-Ressourcen. Giovanni Battista Ramusio, Dei Viaggi di Messer Marco Polo, Edizione critica digitale». Zeitschrift für romanische Philologie, 133(4), 1257-67.

Simion, Samuela (in corso di stampa (a)). «Orienti vecchi e nuovi nel Lapidario del Prete Gianni». Mascherpa, Giuseppe; Strinna, Giovanni (a cura di), Predicatori, mercanti, pellegrini. L'Occidente medievale e lo sguardo letterario sull'Altro tra l'Europa e il Levante = Atti del Convegno Internazionale di Studi (Sassari, 11-12 maggio 2016). Mantova: Universitas Studiorum.

Simion, Samuela; Burgio, Eugenio (2015). Ramusio, Giovanni Battista: Dei viaggi di Messer Marco Polo. Edizione critica digitale progettata e coordinata da Eugenio Burgio, Marina Buzzoni, Antonella Ghersetti. Venezia: Edizioni Ca' Foscari. URL http://virgo.unive.it/ecf-workflow/ books/Ramusio/main/index.html (2018-09-28).

Skribnik, Elena (2003). «Buryat». Janhunen, Juha (ed.), The Mongolic Languages. London; New York: Routledge, 102-28.

Stachowski, Marek (1993). Dolganischer Wortschatz. Kraków: Uniwersytet Jagielloński.

Stussi, Alfredo (1994). Introduzione agli studi di filologia italiana. Bologna: il Mulino.

Tatár, Magdolna (2003). The First Tatars in Europe. Sárközi, Alice; Rákos, Attila (eds), Altaica Budapestinensia = Proceedings of the 45th Permanent International Altaistic Conference (PIAC) (Budapest, Hungary, 23-25 June 2002). Budapest: Hungarian Academy of Sciences, Dept. of Inner Asian Studies; Eötvös Loránd University, 328-51.

Terracini, Benvenuto (1933). «Ricerche ed appunti sulla più antica redazione del Milione». Rendiconti della Reale Accademia Nazionale dei Lincei, s. 6, 9, 369-428. 
Terracini, Benvenuto [1957] (1983). Il problema della traduzione. A cura di Mortara Garavelli, Bice. Milano: Serra e Riva. Or. ed.: Conflitti di lingua e di cultura. Venezia: Neri Pozza, 1957, 49-121.

Thackston, Wheeler McIntosh (ed.) (1998-99). Rashiduddin Fazlullah's Jami u t-tawarikh: Compendium of Chronicles. A History of the Mongols. English translation ad annotation by W.M.T. 3 vols. Cambridge (MA): Harvard University, Department of Near Eastern Languages and Civilizations. Sources of Oriental languages and literatures 45; Sources of Oriental languages and literatures: Central Asian sources 4.

Tomasin, Lorenzo (2001). Il volgare e la legge. Storia linguistica del diritto veneziano (secoli XIII-XVIII). Padova: Esedra.

Tommaseo, Nicolò; Bellini, Bernardo (1861-79). Dizionario della lingua italiana. Torino: Unione tipografico-editrice.

Transilvano, Massimiliano; Pigafetta, Antonio (1536). Il viaggio fatto da gli Spagniuoli a torno a 'l mondo. Venezia: [Giunti].

Travi, Ernesto (a cura di) (1987-93). Pietro Bembo: Lettere. 4 voll. Bologna: Si cita per volume e numero della lettera.

Trebbi, Giuseppe (1986). «Il segretario veneziano. Una descrizione cinquecentesca della cancelleria ducale». Archivio Storico Italiano, 144, 35-73.

Trovato, Paolo (2009). «Di alcune edizioni recenti di Antonio Pucci, del codice Kirkup e della cladistica applicata alla critica testuale». Bettarini Bruni, Trovato 2009, 81-97.

Tucci, Giuseppe; Heissig, Walther (1970). Die Religionen Tibets und der Mongolei. Stuttgart; Berlin; Köln; Mainz: Kohlhammer.

Vanderdorpe, Christian (1999). Du papyrus à l'hypertexte. Essai sur les mutations du texte et de la lecture. Montréal; Paris: Boréale; La Découverte. URL http://litmedmod.ca/sites/default/files/pdf/vandendorpe-papyrusenligne_lr.pdf (2018-01-03).

Varvaro, Alberto [1970] (2004). «Critica di testi classica e romanza. Problemi comuni ed esperienze diverse». Varvaro, Alberto, Identità linguistiche $e$ letterarie nell'Europa romanza. Roma: Salerno Editrice, 567-612.

Veneri, Toni (2012a). «Giovanni Battista Ramusio, molto più di uno spettatore. Le quinte delle Navigationi et viaggi». Italica, 89(2), 162-201.

Veneri, Toni (2012b). «Il riscatto geografico di Marco Polo». Quaderni Veneti, 1(2), 33-57. DOI 10.14277/1724-188X/QV-1-2-12-3.

Ventura, Angelo (1980). «Scrittori politici e scrittori di governo». Storia della cultura veneta. Dal primo Quattrocento al Concilio di Trento, 3(3). Vicenza: Neri Pozza, 513-63.

Verbickij, V. [1884] (2005). Slovar' Altajskogo i Aladagskogo narečij tjurkskogo jazyka. Gorno-Altajsk: Ak Čeček.

Veyne, Paul (1971). Comment on écrit l'histoire: essai d'épistémologie. Paris: Seuil. 
Vidos, Benedek E. (1939). Storia delle parole marinaresche italiane passate in francese: contributo storico linguistico all'espansione della lingua nautica italiana. Firenze: Leo S. Olschki Editore.

Voltolina, Piero (1998). La storia di Venezia attraverso le medaglie. 3 voll. Venezia: P. Voltolina.

Weinreich, Uriel (1974). Lingue in contatto. Trad. di Giorgio Raimondo Cardona. Torino: Bollati Borlinghieri. Trad. di: Languages in Contact: Findings and Problems. New York: Linguistic Circle of New York, 1953.

White, Hayden (1973). Metahistory: The Historical Imagination in NineteenthCentury. Baltimore: Johns Hopkins University Press.

White, Hayden (1999). «Auerbach's Literary Theory. Figural Causation and Modernist Historicism». Figural Realism: Studies in the Mimesis Effect. Baltimore; London: Johns Hopkins University Press, 87-100.

Woodward, David (2002). Cartografia a stampa nell'Italia del Rinascimento. Trad. di Francesca Albini. Milano: Sylvestre Bonnard.

Yule, Henry; Cordier, Henri (eds) [1871] (1903). The Book of Ser Marco Polo, the Venetian, Concerning the Kingdoms and Marvels of the East. 2 vols. Third edition revised in the light of recent discoveries by Henri Cordier London: John Murray.

Žamcarano, Cyben Ž. (1909). «Ongoty aginskix burjat». Sbornik v čest' 70-letija Grigorija Nikolaeviča Potanina. St. Petersburg: Tip. V. F. Kiršbauma, 379-94.

Zannini, Andrea (1993). Burocrazia e burocrati a Venezia in età moderna: $i$ cittadini originari (XVI-XVIII). Venezia: Istituto Veneto di Scienze, Lettere ed Arti.

Zelenin, Dmitrij K. (1936). Kult ongonov v Sibiri. Perežitki totemizma v ideologii sibirskix narodov. Moskva: Izdvo Akademiinauk.

Zenker, Julius Theodor (1866-76). Türkisch-arabisch-persisches Handwörterbuch. Dictionnaire Turc-Arabe-Persan. 2 Bde. Leipzig: von Wilhelm Engelmann.

Zinelli, Fabio (2016). «Espaces franco-italiens: les italianismes du françaismédiéval». Glessgen, Martin; Trotter, David A. (éds.), La régionalité lexicale du français au Moyen Âge: volume thématique issu du colloque de Zurich, 7-8 sept. 2015. Strasbourg: ÉliPhi, 207-69.

Zurla, Placido (1806). Il "Mappamondo" di Fra Mauro camaldolese descritto e illustrato da d. P.Z. dello stess'Ordine. Venezia: s.n. 



\section{Rivista semestrale}

Dipartimento di Studi Umanistici Università Ca' Foscari Venezia 